\title{
Theoretical Studies of the Dynamics of Action Potential Initiation and its Role in Neuronal Encoding
}

\author{
Dissertation
}

zur Erlangung des mathematisch-naturwissenschaftlichen

Doktorgrades

"Doctor rerum naturalium"

der Georg-August-Universität Göttingen

vorgelegt von

Wei Wei

aus Liaoning, P. R. China

2010 
First Referee / Advisor:

Second Referee:

Committee members:
Prof. Dr. Fred Wolf

Prof. Dr. Kree Reiner

Prof. Dr. Tobias Moser

Prof. Dr. Eberhard Bodenschatz

Date of submission of the PhD thesis: November 30th, 2010 Date of thesis defence:

January 21th, 2011 
Herewith I declare, that I prepared the $\mathrm{PhD}$ Thesis

"Theoretical Studies of the Dynamics of Action Potential Initiation and its Role in Neuronal Encoding"

on my own and with no other sources and aids than quoted.

Göttingen, 2010

Wei Wei 


\section{Contents}

Contents 3

\begin{tabular}{ll}
\hline List of Figures & 7
\end{tabular}

1 Introduction 1

$\begin{array}{lll}2 & \text { Spike onset dynamics and population response } & 7\end{array}$

2.1 An analvticallv solvable model with an unstable fixed point. . . . 7

2.2 Stationarv solutions . . . . . . . . . . . . . . . . . 10

2.3 Linear responses and relation to the LIF model . . . . . . . . . . 14

2.3 .1 Linear response to a current coded signal . . . . . . . . . . 14

2.3 .2 Linear response to a noise coded signal . . . . . . . . . . . 18

2.3 .3 Evaluation of the results . . . . . . . . . . . . . . . 20

2.3 .4 Relation to the LIF model . . . . . . . . . . . . . . . . . 22

2.3 .5 Step response . . . . . . . . . . . . . . . . . . . . . . 23

2.4 Large $v_{b}$ limit and separation of boundary induced artifacts . . . . 24

$2.4 .1 \quad$ Large $v_{b}$ limit . . . . . . . . . . . . . . . . . . . 24

2.4 .2 Threshold models and boundary induced artifacts . . . . . 28

2.4 .3 Separation of boundary induced artifacts . . . . . . . . . 28

2.5 Low rate limit . . . . . . . . . . . . . . . . . . . . . . . . . . . . 29

2.6 High frequencv behavior: $r$ and $f_{0} \ldots \ldots \ldots \ldots . \ldots . \ldots 29$

$2.7 \quad r=0$ case: a new kind of perfect integrator $\ldots \ldots \ldots \ldots$

2.8 Discussion . . . . . . . . . . . . . . . . . . . . . . . . . . 37

2.9 Appendix: parabolic cylinder functions . . . . . . . . . . . 39

3 Toward high cutoff frequencies for current coded signals 43

3.1 Step change in sodium influx and its modeling . . . . . . . . . . 45

3.2 Stationary solutions . . . . . . . . . . . . . . . . . . . 47

3.3 Linear responses: directly solving the FPE . . . . . . . . . . . 50

3.3 .1 Linear response to a current coded signal . . . . . . . . . 50

3.3.2 Linear response to a noise coded signal ... . . . . . . . . 52

3.4 Large $v_{b}$ limit and separation of boundary induced artifacts . . . . $\quad 55$

3.5 Linear response: limit behavior $\ldots \ldots \ldots \ldots \ldots$ 
3.5.1 Large $r$ limit and large $\omega$ limit . . . . . . . . . . . . . 57

$3.5 .2 \quad$ Large $\Delta \dot{v}$ limit . . . . . . . . . . . . . . . . 57

3.5.3 High frequency behavior: effect of the step change with white noise . . . . . . . . . . . . . . 58

3.6 Effect of correlation time $\tau_{s} \ldots \ldots \ldots \ldots \ldots \ldots \ldots$

3.6 .1 Modeling correlated svnaptic noise . . . . . . . . . . . 58

3.6 .2 Stationarv firing rate $\nu_{0} \ldots \ldots \ldots \ldots$. . . . . . . . . . . 59

3.6 .3 Interplav of $\tau_{s}$ and $r$ without a step change . . . . . . . 59

3.6 .4 Interplay of $\tau_{s}$ and $\Delta i \quad \ldots \ldots \ldots \ldots \ldots$

3.7 Discussion . . . . . . . . . . . . . . . . . . . 62

4 A general piecewise linear model 65

4.1 Description of the model . . . . . . . . . . . . . . . 65

4.2 Stationarv solutions . . . . . . . . . . . . . . . . . . . . . 68

4.3 Linear responses . . . . . . . . . . . . . . . . . . . . . . . . . . . . . $\quad 69$

4.3.1 Linear response to a current coded signal . . . . . . . . . 69

4.3 .2 Linear response to a noise coded signal . . . . . . . . . . . 73

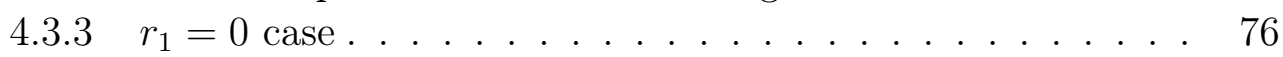

4.4 Limit behaviors . . . . . . . . . . . . . . . . . . . . . 77

$4.4 .1 \quad$ Large $\omega$ limit . . . . . . . . . . . . . . . . . . 77

4.4.2 $v_{1} \rightarrow v_{0}$ limit: relation to the previous models . . . . . 77

4.4 .3 Large $v_{b}$ limit . . . . . . . . . . . . . . . . . 79

4.5 Influence of details of AP generation on the dynamical response . $\quad 80$

4.5.1 Influence of sub-threshold dvnamics . . . . . . . . . 80

4.5.2 Influence of spike initiation dvnamics . . . . . . . . . . 81

4.6 Fitting the EIF model with a 3-piece model . . . . . . . . . 86

4.7 Signal encoding through barrier penetration in a bistable system . 89

4.8 Discussion: membrane dynamics and dynamical response . . . . . 91

4.9 Appendix $\ldots \ldots \ldots \ldots \ldots$

5 Dynamical AP encoding in spatially extended neurons 101

5.1 The cable equation . . . . . . . . . . . . . . . . . . . 102

5.2 Membrane potential as a Gaussian random field . . . . . . . . . . 104

5.3 Transfer function for a homogeneous cable . . . . . . . . . . . . 106

5.3 .1 Simple ball-and-stick model . . . . . . . . . . . . . . . 106

5.3 .2 Two-compartment cable . . . . . . . . . . . . . . . . . 108

5.4 Spike-triggered average . . . . . . . . . . . . . . . . . . . . . . 109

5.5 Linear response . . . . . . . . . . . . . . . . . . . . . . . 113

5.5 .1 Two coupled Gaussian neurons . . . . . . . . . . . . . 113

5.5 .2 Two-compartment cable . . . . . . . . . . . . . . . 113

5.5.3 Relation between spike-triggered average and linear response 116

5.6 Discussion . . . . . . . . . . . . . . . . . . . . . . . . . 116

$5.7 \quad$ Appendix $\ldots \ldots \ldots \ldots \ldots$ 
6 Summary and Perspective 125

Bibliography

Acknowledgements

Curriculum Vitae 


\section{List of Figures}

2.1 A. illustration of the model. B. $V(t)$ trajectories for identical noise and three different values of $\mathrm{r}$ in the noise driven regime. . . . . .

2.2 Dependence of the stationary probability density on the membrane potential with firing rate $5 \mathrm{~Hz}(\mathrm{~A})$ and $10 \mathrm{~Hz}(\mathrm{~B})$. Parameters used here and in other figures in this chapter: $\tau_{m}=10 \mathrm{~ms}, \mu=0$, $v_{r}=0, \tau_{r}=0$ and $v_{b}=10$ if not denoted otherwise. . . . . . .

2.3 The dependence of stationarv firing rate on the mean input current (A) and the noise strength (B) in the noise driven regime. . . . .

2.4 The dependence of stationary firing rate $\nu_{0}$ on the boundary $v_{b}$ for different $r$ with $\mu=0 . \ldots \ldots \ldots$

2.5 Comparison of the stationary firing rate $\nu_{0}$ with its approximation Eq. (2.18) (labeled as 'app') and the Kramers rate for different $r$.

2.6 The normalized transmission functions $\nu_{1}(\omega) / \nu_{1}(0.1)$ and phase lags for a current coded signal $(\mathrm{A}, \mathrm{B})$ and for a noise coded signal (C,D) with $r=1$ and $\nu_{0}=5 \mathrm{~Hz}$. The dashed and solid lines are theoretical results and the points are from numerical simulation using Eq. (2.53) and (2.54). . . . . . . . . . . . . .

2.7 The normalized transmission functions $\nu_{1}(\omega) / \nu_{1}(0.1)$ and phase lags for a current coded signal (A,B) and for a noise coded signal $(\mathrm{C}, \mathrm{D})$ with $r=10$ and $\nu_{0}=5 \mathrm{~Hz} . \ldots \ldots \ldots$

2.8 The normalized transmission functions $\nu_{1}(\omega) / \nu_{1}(0.1)$ and phase lags for the current coded signal (A,B) and for the noise coded $\operatorname{signal}(\mathrm{C}, \mathrm{D})$ with $r=100$ and $\nu_{0}=5 \mathrm{~Hz} \ldots \ldots \ldots$ The instantaneous firing rate for a step change in the mean input(upper panel) and noise amplitude(lower panel) at $t=0$ for $r=10,100$. $\nu(t)$ changes from $5 \mathrm{~Hz}$ to $7 \mathrm{~Hz}$ in both cases. The solid lines are theoretical results from the linear response, Eq. (2.56). Note that the contributions from $\nu_{1}^{H i g h}(\omega)$ are negligible. The differences between simulation and theory are from higher order contributions. . . . . . . . . . . . . .

2.10 Dependence of the linear response on the truncation point $v_{b}$ for a current coded signal (A,B) and for a noise coded signal (C,D) with $r=10$ and $\nu_{0}=5 \mathrm{~Hz} \ldots \ldots \ldots \ldots$. . . . . . . . 
2.11 Dependence of the linear response on the truncation point $v_{b}$ for a current coded signal (A,B) and for a noise coded signal (C,D) with $r=100$ and $\nu_{0}=5 \mathrm{~Hz}$. . . . . . . . . . . . . .

2.12 The low rate approximation for the linear response with $r=$ 10, 100 and $\nu_{0}=0.01,0.1$ and $1 \mathrm{~Hz}$. A and $\mathrm{C}$ are for a current coded signal; B and D are for a noise coded signal. Solid lines are the accurate results from Eq. (2.40) and (2.51); dashed lines are the low rate approximations from Eq. (2.64). . . . . . . .

2.13 The asymptotic approximation Eq. (2.65) for $\nu_{1}^{\text {Low }}(\omega)(\mathrm{A}, \mathrm{C})$ and Eq. 2.62) for $\nu_{1}^{\text {High }}(\omega)$ (B, D) in the linear response with $r=$ 1, 10.100 and $\nu_{0}=5 \mathrm{~Hz}$. For the $\nu_{1}^{\text {Low }}(\omega)$ parts we normalize with the theoretical values at $f=0.1 \mathrm{~Hz} . \ldots \ldots$. . . . .

2.14 Left, the normalized transmission function $\nu_{1}^{\text {Low }}(\omega) / \nu_{1}(0.1)$ for a current coded signal (A) and for a noise coded signal (C) with different r. $\nu_{0}=5 \mathrm{~Hz}$. Right, the variation of the cut-off frequency (B for a current coded signal and D for a noise coded signal) with the onset rapidness $r$ for different firing rates: $\nu_{0}=$ $1,5,10,20,30,40 \mathrm{~Hz}$ from lower to upper curves.

3.1 Illustration of the model. .

3.2 Dependence of the stationary probability density on the membrane potential for $\Delta \dot{v}=0.5(\mathrm{~A}, \mathrm{~B})$ and $\Delta \dot{v}=2(\mathrm{C}, \mathrm{D})$. Parameters used here and in other figures in this chapter: $\tau_{m}=10 \mathrm{~ms}, \mu=0$, $v_{r}=0, \tau_{r}=0$ and $v_{b}=10$ if not denoted otherwise. . . . . . .

3.3 Dependence of the stationary firing rate on the noise strengh $\sigma$ for $\Delta \dot{v}=0(\mathrm{~A}), \Delta \dot{v}=0.5(\mathrm{~B}), \Delta \dot{v}=1(\mathrm{C})$ and $\Delta \dot{v}=5(\mathrm{D})$. Dashed lines are approximation from Eq. (3.16) and Eq. (3.17). . . . . .

3.4 The normalized transmission functions $\nu_{1}(\omega) / \nu_{1}(0.1)$ and phase lags for a current coded signal $(\mathrm{A}, \mathrm{B})$ and for a noise coded signal (C,D) with $r=1, \Delta \dot{v}=0.5$ and $\nu_{0}=5 \mathrm{~Hz}$. The dashed and solid lines are theoretical results and the points are from numerical simulation using Eq. (2.53) and (2.54). . . . . . . . . . .

3.5 The normalized transmission functions $\nu_{1}(\omega) / \nu_{1}(0.1)$ and phase lags for a current coded signal $(\mathrm{A}, \mathrm{B})$ and for a noise coded signal (C,D) with $r=10, \Delta \dot{v}=0.5$ and $\nu_{0}=5 \mathrm{~Hz}$. . . . . . . . .

3.6 The normalized transmission functions $\nu_{1}(\omega) / \nu_{1}(0.1)$ with step change $\Delta \dot{v}=1,5,10$ and for the corresponding LIF model results.

3.7 The dependence of stationary firing rate $\nu_{0}$ on the correlation time $\tau_{s}$ with different step change $\Delta \psi$. From A to D the step change are : $\mathrm{A}, \Delta \dot{v}=0, \mathrm{~B}, \Delta \dot{v}=0.5, \mathrm{C}, \Delta \dot{v}=1, \mathrm{D}, \Delta \dot{v}=5$. In each figures the curves from upper to lower are for $\tau_{s}=1,5,10,20 \mathrm{~ms}$ respectively. $r$ is fixed to be $10 \ldots \ldots \ldots$. . . . . . . 
3.8 The dependence of the the transmission function $\left|\nu_{1 c}(\omega) / \nu_{1 c}(0.1)\right|$ on the boundary $v_{b}$ for $\tau_{s}=1,10 \mathrm{~ms}$ and $r=1,10,100$. The solid, dashed and dash-dot lines are for $v_{b}=10,20,100$ respectively.

Parameter used: $\mu=0, \nu_{0}=5 \mathrm{~Hz} . \ldots \ldots \ldots$

3.9 Variation of the normalized transmission function $\left|\nu_{1}(\omega) / \nu_{1 c}(0.1)\right|$ with increasing $\tau_{s}$ for $r=10,100$ when $\Delta \dot{v}=0$. Parameter used here and in the following: $\mu=0, v_{b}=10, \nu_{0}=5 \mathrm{~Hz} . \quad \ldots . .$.

3.10 Dependence of the normalized transmission function $\left|\nu_{1}(\omega) / \nu_{1}(0.1)\right|$ on $\tau_{s}$ for a current coded signal $(\mathrm{A}, \mathrm{C})$ and a noise coded signal $(\mathrm{B}, \mathrm{D}) . \mathrm{A}, \mathrm{B}, r=10 ; \mathrm{C}, \mathrm{D}, r=100 . \ldots \ldots$

3.11 Variation of the response $\left|\nu_{1 c}(\omega)\right|$ with increasing $\Delta \dot{v}$ for different $\tau_{s}$ and $r$.

3.12 Dependence of the transmission function $\left|\nu_{1}(\omega)\right|$ on the correlation time $\tau_{s}$ for different $\Delta \dot{v} . r$ is fixed to be 10 .

4.1 Illustration of the model. A, positive $r_{1}, \mathrm{~B}, r_{1}=0, \mathrm{C}$, negative $r_{1}$, $\mathrm{D}$, positive $r_{1}$ and negative $r$, a bistable system. . . . . . . .

4.2 Schematic illustration of exaggerating the edge (A) and cutting the edge (B) within the subthreshold region.

4.3 The stationary firing rate $\nu_{0}$ for the 3 -piece model corresponding to the cases of exaggerating the edge (A) and cutting the edge (B). 82

4.4 The normalized transmission functions $\left|\nu_{1}(\omega) / \nu_{1}(0.1)\right|$ for the 3piece model from exacting the edge, corresponding to Fig. 4.2 A.

Parameters: $v_{0}=0.8, r_{1}=-2,-1,0.5 \ldots \ldots \ldots$

4.5 The normalized transmission functions $\left|\nu_{1}(\omega) / \nu_{1}(0.1)\right|$ for the 3piece model from cutting the edge, corresponding to Fig. 4.2 B. Parameters: $v_{0}=0.8,0.6,0.4, r_{1}=0$.

4.6 Schematic illustration of manipulations for the spike initiation dynamics. A, $p_{0}$ is fixed and $r_{1}$ is varied. B, AP initiation threshold $v_{t 1}$, i.e., the zero crossing point of the piece 2 , is fixed and the slope $r_{1}$ is varied. $\mathrm{C}$, The middle piece is fixed and the slope $r$ of piece 2 is varied.

4.7 The normalized transmission functions $\left|\nu_{1}(\omega) / \nu_{1}(0.1)\right|$ for the 3piece model corresponding to Fig. 4.6A. Parameters used are: $v_{0}=0.6, r_{1}=0.5,2,5, r=10,100 \ldots \ldots \ldots \ldots$

4.8 The normalized transmission functions $\nu_{1}(\omega) / \nu_{1}(0.1)$ for the 3 piece model corresponding to Fig. 4.6 B. Parameters used are: $v_{t 1}=0.8, r_{1}=0.5,2,5$. 
4.9 The normalized transmission functions $\nu_{1}(\omega) / \nu_{1}(0.1)$ for the 3piece model corresponding to Fig. 4.6C. Parameters used are: $v_{0}=0.6, v_{1}=1$. For $\mathrm{A}$ and $\mathrm{B}, r_{1}=10, r=5,20,100$. Threshold crossing occurred within the middle piece (piece 3 ). For C and D, $r_{1}=0.5, r=5,10,100$. Threshold rossing occurred within the right piece (piece 2). . . . . . . . . . . . . . .

4.10 Illustration of fitting a 3-piece model to the EIF model. The left piece is from the leak current. The middle piece is tangent to the EIF model, so $\psi_{0}$ is fixed. The right piece passes the unstable fixed point $v_{t 0}$ with a slope chosen to fit the $f-I$ curve. Extending the left and right piece of the 3-piece model to cross at a point give a 2-piece model.

4.11 The $f-I$ curves for the EIF model and the 3-piece model. A, $f-I$ curves for a constant current; B, for a noisy current (Gaussian white noise, $\sigma=10 \mathrm{mV}$ ). The $f-I$ curve for the corresponding 2-piece model is also shown. The parameters for the EIF model are from experiments (Badel et al., 2008). . . . . . . . . . . . . .

4.12 Comparison of the linear response for the EIF model (from Badel et al. (2008)) and the fitted 3-piece model. Parameter: $\mu=$ $8.5 \mathrm{mV}, \sigma=10 \mathrm{mV}, \nu_{0}=10 \mathrm{~Hz}$. . . . . . . . . . .

4.13 Schematic illustration of the 3-piece model describing a bistable system with different $r_{1}$ and $r$. A, $r$ is fixed and $r_{1}$ is variable; $\mathrm{B}$,

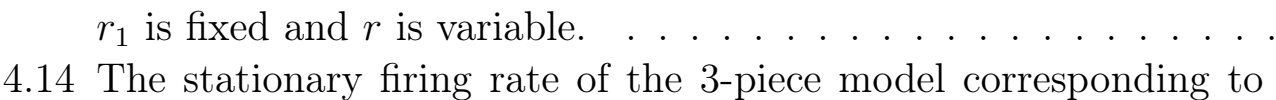
Fig. 4.13 with $v_{+1}=1$ and $v_{+0}=2 . \quad$ A. $r=-1$ and $r_{1}=$ 1,10 and $100 ; \mathrm{B}, r_{1}=10$ and $r=-1,-10$ and -100 . . . . .

4.15 The linear response of the 3-piece model for a current coded signal ( $\mathrm{A}$ and $\mathrm{C}$ ) and for a noise coded signal (B and $\mathrm{D})$ corresponding to Fig. 4.13 with $v_{t 1}=1$ and $v_{t 0}=2$. A. B. $r=-1$ and $r_{1}=$ 1,10 and $100 ; \mathrm{C}, \mathrm{D}, r_{1}=10$ and $r=-1,-10$ and $-100 \ldots$.

5.1 Illustration of the geometry and dynamics of the model used. A, the two-compartment model; B, the Gaussian neuron model; C, the correlation function for the membrane potential at the soma as given by Eq. (5.38): D. the membrane potential at soma, the

5.2 middle of the AIS and the end of the AIS. . . . . . . . . . . . (B) at soma on the position of AP evoked along the axon. Note that $\tau_{s}=10 \mathrm{~ms}$ corresponds to the simple ball-and-stick model. Parameters used: $\psi_{0}=10, \frac{\lambda_{2}}{\lambda_{1}}=1 . x_{1}=0.2 \lambda_{1}$. . . . . . . . 113

5.3 Dependence of transmission function on the membrane time constant $\tau_{1}$ at the AP initiation compartment for different coupling constant $c . \mathrm{A}, c=1 ; \mathrm{B}, c=5$. 
5.4 Dependence of transmission functions on the time constant $\tau_{1}$ at AP initiation compartment for different coupling constant $K$ and at different position of the AIS. For $\mathrm{A}$ and $\mathrm{B}, x=0.1$, in the middle of the AIS; C and D, $x=0.2$, at the end of AIS. Other parameters used are: $\tau_{2}=\tau_{m}=10 \mathrm{~ms}, \lambda_{2} / \lambda_{1}=1 \ldots \ldots . . . .115$

5.5 The same as Fig. 5.4. but with $\lambda_{2} / \lambda_{1}=10$. . . . . . . . . . . . 115 


\section{Chapter 1}

\section{Introduction}

Cortical neurons encode information into trains of action potential (AP). The spiking activities of individual cortical neurons are sparse and the average firing rates are verv low (several Hz or even lower) as revealed by experiments (Burns \& Webb, 1976; Destexhe \& Paré, 1999; Margrie et al., 2002; DeWeese et al., 2003) and theoretical estimate of the energy budget for the brain (Attwell \& Laughlin, 2001; Laughlin \& Sejnowski, 2003; Lennie, 2003). In general a strong degree of irregularity in the spike trains is observed and responses of single neurons to repeated sensorv stimuli show large variability (Softkv \& Koch. 1993: Shadlen \& Newsome, 1998; DeWeese et al., 2003). These characteristics limit the signal encoding ability of single neurons. Neuronal circuits, however, are able to function in a very fast way and can detect fast signal within a short time course as observed in behavioral experiments (Populin \& Yin, 1998; VanRullen \& Thorpe, 2001; Stanford et al., 2010). For example, monkeys are able to discriminate different colors in less than $30 \mathrm{~ms}$ (Stanford et al., 2010). To encode fast changing signals a large population of neurons is usually involved that can transmit the signal into its population averaged firing rate.

\section{Dynamical response of neuronal populations}

Cortical neurons with a low average firing rate can in principle encode fast changing signals through population response. The linear response theory of neuronal populations has been first formulated in Knight (1972a, b) and later generalized in Gerstner (2000) where artificial noise models were applied, e.g., a random chosen threshold value or resetting potential. The linear response theory for a population of more realistic cortical neuron models with realistic noise was developed recently (Brunel et al., 2001; Lindner \& Schimansky-Geier, 2001; Fourcaud \& Brunel, 2002; Fourcaud-Trocme et al., 2003; Naundorf et al., 2005). In such studies, the diffusion approximation has be applied to the large amount of poisson-like synaptic inputs each neuron receives. The synaptic input can therefore be divided 
into a mean input current part and a Gaussian noise part. If the finite duration of synaptic filtering is ignored, e.g. an instantaneous synapse, the synaptic noise can be approximated as a Gaussian white noise, otherwise a colored noise with finite correlation time provides a good description (Fourcaud \& Brunel, 2002). Each neuron in a population receives an independent realization of the Gaussian random noise. A signal received by the population can be encoded into both the mean current part and the noise part, which provide two separate channels in the population encoding. Traditionally, the mean input channel is the focus of attention for research (Brunel et al., 2001: Fourcaud-Trocme et al., 2003: Naundorf et al., 2005; (Köndgen et al., 2008). But recent experimental and theoretical studies have shown that both of the two encoding paradigms are important and the noise coded signal can even be faster and more efficiently (Lindner \& SchimanskyGeier, 2001; Silberberg et al., 2004; Boucsein et al., 2009). The linear response is an important characteristics of the dynamics of neuronal populations and is essential for the self-consistent mean field theory of network properties (see e.g. Geisler et al. (2005)). The aim of this thesis is to understand some important and intriguing aspects of the linear response of cortical neurons.

\section{High cutoff frequency in the dynamical response of neuronal ensembles}

Recent experiments have observed surprisingly high cutoff frequency for action potential encoding of cortical neurons driven bv fluctuating input currents (Köndgen et al., 2008; Boucsein et al., 2009; Higgs \& Spain, 2009). In a seminal paper Köndgen et al. (2008) showed that the transmission function of layer 5 pyramidal neurons for a noisy sinusoidal signal does not decay until about $200 \mathrm{~Hz}$. Later experiments confirmed such high cutoff frequencies for signals encoded in both the mean current and noise strength (Boucsein et al., 2009) and in other types of cortical neurons (Higgs \& Spain, 2009). In an early experiment fast response properties were observed also for step change in the stimuli (Silberberg et al., 2004). Previous theoretical studies of biophysical neuron models, however, predicted cutoff frequencies of the order of the average firing rate or the inverse membrane time constant (below $20 \mathrm{~Hz}$ ), much lower than experimental observed values (Fourcaud-Trocme et al., 2003: Fourcaud-Trocme \& Brunel, 2005: Naundorf et al., 2005). Thus, the origin of the high cutoff frequencies found in cortical neurons is currently not well understood. Numerical investigation of conductance based models and reduced one-variable neuron models suggested that details of AP generation can strongly influence the dynamical response of neuronal populations (Fourcaud-Trocme et al., 2003: Fourcaud-Trocme \& Brunel, 2005: Naundorf et al., 2005). What is missing, however, is a transparent understanding of how and when the population cutoff frequency can dissociate from the basic single 
neuron timescale set by the mean firing rate and the time constant of membrane potential relaxation.

\section{Unique features of AP initiation in cortical neurons}

Two characteristic features of the AP generation of cortical neurons have been revealed in experiments recently (Naundorf et al., 2006). In the $\dot{V}-V$ pseudo phase plane, where $V(t)$ is the membrane potential, the AP waveforms of cortical neuron were found to have a very fast onset rapidness and a large threshold variability. Extensive numerical simulation showed that these two features can not be achieved together in classical Hodgkin-Huxley type models with realistic parameters (Naundorf et al., 2006; Baranauskas et al., 2010) (but see also (McCormick et al., 2007; Yu et al., 2008)). One main assumption in HodgkinHuxley tvpe models is the independent opening of sodium channels (Hodgkin \& Huxley, 1952). A new model which introduced cooperativity of sodium channel opening among neighboring channels, was proposed to reproduce the two features (Naundorf et al., 2006). The hypothesis was supported in in vitro experiment by applying Tetrodotoxin (TTX) to reduce the sodium channel density. Both the amplitude of AP and its onset rapidness was effectively reduced when TTX was applied and by wash-out of TTX the fast onset reappeared, which support the existence of cooperativity among sodium channel opening. One of the aims of this thesis is to understand the functional implication of these unexpected features in the AP initiation of cortical neurons and to relate them to the dynamical response properties through analytical tractable neuron models.

\section{One-variable spiking neuron model}

There is a large class of one-variable integrate-and-fire (IF) neuron models extensively used for theoretical investigation and network simulations. The IF neuron has a long history since its introduction more than one century ago by Lapicque (Lapicque, 1907). These models capture, more or less, some basic aspects of AP generation in real neurons. In the long-standing Leaky integrate-and-fire (LIF) model, only the leak current is included. The model neuron integrates input and fires a spike when the membrane potential reaches a threshold value. The Quadratic IF model (QIF) or equivalently, the Theta neuron, captures the dynamics for type I neurons near the threshold (Ermentrout, 1996). The exponential IF (EIF) model, in which an exponential spike generating current is introduced to mimic the voltage dependent activation of sodium current, provides a good fit to more complex conductance based neuron models and to experimental data (Fourcaud-Trocme et al., 2003; Badel et al., 2008).

Since there is no restoring variable in the one-variable models, a reset mech- 
anism is usually adopted: when the membrane potential reaches some threshold value, the truncation point of the AP waveform, it is reset to a resetting potential after a possible refractory time. Therefore, an absorbing boundary condition needs to be assigned at the truncation point of APs in the neuron's phase space. In an IF neuron model, the absorbing boundary behaves as a point of no return which does not exist in the dynamics of real neurons and is always needed to be assigned, say it in infinity or at finite value. In the nonlinear IF models, e.g., the EIF and QIF models, the boundaries can be taken at infinity, since these models exhibit finite time blow-up. In the IF models with linear dynamics, e.g. the LIF model and the perfect integrator, finite boundaries are needed. Nevertheless, a finite absorbing boundary might introduce severe artifacts in the dynamical response of the model neurons. For example, the linear response of the LIF model to a noise coded signal with white noise or to a current coded signal with colored noise, shows a flat transmission function, which means the model neurons can follow an arbitrary high frequency signal faithfully. For the nonlinear IF models, the linear response to sinusoidal signals can only be solved numerically except for the high frequency limit, which were found to decay as power laws. We will introduce a new kind of one-variable spiking neuron model with piecewise linear dynamics and a finite threshold, in which the linear response can be solved analytically and the boundary induced artifacts can be separated out.

\section{Axonal initiation of action potential}

Real neurons are spatially extended objects and have complex morphology. Where APs are generated is important for synaptic integration and signal encoding. Although it has been known for a long time that APs are initiated in the axon, only recently the proximal axon was revealed to be the position of the AP initiation site of cortical neurons (Palmer \& Stuart, 2006). Many physiological properties at the axon initial segment (AIS) were found to be specifically arranged for the AP initiation and different from other parts of the neuron, e.g., a higher sodium channel density and specialized potassium channels (Yu et al., 2008; Kole et al., 2008; Fleidervish et al., 2010).

The functional implications of the AIS organization are not well understood yet. It has been claimed that the AIS can enhance coincident detection in auditory neurons (Kuba et al., 2006). The location and length of AIS were found to be plastic and influenced by experience (Grubb \& Burrone, 2010; Kuba et al., 2010). The back propagation to the soma of the APs initiated at AIS was suggested to be the reason for the fast $\mathrm{AP}$ onset and large variability of AP generation observed at soma (Yu et al., 2008), but this is still in controversy (Naundorf et al., 2007; Baranauskas et al., 2010).

In modeling spatially extended neurons, the complex morphology of real neurons could be simplified to a dendritic tree using the cable theory and further to 
a nerve cylinder described by the multi-compartment model (Rall, 1959, 1977). The cable equation has been applied in neuroscience as a simplified description of nerve cylinder for a long time (Hodgkin \& Rushton, 1946). The stochastic properties of depolarization of a passive nerve cylinder were studied for synaptic inputs homogeneous along the cable modeled as white noise or colored noise (Wan \& Tuckwell, 1979; Tuckwell \& Walsh, 1983; Tuckwell et al., 1984, 2002). To investigate the functional role of the AIS, we will further assume the membrane potential along the axon as a Gaussian random field with a given correlation function. By combing the Gaussian neuron model with the linear cable equation, the firing properties of a nerve cylinder could be investigated. In this way we can study the functional effect of AP initiation at the AIS theoretically.

\section{Structure of the thesis}

The thesis is organized as follows. In Chapter 2 the relation between the AP onset rapidness and the cutoff frequency in dynamical response is investigated assuming the external synaptic noise is a Gaussian white noise. In Chapter 3 we consider an intrinsic mechanism, i.e. cooperativity in the sodium channels opening, that produces a large AP onset rapidness and an external factor, i.e. correlation in the synaptic noise that is essential for obtaining a high cutoff frequency for a current coded signal. In Chapter 4 a general piecewise linear model is constructed which includes the models introduced in previous chapters as limit cases. It could be designed to match the stationary and dynamical response of the EIF model and allows one to extract the AP onset rapidness for cortical neurons. In Chapter 5 the signal encoding properties of spatially extended neurons are investigated by combining the Gaussian neuron model and the linear cable equation and the functional effect of axonal AP initiation is investigated. The last chapter is a summary and perspective opened up by the results in this thesis. 


\section{Chapter 2}

\section{Spike onset dynamics and population response}

In this chapter an analytically solvable model which explicitly describes the dynamical AP initiation process will be presented to investigate the relation between the AP onset rapidness and the cutoff frequency in the dynamical response of neuronal populations. A neuron initiates an AP if the membrane potential passes an unstable fixed point, the voltage threshold. In the leaky integrate-and-fire (LIF) model, for which the linear response is known analytically (Brunel et al., 2001; Lindner \& Schimansky-Geier, 2001), the unstable fixed point coincides with the absorbing boundary and a spike is triggered immediately when the membrane potential reaches this threshold. As a consequence, boundary induced artifacts dominate the response for high signal frequencies in the LIF model (FourcaudTrocme et al., 2003; Fourcaud-Trocme \& Brunel, 2005; Naundorf et al., 2005). One important advantage of our new model is that such boundary induced artifacts can be separated out mathematically, isolating the physically meaningful part of the response function. This allows us to obtain an explicit relation between the large AP onset rapidness (Naundorf et al., 2006) and the high cutoff frequency behaviors in the dynamical response of cortical neurons observed recently for both mean current coded signals and noise coded signals (Köndgen et al., 2008; Boucsein et al., 2009; Higgs \& Spain, 2009).

\subsection{An analytically solvable model with an unsta- ble fixed point}

The simplest voltage dynamics that exhibits both a stable fixed point (the resting potential) and an unstable fixed point (the voltage threshold) has a piecewise linear membrane current, composed of a leak current for low potential and a linear spike generating current for high potential (see Fig. 2.1A). The model (we 
call it $r-\tau_{m}$ model) is defined by the following Langevin equation

$$
\tau_{m} \dot{v}=f(v)+\mu+\sigma \eta(t)
$$

where

$$
\begin{aligned}
f(v) & =-v+\Theta\left(v-v_{0}\right)(r+1)\left(v-v_{0}\right) \\
& = \begin{cases}-v, \quad-\infty<v \leq v_{0} \\
r\left(v-v_{t}\right), & v_{0}<v \leq v_{b}\end{cases}
\end{aligned}
$$

Here $v$ is the membrane potential relative to the resting potential, $\tau_{m}$ is the membrane time constant, $r$ is the AP onset rapidness with $\frac{\tau_{m}}{r}$ representing the time constant for AP initiation, and $\Theta(v)$ is the Heaviside step function. $\mu$ is the mean input current and $\sigma$ is the amplitude of synaptic noise. $\eta(t)$ is a Gaussian white noise which satisfies $\langle\eta(t)\rangle=0$ and $\left\langle\eta(t) \eta\left(t^{\prime}\right)\right\rangle=\tau_{m} \delta\left(t-t^{\prime}\right)$. For convenience we take $\tau_{m}$ as the unit of time in analytical calculation.

A neuron receives a large amount of synaptic inputs and each one is very weak, therefore the diffusion approximation can be applied. A Gaussian white noise provides a good approximation if the postsynaptic current can be taken as a sum of delta pulses. The color noise case will be studied in next chapter, which plays an important role in understanding the high cutoff frequency for a current coded signal. The crossing point $v_{0}$ of the two pieces sets the rheobase current, which will be used as the unit of voltage, $v_{0}=1$. The threshold potential $v_{t}$ is related with $v_{0}$ by $v_{t}=(1+1 / r) v_{0}$. In Fig. $2.1 \mathrm{~B}$, traces of membrane potential with three different onset rapidness $r$ are shown. We can see that the larger is $r$, the faster is the spiking of the neuron. Distinct from the quadratic integrateand-fire (QIF) model and exponential integrate-and-fire (EIF) model, there is no finite time blow-up here. So we need to truncate the AP at some finite voltage $v_{b}$. When the membrane potential reaches $v_{b}$, it is reset to a voltage $v_{r}$ and stays there for an absolute refractory period $\tau_{r}$. Since we are mostly interested in the fluctuation driven regime with a low firing rate, the effect of the refractory period is not important and we will keep $\tau_{r}$ explicitly only in the analytical analysis, but take $\tau_{r}=0$ in numerical simulation.

The Fokker-Planck equation (FPE) corresponding to Eq. (2.1) has the following form (Risken, 1984)

$$
\begin{cases}\partial_{t} P_{1}(v, t)+\partial_{v}\left(-v+\mu-\frac{1}{2} \sigma^{2} \partial_{v}\right) P_{1}(v, t)=0, & -\infty<v \leq v_{0} \\ \partial_{t} P_{2}(v, t)+\partial_{v}\left(r\left(v-v_{t}\right)+\mu-\frac{1}{2} \sigma^{2} \partial_{v}\right) P_{2}(v, t)=0, & v_{0}<v \leq v_{b}\end{cases}
$$

where $P_{1}(v, t)$ and $P_{2}(v, t)$ are the probability densities $P(v, t)$ for the membrane potential $v$ located within $-\infty<v \leq v_{0}$ and $v_{0}<v \leq v_{b}$ respectively. 
A

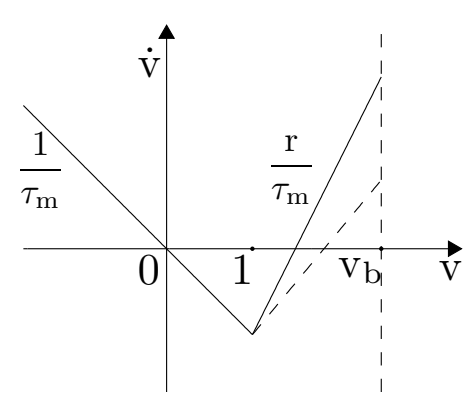

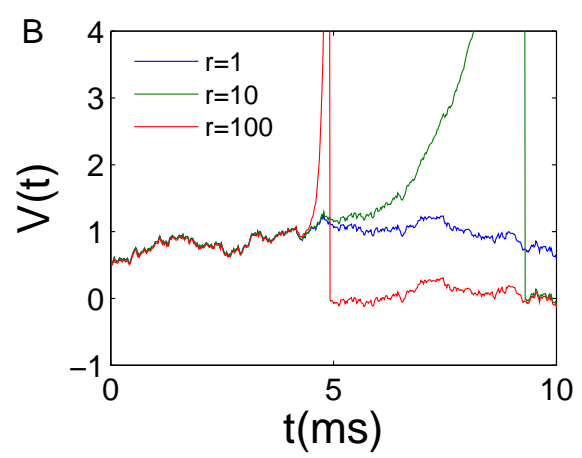

Figure 2.1: A, illustration of the model. B, $V(t)$ trajectories for identical noise and three different values of $\mathrm{r}$ in the noise driven regime.

Defining the probability currents $J(v, t)$ within two membrane potential ranges as

$$
\begin{aligned}
& J_{1}(v, t)=\left(-v+\mu-\frac{1}{2} \sigma^{2} \partial_{v}\right) P_{1}(v, t), \\
& J_{2}(v, t)=\left(r\left(v-v_{t}\right)+\mu-\frac{1}{2} \sigma^{2} \partial_{v}\right) P_{2}(v, t),
\end{aligned}
$$

the FPE then becomes the equation for probability conservation,

$$
\partial_{t} P(v, t)+\partial_{v} J(v, t)=0 .
$$

From the reset assumption, an absorbing boundary condition is assigned at $v_{b}$,

$$
P_{2}\left(v_{b}, t\right)=0
$$

and the probability current at the reset point $v_{r}$ has a jump

$$
J_{1}\left(v_{r}^{+}, t\right)-J_{1}\left(v_{r}^{-}, t\right)=J_{2}\left(v_{b}, t-\tau_{r}\right) .
$$

Since the probability density should be continuous at $v_{r}$,

$$
P_{1}\left(v_{r}^{+}, t\right)-P_{1}\left(v_{r}^{-}, t\right)=0,
$$

Eq. (2.7) implies

$$
\partial_{v} P_{1}\left(v_{r}^{+}, t\right)-\partial_{v} P_{1}\left(v_{r}^{-}, t\right)=\partial_{v} P_{2}\left(v_{b}, t-\tau_{r}\right) .
$$

In addition, the probability density and its first derivative should be continuous at $v_{0}$

$$
\begin{aligned}
P_{1}\left(v_{0}, t\right) & =P_{2}\left(v_{0}, t\right), \\
\partial_{v} P_{1}\left(v_{0}, t\right) & =\partial_{v} P_{2}\left(v_{0}, t\right) .
\end{aligned}
$$


Finally the normalization condition of the probability density requires that

$$
\lim _{v \rightarrow-\infty} P_{1}(v, t)=0 .
$$

With these boundary conditions the solution of the FPE (2.3) is uniquely determined. The instantaneous firing rate in the population is given by the probability current into the absorbing boundary

$$
\nu(t) \equiv J_{2}\left(v_{b}, t\right)=-\frac{1}{2} \sigma^{2} \partial_{v} P_{2}\left(v_{b}, t\right) .
$$

\section{$2.2 \quad$ Stationary solutions}

When the mean input current $\mu$ and noise amplitude $\sigma$ are constant, the system is homogeneous and possesses a stationary solution with a constant probability current, e.g. $J_{1}(v)=J_{2}(v)=\nu_{0}$, where $\nu_{0}$ is the stationary firing rate. We denote the stationary probability density $P_{0}(v)$ as $P_{01}(v)$ and $P_{02}(v)$ for the two pieces respectively, which satisfy

$$
\begin{array}{ll}
\left(-v+\mu-\frac{1}{2} \sigma^{2} \partial_{v}\right) P_{01}(v)=0, & -\infty<v \leq v_{r} \\
\left(-v+\mu-\frac{1}{2} \sigma^{2} \partial_{v}\right) P_{01}(v)=\nu_{0}, & v_{r}<v \leq v_{0} \\
\left(r\left(v-v_{t}\right)+\mu-\frac{1}{2} \sigma^{2} \partial_{v}\right) P_{02}(v)=\nu_{0}, & v_{0}<v \leq v_{b}
\end{array}
$$

Utilizing the boundary conditions Eq. (2.6 2.11), we solve Eq. (2.13) and obtain

$$
\begin{aligned}
P_{01}(v)= & \frac{\nu_{0}}{\sigma} e^{-\frac{1}{\sigma^{2}}(v-\mu)^{2}}\left(2 \int_{\frac{\max \left(v, v_{r}\right)-\mu}{\sigma}}^{\frac{v_{0}-\mu}{\sigma}} e^{y^{2}} d y\right. \\
& \left.+\sqrt{\frac{\pi}{r}} e^{\left(1+\frac{1}{r}\right) \frac{1}{\sigma^{2}}\left(v_{0}-\mu\right)^{2}}\left(\operatorname{Erfc}\left(\frac{\mu-v_{0}}{\sqrt{r} \sigma}\right)-\operatorname{Erfc}\left(\frac{\mu+\dot{v}_{b}}{\sqrt{r} \sigma}\right)\right)\right), \\
P_{02}(v)= & \frac{\nu_{0}}{\sigma} \sqrt{\frac{\pi}{r}} e^{\frac{1}{r \sigma^{2}}\left(\mu+r\left(v-v_{t}\right)\right)^{2}}\left(\operatorname{Erfc}\left(\frac{\mu+r\left(v-v_{t}\right)}{\sqrt{r} \sigma}\right)-\operatorname{Erfc}\left(\frac{\mu+\dot{v}_{b}}{\sqrt{r} \sigma}\right)\right),
\end{aligned}
$$

where $\dot{v}_{b}=r\left(v_{b}-v_{t}\right)$. From Fig. 2.2 we see that that a large $r$ make the probability higher for finding the membrane potential located around the resting potential, while multiplying the firing rate can increase the probability above the threshold. 

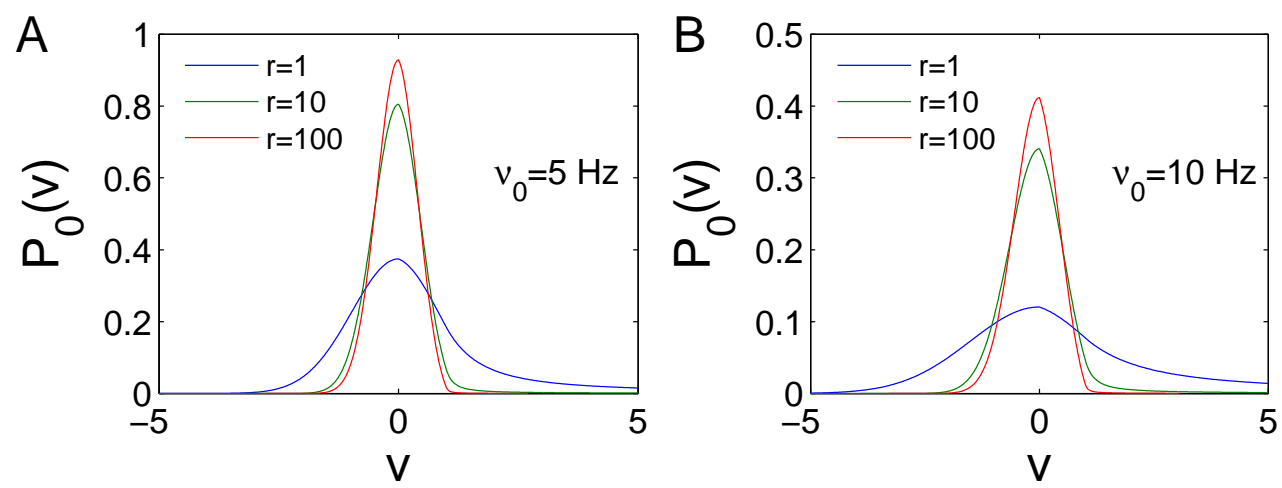

Figure 2.2: Dependence of the stationary probability density on the membrane potential with firing rate $5 \mathrm{~Hz}(\mathrm{~A})$ and $10 \mathrm{~Hz}(\mathrm{~B})$. Parameters used here and in other figures in this chapter: $\tau_{m}=10 \mathrm{~ms}, \mu=0, v_{r}=0, \tau_{r}=0$ and $v_{b}=10$ if not denoted otherwise.

The stationary firing rate $\nu_{0}$ can be obtained by using the normalization condition of the probability density, $\int_{-\infty}^{v_{b}} P_{0}(v) d v=1$,

$$
\begin{aligned}
\nu_{0}^{-1}= & \sqrt{\pi} \int_{\left(\mu-v_{0}\right) / \sigma}^{\left(\mu-v_{r}\right) / \sigma} e^{y^{2}} \operatorname{Erfc}(y) d y \\
& +\frac{\pi}{2 \sqrt{r}} e^{\left(1+\frac{1}{r}\right) \frac{1}{\sigma^{2}}\left(v_{0}-\mu\right)^{2}} \operatorname{Erfc}\left(\frac{\mu-v_{0}}{\sigma}\right)\left(\operatorname{Erfc}\left(\frac{\mu-v_{0}}{\sqrt{r} \sigma}\right)-\operatorname{Erfc}\left(\frac{\mu+\dot{v}_{b}}{\sqrt{r} \sigma}\right)\right) \\
& +\frac{\sqrt{\pi}}{r} \int_{\left(\mu-v_{0}\right) / \sqrt{r} \sigma}^{\left(\mu+\dot{v}_{b}\right) / \sqrt{r} \sigma} d x e^{x^{2}}\left(\operatorname{Erfc}(x)-\operatorname{Erfc}\left(\frac{\mu+\dot{v}_{b}}{\sqrt{r} \sigma}\right)\right)+\tau_{r}
\end{aligned}
$$

Fig. 2.3 shows the dependence of the stationary firing rate $\nu_{0}$ on $\mu$ and $\sigma$. We see that $\nu_{0}$ becomes insensitive to $r$ when $r$ is large $(r>10)$. When $r \rightarrow \infty$, the second and third terms in Eq. (2.15) vanish and $\nu_{0}$ converges to the stationary firing rate for the LIF model with the threshold at $v_{0}$,

$$
\nu_{0, \mathrm{LIF}}^{-1}=\sqrt{\pi} \int_{\left(\mu-v_{0}\right) / \sigma}^{\left(\mu-v_{r}\right) / \sigma} e^{y^{2}} \operatorname{Erfc}(y) d y+\tau_{r} .
$$

The dependence of the stationary firing rate on the truncation point $v_{b}$ is mainly through the third term, which has the limit behavior $\frac{1}{r} \log v_{b}$ and is suppressed when $r$ is large. From Fig. 2.4, we see the dependence of $\nu_{0}$ on $v_{b}$ is quite weak when $r$ is not small $(r>1)$ in the low rate regime.

For the probability density $P_{01}(v)$, the dependence on $v_{b}$ is mainly contained in the factor $\nu_{0}$ when $v_{b}$ is large. We define the dimensionless density $\tilde{P}_{01}(v)$, such that $P_{01}=\frac{\nu_{0}}{\sqrt{D}} \tilde{P}_{01}(v)$ where $D=\frac{1}{2} \sigma^{2}$ is the diffusion constant. In the large $v_{b}$ limit $\tilde{P}_{01}(v)$ does not depend on $v_{b}$. The values of the density and its derivative 

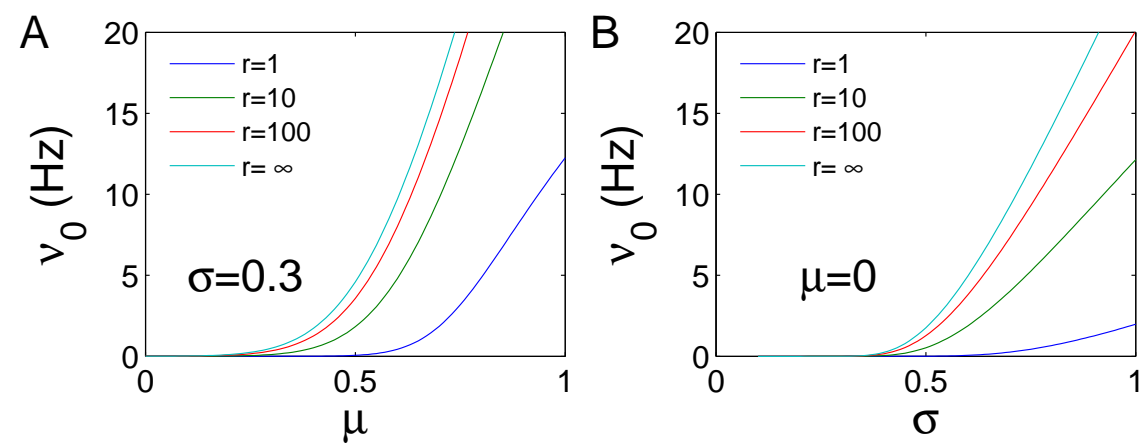

Figure 2.3: The dependence of stationary firing rate on the mean input current (A) and the noise strength (B) in the noise driven regime.
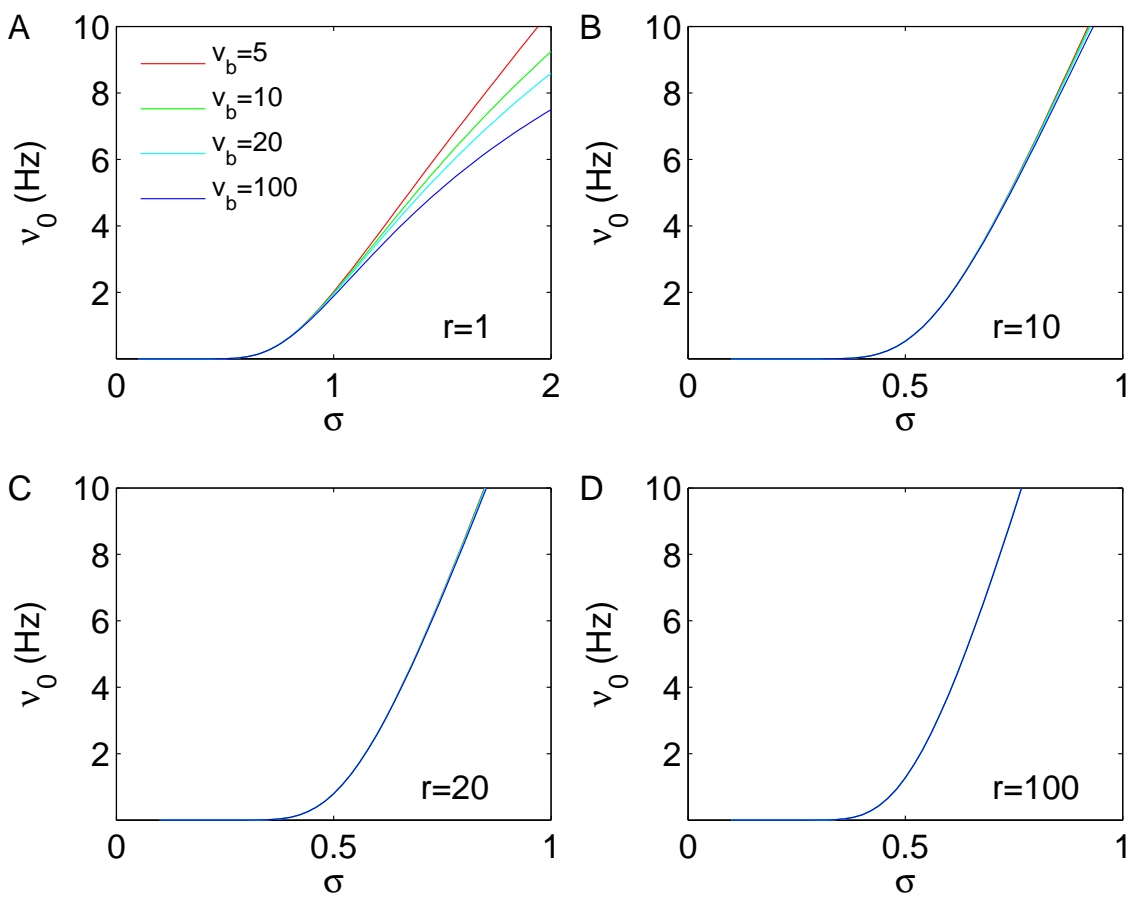

Figure 2.4: The dependence of stationary firing rate $\nu_{0}$ on the boundary $v_{b}$ for different $r$ with $\mu=0$. 


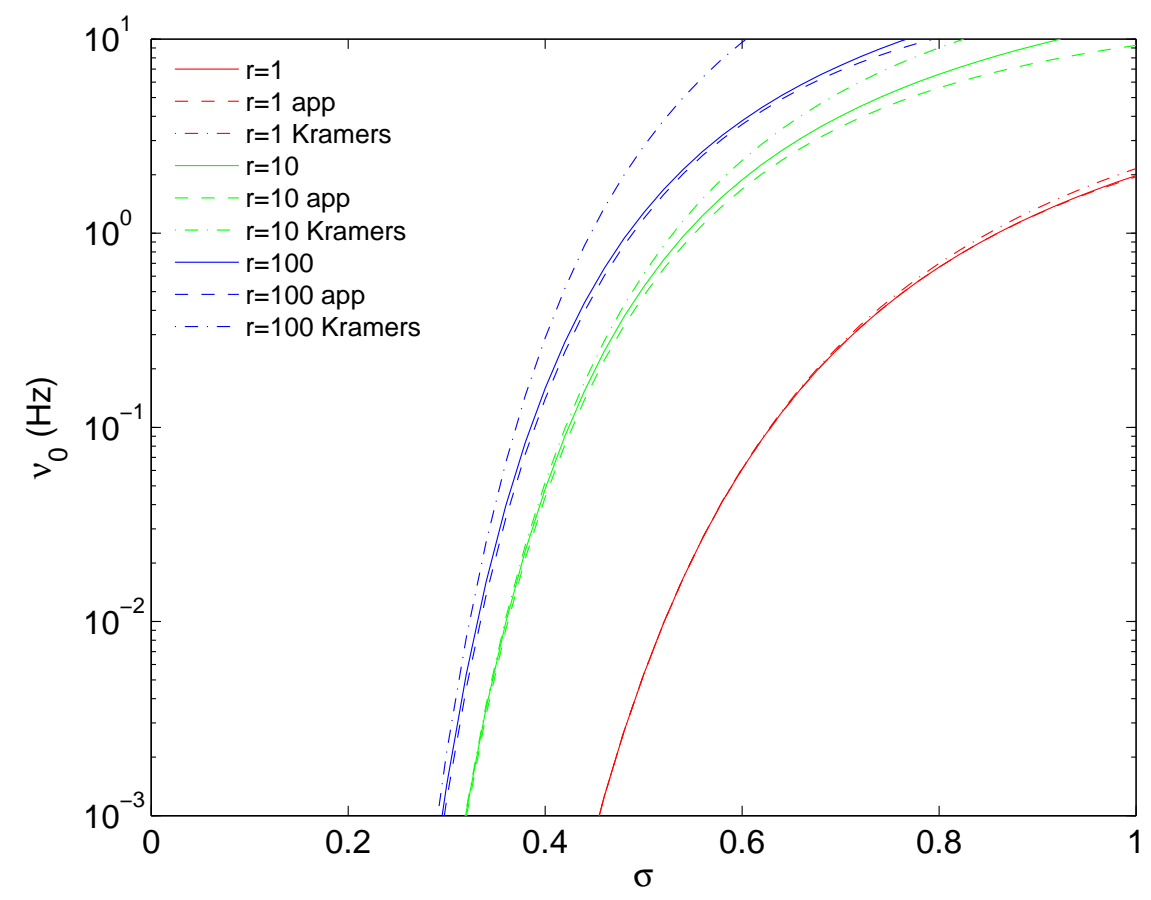

Figure 2.5: Comparison of the stationary firing rate $\nu_{0}$ with its approximation Eq. (2.18) (labeled as 'app') and the Kramers rate for different $r$.

at $v_{0}$ will be needed later in the study of linear response,

$$
\begin{aligned}
& \tilde{P}_{01}\left(v_{0}\right) \simeq \sqrt{\frac{\pi}{2 r}} e^{\frac{1}{r \sigma^{2}}\left(v_{0}-\mu\right)^{2}} \operatorname{Erfc}\left(-\frac{v_{0}-\mu}{\sqrt{r} \sigma}\right), \\
& \tilde{P}_{01}^{\prime}\left(v_{0}\right) \simeq-\frac{1}{\sqrt{D}}\left(1+\frac{v_{0}-\mu}{\sqrt{D}} \tilde{P}_{01}\left(v_{0}\right)\right) .
\end{aligned}
$$

For small $\sigma$, a simple approximate form can be found for the stationary firing rate in the noise driven regime $\mu<v_{0}$,

$$
\nu_{0}^{-1} \simeq \frac{\sqrt{\pi} \sigma}{v_{0}-\mu} e^{\frac{\left(v_{0}-\mu\right)^{2}}{\sigma^{2}}}+\frac{2 \pi}{\sqrt{r}} e^{\left(1+\frac{1}{r}\right) \frac{\left(v_{0}-\mu\right)^{2}}{\sigma^{2}}} .
$$

The second term in the above formula is the Kramers rate for the $r-\tau_{m}$ model when $r$ is finite. When $r$ is large the first term dominates and gives the Kramers rate for the corresponding LIF model with the threshold at $v_{0}$. In deriving Eq. (2.18) from Eq. (2.15), we have replaced the complementary Error functions with argument at $v_{0}$ in the second term with 2 , and applied a partial integration for the first term after replacing the Error function with 2, keeping only the leading term. Eq. (2.18) provides a good approximation when the firing rate is low and is better than the pure Kramers rate, as we can see from Fig. 2.5. The approximation is better for small and large $r$ than for medium $r$. 


\subsection{Linear responses and relation to the LIF model}

An input signal to a population of neurons can be encoded into two separate channels: the mean synaptic current and the amplitude of synaptic noise. In the $r-\tau_{m}$ model the linear response for both signal encoding paradigms can be solved analytically using the Green's function method. The solutions are expressed in terms of the parabolic cylinder functions defined in the appendix.

\subsubsection{Linear response to a current coded signal}

To obtain the linear response of the $r-\tau_{m}$ model for a mean current coded signal in the Fourier domain, we choose $\mu(t)=\mu+\varepsilon \cos (\omega t)$. The instantaneous firing rate can then be written as $\nu(t)=\nu_{0}+\varepsilon\left|\nu_{1 c}(\omega)\right| \cos \left(\omega t-\phi_{c}(\omega)\right)$ when $\varepsilon$ is small. Here $\left|\nu_{1 c}(\omega)\right|$ is the transmission function and $\phi_{c}(\omega)$ is the phase lag, both of which are functions of the signal frequency $\omega$.

The probability densities can be expanded as

$$
\begin{aligned}
& P_{1}(v, t)=P_{01}(v)+\varepsilon P_{11}(v, t)+\cdots, \\
& P_{2}(v, t)=P_{02}(v)+\varepsilon P_{12}(v, t)+\cdots,
\end{aligned}
$$

where '...' represents higher order terms in $\varepsilon$. The FPE at the first order in $\varepsilon$ is then given by

$$
\begin{aligned}
& \partial_{t} P_{11}(v, t)=\partial_{v}\left(v-\mu+D \partial_{v}\right) P_{11}(v, t)-\cos (\omega t) P_{01}^{\prime}(v), \quad-\infty<v \leq v_{0} \\
& \partial_{t} P_{12}(v, t)=\partial_{v}\left(-r v+r v_{t}-\mu+D \partial_{v}\right) P_{12}(v, t)-\cos (\omega t) P_{02}^{\prime}(v), v_{0}<v \leq v_{b}
\end{aligned}
$$

Factoring $P_{11}$ and $P_{12}$ as follows

$$
\begin{aligned}
& P_{11}(v, t)=\frac{1}{2}\left(e^{-i \omega t} q_{1}(v)+e^{i \omega t} q_{1}^{*}(v)\right) e^{-(v-\mu)^{2} / 4 D} \\
& P_{12}(v, t)=\frac{1}{2}\left(e^{-i \omega t} q_{2}(v)+e^{i \omega t} q_{2}^{*}(v)\right) e^{\left(r v^{2}-2\left(r v_{t}-\mu\right) v\right) / 4 D}
\end{aligned}
$$

where the asterisks represent complex conjugate, we obtain

$$
\begin{aligned}
& D q_{1}^{\prime \prime}(v)-\left(\frac{(v-\mu)^{2}}{4 D}-i \omega-\frac{1}{2}\right) q_{1}(v)=e^{(v-\mu)^{2} / 4 D} P_{01}^{\prime}(v), \\
& \frac{D}{r} q_{2}^{\prime \prime}(v)-\left(\frac{\left(v-v_{t}+\mu / r\right)^{2}}{4 D / r}-\frac{i \omega}{r}+\frac{1}{2}\right) q_{2}(v)=\frac{1}{r} e^{-\left(r v^{2}-2\left(r v_{t}-\mu\right) v\right) / 4 D} P_{02}^{\prime}(v) .
\end{aligned}
$$


Substituting Eq. (2.19) and Eq. (2.21) into the boundary conditions Eq. (2.6. 2.11), we see that $q_{1}$ and $q_{2}$ satisfy the following boundary conditions,

$$
\begin{aligned}
\lim _{v \rightarrow-\infty} q_{1}(v) & =0, \\
q_{2}\left(v_{b}\right) & =0, \\
q_{1}\left(v_{0}\right) & =q_{2}\left(v_{0}\right) e^{\Delta_{02}}, \\
q_{1}^{\prime}\left(v_{0}\right) & =q_{2}^{\prime}\left(v_{0}\right) e^{\Delta_{02}}, \\
q_{1}\left(v_{r}^{+}\right)-q_{1}\left(v_{r}^{-}\right) & =0 \\
q_{1}^{\prime}\left(v_{r}^{+}\right)-q_{1}^{\prime}\left(v_{r}^{-}\right) & =q_{2}^{\prime}\left(v_{b}\right) e^{\Delta_{01}+i \omega \tau_{r}}
\end{aligned}
$$

where

$$
\begin{aligned}
& \Delta_{01}=\left(\left(v_{r}-\mu\right)^{2}+\left(r v_{b}^{2}-2\left(r v_{t}-\mu\right) v_{b}\right)\right) / 4 D, \\
& \Delta_{02}=\left(\left(v_{0}-\mu\right)^{2}+\left(r v_{0}^{2}-2\left(r v_{t}-\mu\right) v_{0}\right)\right) / 4 D .
\end{aligned}
$$

The left sides of Eq. (2.22) have the form of parabolic cylinder equations. Eq. (2.22) can then be solved using the Green's function method with the solutions expressed using the parabolic cylinder functions. The Green's functions, denoted as $g_{1}(v, y)$ and $g_{2}(v, y)$, which are solutions of the following equations

$$
\begin{aligned}
& D g_{1}^{\prime \prime}-\left(\frac{(v-\mu)^{2}}{4 D}-i \omega-\frac{1}{2}\right) g_{1}=\delta(v-y), \\
& \frac{D}{r} g_{2}^{\prime \prime}-\left(\frac{\left(v-v_{t}+\mu / r\right)^{2}}{4 D / r}-\frac{i \omega}{r}+\frac{1}{2}\right) g_{2}=\delta(v-y),
\end{aligned}
$$

are given by

$$
\begin{aligned}
& g_{1}(v, y)=-\frac{1}{D} \begin{cases}\psi_{2}(y) \psi_{1}(v), & v<y \\
\psi_{1}(y) \psi_{2}(v), & y \leq v<v_{0}\end{cases} \\
& g_{2}(v, y)=\frac{r}{\psi_{3}\left(v_{b}\right) D} \begin{cases}Y_{1}(y) \psi_{3}(v), & v_{0} \leq v<y \\
\psi_{3}(y) Y_{1}(v), & y \leq v<v_{b}\end{cases}
\end{aligned}
$$

which satisfy the boundary conditions Eq. (2.23) and Eq. (2.24). Note that $\phi_{2}(v)$ will not appear in the final expressions. 
The solutions of Eq. (2.22) are given by

$$
\begin{aligned}
q_{1}(v)= & -\frac{1}{D}\left(\psi_{2}(v) \int_{-\infty}^{v} \psi_{1}(y) P_{01}^{\prime} e^{(y-\mu)^{2} / 4 D} d y\right. \\
& \left.+\psi_{1}(v) \int_{v}^{v_{0}} \psi_{2}(y) P_{01}^{\prime} e^{(y-\mu)^{2} / 4 D} d y\right) \\
& +k_{1}\left\{\begin{array}{l}
\psi_{2}\left(v_{r}\right) \psi_{1}(v), \quad v<v_{r} \\
\psi_{1}\left(v_{r}\right) \psi_{2}(v)+a\left(\psi_{2}\left(v_{r}\right) \psi_{1}(v)-\psi_{1}\left(v_{r}\right) \psi_{2}(v)\right), \quad v_{r} \leq v<v_{0}
\end{array}\right. \\
q_{2}(v)= & \frac{1}{\psi_{3}\left(v_{b}\right) D}\left(Y_{1}(v) \int_{v_{0}}^{v} \psi_{3}(y) P_{02}^{\prime}(y) e^{-\left(r y^{2}-2\left(r v_{t}-\mu\right) y\right) / 4 D} d y\right. \\
& \left.+\psi_{3}(v) \int_{v}^{v_{b}} Y_{1}(y) P_{02}^{\prime}(y) e^{-\left(r y^{2}-2\left(r v_{t}-\mu\right) y\right) / 4 D} d y\right) \\
& +k_{2} Y_{1}(v), v_{0}<v \leq v_{b}
\end{aligned}
$$

$k_{1}, k_{2}$ and $a$ are fixed by the boundary conditions (2.25) 2.28). We then have

$$
q_{2}^{\prime}\left(v_{b}\right)=-\frac{1}{D} e^{-\Delta_{01}-i \omega \tau_{r}}(A+B)\left(\psi_{1}\left(v_{r}\right)+\left(Y_{1} \psi_{1}^{\prime}-Y_{1}^{\prime} \psi_{1}\right) e^{\Delta_{1}-\Delta_{0}-i \omega \tau_{r}}\right)^{-1}
$$

with

$$
\begin{aligned}
A= & \int_{-\infty}^{v_{0}} \psi_{1}(y) P_{01}^{\prime} e^{(y-\mu)^{2} / 2 \sigma^{2}} d y \\
= & \frac{1}{1-i \omega} e^{\left(v_{0}-\mu\right)^{2} / 4 D}\left(\psi_{1} P_{01}-\sqrt{D} i \omega \Phi_{1} P_{01}^{\prime}-\frac{\nu_{0}}{\sqrt{D}} i \omega \Phi_{1}\left(v_{r}\right) e^{\Delta_{0}+i \omega \tau_{r}}\right) \\
B= & \frac{e^{\Delta_{02}}}{\psi_{3}\left(v_{b}\right)} \int_{v_{0}}^{v_{b}}\left(\left(\psi_{3} \psi_{1}^{\prime}-\psi_{3}^{\prime} \psi_{1}\right) Y_{1}(y)\right. \\
& \left.-\left(Y_{1} \psi_{1}^{\prime}-Y_{1}^{\prime} \psi_{1}\right) \psi_{3}(y)\right) P_{02}^{\prime}(y) e^{-\left(r y^{2}-\left(r v_{t}-\mu\right) y\right) / 4 D} d y \\
= & \frac{e^{\left(v_{0}-\mu\right)^{2} / 4 D}}{1+i \omega / r}\left(-\psi_{1} P_{01}-\sqrt{D} \frac{i \omega}{r} \Phi_{1} P_{01}^{\prime}+\frac{\nu_{0}}{\sqrt{r D}} e^{\Delta_{1}}\left(Y_{2} \psi_{1}^{\prime}-Y_{2}^{\prime} \psi_{1}\right)\right)
\end{aligned}
$$

where

$$
\begin{aligned}
\Delta_{0} & =\left(\left(v_{r}-\mu\right)^{2}-\left(v_{0}-\mu\right)^{2}\right) / 4 D=\left(v_{0}-v_{r}\right)\left(2 \mu-v_{0}-v_{r}\right) / 4 D \\
\Delta_{1} & =\left(\left(r v_{0}^{2}-2\left(r v_{t}-\mu\right) v_{0}\right)-\left(r v_{b}^{2}-2\left(r v_{t}-\mu\right) v_{b}\right)\right) / 4 D \\
& =\left(v_{0}-v_{b}\right)\left(2 \mu-v_{0}-\dot{v}_{b}\right) / 4 D
\end{aligned}
$$

In evaluating $A$ and $B$ we have used the recurrence relations of parabolic cylinder functions presented in the appendix and the fact that $\hat{P}_{01}(v) \equiv P_{01}(v) e^{(v-\mu)^{2} / 4 D}$ 
and $\hat{P}_{02}(v) \equiv P_{02}(v) e^{-\left(r v^{2}-\left(r v_{t}-\mu\right) v\right) / 4 D}$ satisfy the parabolic cylinder equation:

$$
\begin{aligned}
& D \hat{P}_{01}^{\prime \prime}(v)-\left(\frac{(v-\mu)^{2}}{4 D}-\frac{1}{2}\right) \hat{P}_{01}(v)=0 \\
& \frac{D}{r} \hat{P}_{02}^{\prime \prime}(v)-\left(\frac{\left(v-v_{t}+\mu / r\right)^{2}}{4 D / r}+\frac{1}{2}\right) \hat{P}_{02}(v)=0
\end{aligned}
$$

as can be easily proved from Eq. (2.13), so we have

$$
\begin{aligned}
& \hat{P}_{01}(v) \propto U\left(-\frac{1}{2},-\frac{v-\mu}{\sqrt{D}}\right), \\
& \hat{P}_{02}(v) \propto U\left(\frac{1}{2},-\frac{v-v_{t}+\mu / r}{\sqrt{D / r}}\right) .
\end{aligned}
$$

Therefore, they are parabolic cylinder functions with the same arguments as $\psi_{1}(v)$ and $\psi_{3}(v), \psi_{4}(v)$ respectively. The integration in $A$ and $B$ can then be performed by partial integration as is usually done for orthogonal functions and only some surface terms are left.

The constants $\Delta_{0}$ and $\Delta_{1}$ have physical meaning in the Kramers approximation of the model. They measure the ratio of the potential difference between $v_{r}$ and $v_{0}$ or $v_{0}$ and $v_{b}$, and the diffusion constant $D$ respectively.

At linear order in $\varepsilon$, the instantaneous firing rate is

$$
\begin{aligned}
\nu(t) & =-D \partial_{v} P\left(v_{b}, t\right) \\
& =\nu_{0}-\frac{\varepsilon}{2} D\left(e^{-i \omega t} q_{2}^{\prime}\left(v_{b}\right)+e^{i \omega t} q_{2}^{*}\left(v_{b}\right)\right) e^{\left(r v_{b}^{2}-2\left(r v_{t}-\mu\right) v_{b}\right) / 4 D} \\
& =\nu_{0}+\varepsilon\left|\nu_{1 c}(\omega)\right| \cos \left(\omega t-\phi_{c}(\omega)\right)
\end{aligned}
$$

where $\nu_{1 c}(\omega)=-D q_{2}^{\prime}\left(v_{b}\right) e^{\left(r v_{b}^{2}-2\left(r v_{t}-\mu\right) v_{b}\right) / 4 D}$ is the complex response function. The absolute value of $\nu_{1 c}(\omega)$ is the transmission function, while its phase angle gives the phase lag $\phi_{c}(\omega)$ of the linear response, $\phi_{c}(\omega)=\arg \left(\nu_{1 c}(\omega)\right) . \quad \nu_{1 c}(\omega)$ can be decomposed into two parts,

$$
\nu_{1 c}(\omega)=\nu_{1 c}^{L o w}(\omega)+\nu_{1 c}^{H i g h}(\omega)
$$

with

$$
\begin{aligned}
\nu_{1 c}^{\text {Low }}(\omega)= & \frac{\nu_{0}}{\sqrt{D}} \frac{i \omega}{(1-i \omega)(1+i \omega / r)} \times \\
& \frac{(1+1 / r)\left(\psi_{1} \tilde{P}_{01}-\sqrt{D} \Phi_{1} \tilde{P}_{01}^{\prime}\right)-(1+i \omega / r) \Phi_{1}\left(v_{r}\right) e^{\Delta_{0}}}{\psi_{1}\left(v_{r}\right) e^{\Delta_{0}+i \omega \tau_{r}}+\left(Y_{1} \psi_{1}^{\prime}-Y_{1}^{\prime} \psi_{1}\right) e^{\Delta_{1}}} \\
\nu_{1 c}^{\text {High }}(\omega)= & \frac{\nu_{0}}{\sqrt{r D}} \frac{1}{1+i \omega / r} \frac{\left(Y_{2} \psi_{1}^{\prime}-Y_{2}^{\prime} \psi_{1}\right) e^{\Delta_{1}}}{\psi_{1}\left(v_{r}\right) e^{\Delta_{0}+i \omega \tau_{r}}+\left(Y_{1} \psi_{1}^{\prime}-Y_{1}^{\prime} \psi_{1}\right) e^{\Delta_{1}}} .
\end{aligned}
$$




\subsubsection{Linear response to a noise coded signal}

For a noise coded signal, we have $\sigma(t)=\sigma+\varepsilon \cos (\omega t)$. Similar to the current coded signal case, the probability densities can be divided into

$$
\begin{aligned}
& P_{1}(v, t)=P_{01}(v)+\varepsilon \sigma P_{21}(v, t)+\cdots, \\
& P_{2}(v, t)=P_{02}(v)+\varepsilon \sigma P_{22}(v, t)+\cdots,
\end{aligned}
$$

and the FPE become

$$
\begin{aligned}
& \partial_{t} P_{21}(v, t)=\partial_{v}\left(v-\mu+D \partial_{v}\right) P_{21}(v, t)+\cos (\omega t) P_{01}^{\prime \prime}(v), \quad-\infty<v \leq v_{0} \\
& \partial_{t} P_{22}(v, t)=\partial_{v}\left(-r v+r v_{t}-\mu+D \partial_{v}\right) P_{22}(v, t)+\cos (\omega t) P_{02}^{\prime \prime}(v), v_{0}<v<v_{b}
\end{aligned}
$$

Factoring the densities as

$$
\begin{aligned}
& P_{21}(v, t)=\frac{1}{2}\left(e^{-i \omega t} q_{1}(v)+e^{i \omega t} q_{1}^{*}(v)\right) e^{-(v-\mu)^{2} / 4 D} \\
& P_{22}(v, t)=\frac{1}{2}\left(e^{-i \omega t} q_{2}(v)+e^{i \omega t} q_{2}^{*}(v)\right) e^{\left(r v^{2}-2\left(r v_{t}-\mu\right) v\right) / 4 D}
\end{aligned}
$$

we then get the following equations

$$
\begin{aligned}
& D q_{1}^{\prime \prime}(v)-\left(\frac{(v-\mu)^{2}}{4 D}-i \omega-\frac{1}{2}\right) q_{1}(v)=-e^{(v-\mu)^{2} / 4 D} P_{01}^{\prime \prime}(v), \\
& \frac{D}{r} q_{2}^{\prime \prime}(v)-\left(\frac{\left(v-v_{t}+\mu / r\right)^{2}}{4 D / r}-\frac{i \omega}{r}+\frac{1}{2}\right) q_{2}(v)=-\frac{1}{r} e^{-\left(r v^{2}-2\left(r v_{t}-\mu\right) v\right) / 4 D} P_{02}^{\prime \prime}(v) .
\end{aligned}
$$

The solutions of Eq. (2.44) are obtained by the Green's function method described in the case of a current coded signal,

$$
\begin{aligned}
& q_{1}(v)= \frac{1}{D}\left(\psi_{2}(v) \int_{-\infty}^{v} \psi_{1}(y) P_{01}^{\prime \prime} e^{(y-\mu)^{2} / 4 D} d y+\psi_{1}(v) \int_{v}^{v_{0}} \psi_{2}(y) P_{01}^{\prime \prime} e^{(y-\mu)^{2} / 4 D} d y\right) \\
&+k_{1}\left\{\begin{array}{l}
\psi_{2}\left(v_{r}\right) \psi_{1}(v), \quad v \leq v_{r} \\
\psi_{1}\left(v_{r}\right) \psi_{2}(v)+a\left(\psi_{2}\left(v_{r}\right) \psi_{1}(v)-\psi_{1}\left(v_{r}\right) \psi_{2}(v)\right), \quad v_{r}<v \leq v_{0}
\end{array}\right. \\
& q_{2}(v)=-\frac{1}{\psi_{3}\left(v_{b}\right) D}\left(Y_{1}(v) \int_{v_{0}}^{v} \psi_{3}(y) P_{02}^{\prime \prime}(y) e^{-\left(r y^{2}-2\left(r v_{t}-\mu\right) y\right) / 4 D} d y\right. \\
&\left.+\psi_{3}(v) \int_{v}^{v_{b}} Y_{1}(y) P_{02}^{\prime \prime}(y) e^{-\left(r y^{2}-2\left(r v_{t}-\mu\right) y\right) / 4 D} d y\right) \\
& \quad+k_{2} Y_{1}(v), \quad v \leq v_{b}
\end{aligned}
$$


where $q_{1}$ and $q_{2}$ satisfy the boundary conditions (2.25),2.28) as for a current coded signal. Solving $k_{1}, k_{2}$ and $a$, we obtain

$$
\begin{aligned}
q_{2}^{\prime}\left(v_{b}\right)= & \frac{1}{D} e^{-\Delta_{01}-i \omega \tau_{r}}\left(\psi_{1}\left(v_{r}\right)+\left(Y_{1} \psi_{1}^{\prime}-Y_{1}^{\prime} \psi_{1}\right) e^{\Delta_{02}-\Delta_{01}-i \omega \tau_{r}}\right)^{-1} \\
& \times\left(\frac{\nu_{0}}{D} \psi_{1}\left(v_{r}\right) e^{\left(v_{r}-\mu\right)^{2} / 4 D}+A+B\right)
\end{aligned}
$$

where

$$
\begin{aligned}
A= & \int_{-\infty}^{v_{0}} \psi_{1}(y) P_{10}^{\prime \prime} e^{(y-\mu)^{2} / 2 \sigma^{2}} d y \\
= & e^{\left(v_{0}-\mu\right)^{2} / 4 D}\left(P_{01}^{\prime} \psi_{1}+\frac{i \omega}{2-i \omega}\left(\frac{1}{\sqrt{D}} P_{01} \Phi_{1}\right.\right. \\
& \left.\left.\quad-(i \omega-1)\left(\Upsilon_{1} P_{01}^{\prime}+\frac{\nu_{0}}{D} \Upsilon_{1}\left(v_{r}\right) e^{\Delta_{0}+i \omega \tau_{r}}\right)\right)\right), \\
B= & \frac{e^{\Delta_{02}}}{\psi_{3}\left(v_{b}\right)} \int_{v_{0}}^{v_{b}}\left(\left(\psi_{3} \psi_{1}^{\prime}-\psi_{3}^{\prime} \psi_{1}\right) Y_{1}(y)\right. \\
= & e^{\left(v_{0}-\mu\right)^{2} / 4 D}\left(-\left(Y_{1} \psi_{1}^{\prime}-Y_{1}^{\prime} \psi_{1}\right) \psi_{3}(y)\right) P_{02}^{\prime \prime}(y) e^{-\left(r y^{2}-\left(r v_{t}-\mu\right) y\right) / 4 D} d y \\
& +\frac{1}{2+i \omega / r} e^{\Delta_{1}}\left(Y_{3} \psi_{1}^{\prime}-Y_{3}^{\prime} \psi_{1}\right) \\
& \left.\left.-\frac{i \omega}{\sqrt{D}} \Phi_{1} P_{01}-\frac{1}{r}(i \omega-1) i \omega \Upsilon_{1} P_{01}^{\prime}-\frac{\nu_{0}}{D} e^{\Delta_{1}}\left(Y_{3} \psi_{1}^{\prime}-Y_{3}^{\prime} \psi_{1}\right)\right)\right) .
\end{aligned}
$$

The instantaneous firing rate is given by

$$
\begin{aligned}
\nu(t) & =-\left.(D+\varepsilon \sigma \cos (\omega t)) \partial_{v}\left(P_{02}+\varepsilon \sigma P_{22}\right)\right|_{v_{b}} \\
& =\nu_{0}+\varepsilon \sigma\left|\nu_{1 n}(\omega)\right| \cos \left(\omega t-\phi_{n}(\omega)\right),
\end{aligned}
$$

where $\nu_{1 n}(\omega)$ is the complex response function given by

$$
\nu_{1 n}(\omega)=\frac{\nu_{0}}{D}-D q_{2}^{\prime}\left(v_{b}\right) e^{\left(r v_{b}^{2}-2\left(r v_{t}-\mu\right) v_{b}\right) / 4 D}
$$

and $\phi_{n}(\omega)=\arg \left(\nu_{1 n}(\omega)\right)$ is the phase lag. Again $\nu_{1 n}(\omega)$ can be decomposed into two parts,

$$
\nu_{1 n}(\omega)=\nu_{1 n}^{\text {Low }}(\omega)+\nu_{1 n}^{\text {High }}(\omega)
$$

with

$$
\begin{aligned}
\nu_{1 n}^{\text {Low }}(\omega)= & \frac{\nu_{0}}{D} \frac{i \omega(i \omega-1)}{(2-i \omega)(2+i \omega / r)} \\
& \times \frac{(1+1 / r)\left(\frac{i \omega}{1-i \omega} \phi_{1} \tilde{P}_{01}+2 \sqrt{D} \Upsilon \tilde{P}_{01}^{\prime}\right)+(2+i \omega / r) \Upsilon\left(v_{r}\right) e^{\Delta_{0}}}{\psi_{1}\left(v_{r}\right) e^{\Delta_{0}+i \omega \tau_{r}}+\left(Y_{1} \psi_{1}^{\prime}-Y_{1}^{\prime} \psi_{1}\right) e^{\Delta_{1}}} \\
\nu_{1 n}^{H i g h}(\omega)= & \frac{\nu_{0}}{D} \frac{1}{2+i \omega / r} \frac{\left(Y_{3} \psi_{1}^{\prime}-Y_{3}^{\prime} \psi_{1}\right) e^{\Delta_{1}}}{\psi_{1}\left(v_{r}\right) e^{\Delta_{0}+i \omega \tau_{r}}+\left(Y_{1} \psi_{1}^{\prime}-Y_{1}^{\prime} \psi_{1}\right) e^{\Delta_{1}}}
\end{aligned}
$$




\subsubsection{Evaluation of the results}

The analytical expressions for $\nu_{1}(\omega)$ can be easily evaluated using Mathematica, where the Whittake $D$ function $D_{-a-\frac{1}{2}}(x)=U(a, x)$ is implemented. The function $V(a, x)$ can then be evaluated using the relation

$$
\pi V(a, x)=\Gamma\left(\frac{1}{2}+a\right)(\sin \pi a U(a, x)+U(a,-x)) .
$$

See the appendix for more details of the properties of parabolic cylinder functions. Note that in evaluating such expressions, large numbers will appear and then cancel with each other. To do this accurately, one needs to reset the precision of calculation in Mathematica to a higher value, otherwise one will observe artificial oscillations at high signal frequencies. For the figures here, the maximum number of extra digits of precision was set to be 1000. Numerical simulations were performed to generate spike trains within a fixed duration $T$ using the second order Runge-Kutta method (Honeycutt, 1992a). The complex response function $\nu_{1 c}(\omega)$ is then given by

$$
\nu_{1 c}(\omega)=\frac{2}{\varepsilon N_{e} T} \sum_{i=1}^{N_{e}} \sum_{j=1}^{N_{s}} e^{-i \omega t_{j}^{i}}
$$

where $N_{e}$ is the number of trials, $N_{s}$ the number of spikes in the duration $T$ in one trial, $t_{j}^{i}$ the $j$ th spike in the $i$ th trial, and $\varepsilon$ the amplitude of input signal. The duration $T$ for each trial was taken such that the average number of spikes $\bar{N}_{s}$ is $\bar{N}_{s}=10^{6}$ according to the relation $T=\bar{N}_{s} / \nu_{0}$ for both signal encoding paradigms. For numerical calculations of $\nu_{1 n}(\omega)$, we use the following formula,

$$
\nu_{1 n}(\omega)=\frac{2}{\varepsilon \sigma N_{e} T} \sum_{i=1}^{N_{e}} \sum_{j=1}^{N_{s}} e^{-i \omega t_{j}^{i}}
$$

Note that compared with the case for a current coded signal, there is an additional factor $\frac{1}{\sigma}$ from the definition (2.41).

In Fig. 2.6 2.8, we plot the linear response for different $r$. Note that the frequency $f$ in the figures are related to the angular frequency $\omega$ here by $\omega=2 \pi f$. We see that $\nu_{1}^{\text {Low }}(\omega)$ dominate for the low and intermediate frequency range, while the $\nu_{1}^{\text {High }}(\omega)$ parts are responsible for the high frequency limit. Here and in the following, both cases are referred to when the subscripts ' $c$ ' and ' $n$ ' are dropped. In fact, as shown later, the $\nu_{1}^{H i g h}(\omega)$ parts reflect mainly the artifacts from the absorbing boundary condition. It is the $\nu_{1}^{\text {Low }}(\omega)$ parts which are physically relevant and reflect the influence of onset dynamics on the dynamical response. 

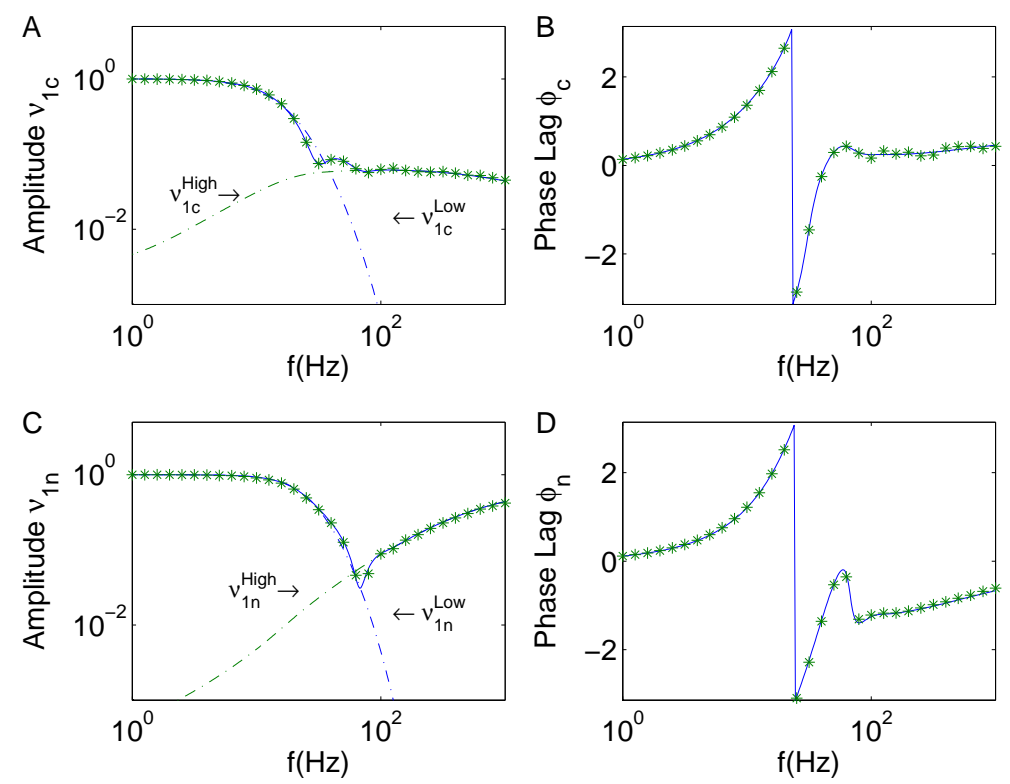

Figure 2.6: The normalized transmission functions $\nu_{1}(\omega) / \nu_{1}(0.1)$ and phase lags for a current coded signal (A,B) and for a noise coded signal (C,D) with $r=1$ and $\nu_{0}=5 \mathrm{~Hz}$. The dashed and solid lines are theoretical results and the points are from numerical simulation using Eq. (2.53) and (2.54).
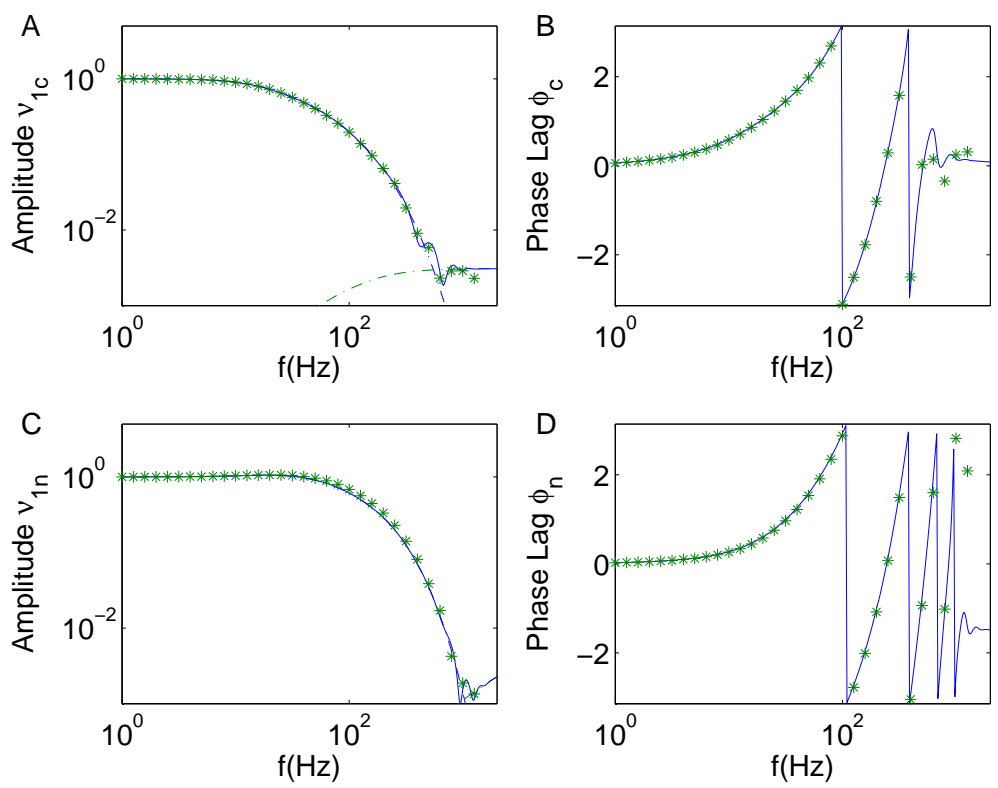

Figure 2.7: The normalized transmission functions $\nu_{1}(\omega) / \nu_{1}(0.1)$ and phase lags for a current coded signal $(\mathrm{A}, \mathrm{B})$ and for a noise coded signal (C,D) with $r=10$ and $\nu_{0}=5 \mathrm{~Hz}$. 

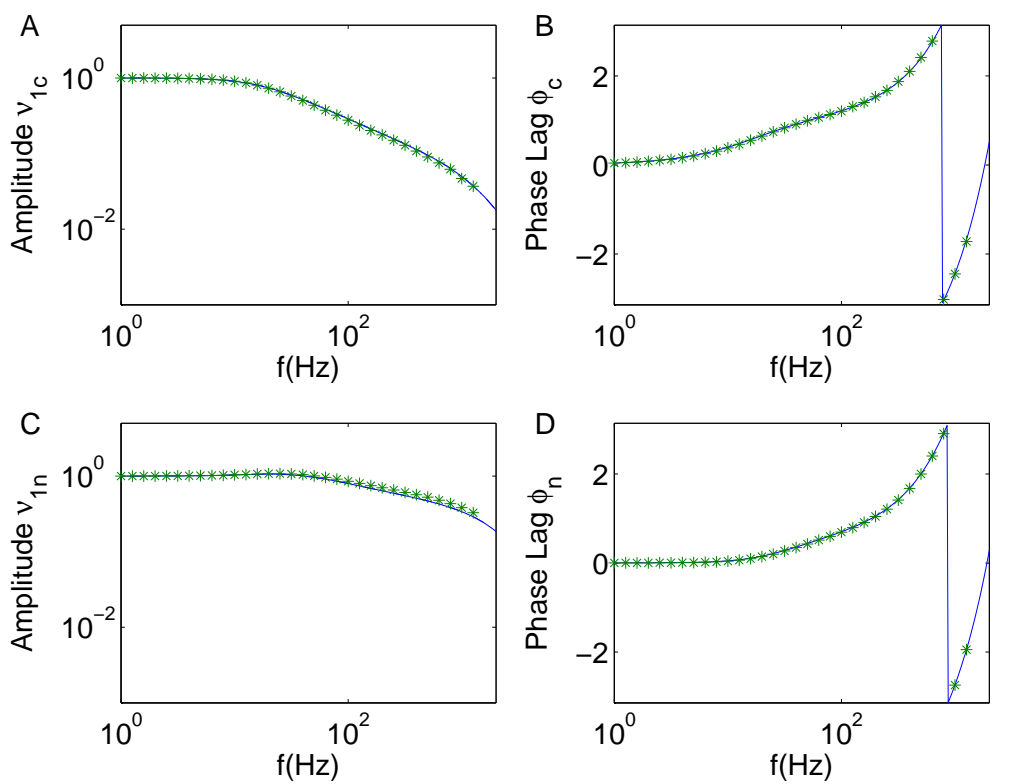

Figure 2.8: The normalized transmission functions $\nu_{1}(\omega) / \nu_{1}(0.1)$ and phase lags for the current coded signal $(A, B)$ and for the noise coded signal $(C, D)$ with $r=100$ and $\nu_{0}=5 \mathrm{~Hz}$.

\subsubsection{Relation to the LIF model}

\section{Large $r$ limit}

In the large $r$ limit, the $r-\tau_{m}$ model has the same dynamics as the LIF model and one can therefore expect them to possess the same dynamical response. We have proven this relation for the stationary firing rate (2.16). Now we will show that for linear response this also holds: the $\nu_{1}^{\text {Low }}(\omega)$ parts of the linear response give exactly the results of the LIF model, while the $\nu_{1}^{\text {High }}(\omega)$ parts decay to zero. When $r \rightarrow \infty, P_{01}\left(v_{0}\right) \rightarrow 0$ and $P_{01}^{\prime}\left(v_{0}\right) \rightarrow-\nu_{0} / D$. Some of the parabolic cylinder functions in the formulas for the linear response have zero or infinity arguments. By applying corresponding formula in the appendix to them, we find in particular that $Y_{1} e^{\Delta_{1}} \rightarrow 0, Y_{1}^{\prime} e^{\Delta_{1}} \rightarrow 1, Y_{2} e^{\Delta_{1}} \rightarrow 0, Y_{2}^{\prime} e^{\Delta_{1}} \rightarrow 0, Y_{3} e^{\Delta_{1}} \rightarrow 0$ and $Y_{3}^{\prime} e^{\Delta_{1}} \rightarrow 0$ in the large $r$ limit. Substituting these into the expressions of $\nu_{1 c}(\omega)$ and $\nu_{1 n}(\omega)$, we find that in the large $r$ limit, the $\nu_{1}^{\text {High }}(\omega)$ parts decay to zero and

$$
\begin{aligned}
& \nu_{1 c}^{\text {Low }}(\omega) \rightarrow \frac{\nu_{0}}{\sqrt{D}} \frac{i \omega}{i \omega-1} \frac{\Phi_{1}\left(v_{0}\right)-\Phi_{1}\left(v_{r}\right) e^{\Delta_{0}}}{\psi_{1}\left(v_{0}\right)-\psi_{1}\left(v_{r}\right) e^{\Delta_{0}+i \omega \tau_{r}}}, \\
& \nu_{1 n}^{\text {Low }}(\omega) \rightarrow \frac{\nu_{0}}{D} \frac{i \omega(i \omega-1)}{2-i \omega} \frac{\Upsilon_{1}\left(v_{0}\right)-\Upsilon_{1}\left(v_{r}\right) e^{\Delta_{0}}}{\psi_{1}\left(v_{0}\right)-\psi_{1}\left(v_{r}\right) e^{\Delta_{0}+i \omega \tau_{r}}},
\end{aligned}
$$

which are exactly the linear response of the LIF model with the threshold at $v_{0}$ (Lindner \& Schimansky-Geier, 2001). 


\section{Large $\omega$ limit}

In the high signal frequency limit, the linear response is shaped exclusively by the absorbing boundary at $v_{b}$ and therefore is expected to be similar for every integrate-and-fire model with finite $v_{b}$. As we will show later, taking a large $v_{b}$ can suppress the boundary induced artifacts. Note that in models with the boundary at infinity, e.g., in QIF and EIF models, the $v_{b} \rightarrow \infty$ limit is taken by default and therefore, the boundary induced artifacts are suppressed. In our model, however, we are allowed to take only a large but finite $v_{b}$ due to the linear dynamics. So if we fix $v_{b}$ and apply the large $\omega$ expansion as given in the appendix to all the parabolic cylinder functions, the high frequency limit of the linear response can be obtained: $\nu_{1 c}(\omega) \rightarrow \frac{\nu_{0}}{\sqrt{D}} \frac{1}{\sqrt{\omega}} e^{i \pi / 4}$ and $\nu_{1 n}(\omega) \rightarrow \frac{\nu_{0}}{D}$. These are provided solely by $\nu_{1}^{\text {High }}(\omega)$, while the $\nu_{1}^{\text {Low }}(\omega)$ parts decay to zero exponentially. This demonstrates that the linear response of $r-\tau_{m}$ model possesses the same high frequency limit as the LIF model, originating from the reset assumption at the truncation point $v_{b}$ of the AP waveform.

\subsubsection{Step response}

The linear response for a small step change $\varepsilon \Theta(t)$, where $\Theta(t)$ is the Heaviside step function, on the mean input current and on the noise level can be calculated using the transfer function we have obtained,

$$
\begin{aligned}
& \nu_{c}(t)=\varepsilon \frac{1}{2 \pi} \int_{-\infty}^{+\infty} H(\omega) \nu_{1 c}^{*}(\omega) e^{i \omega t} d \omega \\
& \nu_{n}(t)=\varepsilon \frac{\sigma}{2 \pi} \int_{-\infty}^{+\infty} H(\omega) \nu_{1 n}^{*}(\omega) e^{i \omega t} d \omega
\end{aligned}
$$

Here the asterisks represent the complex conjugate and $H(\omega)=\pi \delta(\omega)-i / \omega$ is the Fourier transform of the Heaviside step function. In Fig. 2.9 we compare the theoretical results with numerical simulations for a step change in the mean input and in the noise level. The inverse Fourier transform in Eq. (2.56) are evaluated using Matlab. We see that the response is much faster for a noise coded signal than for a mean current coded signal, which is suggested by experiments (Silberberg et al., 2004). The larger is $r$, the faster is the response speed of a population of model neurons to a step change. Note that in the LIF model, the onset rapidness is infinite large and the response function for a noise coded signal keeps a constant value in the large $\omega$ limit, therefore can be taken out of the integral leading to a step change in the instantaneous firing rate immediately. For a step change in the mean input, the response is much slower and there is a much weaker dependence on $r$ as compared with a step change in the noise amplitude. Note that for the theoretical results there are only negligible differences between using $\nu_{1}(\omega)$ and $\nu_{1}^{\text {Low }}(\omega)$. 
One usually used argument for the faster response to a change in the noise level goes as follows: assuming the probability density is insensitive to a fast change in the stimulus, then the change of the firing rate is directly proportional to the change in the noise level and therefore an instantaneous response from the definition of instantaneous firing rate,

$$
\nu(t)=-\frac{1}{2} \sigma^{2} \partial_{v} P_{2}\left(v_{b}, t\right)
$$

But by solving the linear response analytically, we find this argument is valid only for the LIF model. As given in Eq. (2.56), the response to a step change is determined by the response function $\nu_{1 n}(\omega)$ which determines the response to each frequency component in the stimulus. From Section 2.3 .2 ,

$$
\begin{aligned}
\nu_{1 n}(\omega) & =\frac{\nu_{0}}{D}-D q_{2}^{\prime}\left(v_{b}\right) e^{\left(r v_{b}^{2}-2\left(r v_{t}-\mu\right) v_{b}\right) / 4 D} \\
& =\frac{\nu_{0}}{D}-\left(\frac{\nu_{0}}{D}-\nu_{1 n}^{\text {Low }}(\omega)-\nu_{1 n}^{H i g h}(\omega)\right) .
\end{aligned}
$$

The first term is from the change in the noise level directly, while the three terms in the bracket is from the change in the probability density. The above mentioned intuitive argument takes into account only the first term. As will be shown in next section, the $\nu_{1 n}^{\text {High }}(\omega)$ part is suppressed by both a large onset rapidness $r$ and a large boundary value $v_{b}$, and represents the boundary induced artifact. In the $r \rightarrow \infty$ limit, the $\nu_{1 n}^{H i g h}(\omega)$ part is zero from the infinite large $r$ and the $\nu_{1 n}^{\text {Low }}(\omega)$ part has a high frequency limit $\frac{\nu_{0}}{D}$, which makes the contribution from the change of the probability density (terms in the bracket) negligible. When $r$ is finite, however, the first term is concealed by a term from the changing in the probability density and the response is determined by the properties of the physiologically relevant term $\nu_{1 n}^{\text {Low }}(\omega)$. Since $\nu_{1 n}^{\text {Low }}(\omega)$ decays to zero at high frequency, there is no instantaneous component in the step response.

\subsection{Large $v_{b}$ limit and separation of boundary in- duced artifacts}

\subsubsection{Large $v_{b}$ limit}

When the absorbing boundary is far from the unstable fixed point, i.e., $v_{b} \gg v_{t}$, the large argument expansion can be applied to the parabolic cylinder functions with arguments at $v_{b}$. The expressions for the linear response are significantly 

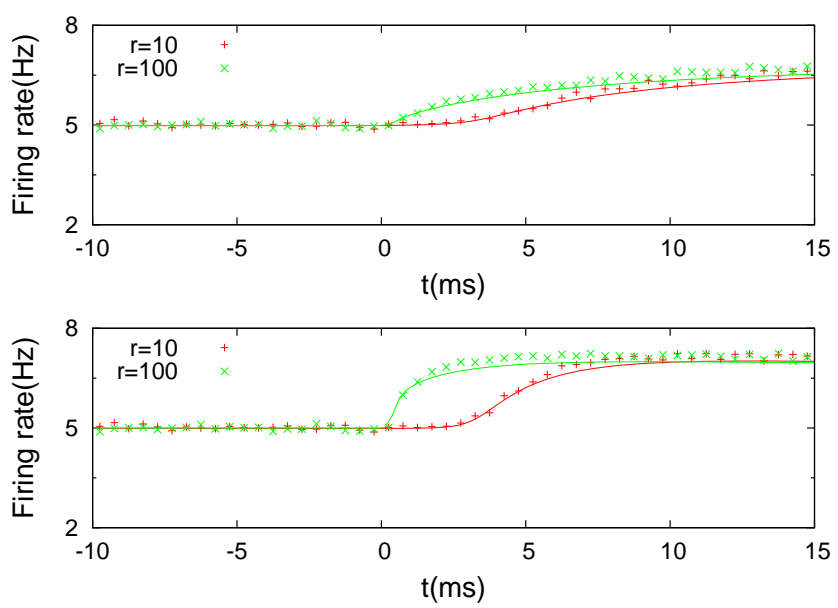

Figure 2.9: The instantaneous firing rate for a step change in the mean input(upper panel) and noise amplitude(lower panel) at $t=0$ for $r=10,100$. $\nu(t)$ changes from $5 \mathrm{~Hz}$ to $7 \mathrm{~Hz}$ in both cases. The solid lines are theoretical results from the linear response, Eq. (2.56). Note that the contributions from $\nu_{1}^{\text {High }}(\omega)$ are negligible. The differences between simulation and theory are from higher order contributions.

simplified and the dependence on $v_{b}$ becomes transparent. First note that

$$
\begin{aligned}
Y_{1}\left(v_{0}\right) e^{\Delta_{1}} \simeq & \sqrt{D / r}\left(\psi_{3}\left(v_{0}\right) \cdot \sin \pi(-i \omega / r+1 / 2)\right. \\
& \left.-\frac{2 \pi}{D / r} \frac{1}{\Gamma(-i \omega / r+1)} \psi_{4}\left(v_{0}\right)\right) e^{x_{1}^{2} / 4-i \omega / r \ln x} \\
= & -\sqrt{D / r} U\left(-i \omega / r+\frac{1}{2}, \frac{r\left(v_{0}-v_{t}\right)+\mu}{\sqrt{r D}}\right) e^{x_{1}^{2} / 4-i \omega / r \ln x} \\
= & -\sqrt{D / r} \phi_{12}\left(v_{0}\right) e^{x_{1}^{2} / 4-i \omega / r \ln x}
\end{aligned}
$$

where $x=\frac{r\left(v_{b}-v_{t}\right)+\mu}{\sqrt{r D}}, x_{1}=\frac{v_{0}-\mu}{\sqrt{r D}}, \phi_{12}(v) \equiv U\left(-i \omega / r+\frac{1}{2}, \frac{r\left(v-v_{t}\right)+\mu}{\sqrt{r D}}\right)$ and we have used Eq. (2.88) in the appendix. Note that $\phi_{12}(v)$ satisfies the same parabolic cylinder equation as $\psi_{3}(v)$ and $\psi_{4}(v)$. Similarly, we have

$$
\begin{aligned}
& Y_{2}\left(v_{0}\right) e^{\Delta_{1}}=-\sqrt{D / r} i \omega / r \phi_{12}\left(v_{0}\right) e^{x_{1}^{2} / 4-i \omega / r \ln x} \cdot \frac{1}{x} \\
& Y_{3}\left(v_{0}\right) e^{\Delta_{1}}=\sqrt{D / r} i \omega(1+i \omega / r) \phi_{12}\left(v_{0}\right) e^{x_{1}^{2} / 4-i \omega / r \ln x} \cdot \frac{1}{x^{2}} .
\end{aligned}
$$


The linear response becomes,

$$
\begin{aligned}
\nu_{1 c}^{\text {Low }}(\omega)= & \frac{\nu_{0}}{\sqrt{D}} \frac{i \omega}{(1-i \omega)(1+i \omega / r)} \times \\
& \frac{(1+1 / r)\left(\psi_{1} \tilde{P}_{01}-\sqrt{D} \Phi_{1} \tilde{P}_{01}^{\prime}\right)-(1+i \omega / r) \Phi_{1}\left(v_{r}\right) e^{\Delta_{0}}}{\psi_{1}\left(v_{r}\right) e^{\Delta_{0}+i \omega \tau_{r}}-\sqrt{D / r}\left(\phi_{12} \psi_{1}^{\prime}-\phi_{12}^{\prime} \psi_{1}\right) e^{x_{1}^{2} / 4-i \omega / r \ln x}}, \\
\nu_{1 c}^{\text {High }}(\omega)= & \frac{\nu_{0}}{r\left(v_{b}-v_{t}\right)+\mu} \frac{-i \omega / r}{1+i \omega / r} \times \\
& \frac{\sqrt{D / r}\left(\phi_{12} \psi_{1}^{\prime}+r \phi_{12}^{\prime} \psi_{1}\right) e^{x_{1}^{2} / 4-i \omega / r \ln x}}{\psi_{1}\left(v_{r}\right) e^{\Delta_{0}+i \omega \tau_{r}}-\sqrt{D / r}\left(\phi_{12} \psi_{1}^{\prime}-\phi_{12}^{\prime} \psi_{1}\right) e^{x_{1}^{2} / 4-i \omega / r \ln x}} .
\end{aligned}
$$

for a current coded signal and

$$
\begin{aligned}
\nu_{1 n}^{\text {Low }}(\omega)= & \frac{\nu_{0}}{D} \frac{i \omega(i \omega-1)}{(2-i \omega)(2+i \omega / r)} \times \\
& \frac{(1+1 / r)\left(\frac{i \omega}{1-i \omega} \Phi_{1} \tilde{P}_{01}+2 \sqrt{D} \Upsilon \tilde{P}_{01}^{\prime}\right)+(2+i \omega / r) \Upsilon\left(v_{r}\right) e^{\Delta_{0}}}{\psi_{1}\left(v_{r}\right) e^{\Delta_{0}+i \omega \tau_{r}}-\sqrt{D / r}\left(\phi_{12} \psi_{1}^{\prime}-\phi_{12}^{\prime} \psi_{1}\right) e^{x_{1}^{2} / 4-i \omega / r \ln x}} \\
\nu_{1 n}^{\text {High }}(\omega)= & \frac{\nu_{0}}{\left(r\left(v_{b}-v_{t}\right)+\mu\right)^{2}} \frac{i \omega(1+i \omega / r)}{2+i \omega / r} \times \\
& \frac{\sqrt{D / r}\left(\phi_{12} \psi_{1}^{\prime}+r \phi_{12}^{\prime} \psi_{1}\right) e^{x_{1}^{2} / 4-i \omega / r \ln x}}{\psi_{1}\left(v_{r}\right) e^{\Delta_{0}+i \omega \tau_{r}}-\sqrt{D / r}\left(\phi_{12} \psi_{1}^{\prime}-\phi_{12}^{\prime} \psi_{1}\right) e^{x_{1}^{2} / 4-i \omega / r \ln x}} \cdot
\end{aligned}
$$

for a noise coded signal.

We see that $\nu_{1}^{\text {High }}(\omega)$ are strongly suppressed when $v_{b}$ is large. The $\nu_{1}^{\text {Low }}(\omega)$ parts, on the contrary, show a much weaker dependence on $v_{b}$, see Fig. 2.10 and 2.11. $\nu_{1}^{\text {Low }}(\omega)$ depend on $v_{b}$ mainly through a frequency dependent phase lag, characterizing the time lag to the truncation point $v_{b}$ of the AP. When $\omega$ is not small $(\omega \geq 1)$, the contribution from $\psi_{1}\left(v_{r}\right)$ term is negligible compared to the other terms in the denominator in the expressions of the linear response. The expressions for $\nu_{1}^{H i g h}(\omega)$ are further simplified,

$$
\begin{aligned}
\nu_{1 c}^{H i g h}(\omega) & \simeq \frac{\nu_{0}}{r\left(v_{b}-v_{t}\right)+\mu} \frac{i \omega}{r+i \omega}, \\
\nu_{1 n}^{H i g h}(\omega) & \simeq-\frac{\nu_{0}}{\left(r\left(v_{b}-v_{t}\right)+\mu\right)^{2}} \frac{i \omega(1+i \omega / r)}{2+i \omega / r} .
\end{aligned}
$$

Above formulas describe the behavior for low and intermediate frequencies, while for even higher signal frequency the large $v_{b}$ approximation is not valid any more. In such a frequency range, the $\nu_{1}^{\text {High }}(\omega)$ parts in the response function will follow the large $\omega$ limit as given in section 2.3.4 and have the same limit behavior as the LIF model. 

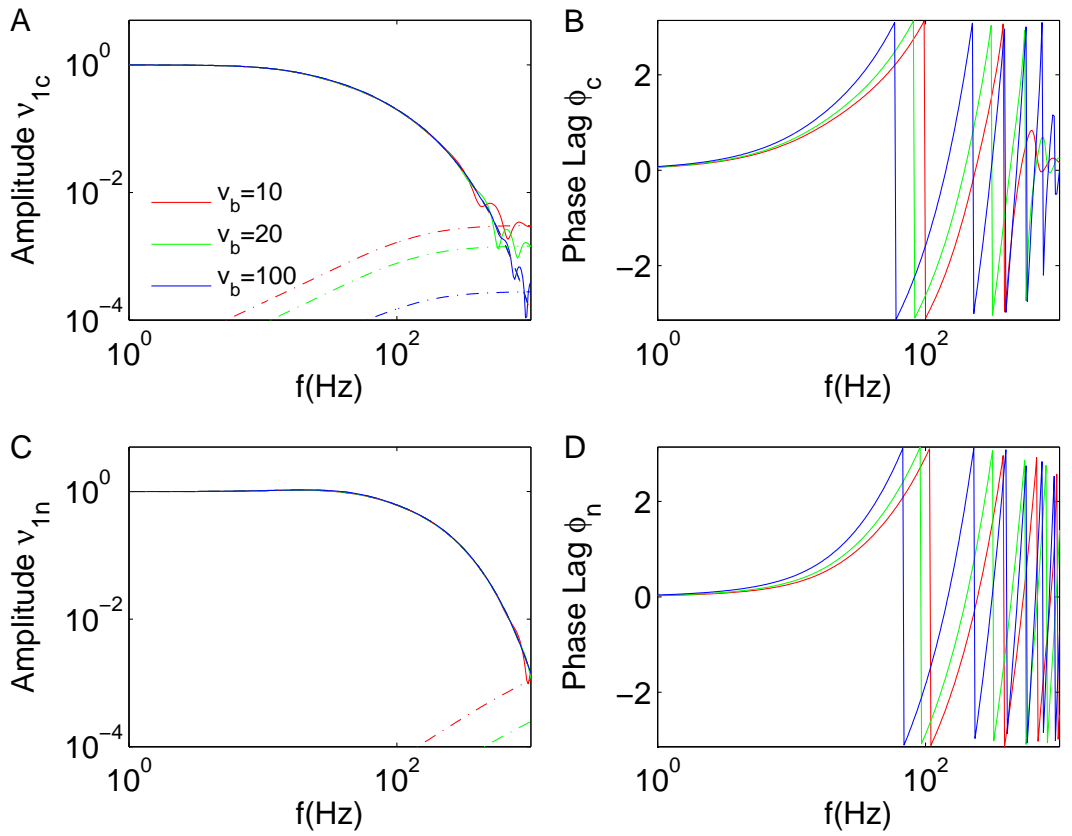

Figure 2.10: Dependence of the linear response on the truncation point $v_{b}$ for a current coded signal (A,B) and for a noise coded signal (C,D) with $r=10$ and $\nu_{0}=5 \mathrm{~Hz}$.
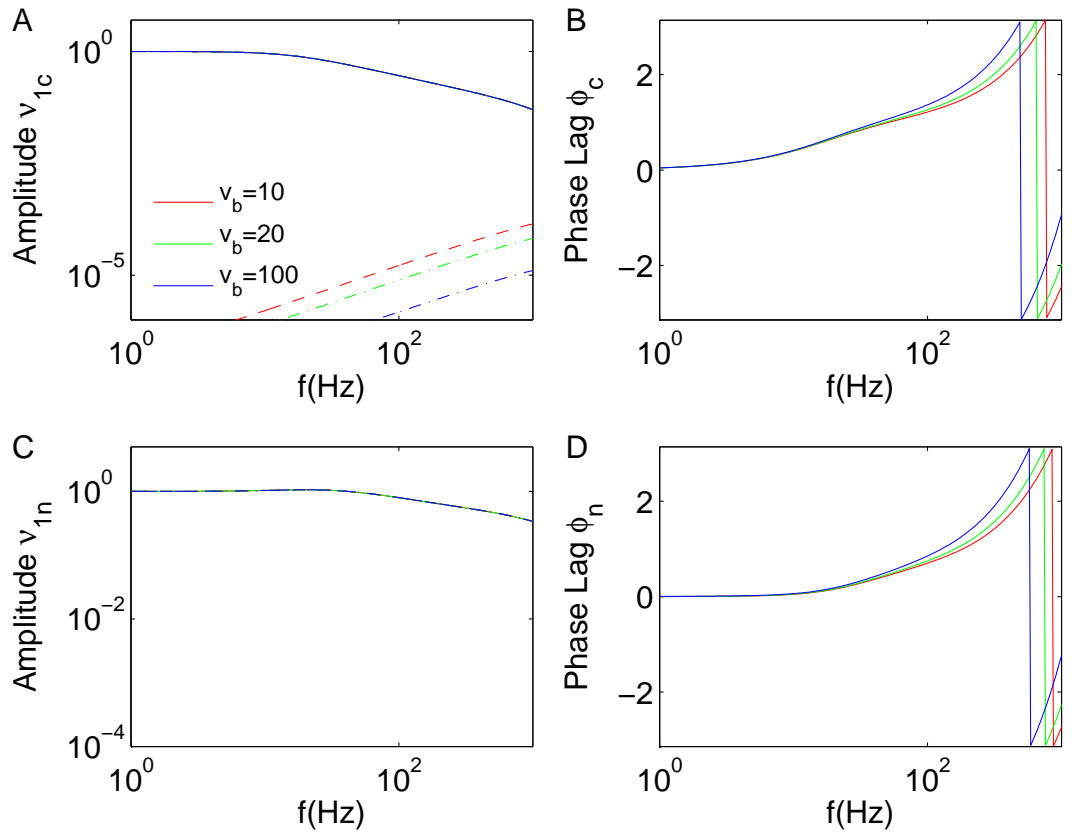

Figure 2.11: Dependence of the linear response on the truncation point $v_{b}$ for a current coded signal (A,B) and for a noise coded signal (C,D) with $r=100$ and $\nu_{0}=5 \mathrm{~Hz}$. 


\subsubsection{Threshold models and boundary induced artifacts}

In an integrate-and-fire (IF) model with linear dynamics, an absorbing boundary at some finite membrane potential $v_{b}$ needs to be assigned. It is known that the absorbing boundary condition at $v_{b}$ can induce severe artifacts in the dynamical response of a population of neurons. The boundary $v_{b}$ marks a "point of no return", which is not present in a biophysical dynamical model of AP initiation. As a consequence, the transmission function for a noise coded signal in the LIF model. for instance. does not decav at high signal frequencies (Silberberg et al., 2004; Lindner \& Schimansky-Geier, 2001). Ideally one would thus wish to separate the response function into a physically meaningful part $\nu_{1}^{\text {phy }}(\omega)$ and a part containing all artifacts such that $\nu_{1}^{\text {phy }}(\omega)=\nu_{1}(\omega)-\nu_{1}^{\text {abs }}(\omega)$. $\nu_{1}^{\text {phy }}(\omega)$ must have the following properties: i) $\nu_{1}^{\text {phy }}(\omega)$ approaches the static susceptibility when $\omega \rightarrow 0$, specifically, $\nu_{1 c}^{p h y}(\omega) \rightarrow \frac{\partial \nu_{0}}{\partial \mu}$ and $\nu_{1 n}^{p h y}(\omega) \rightarrow \frac{1}{\sigma} \frac{\partial \nu_{0}}{\partial \sigma}$; ii) $\nu_{1}^{p h y}(\omega) \rightarrow 0$ when $\omega \rightarrow \infty$; iii) no essential dependence on the truncation point $v_{b}$. The artifactual part from the absorbing boundary should have the following properties: i) negligible contribution for signal frequency in the physiologically relevant range $f \leq 1 \mathrm{kHz}$, i.e., $\left|\nu_{1}^{a b s}(\omega)\right| \ll\left|\nu_{1}^{\text {phy }}(\omega)\right|$, where $f=\omega / 2 \pi$; ii) strong dependence on the truncation point $v_{b}$. We will show that such an isolation of the biophysically meaningful response is possible in our model.

\subsubsection{Separation of boundary induced artifacts}

The decomposing of $\nu_{1}(\omega)$ into two additive components has exactly the features required for the separation of the boundary induced artifacts. From Eq. (2.62), we see $\nu_{1}^{\text {High }}(\omega)$ are strongly dependent on $v_{b}$ and $\nu_{1}^{H i g h}(\omega) \rightarrow 0$ when $v_{b} \rightarrow \infty$, as can also be seen from Fig. 2.10, 2.11 and 2.13 . When $\omega \rightarrow 0, \nu_{1}^{\text {High }}(\omega)$ are negligible compared with $\nu_{1 n}^{\text {Low }}(\omega)$ and are strongly suppressed by increasing of $v_{b}$. That $\nu_{1}^{\text {High }}(\omega)$ capture all artifactual contributions imposed by the absorbing boundary condition is finally confirmed from the high frequency behavior

$$
\begin{aligned}
& \nu_{1 c}^{H i g h}(\omega) \rightarrow \frac{\nu_{0}}{\sqrt{D}} \frac{1}{\sqrt{\omega}} e^{i \pi / 4}=\lim _{\omega \rightarrow \infty} \nu_{1 c}(\omega)=\lim _{\omega \rightarrow \infty} \nu_{1 c}^{L I F}(\omega), \\
& \nu_{1 n}^{H i g h}(\omega) \rightarrow \frac{\nu_{0}}{D}=\lim _{\omega \rightarrow \infty} \nu_{1 n}(\omega)=\lim _{\omega \rightarrow \infty} \nu_{1 n}^{L I F}(\omega),
\end{aligned}
$$

that are identical in every IF model with the absorbing boundary at finite voltage. As a consequence, neglecting the $\nu_{1}^{H i g h}(\omega)$ parts in the response function eliminates any boundary induced instantaneous response components. These results establish that $\nu_{1}^{\text {Low }}(\omega)$ captures the behavior of $\nu_{1}(\omega)$ for low and intermediate frequencies and decays to zero in the high frequency limit. When $\omega \rightarrow 0, \nu_{1 c}^{\text {Low }}(\omega) \rightarrow \nu_{1 c}(0)=\frac{\partial \nu_{0}}{\partial \mu}$ and $\nu_{1 n}^{\text {Low }}(\omega) \rightarrow \nu_{1 n}(0)=\frac{1}{\sigma} \frac{\partial \nu_{0}}{\partial \sigma}$, since $\nu_{1}^{\text {High }}(\omega)$ are negligible there. As shown in Eq. (2.60) and (2.61), $\nu_{1}^{\text {Low }}(\omega)$ also exhibit only a weak dependence on the position of the truncation point. Therefore, we 
have $\nu_{1}^{\text {High }}(\omega)=\nu_{1}^{\text {abs }}(\omega)$ and $\nu_{1}^{\text {Low }}(\omega)=\nu_{1}^{\text {phy }}(\omega)$. The biophysically meaningful predictions of the model can thus be revealed by examining $\nu_{1}^{\text {Low }}(\omega)$ in isolation.

\subsection{Low rate limit}

In the fluctuation driven regime, low rate approximation corresponds to small $\sigma$ limit. For small $\sigma$, the arguments $\frac{v_{0}-\mu}{\sqrt{D}}$ and $\frac{v_{0}-\mu}{\sqrt{r D}}$ are large and the large argument expansion for the parabolic cylinder functions can be applied. By replacing the stationary firing rate with the Kramers rate at the same time, we obtain

$$
\begin{aligned}
& \nu_{1 c}^{\text {Low }}(\omega) \simeq \nu_{0} \frac{v_{0}-\mu}{v_{0} D} \frac{\Gamma(1-i \omega / r)}{(1-i \omega)(1+i \omega / r)} \exp \left(-\frac{i \omega}{r} \log \left(x x_{1}\right)\right), \\
& \nu_{1 n}^{\text {Low }}(\omega) \simeq \nu_{0} \frac{2\left(v_{0}-\mu\right)^{2}}{v_{0} D^{2}} \frac{\Gamma(1-i \omega / r)}{(2-i \omega)(2+i \omega / r)} \exp \left(-\frac{i \omega}{r} \log \left(x x_{1}\right)\right),
\end{aligned}
$$

where $x=\frac{r\left(v_{b}-v_{t}\right)+\mu}{\sqrt{r D}}$ and $x_{1}=\frac{v_{0}-\mu}{\sqrt{r D}}$. Here we have dropped corrections at the order of $\frac{r D \omega^{2}}{\left(v_{0}-\mu\right)^{2}}$ and $\frac{D \omega^{2}}{\left(v_{0}-\mu\right)^{2}}$. This means that the above formulas are valid only for $\omega \ll \frac{v_{0}-\mu}{\sqrt{r D}}$, as can be seen from Fig. 2.12. The $\nu_{1}^{\text {High }}(\omega)$ parts are negligible in this frequency range. The linear responses thus decay as a combination of power law and exponential decay in the low rate limit. When $\mathrm{r}$ is large, the $r-\tau_{m}$ model behaves like a low-pass filter with the cutoff frequency insensitive to $r$. Above formulas are consistent with the adiabatic responses given by $\nu_{1 c}(0)=\frac{\partial \nu_{0}}{\partial \mu}$ and $\nu_{1 n}(0)=\frac{\partial \nu_{0}}{\partial D}$, if the Kramers rate for $\nu_{0}$ is used.

\subsection{High frequency behavior: $r$ and $f_{c}$}

For the biophysically relevant response function $\nu_{1}^{\text {Low }}(\omega)$, we take $v_{b} \gg v_{t}$ and apply the large argument expansion for the parabolic cylinder functions with argument at $v_{b}$ and large $\omega$ expansion for those with arguments at $v_{r}$ and $v_{0}$. We then obtain

$$
\begin{aligned}
\nu_{1 c}^{\text {Low }}(\omega) \simeq & \frac{\nu_{0}}{\sqrt{D}} \frac{-\sqrt{2}\left(1+\frac{1}{r}\right) i \omega \sqrt{i \omega / r-1}}{(1-i \omega)(1+i \omega / r)(\sqrt{i \omega / r}+\sqrt{i \omega / r-1})}\left(\frac{\bar{P}_{01}}{\sqrt{r}}-\frac{i \sqrt{D}}{\sqrt{i \omega-1}} \tilde{P}_{01}^{\prime}\right) \\
& \times \exp \left(-\frac{\pi}{4 r} \omega+i x_{1} \sqrt{i \omega / r-1}-\frac{x_{1}^{2}}{4}+\frac{i \omega}{2 r}(1-\log (\omega / r)+2 \log (x))\right), \\
\nu_{1 n}^{\text {Low }}(\omega) \simeq & \frac{\nu_{0}}{D} \frac{\sqrt{2}\left(1+\frac{1}{r}\right) i \omega \sqrt{i \omega / r-1}}{(2-i \omega)(2+i \omega / r)(\sqrt{i \omega / r}+\sqrt{i \omega / r-1})}\left(2 \sqrt{D} \tilde{P}_{01}^{\prime}+i \sqrt{i \omega / r} \bar{P}_{01}\right) \\
& \times \exp \left(-\frac{\pi}{4 r} \omega+i x_{1} \sqrt{i \omega / r-1}-\frac{x_{1}^{2}}{4}+\frac{i \omega}{2 r}(1-\log (\omega / r)+2 \log (x))\right),
\end{aligned}
$$



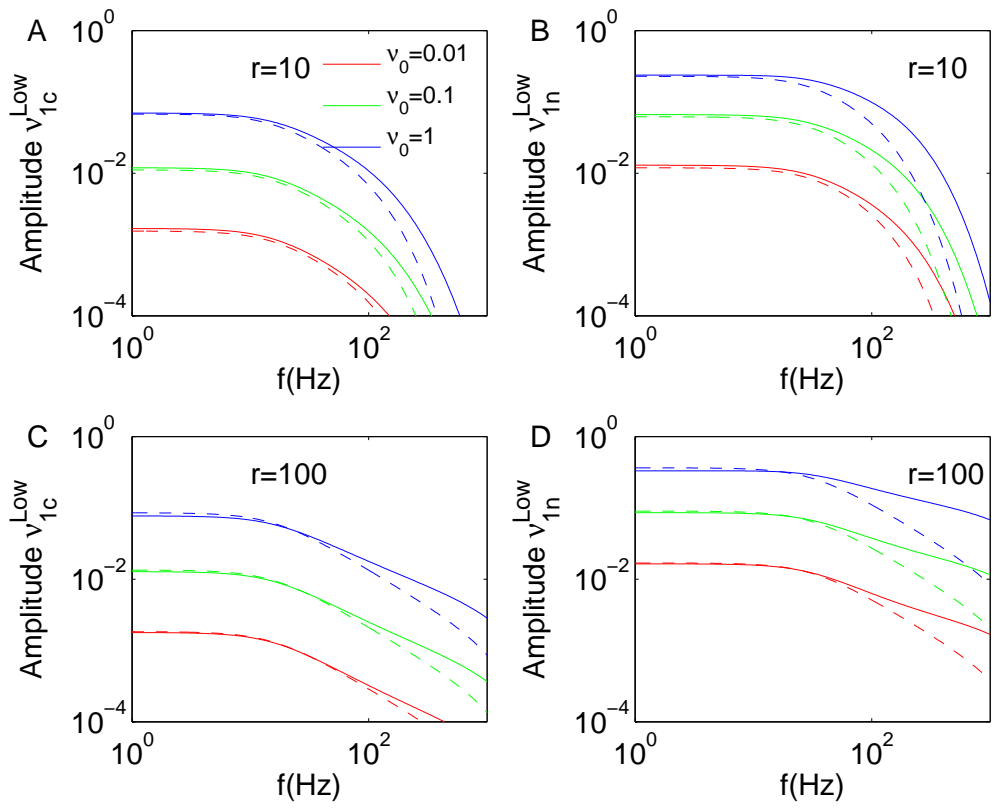

Figure 2.12: The low rate approximation for the linear response with $r=10,100$ and $\nu_{0}=0.01,0.1$ and $1 \mathrm{~Hz}$. A and $\mathrm{C}$ are for a current coded signal; B and D are for a noise coded signal. Solid lines are the accurate results from Eq. (2.40) and (2.51); dashed lines are the low rate approximations from Eq. (2.64).

where $x=\frac{r\left(v_{b}-v_{t}\right)+\mu}{\sqrt{r D}}, x_{1}=\frac{v_{0}-\mu}{\sqrt{r D}}$ and $\tilde{P}_{01}(v)=\sqrt{r} \bar{P}_{01}(v)$. Here corrections at order $\omega^{2} / x^{2}$ and $Q_{1}\left(\frac{v_{0}-\mu}{\sqrt{D}}\right) / \sqrt{\omega}$ are ignored, where $Q_{1}(v)$ is the first correction term for large $\omega$ expansion, see Eq. (2.96) in the appendix. The above formulas give the high frequency behavior of $\nu_{1}^{\text {Low }}(\omega)$ in the biophysical relevant regime. Fig. 2.13 compares the approximations Eq. (2.62) and Eq. (2.65) with the exact values for different $r$. The approximations agree quite well with the exact values for $\nu_{1}^{\text {High }}(\omega)$ for a wide scope of signal frequencies, ranging from quite low to quite high frequencies, and $\nu_{1}^{\text {Low }}(\omega)$ for high signal frequencies.

When $r \rightarrow \infty, \bar{P}_{01}\left(v_{0}\right) \rightarrow \sqrt{\frac{\pi}{2}}$ and $\tilde{P}_{01}^{\prime}\left(v_{0}\right) \rightarrow-\frac{1}{\sqrt{D}}$, so $\left|\frac{\bar{P}_{01}\left(v_{0}\right)}{\sqrt{D} \tilde{P}_{01}^{\prime}\left(v_{0}\right)}\right| \rightarrow \sqrt{\frac{\pi}{2}}$. As a result, for large $\omega, \nu_{1 n}^{\text {Low }}(\omega)$ decays as

$$
\nu_{1 n}^{\text {Low }}(\omega) \propto \frac{1}{\sqrt{\omega / r}} \exp \left(-\frac{\pi}{4} \omega / r\right) .
$$

depending essentially only on $\omega / r$ and the cutoff frequencies are determined by the time constant $\tau_{m} / r$ for the AP initiation. $\nu_{1 c}^{\text {Low }}(\omega)$ behaves a bit differently for small and large $r$, with

$$
\nu_{1 c}^{\text {Low }}(\omega) \propto \frac{1}{\omega / r} \exp \left(-\frac{\pi}{4} \omega / r\right)
$$



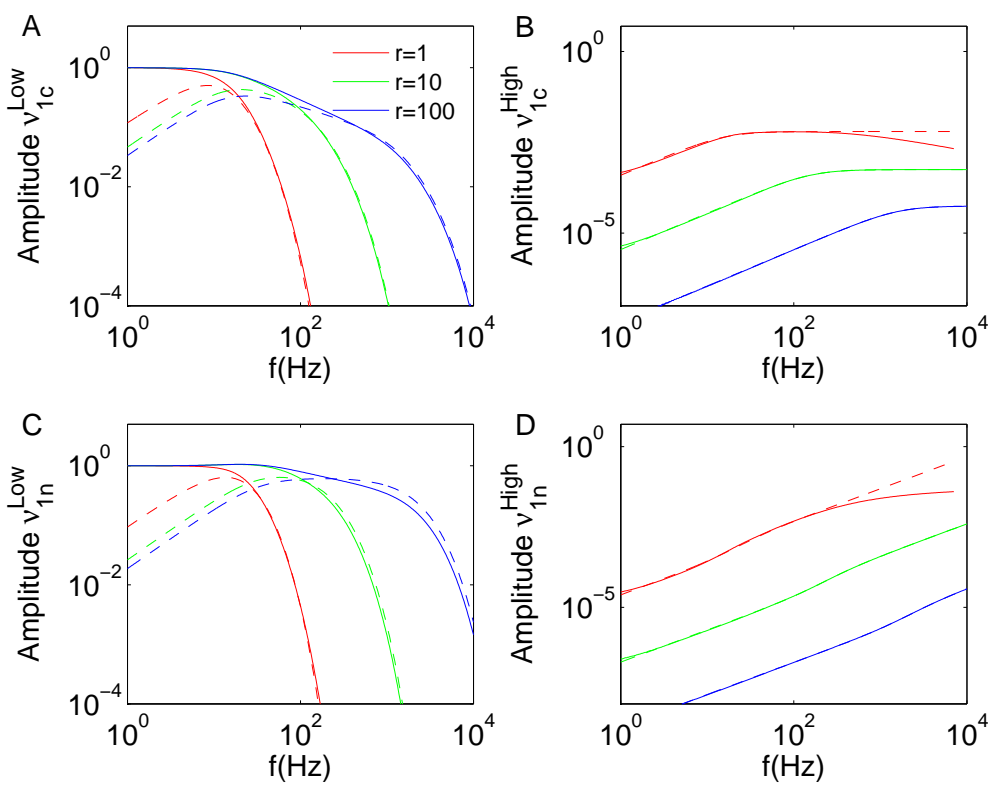

Figure 2.13: The asymptotic approximation Eq. (2.65) for $\nu_{1}^{\text {Low }}(\omega)(\mathrm{A}, \mathrm{C})$ and Eq. (2.62) for $\nu_{1}^{H i g h}(\omega)(\mathrm{B}, \mathrm{D})$ in the linear response with $r=1,10,100$ and $\nu_{0}=5 \mathrm{~Hz}$. For the $\nu_{1}^{\text {Low }}(\omega)$ parts we normalize with the theoretical values at $f=0.1 \mathrm{~Hz}$.

for small $r$, and

$$
\nu_{1 c}^{L o w}(\omega) \propto \frac{1}{\sqrt{\omega}} \exp \left(-\frac{\pi}{4} \omega / r\right)
$$

for large $r$. We see that for a noise coded signal the cut-off frequency is determined solely by the shorter time constant $\tau_{m} / r$, that is, by the AP onset dynamics. So the cutoff frequency $f_{c}$ for a noise coded signal follows $f_{c} \propto r$ and dissociates from $\tau_{m}$. In the LIF model, $r$ is infinite large, so the response function is flat and no attenuation at high frequency occurs for a noise coded signal. Note that a linear relationship $f_{c} \propto r$ was previously conjectured by Naundorf et al. (2007) based on dimensional analysis. Fig. 2.14 shows the behavior of $\nu_{1}^{\text {Low }}(\omega)$ with increasing $r$ and how the cutoff frequency $f_{c}$ changes with $\mathrm{r}$ for different $\nu_{0}$. For the LIF model, $\left|\nu_{1 n}(\omega)\right|$ starts at zero signal frequency with a value $\frac{\partial \nu_{0}}{\partial D}$ and saturates to a constant $\frac{\nu_{0}}{D}$ at large frequency. So in our model there will be a transition between these two plateaus before the decaying of transmission function, since in large $r$ limit it reduces to the LIF model. To characterize the decaying property and avoid the transition region, we extract the cutoff frequencies at which the normalized transmission function decays to $1 / \sqrt{10}$, which is smaller than the value usually adopted. The increasing of $f_{c}$ with the firing rate $\nu_{0}$ is from the stochastic double resonance phenomenon: the transmission function will develop a peak for some optimal signal frequency before decaying when $\nu_{0}$ is larger than 

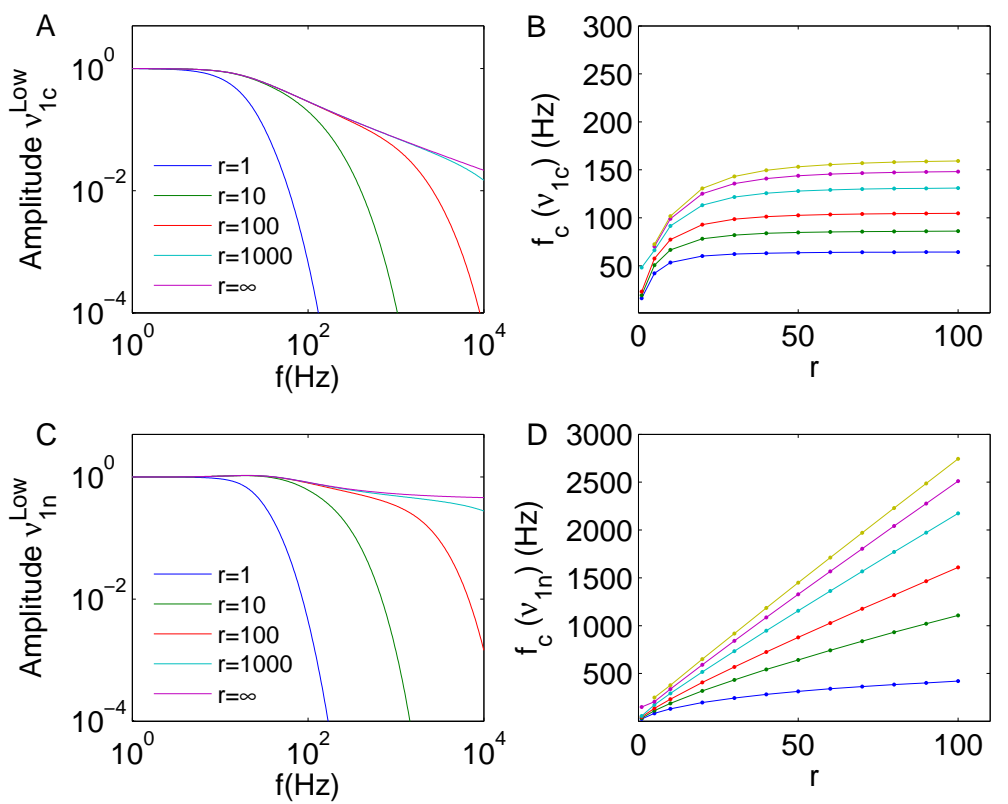

Figure 2.14: Left, the normalized transmission function $\nu_{1}^{\text {Low }}(\omega) / \nu_{1}(0.1)$ for a current coded signal (A) and for a noise coded signal (C) with different r. $\nu_{0}=$ $5 \mathrm{~Hz}$. Right, the variation of the cut-off frequency (B for a current coded signal and $\mathrm{D}$ for a noise coded signal) with the onset rapidness $r$ for different firing rates: $\nu_{0}=1,5,10,20,30,40 \mathrm{~Hz}$ from lower to upper curves. 
some value and behave as a band-pass filter, which can shift the cutoff frequency to a higher value (Plesser \& Geisel, 1999). It can be very large when the onset rapidness $r$ is large and $\nu_{0}$ is not very low $(>1 \mathrm{~Hz}$ here) for a noise coded signal. When the stationary firing rate is low, the cutoff frequency becomes insensitive to $r$. This can be understood from the low firing rate behavior of $\nu_{1 n}^{\text {Low }}(\omega)$. In the low rate limit, we find $\nu_{1 c}^{\text {Low }}(\omega) \propto \frac{1}{1-i \omega}$ and $\nu_{1 n}^{\text {Low }}(\omega) \propto \frac{1}{2-i \omega}$ for large $r$ from Eq. (2.64), therefore the cutoff frequency is insensitive to the onset rapidness $r$ when $r$ is large.

For a current coded signal, on the contrary, the decay of $\nu_{1 c}^{\text {Low }}(\omega)$ is constrained by the membrane time constant and approaching the behavior of the LIF neurons in the large $r$ limit, as shown in Fig. 2.14A. Therefore, fast AP onset can enhance the response speed for a noise coded signal much more significantly than for a current coded signal in the case of white noise. In order to realize a high cutoff frequency for a current coded signal as observed in experiments, other factors, e.g., the correlation time of noise, need to be taken into account.

\section{7 $r=0$ case: a new kind of perfect integrator}

If we let $r=0$ in the $r-\tau_{m}$ model, the evolvement of membrane potential will follow a Brownian motion with a drift term $\mu-v_{0}$ when $v>v_{0}$. In this special case our model resembles the perfect integrator (Gerstein \& Mandelbrot, 1964), also called the simplest integrate-and-fire model (Knight, 1972a), but with a soft boundary at $v=v_{0}$, in the sense that the probability for finding the membrane potential lower than 0 decays as $e^{-(v-\mu)^{2} / \sigma^{2}}$. In the Perfect Integrator model, the neuron performs Brownian motion in the whole range of membrane potential lower than the threshold with the input current as a drift force. The linear response of the model has been obtained (Bulsara et al., 1994: Fourcaud \& Brunel, 2002). One drawback of this model is that the stationary firing rate is zero when the mean input current is zero, no matter how strong is the synapse noise. But the cortical circuits usually sustain in a so called balanced state, where the mean input current to a neuron is near zero from cancelation between excitatory and inhibitory synaptic inputs and the neuron is driven to fire spikes mainly by synaptic noise. When we add a soft barrier here, the neuron can be driven to fire by both the mean input current and the synaptic noise.

The stationary probability density $P_{0}(v)$ and the stationary firing rate can be 
obtained directly by taking $r \rightarrow 0$ in Eq. (2.14) and (2.15),

$$
\begin{aligned}
P_{01}(v)= & \nu_{0} e^{-(v-\mu)^{2} / \sigma^{2}}\left(\frac{2}{\sigma} \int_{\left(\max \left(v, v_{r}\right)-\mu\right) / \sigma}^{\left(v_{0}-\mu\right) / \sigma} e^{y^{2} d y}\right. \\
& \left.+\frac{1}{v_{0}-\mu} e^{\left(\mu^{2}-v_{0}^{2}\right) / \sigma^{2}}\left(e^{\frac{2}{\sigma^{2}}\left(v_{0}-\mu\right) v_{t}}-e^{\frac{2}{\sigma^{2}}\left(v_{0}-\mu\right) v_{0}}\right)\right) \\
P_{02}(v)= & \frac{\nu_{0}}{v_{0}-\mu}\left(e^{\frac{2}{\sigma^{2}}\left(v_{0}-\mu\right)\left(v_{t}-v\right)}-1\right) \\
\nu_{0}^{-1}= & \sqrt{\pi} \int_{\left(\mu-v_{0}\right) / \sigma}^{\left(\mu-v_{r}\right) / \sigma} e^{y^{2}} \operatorname{Erfc}(y) d y \\
& +\frac{\sqrt{\pi}}{2} \frac{\sigma}{v_{0}-\mu} e^{\left(\mu^{2}-v_{0}^{2}\right) / \sigma^{2}} \operatorname{Erfc}\left(\left(\mu-v_{0}\right) / \sigma\right)\left(e^{\frac{2}{\sigma^{2}}\left(v_{0}-\mu\right) v_{t}}-e^{\frac{2}{\sigma^{2}}\left(v_{0}-\mu\right) v_{0}}\right) \\
& +\frac{1}{2} \frac{\sigma^{2}}{\left(v_{0}-\mu\right)^{2}}\left(e^{\frac{2}{\sigma^{2}}\left(v_{0}-\mu\right)\left(v_{t}-v_{0}\right)}-1\right)-\frac{v_{t}-v_{0}}{v_{0}-\mu}
\end{aligned}
$$

For $\mu=v_{0}$, there are

$$
\begin{aligned}
P_{01}(v) & =\frac{\nu_{0}}{D} e^{-v^{2} / \sigma^{2}}\left(\sigma \int_{\left(\max \left(v, v_{r}\right)-\mu\right) / \sigma}^{\left(v_{0}-\mu\right) / \sigma} e^{y^{2} d y}+v_{t}-v_{0}\right) \\
P_{02}(v) & =\frac{\nu_{0}}{D}\left(v_{t}-v\right) \\
\nu_{0}^{-1} & =\sqrt{\pi} \int_{-v_{0} / \sigma}^{-v_{r} / \sigma} e^{y^{2}} \operatorname{Erfc}(y) d y+\sqrt{\pi} \frac{v_{t}-v_{0}}{\sigma}+\frac{\left(v_{t}-v_{0}\right)^{2}}{\sigma^{2}}
\end{aligned}
$$

We see from Eq. (2.70) that the model neuron can be driven to fire by purely synaptic noise, more realistic compared with the perfect integrator model. Note that a reflecting boundary at the resting potential was introduced in (Fusi \& Mattia, 1999).

To obtained the linear response for $r=0$, the following replacements for the parabolic cylinder functions should be made,

$$
\psi_{3}(v)=e^{a v}, \quad \psi_{4}(v)=\frac{1}{2 a} e^{-a v}
$$

where $a=\sqrt{\frac{\left(\mu-v_{0}\right)^{2}}{4 D^{2}}-\frac{i \omega}{D}}$. They are solutions of the homogeneous parts of the FPE (2.30) and (2.44) when $r=0$ and satisfy the same normalization condition, $\psi_{3}^{\prime}(v) \psi_{4}(v)-\psi_{3}(v) \psi_{4}^{\prime}(v)=1$. Correspondingly, according to the recurrence 
relation satisfied by the parabolic cylinder function, we obtain

$$
\begin{aligned}
\Phi_{3}(v) & =\sqrt{\frac{D}{r}}\left(\psi_{3}^{\prime}(v)-\frac{\mu-v_{0}}{2 D} \psi_{3}(v)\right)=\sqrt{\frac{D}{r}}\left(a-\frac{\mu-v_{0}}{2 D}\right) e^{a v} \\
\Upsilon_{3}(v) & =\sqrt{\frac{D}{r}}\left(\Phi_{3}^{\prime}(v)-\frac{\mu-v_{0}}{2 D} \Phi_{3}(v)\right)=\frac{D}{r}\left(a-\frac{\mu-v_{0}}{2 D}\right)^{2} e^{a v} \\
\Phi_{4}(v) & =\frac{\sqrt{r D}}{i \omega}\left(\psi_{4}^{\prime}(v)-\frac{\mu-v_{0}}{2 D} \psi_{4}(v)\right)=-\frac{\sqrt{r D}}{i \omega}\left(a+\frac{\mu-v_{0}}{2 D}\right) \frac{1}{2 a} e^{-a v} \\
\Upsilon_{4}(v) & =\frac{\sqrt{r D}}{i \omega}\left(\Phi_{4}^{\prime}(v)-\frac{\mu-v_{0}}{2 D} \Phi_{4}(v)\right)=\frac{r D}{(i \omega)^{2}}\left(a+\frac{\mu-v_{0}}{2 D}\right)^{2} \frac{1}{2 a} e^{-a v}
\end{aligned}
$$

and

$$
\begin{aligned}
Y_{1}(v) & =\psi_{3}(v) \psi_{4}\left(v_{b}\right)-\psi_{3}\left(v_{b}\right) \psi_{4}(v) \\
& =\frac{1}{2 a}\left(e^{a\left(v-v_{b}\right)}-e^{a\left(v_{b}-v\right)}\right) \\
Y_{2}(v) & =\psi_{4}(v) \Phi_{3}\left(v_{b}\right)-\frac{i \omega}{r} \psi_{3}(v) \Phi_{4}\left(v_{b}\right) \\
& =\frac{1}{2 a} \sqrt{\frac{D}{r}}\left(\left(a-\frac{\mu-v_{0}}{2 D}\right) e^{a\left(v_{b}-v\right)}+\left(a+\frac{\mu-v_{0}}{2 D}\right) e^{a\left(v-v_{b}\right)}\right) \\
Y_{3}(v) & =\psi_{4}(v) \Upsilon_{3}\left(v_{b}\right)-\frac{i \omega}{r}\left(1+\frac{i \omega}{r}\right) \psi_{3}(v) \Upsilon_{4}\left(v_{b}\right) \\
& =\frac{1}{2 a} \frac{D}{r}\left(\left(a-\frac{\mu-v_{0}}{2 D}\right)^{2} e^{a\left(v_{b}-v\right)}-\left(a+\frac{\mu-v_{0}}{2 D}\right)^{2} e^{a\left(v-v_{b}\right)}\right)
\end{aligned}
$$

Substituting these functions into the linear response for positive $r$, Eq. (2.40) and (2.51), we get the results for $r=0$ case,

$$
\begin{aligned}
\nu_{1 c}= & \left(\psi_{1}\left(v_{r}\right) e^{\Delta_{0}}-\left(\frac{1}{a} \psi_{1}^{\prime} \sinh a\left(v_{t}-v_{0}\right)+\psi_{1} \cosh a\left(v_{t}-v_{0}\right) e^{\Delta_{1}}\right)^{-1}\right. \\
& \times\left(\frac{1}{1-i \omega}\left(\psi_{1} P_{02}-\sqrt{D} \phi_{1} P_{02}^{\prime}-i \omega \phi_{1}\left(v_{r}\right) e^{\Delta_{0}}\right)\right. \\
& \left.-\frac{\nu_{0}}{D}\left(\frac{1}{a} K_{1} \psi_{1}^{\prime}+K_{2} \psi_{1}\right) e^{\Delta_{1}}\right) \\
\nu_{1 n}= & \left(\psi_{1}\left(v_{r}\right) e^{\Delta_{0}}-\left(\frac{1}{a} \psi_{1}^{\prime} \sinh a\left(v_{t}-v_{0}\right)+\psi_{1} \cosh a\left(v_{t}-v_{0}\right)\right) e^{\Delta_{1}}\right)^{-1} \\
& \times\left(\frac{i \omega-1}{2-i \omega}\left(\frac{i \omega}{1-i \omega} \phi_{1} P_{02}+2 \sqrt{D} \Upsilon_{1} P_{02}^{\prime}+i \omega \Upsilon_{1}\left(v_{r}\right) e^{\Delta_{0}}\right)\right. \\
& \left.+\frac{\nu_{0}}{D}\left(\frac{1}{a} K_{3} \psi_{1}^{\prime}+K_{4} \psi_{1}\right) e^{\Delta_{1}}\right)
\end{aligned}
$$


where

$$
\begin{aligned}
& K_{1}=\frac{1}{2}\left(\frac{1}{a+\frac{\mu-v_{0}}{2 D}} e^{a\left(v_{b}-v_{0}\right)}+\frac{1}{a-\frac{\mu-v_{0}}{2 D}} e^{a\left(v_{0}-v_{b}\right)}\right), \\
& K_{2}=\frac{1}{2}\left(\frac{1}{a+\frac{\mu-v_{0}}{2 D}} e^{a\left(v_{b}-v_{0}\right)}-\frac{1}{a-\frac{\mu-v_{0}}{2 D}} e^{a\left(v_{0}-v_{b}\right)}\right), \\
& K_{3}=\frac{1}{2}\left(\frac{a-\frac{\mu-v_{0}}{2 D}}{a+\frac{\mu-v_{0}}{2 D}} e^{a\left(v_{b}-v_{0}\right)}-\frac{a+\frac{\mu-v_{0}}{2 D}}{a-\frac{\mu-v_{0}}{2 D}} e^{a\left(v_{0}-v_{b}\right)}\right), \\
& K_{4}=\frac{1}{2}\left(\frac{a-\frac{\mu-v_{0}}{2 D}}{a+\frac{\mu-v_{0}}{2 D}} e^{a\left(v_{b}-v_{0}\right)}+\frac{a+\frac{\mu-v_{0}}{2 D}}{a-\frac{\mu-v_{0}}{2 D}} e^{a\left(v_{0}-v_{b}\right)}\right),
\end{aligned}
$$

and $\Delta_{0}$ and $\Delta_{1}$ are defined in Eq. (2.35). In the $r \rightarrow 0$ limit, $\Delta_{1}$ is also simplified: $\Delta_{1}=\frac{1}{2 D}\left(\mu-v_{0}\right)\left(v_{b}-v_{0}\right)$.

For the perfect integrator, there is a complementary relation between $\nu_{1 c}$ and $\nu_{1 n}$ (Pressley \& Trover, 2009), which is not held any more when the leak current is taken into account.

Note that the above results have a default assumption, $v_{r}<v_{0}$. This is reasonable for $r>0$ when we study the influence of AP onset on dynamical response. But now there is no spiking generating membrane current, $v_{r}$ can be adopted at a value larger than $v_{0}$. Here we provide also the results for this case. For simplicity we take $v_{0}=0$ without losing generality.

When $v_{r}>0$, the stationary solutions of the FPE (2.3) with $r=0$ satisfy

$$
\begin{array}{lr}
\left(-v+\mu-\frac{1}{2} \sigma^{2} \partial_{v}\right) P_{01}(v)=0, & -\infty<v \leq 0 \\
\left(\mu-\frac{1}{2} \sigma^{2} \partial_{v}\right) P_{02}(v)=0, & 0<v \leq v_{r} \\
\left(\mu-\frac{1}{2} \sigma^{2} \partial_{v}\right) P_{02}(v)=\nu_{0}, & v_{r}<v \leq v_{t}
\end{array}
$$

The stationary probability density $P_{01}(v)$ and $P_{02}(v)$ are easy to find,

$$
\begin{aligned}
P_{01}= & \frac{\nu_{0}}{\mu} e^{-\frac{1}{\sigma^{2}}\left(v^{2}-2 \mu v\right)}\left(e^{-\frac{\mu}{D} v_{r}}-e^{-\frac{\mu}{D} v_{t}}\right), \\
P_{02}= & \frac{\nu_{0}}{\mu}\left(e^{\frac{\mu}{D}\left(v-\max \left(v, v_{r}\right)\right.}-e^{\frac{\mu}{D}\left(v-v_{t}\right.}\right), \\
\nu_{0}^{-1}= & \frac{\sqrt{\pi}}{2} \frac{\sigma}{\mu}\left(e^{-\frac{\mu}{D} v_{r}}-e^{-\frac{\mu}{D} v_{t}}\right) e^{\mu^{2} / \sigma^{2}} \operatorname{Erfc}(\mu / \sigma) \\
& \left.+\frac{1}{\mu}\left(v_{t}-v_{r}\right)-\frac{\sigma^{2}}{2 \mu^{2}}\left(e^{-\frac{\mu}{D} v_{r}}-e^{-\frac{\mu}{D} v_{t}}\right)\right) .
\end{aligned}
$$


For $\mu=0$, there are

$$
\begin{aligned}
P_{01}(v) & =\frac{\nu_{0}}{D} e^{-v^{2} / \sigma^{2}}\left(v_{t}-v_{r}\right) \\
P_{02}(v) & =\frac{\nu_{0}}{D}\left(v_{t}-\max \left(v, v_{r}\right)\right) \\
\nu_{0}^{-1} & =\frac{\sqrt{\pi}}{\sigma}\left(v_{t}-v_{r}\right)+\frac{1}{\sigma^{2}}\left(v_{t}^{2}-v_{r}^{2}\right) .
\end{aligned}
$$

The linear response for current and noise coded signal are

$$
\begin{aligned}
\nu_{1 c}= & \frac{\nu_{0}}{D}\left(\psi_{1}(0)\left(e^{-\frac{\mu v_{r}}{2 D}} \cosh a v_{r}-e^{-\frac{\mu v_{t}}{2 D}} \cosh a v_{t}\right)\right. \\
& \left.+\frac{1}{a} \psi_{1}^{\prime}(0)\left(e^{-\frac{\mu v_{r}}{2 D}} \sinh a v_{r}-e^{-\frac{\mu v_{t}}{2 D}} \sinh a v_{t}\right)\right)^{-1} \\
& \left.\times\left(\frac{D}{\mu}\left(e^{-\frac{\mu}{D} v_{r}}-e^{-\frac{\mu}{D} v_{t}}\right) \Upsilon_{1}(0)+\frac{K_{5}}{a} \psi_{1}^{\prime}(0)+K_{6} \psi_{1}(0)\right)\right) \\
\nu_{1 n}= & \frac{\nu_{0}}{D}\left(\psi_{1}(0)\left(e^{-\frac{\mu v_{r}}{2 D}} \cosh a v_{r}-e^{-\frac{\mu v_{t}}{2 D}} \cosh a v_{t}\right)\right. \\
& \left.+\frac{1}{a} \psi_{1}^{\prime}(0)\left(e^{-\frac{\mu v_{r}}{2 D}} \sinh a v_{r}-e^{-\frac{\mu v_{t}}{2 D}} \sinh a v_{t}\right)\right)^{-1} \\
& \times\left(\frac{1}{a} \psi_{1}^{\prime}(0)\left(\left(\sinh a v_{r}-\sinh a v_{t}\right) e^{-\frac{\mu v_{t}}{2 D}}-\frac{\mu}{D} K_{5}\right)\right. \\
& +\psi_{1}(0)\left(\left(\cosh a v_{r}-\cosh a v_{t}\right) e^{-\frac{\mu v_{t}}{2 D}}-\frac{\mu}{D} K_{6}\right) \\
& \left.+\frac{1}{2-i \omega}\left(-i \omega \frac{\sqrt{D}}{\mu} \phi_{1}(0)+2(i \omega-1) \Upsilon_{1}(0)\right)\left(e^{-\frac{\mu}{D} v_{r}}-e^{-\frac{\mu}{D} v_{t}}\right)\right)
\end{aligned}
$$

where $a=\sqrt{\frac{\mu^{2}}{4 D^{2}}-\frac{i \omega}{D}}, \psi_{1}(v), \phi_{1}(v)$ and $\Upsilon_{1}(v)$ are parabolic cylinder functions defined in the appendix, and

$$
\begin{aligned}
& K_{5}=\frac{1}{2}\left(\frac{1}{a+\frac{\mu}{2 D}}\left(e^{\left(a-\frac{\mu}{2 D}\right) v_{r}}-e^{\left(a-\frac{\mu}{2 D}\right) v_{t}}\right)+\frac{1}{a-\frac{\mu}{2 D}}\left(e^{\left(-a-\frac{\mu}{2 D}\right) v_{r}}-e^{\left(-a-\frac{\mu}{2 D}\right) v_{t}}\right)\right) \\
& K_{6}=\frac{1}{2}\left(\frac{1}{a+\frac{\mu}{2 D}}\left(e^{\left(a-\frac{\mu}{2 D}\right) v_{r}}-e^{\left(a-\frac{\mu}{2 D}\right) v_{t}}\right)-\frac{1}{a-\frac{\mu}{2 D}}\left(e^{\left(-a-\frac{\mu}{2 D}\right) v_{r}}-e^{\left(-a-\frac{\mu}{2 D}\right) v_{t}}\right)\right)
\end{aligned}
$$

\subsection{Discussion}

In this chapter we solve the linear response analytically in a spiking neuron model which includes explicitly the AP initiation process for an uncorrelated synaptic noise (white noise). The boundary induced artifacts are separated out mathematically. Our model includes the LIF model and the perfect integrator model 
as limiting cases. For a noise coded signal, the resulting response speed from the biophysically relevant part is characterized by the AP onset dynamics. The high frequency behavior dissociates from the membrane time constant and is determined solely by the shorter time constant that characterizes the AP initiation process. A fast AP onset can significantly enhance the cut-off frequencies. The stationary firing rate also plays a role in further lifting the cutoff frequency.

For a mean current coded signal, however, the AP onset rapidness is not so effective a factor in shifting the cutoff frequencies towards higher values. The membrane time constant plays a filtering effect no matter how large the AP onset rapidness is. Other factors and mechanisms have to be included to break the confinement by the membrane time constant. We recall that in the LIF model the response to a mean coded signal with a white noise is confined by the membrane time constant, but for a colored noise the confinement is broken and the response function does not decay at high signal frequencies (Brunel et al., 2001). Therefore the correlation in synaptic noise may play an important role in lifting the cutoff frequency, which will be considered in next chapter. 


\subsection{Appendix: parabolic cylinder functions}

The parabolic cylinder functions $U(a, x)$ and $V(a, x)$ are two independent solutions of the Weber's equation,

$$
\frac{d^{2} y}{d x^{2}}-\left(\frac{1}{4} x^{2}+a\right) y=0
$$

and are normalized to satisfy $U^{\prime}(a, x) V(a, x)-U(a, x) V^{\prime}(a, x)=-\sqrt{\frac{2}{\pi}}$, where the prime represents derivative with respect to $x$ (Abramowitz \& Stegun, 1970). Note that there is the relation $U(a, x)=D_{-a-\frac{1}{2}}(x)$ between the function $U(a, x)$ and the Whittake function $D_{a}(x)$. The function $V(a, x)$ is related to $U(a, x)$ by

$$
\pi V(a, x)=\Gamma\left(\frac{1}{2}+a\right)(\sin \pi a U(a, x)+U(a,-x))
$$

from which there are

$$
\begin{aligned}
& U(a,-x)=\frac{\pi}{\Gamma\left(\frac{1}{2}+a\right)} V(a, x)-\sin \pi a U(a, x) \\
& V(a,-x)=\frac{\Gamma\left(\frac{1}{2}+a\right)}{\pi} \cos ^{2} \pi a U(a, x)+\sin \pi a V(a, x) .
\end{aligned}
$$

The parabolic cylinder functions satisfies the following recurrence relations:

$$
\begin{aligned}
& U^{\prime}(a, x)+\frac{1}{2} x U(a, x)+\left(a+\frac{1}{2}\right) U(a+1, x)=0 \\
& U^{\prime}(a, x)-\frac{1}{2} x U(a, x)+U(a-1, x)=0 \\
& x U(a, x)-U(a-1, x)+\left(a+\frac{1}{2}\right) U(a+1, x)=0 \\
& V^{\prime}(a, x)-\frac{1}{2} x V(a, x)-\left(a-\frac{1}{2}\right) V(a-1, x)=0 \\
& V^{\prime}(a, x)+\frac{1}{2} x V(a, x)-V(a+1, x)=0 \\
& x V(a, x)-V(a+1, x)+\left(a-\frac{1}{2}\right) V(a-1, x)=0 .
\end{aligned}
$$

The parabolic cylinder functions $\psi_{1}(v)$ etc. used in solving the FPE for the 
linear response are defined by

$$
\begin{aligned}
\psi_{1}(v) & =U\left(-i \omega-\frac{1}{2},-\frac{v-\mu}{\sqrt{D}}\right)=D_{i \omega}\left(-\frac{v-\mu}{\sqrt{D}}\right) \\
\Phi_{1}(v) & =U\left(-i \omega+\frac{1}{2},-\frac{v-\mu}{\sqrt{D}}\right)=D_{i \omega-1}\left(-\frac{v-\mu}{\sqrt{D}}\right) \\
\Upsilon_{1}(v) & =U\left(-i \omega+\frac{3}{2},-\frac{v-\mu}{\sqrt{D}}\right)=D_{i \omega-2}\left(-\frac{v-\mu}{\sqrt{D}}\right) \\
\psi_{2}(v) & =\sqrt{\frac{\pi D}{2}} V\left(-i \omega-\frac{1}{2},-\frac{v-\mu}{\sqrt{D}}\right) \\
\psi_{3}(v) & =U\left(-i \omega / r+\frac{1}{2},-\frac{v-v_{t 0}+\mu / r}{\sqrt{D / r}}\right)=D_{i \omega / r-1}\left(-\frac{v-v_{t 0}+\mu / r}{\sqrt{D / r}}\right) \\
\Phi_{3}(v) & =U\left(-i \omega / r-\frac{1}{2},-\frac{v-v_{t 0}+\mu / r}{\sqrt{D / r}}\right)=D_{i \omega / r}\left(-\frac{v-v_{t 0}+\mu / r}{\sqrt{D / r}}\right) \\
\Upsilon_{3}(v) & =U\left(-i \omega / r-\frac{3}{2},-\frac{v-v_{t 0}+\mu / r}{\sqrt{D / r}}\right)=D_{i \omega / r+1}\left(-\frac{v-v_{t 0}+\mu / r}{\sqrt{D / r}}\right) \\
\psi_{4}(v) & =\sqrt{\frac{\pi D}{2 r}} V\left(-i \omega / r+\frac{1}{2},-\frac{v-v_{t 0}+\mu / r}{\sqrt{D / r}}\right) \\
\Phi_{4}(v) & =\sqrt{\frac{\pi D}{2 r}} V\left(-i \omega / r-\frac{1}{2},-\frac{v-v_{t 0}+\mu / r}{\sqrt{D / r}}\right) \\
\Upsilon_{4}(v) & =\sqrt{\frac{\pi D}{2 r}} V\left(-i \omega / r-\frac{3}{2},-\frac{v-v_{t 0}+\mu / r}{\sqrt{D / r}}\right) \\
Y_{1}(v) & =\psi_{3}(v) \psi_{4}\left(v_{b}\right)-\psi_{3}\left(v_{b}\right) \psi_{4}(v) \\
Y_{2}(v) & =\psi_{4}(v) \Phi_{3}\left(v_{b}\right)-i \omega / r \psi_{3}(v) \Phi_{4}\left(v_{b}\right) \\
Y_{3}(v) & =\psi_{4}(v) \Upsilon_{3}\left(v_{b}\right)-i \omega / r(1+i \omega / r) \psi_{3}(v) \Upsilon_{4}\left(v_{b}\right) .
\end{aligned}
$$

In deriving the large $v_{b}$ limit we introduce a new function

$$
\phi_{12}(v)=U\left(-i \frac{\omega}{r}+\frac{1}{2}, \frac{r\left(v-v_{t}\right)+\mu}{\sqrt{r D}}\right),
$$

which satisfies the same equation as $\psi_{3}(v)$ and $\psi_{4}(v)$.

Some useful relations can be derived from Eq. (2.89),

$$
\begin{aligned}
(1-i \omega) \Upsilon_{1}(v) & =\frac{v-\mu}{\sqrt{D}} \Phi_{1}(v)+\psi_{1}(v) \\
\Upsilon_{3}(v) & =-\frac{v-v_{t}+\mu / r}{\sqrt{D / r}} \Phi_{3}(v)-i \omega / r \psi_{3}(v) \\
(1+i \omega / r) \Upsilon_{4}(v) & =-\frac{v-v_{t}+\mu / r}{\sqrt{D / r}} \Phi_{4}(v)-\psi_{4}(v),
\end{aligned}
$$


and

$$
\begin{aligned}
& \psi_{4}(v) \Phi_{3}(v)-i \omega / r \psi_{3}(v) \Phi_{4}(v)=\sqrt{D / r} \\
& \psi_{4}^{\prime}(v) \Phi_{3}(v)-i \omega / r \psi_{3}^{\prime}(v) \Phi_{4}(v)=\frac{v-v_{t}+\mu / r}{2 \sqrt{D / r}} \\
& \psi_{4}(v) \Upsilon_{3}(v)-i \omega / r(1+i \omega / r) \psi_{3}(v) \Upsilon_{4}(v)=-\left(v-v_{t}+\mu / r\right) \\
& \psi_{4}^{\prime}(v) \Upsilon_{3}(v)-i \omega / r(1+i \omega / r) \psi_{3}^{\prime}(v) \Upsilon_{4}(v)=i \omega / r-\frac{\left(v-v_{t}+\mu / r\right)^{2}}{2 D / r} .
\end{aligned}
$$

For zero arguments, there are

$$
\begin{aligned}
U(a, 0) & =\frac{\sqrt{\pi}}{2^{\frac{1}{2} a+\frac{1}{4}} \Gamma\left(\frac{3}{4}+\frac{1}{2} a\right)} \\
U^{\prime}(a, 0) & =-\frac{\sqrt{\pi}}{2^{\frac{1}{2} a-\frac{1}{4}} \Gamma\left(\frac{1}{4}+\frac{1}{2} a\right)} \\
V(a, 0) & =\frac{2^{\frac{1}{2} a+\frac{1}{4}} \sin \pi\left(\frac{3}{4}-\frac{1}{2} a\right)}{\Gamma\left(\frac{3}{4}-\frac{1}{2} a\right)} \\
V^{\prime}(a, 0) & =\frac{2^{\frac{1}{2} a+\frac{3}{4}} \sin \pi\left(\frac{1}{4}-\frac{1}{2} a\right)}{\Gamma\left(\frac{1}{4}-\frac{1}{2} a\right)} .
\end{aligned}
$$

\section{Large argument expansion}

For $x \gg 0$,

$$
\begin{aligned}
U(a, x) & \sim e^{-x^{2} / 4} x^{-a-1 / 2}\left(1-\frac{\left(a+\frac{1}{2}\right)\left(a+\frac{3}{2}\right)}{2 x^{2}}+\ldots\right) \\
V(a, x) & \sim \sqrt{\frac{2}{\pi}} e^{x^{2} / 4} x^{a-1 / 2}\left(1+\frac{\left(a-\frac{1}{2}\right)\left(a-\frac{3}{2}\right)}{2 x^{2}}+\ldots\right) \\
U(a,-x) & \sim \frac{\sqrt{2 \pi}}{\Gamma\left(\frac{1}{2}+a\right)} e^{x^{2} / 4} x^{a-1 / 2}\left(1+\frac{\left(a-\frac{1}{2}\right)\left(a-\frac{3}{2}\right)}{2 x^{2}}+\ldots\right) \\
V(a,-x) & \sim \sqrt{\frac{2}{\pi}} \sin (\pi a) e^{x^{2} / 4} x^{a-1 / 2}\left(1+\frac{\left(a-\frac{1}{2}\right)\left(a-\frac{3}{2}\right)}{2 x^{2}}+\ldots\right),
\end{aligned}
$$

where the '...' represents higher order terms. The expansions for negative arguments are obtained by applying Eq. (2.88). 
Large $\omega$ expansion

For $|a| \gg 0$,

$D_{a}(x) \sim \frac{1}{\sqrt{2}} \exp \left(i x \sqrt{a}+\frac{a}{2}(\ln (a / e)-i \pi)\right) \times\left(\sum_{n=0}^{N-1} i^{n}(2 a)^{-n / 2} Q_{n}(x)+\mathcal{O}\left(|a|^{-N}\right)\right)$

where $Q_{0}(x)=1, Q_{1}(x)=-\frac{x\left(x^{2}-6\right)}{12 \sqrt{2}}$. 


\section{Chapter 3}

\section{Toward high cutoff frequencies for current coded signals}

In the preceding chapter, we found that the AP onset rapidness is an important factor for the dynamical response of a neuronal population. With the assumption of uncorrelated synaptic noise (Gaussian white noise), the cutoff frequency for a noise coded signal is found to be proportional to the AP onset rapidness, and therefore can be very large since the onset rapidness of cortical neurons was found to be very large in experiments (Naundorf et al., 2006). For a current coded signal, however, the response is confined by the membrane time constant. To achieve high cutoff frequencies for current coded signals as observed in experiments (Köndgen et al., 2008; Boucsein et al., 2009; Higgs \& Spain, 2009), other factors have to be taken into account.

One important factor we did not include until now is the correlation time in the synaptic noise. The synaptic inputs received by cortical neurons usually have a finite correlation time (colored noise) due to the synaptic decay time constant $\tau_{s}$. For real neurons, $\tau_{s}$ varies from several to hundreds of millisecond, induced by the fast (AMPA- and GABA $A_{-}$-mediated) and slow (NMDA- and GABA $B$ mediated) synapses. In the experiments which observed the high cutoff frequency phenomena, the injected currents are composed of a deterministic periodic part and a noise part with finite correlation time in a large range (Köndgen et al., 2008). On theoretical side, Brunel et al. (2001) found that the response function in the LIF model does not decay for a current coded signal with a colored noise, while for a white noise it decays as a power law. Therefore the inclusion of correlation in synaptic noise may have the effect of breaking the confinement by the membrane time constant. The flat response function reflects the infinite response speed at the threshold and therefore an artifact induced by the absorbing boundary, as for a noise coded signal with white noise in this model. For more realistic neuron models, like the EIF model and conductance based models, the transmission functions show a low-passed behavior and the cutoff frequencies could be enhanced with a large AP onset rapidness in the appearance of synaptic correla- 
tion (Fourcaud-Trocme et al., 2003: Naundorf et al., 2005: Fourcaud-Trocme \& Brunel, 2005).

The canonical Hodgkin-Huxley $(\mathrm{HH})$ type models seem not able to reproduce the unique features of the AP waveform observed in experiments, the very high onset rapidness together with large threshold variability, according to numerical simulations using multi-compartment models (Naundorf et al., 2006; Huang, 2009). In the experiments, the so called kink behavior was found to disappear by applying TTX to reduce the sodium channel density, and reappear by washing the drug out it (Naundorf et al., 2006). This suggests that there might be cooperativity among the sodium channels such that the opening of one channel per se can influence the open probability of its neighboring channels. In $\mathrm{HH}$ types neuron models, ion channels are taken as independent units with their open probability as a continuous function of the membrane potential (Hodgkin \& Huxley, 1952). Cooperativity among ion channels has not been observed directly for sodium channels, but has been observed for many other kinds of ion channels and neurons (Undrovinas et al.,. 1992: Molina et al., 2006; Marx et al.,. 1998; Bray \& Duke, 2004; Saito et al., 1988). The characterization of channel cooperativity has been investigated using one and two-dimensional Ising models (Liu \& Dilger, 1993). Under the assumption of channel cooperativity, a phase transition in conductance based models was found through a mean field approach when the interaction strength among sodium channels increases up to some critical value (Huang, 2009). The sodium channel activation curve is found not to increase continuously, but have a sharp threshold and changes from very low level to some finite value suddenly which results in a large AP onset rapidness. There are also some preliminary experimental results supporting the existence of a discontinuity in the sodium channel open probability (M. Gutnick, private communication).

In this chapter we will introduce a model that incorporates such a discontinuity in the spike generating current that would be induced by the cooperative opening of sodium channels. We will then investigate its functional effect on the dynamical response of a neuronal population. What we find here is a mutual enhancement between the step change and correlation in synaptic noise. If only with a step change, the response function for a current coded signal is still confined by the membrane time constant; but even a small subthreshold step change can have a significant effect for a colored noise although it might have quite weak effect for a white noise. The cutoff frequency can be very large with both a step change and a colored noise included. Therefore the appearance of a step change in the spike generating current effectively corresponds to a large AP onset rapidness. This has an intuitive explanation: If we push the step change to be large, the model will behave like the LIF model, which possesses a flat response function. So for a finite step we can expect a significant enhancement of the cutoff frequency with a colored noise. 


\subsection{Step change in sodium influx and its modeling}

To make analytical investigation possible, we will use a linear spike generating current same as that in the $r-\tau_{m}$ model in the preceding chapter. At a voltage $v_{0}$, which set the rheobase current, the intrinsic current has a step change $\Delta \dot{v}$. The dynamics is described by the following piecewise linear Langevin equation,

$$
\tau_{m} \dot{v}=f(v)+\mu+\sigma \eta(t)
$$

where

$$
f(v)= \begin{cases}-v, & -\infty<v \leq v_{0} \\ r\left(v-v_{t_{0}}\right), & v_{0}<v \leq v_{b}\end{cases}
$$

where $\tau_{m}$ is the membrane time constant and $r$ is the rapidness of AP onset. For convenience we take $\tau_{m}$ as the unit of time in analytical calculation. $\mu$ is the mean input current and $\sigma$ is the amplitude of synaptic noise. Here $\eta(t)$ is a Gaussian white noise which satisfies $\langle\eta(t)\rangle=0$ and $\left\langle\eta(t) \eta\left(t^{\prime}\right)\right\rangle=\tau_{m} \delta\left(t-t^{\prime}\right)$. The colored noise case will be studied later. At $v_{0}$ there is a step change, $r\left(v_{0}-v_{t 0}\right)-\left(-v_{0}\right)=$ $\Delta \dot{v}$, from which $v_{t 0}$ is given by

$$
v_{t 0}=\left(1+\frac{1}{r}\right) v_{0}-\frac{1}{r} \Delta \dot{v}
$$

We will use $v_{0}$ as the unit of voltage, $v_{0}=1$. When the membrane potential reaches the truncation point $v_{b}$ of the $\mathrm{AP}$ waveform, it is reset to a voltage $v_{r}$ and stays there for a refractory period $\tau_{r}$.

$\Delta \dot{v}$ can be quite large if there is some finite probability that a sodium channel open suddenly. Here is a simple estimation. Assuming there is a one percent chance that one channel per $\mu \mathrm{m}^{2}$ open suddenly, then the induced change in the rate of change of membrane potential, denoted as $\Delta \dot{v}$, will be $\Delta \dot{v} \approx 0.01 * g_{1} *$ $100 \mathrm{mV} / C_{m} \approx 1 \mathrm{mV} / \mathrm{ms}$, where $g_{1}=10 \mathrm{pS} / \mu \mathrm{m}^{2}$ is the conductance of a single sodium channel and $C_{m}=1 \mu \mathrm{F} / \mu \mathrm{m}^{2}$ is the specific capacitance of the membrane. Using, say, $10 \mathrm{mV}$ as the unit of voltage and $10 \mathrm{~ms}$ as the unite of time, this corresponds to $\Delta \dot{v} \simeq 1$ in the above model, which is large enough to have a significant effect on the cutoff frequency as we will see later.

The existence of a solution for the Langevin equation (3.1), however, is not guaranteed, since the dynamics at $v_{0}$ is not continuous. Here we will assume such a solution does exist. In the next chapter we will construct a more general model, which include the present model as a well-defined limit case and we will show that the two approaches give exactly the same results.

The Fokker-Planck equation (FPE) corresponding to Eq. (3.1) has the fol- 


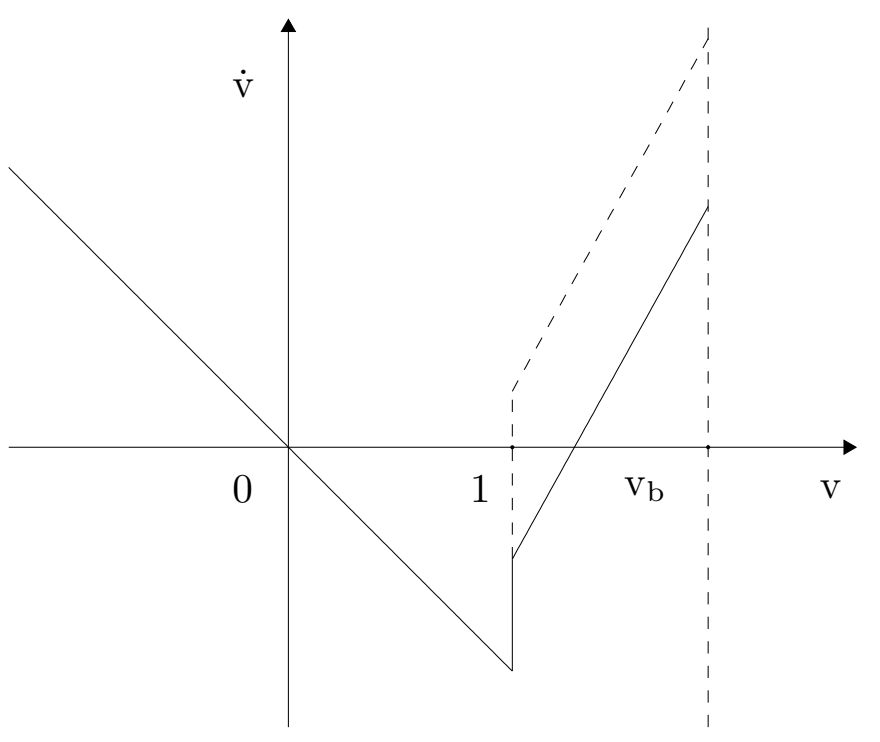

Figure 3.1: Illustration of the model.

lowing form

$$
\left\{\begin{array}{l}
\partial_{t} P_{1}(v, t)+\partial_{v}\left(-v+\mu-\frac{1}{2} \sigma^{2} \partial_{v}\right) P_{1}(v, t)=0, \quad-\infty<v \leq v_{0} \\
\partial_{t} P_{2}(v, t)+\partial_{v}\left(r\left(v-v_{t 0}\right)+\mu-\frac{1}{2} \sigma^{2} \partial_{v}\right) P_{2}(v, t)=0, v_{0}<v \leq v_{b}
\end{array}\right.
$$

where $P_{1}(v, t)$ and $P_{2}(v, t)$ are probability densities of the membrane potential $v$ with $-\infty<v \leq v_{0}$ and $v_{0}<v \leq v_{b}$ respectively. The probability currents are defined by

$$
\begin{aligned}
& J_{1}(v, t)=\left(-v+\mu-\frac{1}{2} \sigma^{2} \partial_{v}\right) P_{1}(v, t), \\
& J_{2}(v, t)=\left(r\left(v-v_{t 0}\right)+\mu-\frac{1}{2} \sigma^{2} \partial_{v}\right) P_{2}(v, t) .
\end{aligned}
$$

The probability density and the flux should be continuous at $v_{0}$, which requires

$$
\begin{aligned}
& P_{1}\left(v_{0}\right)=P_{2}\left(v_{0}\right) \\
& \partial_{v} P_{1}\left(v_{0}\right)+\frac{2 \Delta \dot{v}}{\sigma^{2}} P_{1}\left(v_{0}\right)=\partial_{v} P_{2}\left(v_{0}\right) .
\end{aligned}
$$

Other conditions are the same as that for the $r-\tau_{m}$ model in the previous chapter. The continuity and normalization conditions require that

$$
\begin{aligned}
P_{2}\left(v_{b}, t\right) & =0 \\
P_{1}\left(v_{r}^{+}, t\right)-P_{1}\left(v_{r}^{-}, t\right) & =0 \\
\partial_{v} P_{1}\left(v_{r}^{+}, t\right)-\partial_{v} P_{1}\left(v_{r}^{-}, t\right) & =\partial_{v} P_{2}\left(v_{b}, t-\tau_{r}\right), \\
\lim _{v \rightarrow-\infty} P_{1}(v, t) & =0 .
\end{aligned}
$$


These conditions set the boundary conditions of the system. The probability current into the absorbing boundary at $v_{b}$ gives the instantaneous firing rate

$$
\nu(t) \equiv J_{2}\left(v_{b}, t\right)=-\frac{1}{2} \sigma^{2} \partial_{v} P_{2}\left(v_{b}, t\right)
$$

\subsection{Stationary solutions}

When the mean input current $\mu$ is constant, the stationary probability densities, denoted as $P_{01}$ and $P_{02}$ for the two pieces respectively, satisfy

$$
\begin{array}{lc}
\left(-v+\mu-\frac{1}{2} \sigma^{2} \partial_{v}\right) P_{01}(v)=0, & -\infty<v \leq v_{r} \\
\left(-v+\mu-\frac{1}{2} \sigma^{2} \partial_{v}\right) P_{01}(v)=\nu_{0}, & v_{r}<v \leq v_{0} \\
\left(r\left(v-v_{t 0}\right)+\mu-\frac{1}{2} \sigma^{2} \partial_{v}\right) P_{02}(v)=\nu_{0}, & v_{0}<v \leq v_{b}
\end{array}
$$

where $\nu_{0}$ is the stationary firing rate.

Solving the above equation using the boundary conditions Eq. (3.6) and (3.7), the stationary probability densities are given by

$$
\begin{aligned}
P_{01}(v)= & \frac{2 \nu_{0}}{\sigma} e^{-\frac{1}{\sigma^{2}}(v-\mu)^{2}}\left(\int_{\frac{\max \left(v, v_{r}\right)-\mu}{\sigma}}^{\frac{v_{0}-\mu}{\sigma}} e^{y^{2}} d y\right. \\
& \left.+\frac{1}{2} \sqrt{\frac{\pi}{r}} e^{A}\left(\operatorname{Erfc}\left(\frac{\mu-v_{0}+\Delta \dot{v}}{\sqrt{r} \sigma}\right)-\operatorname{Erfc}\left(\frac{\mu+\dot{v}_{b}}{\sqrt{r} \sigma}\right)\right)\right) \\
P_{02}(v)= & \frac{\nu_{0}}{\sigma} \sqrt{\frac{\pi}{r}} e^{\frac{1}{r \sigma^{2}}\left(r\left(v-v_{t 0}\right)+\mu\right)^{2}}\left(\operatorname{Erfc}\left(\frac{\mu+r\left(v-v_{t 0}\right)}{\sqrt{r} \sigma}\right)-\operatorname{Erfc}\left(\frac{\mu+\dot{v}_{b}}{\sqrt{r} \sigma}\right)\right),
\end{aligned}
$$

where $\dot{v}_{b}=r\left(v_{b}-v_{t 0}\right)$ and

$$
A=\frac{1}{\sigma^{2}}\left(\left(v_{0}-\mu\right)^{2}+\frac{1}{r}\left(r\left(v_{0}-v_{t 0}\right)+\mu\right)^{2}\right)=\frac{1}{\sigma^{2}}\left(\left(v_{0}-\mu\right)^{2}+\frac{1}{r}\left(v_{0}-\Delta \dot{v}-\mu\right)^{2}\right) .
$$

From Fig. 3.2 we see that with the step change $\Delta \dot{v}$ increasing, the probability distribution of the membrane potential tends to localize near the resting potential.

By using the normalization condition of the probability density, $\nu_{0}$ reads

$$
\begin{aligned}
\nu_{0}^{-1}= & \sqrt{\pi} \int_{\left(\mu-v_{0}\right) / \sigma}^{\left(\mu-v_{r}\right) / \sigma} e^{y^{2}} \operatorname{Erfc}(y) d y \\
& +\frac{\pi}{2 \sqrt{r}} e^{A} \operatorname{Erfc}\left(\frac{\mu-v_{0}}{\sigma}\right)\left(\operatorname{Erfc}\left(\frac{\mu-v_{0}+\Delta \dot{v}}{\sqrt{r} \sigma}\right)-\operatorname{Erfc}\left(\frac{\mu+\dot{v}_{b}}{\sqrt{r} \sigma}\right)\right) \\
& +\frac{\sqrt{\pi}}{r} \int_{\left(\mu-v_{0}+\Delta \dot{v}\right) / \sqrt{r} \sigma}^{\left(\mu+\dot{v}_{b}\right) / \sqrt{r} \sigma} d x e^{x^{2}}\left(\operatorname{Erfc}(x)-\operatorname{Erfc}\left(\frac{\mu+\dot{v}_{b}}{\sqrt{r} \sigma}\right)\right)+\tau_{r} .
\end{aligned}
$$



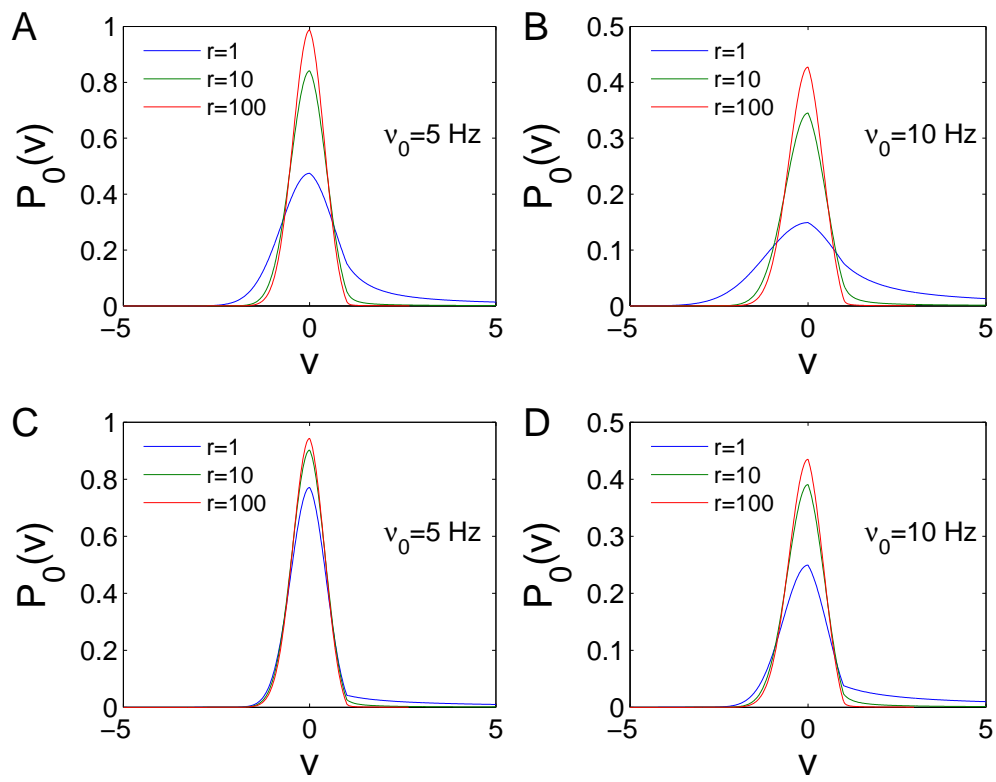

Figure 3.2: Dependence of the stationary probability density on the membrane potential for $\Delta \dot{v}=0.5(\mathrm{~A}, \mathrm{~B})$ and $\Delta \dot{v}=2(\mathrm{C}, \mathrm{D})$. Parameters used here and in other figures in this chapter: $\tau_{m}=10 \mathrm{~ms}, \mu=0, v_{r}=0, \tau_{r}=0$ and $v_{b}=10$ if not denoted otherwise.

When $r \rightarrow \infty$ or $\Delta \dot{v} \rightarrow \infty$, the stationary firing rate approaches to the result in the LIF model with a threshold at $v_{0}$, as given in Eq. (2.16).

We will define the dimensionless density $\tilde{P}_{01}(v)$, such that $P_{01}(v)=\frac{\nu_{0}}{\sqrt{D}} \tilde{P}_{01}(v)$ where $D=\frac{1}{2} \sigma^{2}$. In the large $v_{b}$ limit, $\tilde{P}_{01}(v)$ does not depend on $v_{b}$ and the values of the density and its derivative at $v_{0}$ will be needed later,

$$
\begin{aligned}
& P_{01}\left(v_{0}\right) \simeq \frac{\nu_{0}}{\sqrt{D}} \sqrt{\frac{\pi}{2 r}} e^{\frac{1}{r \sigma^{2}}\left(\mu-v_{0}+\Delta \dot{v}\right)} \operatorname{Erfc}\left(\frac{\mu-v_{0}+\Delta \dot{v}}{\sqrt{r} \sigma}\right)=\frac{\nu_{0}}{\sqrt{D}} \tilde{P}_{01}\left(v_{0}\right), \\
& P_{01}^{\prime}\left(v_{0}\right) \simeq-\frac{\nu_{0}}{D}\left(1+\frac{v_{0}-\mu}{\sqrt{D}} \tilde{P}_{01}\left(v_{0}\right)\right)=\frac{\nu_{0}}{\sqrt{D}} \tilde{P}_{01}^{\prime}\left(v_{0}\right) .
\end{aligned}
$$

When $\Delta \dot{v}$ is large,

$$
P_{01}(v) \simeq \nu_{0} e^{-\frac{1}{\sigma^{2}}(v-\mu)^{2}}\left(\frac{2}{\sigma} \int_{\frac{\max \left(v, v_{r}\right)-\mu}{\sigma}}^{\frac{v_{0}-\mu}{\sigma}} e^{y^{2}} d y+\frac{1}{\mu-v_{0}+\Delta \dot{v}} e^{\frac{1}{\sigma^{2}}\left(v_{0}-\mu\right)^{2}}\right),
$$

and

$$
\begin{aligned}
P_{01}\left(v_{0}\right) & \simeq \frac{\nu_{0}}{\mu-v_{0}+\Delta \dot{v}} \\
P_{01}^{\prime}\left(v_{0}\right) & \simeq-\frac{\nu_{0}}{D}\left(1+\frac{v_{0}-\mu}{\mu-v_{0}+\Delta \dot{v}}\right)
\end{aligned}
$$



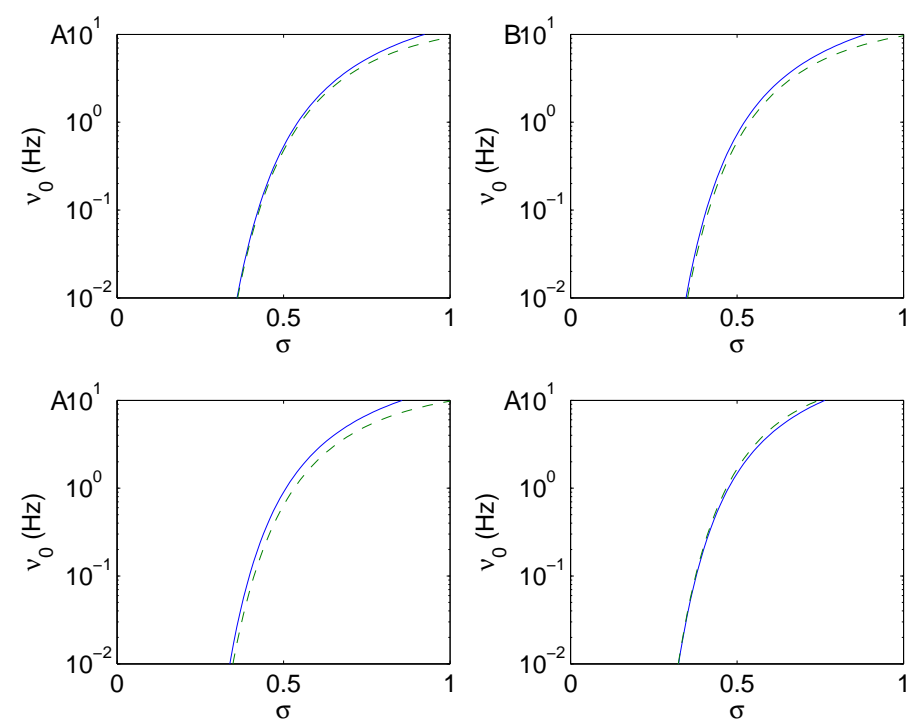

Figure 3.3: Dependence of the stationary firing rate on the noise strengh $\sigma$ for $\Delta \dot{v}=0(\mathrm{~A}), \Delta \dot{v}=0.5(\mathrm{~B}), \Delta \dot{v}=1(\mathrm{C})$ and $\Delta \dot{v}=5(\mathrm{D})$. Dashed lines are approximation from Eq. (3.16) and Eq. (3.17).

These resemble the probability density in the LIF model with a correction of the order of $1 / \Delta \dot{v}$. So the step change at $v_{0}$ behaves like a partial absorbing boundary. In deriving Eq. (3.13) we have used the asymptotic expansion for the complementary error function,

$$
\operatorname{Erfc}(x) \simeq \frac{1}{\sqrt{\pi} x} e^{-x^{2}}
$$

when $\mathrm{x}$ is large.

We will be most interested in the noise driven regime, $\mu-v_{0}<0$. The low rate approximation for the stationary firing rate now depends on the value of $\Delta \dot{v}$. When $\Delta \dot{v}+\mu<v_{0}$, we have

$$
\nu_{0}^{-1} \simeq \frac{\sqrt{\pi} \sigma}{v_{0}-\mu} e^{\frac{\left(v_{0}-\mu\right)^{2}}{\sigma^{2}}}+\frac{2 \pi}{\sqrt{r}} e^{\frac{1}{\sigma^{2}}\left(\left(v_{0}-\mu\right)^{2}+\frac{1}{r}\left(v_{0}-\Delta \dot{v}-\mu\right)^{2}\right)} .
$$

When $\Delta \dot{v}+\mu>v_{0}$, we have

$$
\begin{aligned}
\nu_{0}^{-1} & \simeq \sqrt{\pi} \sigma\left(\frac{1}{v_{0}-\mu}+\frac{1}{\mu-v_{0}+\Delta \dot{v}}\right) e^{\frac{\left(v_{0}-\mu\right)^{2}}{\sigma^{2}}} \\
& \simeq \frac{\sqrt{\pi} \sigma \Delta \dot{v}}{\left(v_{0}-\mu\right)\left(\mu-v_{0}+\Delta \dot{v}\right)} e^{\frac{\left(v_{0}-\mu\right)^{2}}{\sigma^{2}}} .
\end{aligned}
$$

Fig. 3.3 compares the approximation with the exact value of $\nu_{0}$. 


\subsection{Linear responses: directly solving the FPE}

The linear response can be solved analytically for the present model. Here we will solve the FPE at the linear order directly using the Green's function method. The procedure is similar to that in the last chapter. The results are expressed with the parabolic cylinder functions defined in the appendix in Chapter 2. Note that there needs a replacement for $v_{t} \rightarrow v_{t 0}$ in the definitions. Same as before, the arguments of the probability density and the parabolic cylinder functions are adopted at $v_{0}$ if not denoted explicitly.

\subsubsection{Linear response to a current coded signal}

When a weak sinusoidal signal is added to the mean input, $\mu(t)=\mu+\varepsilon \cos (\omega t)$, the instantaneous firing rate can then be written as $\nu(t)=\nu_{0}+\varepsilon\left|\nu_{1 c}(\omega)\right| \cos (\omega t-$ $\left.\phi_{c}(\omega)\right)$ when $\varepsilon$ is small. Here $\left|\nu_{1 c}(\omega)\right|$ is the transmission function and $\phi_{c}(\omega)$ is the phase lag, both of which are functions of signal frequency $\omega$.

At linear order, the probability densities can be expanded as

$$
\begin{aligned}
& P_{1}(v, t)=P_{01}(v)+\varepsilon P_{11}(v, t)+\cdots, \\
& P_{2}(v, t)=P_{02}(v)+\varepsilon P_{12}(v, t)+\cdots,
\end{aligned}
$$

where '...' represents higher order terms in $\varepsilon$. The FPE at the linear order in $\varepsilon$ are then given by

$$
\begin{aligned}
& \partial_{t} P_{11}(v, t)=\partial_{v}\left(v-\mu+D \partial_{v}\right) P_{11}(v, t)-\cos (\omega t) P_{01}^{\prime}(v), \quad-\infty<v \leq v_{0} \\
& \partial_{t} P_{12}(v, t)=\partial_{v}\left(-r v+r v_{t 0}-\mu+D \partial_{v}\right) P_{12}(v, t)-\cos (\omega t) P_{02}^{\prime}(v), v_{0}<v \leq v_{b}
\end{aligned}
$$

Factoring $P_{11}(v, t)$ and $P_{12}(v, t)$ as follows

$$
\begin{aligned}
P_{11}(v, t) & =\frac{1}{2}\left(e^{-i \omega t} q_{1}(v)+e^{i \omega t} q_{1}^{*}(v)\right) e^{-(v-\mu)^{2} / 4 D}, \\
P_{12}(v, t) & =\frac{1}{2}\left(e^{-i \omega t} q_{2}(v)+e^{i \omega t} q_{2}^{*}(v)\right) e^{\left(r v^{2}-2\left(r v_{t 0}-\mu\right) v\right) / 4 D},
\end{aligned}
$$

where the asterisks represent complex conjugate, we obtain

$$
\begin{aligned}
& D q_{1}^{\prime \prime}(v)-\left(\frac{(v-\mu)^{2}}{4 D}-i \omega-\frac{1}{2}\right) q_{1}(v)=e^{(v-\mu)^{2} / 4 D} P_{01}^{\prime}(v) \\
& \frac{D}{r} q_{2}^{\prime \prime}(v)-\left(\frac{\left(v-v_{t 0}+\mu / r\right)^{2}}{4 D / r}-\frac{i \omega}{r}+\frac{1}{2}\right) q_{2}(v)=\frac{1}{r} e^{-\left(r v^{2}-2\left(r v_{t 0}-\mu\right) v\right) / 4 D} P_{02}^{\prime}(v) .
\end{aligned}
$$


Substituting Eq. (3.18) and Eq. (3.20) into the boundary conditions Eq. (3.6. 3.7), we see that $q_{1}(v)$ and $q_{2}(v)$ satisfy the following boundary conditions,

$$
\begin{aligned}
\lim _{v \rightarrow-\infty} q_{1}(v) & =0, \\
q_{2}\left(v_{b}\right) & =0 \\
q_{1}\left(v_{0}\right) & =q_{2}\left(v_{0}\right) e^{\Delta_{02}}, \\
q_{1}^{\prime}\left(v_{0}\right)+\frac{\Delta \dot{v}}{2 D} q_{1}\left(v_{0}\right) & =q_{2}^{\prime}\left(v_{0}\right) e^{\Delta_{02}}, \\
q_{1}\left(v_{r}^{+}\right)-q_{1}\left(v_{r}^{-}\right) & =0 \\
q_{1}^{\prime}\left(v_{r}^{+}\right)-q_{1}^{\prime}\left(v_{r}^{-}\right) & =q_{2}^{\prime}\left(v_{b}\right) e^{\Delta_{01}+i \omega \tau_{r}},
\end{aligned}
$$

where

$$
\begin{aligned}
& \Delta_{01}=\left(\left(v_{r}-\mu\right)^{2}+\left(r v_{b}^{2}-2\left(r v_{t 0}-\mu\right) v_{b}\right)\right) / 4 D, \\
& \Delta_{02}=\left(\left(v_{0}-\mu\right)^{2}+\left(r v_{0}^{2}-2\left(r v_{t 0}-\mu\right) v_{0}\right)\right) / 4 D .
\end{aligned}
$$

Eq. (3.21) can be solved using the Green's function method. The Green's functions are the same as in Chapter 2. Following the same procedure, we then get

$$
q_{2}^{\prime}\left(v_{b}\right)=-\frac{1}{D} e^{-\Delta_{01}-i \omega \tau_{r}}(A+B)\left(\psi_{1}\left(v_{r}\right)+\left(Y_{1} \psi_{1}^{\prime}-Y_{1}^{\prime} \psi_{1}\right) e^{\Delta_{1}-\Delta_{0}-i \omega \tau_{r}}\right)^{-1},
$$

with

$$
\begin{aligned}
A= & \int_{-\infty}^{v_{0}} \psi_{1}(y) P_{01}^{\prime} e^{(y-\mu)^{2} / 2 \sigma^{2}} d y \\
B= & \frac{e^{\Delta_{02}}}{\psi_{3}\left(v_{b}\right)} \int_{v_{0}}^{v_{b}}\left(\left(\psi_{3} \psi_{1}^{\prime}-\psi_{3}^{\prime} \psi_{1}\right) Y_{1}(y)-\left(Y_{1} \psi_{1}^{\prime}-Y_{1}^{\prime} \psi_{1}\right) \psi_{3}(y)\right. \\
& \left.+\frac{\Delta \dot{v}}{2 D} \psi_{1}\left(\psi_{3} Y_{1}(y)-Y_{3} \psi_{3}(y)\right)\right) P_{02}^{\prime}(y) e^{-\left(r y^{2}-\left(r v_{t}-\mu\right) y\right) / 4 D} d y,
\end{aligned}
$$

where we have defined

$$
\begin{aligned}
& \Delta_{0}=\left(\left(v_{r}-\mu\right)^{2}-\left(v_{0}-\mu\right)^{2}\right) / 4 D \\
& \Delta_{1}=\left(\left(r v_{0}^{2}-2\left(r v_{t 0}-\mu\right) v_{0}\right)-\left(r v_{b}^{2}-2\left(r v_{t 0}-\mu\right) v_{b}\right)\right) / 4 D .
\end{aligned}
$$

At linear order in $\varepsilon$, the instantaneous firing rate is

$$
\begin{aligned}
\nu(t) & =-D \partial_{v} P\left(v_{b}, t\right) \\
& =\nu_{0}-\frac{\varepsilon}{2} D\left(e^{-i \omega t} q_{2}^{\prime}\left(v_{b}\right)+e^{i \omega t} q_{2}^{\prime *}\left(v_{b}\right)\right) e^{\left(r v_{b}^{2}-2\left(r v_{t}-\mu\right) v_{b}\right) / 4 D} \\
& =\nu_{0}+\varepsilon\left|\nu_{1 c}(\omega)\right| \cos \left(\omega t-\phi_{c}(\omega)\right),
\end{aligned}
$$


where $\nu_{1 c}(\omega)=-D q_{2}^{\prime}\left(v_{b}\right) e^{\left(r v_{b}^{2}-2\left(r v_{t 0}-\mu\right) v_{b}\right) / 4 D}$ is the complex response function.

The integrals in Eq. (3.30) can be performed explicitly using the properties of the parabolic cylinder functions, as in the last chapter. We then reach the following expressions for the response function $\nu_{1 c}(\omega)$,

$$
\begin{aligned}
\nu_{1 c}(\omega)= & \nu_{1 c}^{\text {Low }}(\omega)+\nu_{1 c}^{\text {High }}(\omega) \\
\nu_{1 c}^{\text {Low }}(\omega)= & \left(\psi_{1}\left(v_{r}\right) e^{\Delta_{0}+i \omega \tau_{r}}+\left(Y_{1} \psi_{1}^{\prime}-Y_{1}^{\prime} \psi_{1}+\frac{\Delta \dot{v}}{2 D} \psi_{1} Y_{1}\right) e^{\Delta_{1}}\right)^{-1} \\
& \times\left(\frac{i\left(\omega+\omega^{\prime}\right)}{(1-i \omega)\left(1+i \omega^{\prime}\right)}\left(\psi_{1} P_{01}-\sqrt{D} \Phi_{1} P_{01}^{\prime}\right)\right. \\
& \left.-\frac{i \omega}{1-i \omega} \frac{\nu_{0}}{\sqrt{D}} \Phi_{1}\left(v_{r}\right) e^{\Delta_{0}}-\frac{i \omega^{\prime}}{1+i \omega^{\prime}} \frac{\Delta \dot{v}}{\sqrt{D}} \Phi_{1} P_{01}\right), \\
\nu_{1 c}^{\text {High }}(\omega)= & \left(\psi_{1}\left(v_{r}\right) e^{\Delta_{0}+i \omega \tau_{r}}+\left(Y_{1} \psi_{1}^{\prime}-Y_{1}^{\prime} \psi_{1}+\frac{\Delta \dot{v}}{2 D} \psi_{1} Y_{1}\right) e^{\Delta_{1}}\right)^{-1} \\
& \times \frac{1}{1+i \omega^{\prime}} \frac{\nu_{0}}{\sqrt{r D}} e^{\Delta_{1}}\left(\psi_{1}^{\prime} Y_{2}-\psi_{1} Y_{2}^{\prime}+\frac{\Delta \dot{v}}{2 D} \psi_{1} Y_{2}\right),
\end{aligned}
$$

and $\phi_{c}(\omega)=\arg \left(\nu_{1 c}(\omega)\right)$ is the phase lag.

The decomposing into two additive terms is similar to that in the $r-\tau_{m}$ model. In Fig. 3.4 A, B and Fig. 3.5 A, B, the transmission function and the phase lag for a current coded signal with $r=1$ and $r=10$ respectively are shown. The numerical results are obtained by solving Eq. (3.1) directly using the RK2 method (Honeycutt, 1992a), which agree with the theoretical values very well.

\subsubsection{Linear response to a noise coded signal}

For a noise coded signal, there is $\sigma(t)=\sigma+\varepsilon \cos (\omega t)$. The probability densities can be divided into

$$
\begin{aligned}
& P_{1}(v, t)=P_{01}(v)+\varepsilon \sigma P_{21}(v, t)+\cdots, \\
& P_{2}(v, t)=P_{02}(v)+\varepsilon \sigma P_{22}(v, t)+\cdots,
\end{aligned}
$$

and the FPE becomes

$$
\begin{aligned}
& \partial_{t} P_{21}(v, t)=\partial_{v}\left(v-\mu+D \partial_{v}\right) P_{21}(v, t)+\cos (\omega t)\left(P_{01}^{\prime \prime}(v)+\delta\left(v-v_{0}\right) \frac{\Delta \dot{v}}{D} P_{01}\right), \\
& \partial_{t} P_{22}(v, t)=\partial_{v}\left(-r v+r v_{t}-\mu+D \partial_{v}\right) P_{22}(v, t)+\cos (\omega t) P_{02}^{\prime \prime}(v)
\end{aligned}
$$

where the Dirac $\delta$ function comes from the step change of the derivative of probability density at $v_{0}$, as given in Eq. (3.6).

Factoring the densities as

$$
\begin{aligned}
& P_{21}(v, t)=\frac{1}{2}\left(e^{-i \omega t} q_{1}(v)+e^{i \omega t} q_{1}^{*}(v)\right) e^{-(v-\mu)^{2} / 4 D}, \\
& P_{22}(v, t)=\frac{1}{2}\left(e^{-i \omega t} q_{2}(v)+e^{i \omega t} q_{2}^{*}(v)\right) e^{\left(r v^{2}-2\left(r v_{t}-\mu\right) v\right) / 4 D},
\end{aligned}
$$



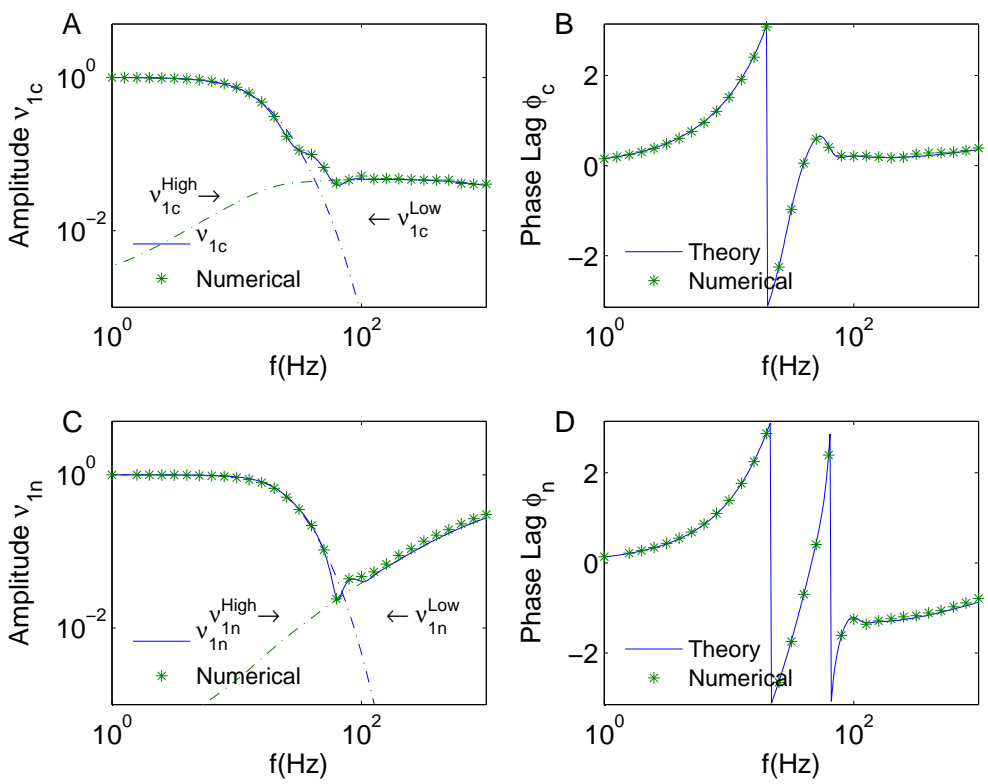

Figure 3.4: The normalized transmission functions $\nu_{1}(\omega) / \nu_{1}(0.1)$ and phase lags for a current coded signal (A,B) and for a noise coded signal (C,D) with $r=1$, $\Delta \dot{v}=0.5$ and $\nu_{0}=5 \mathrm{~Hz}$. The dashed and solid lines are theoretical results and the points are from numerical simulation using Eq. (2.53) and (2.54).
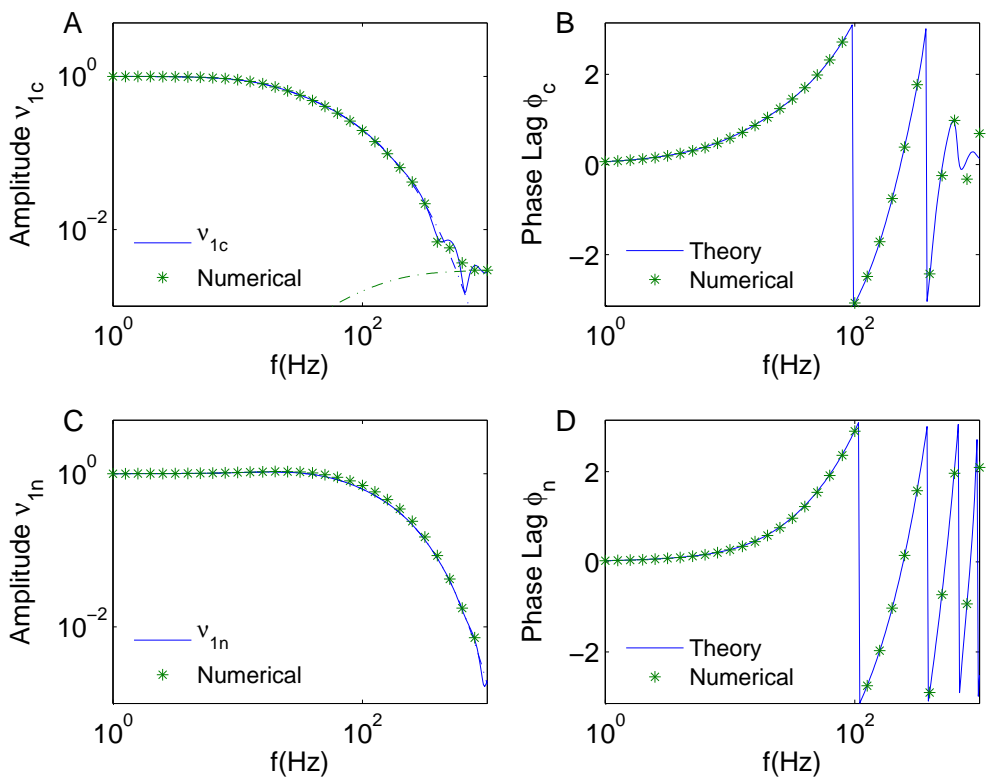

Figure 3.5: The normalized transmission functions $\nu_{1}(\omega) / \nu_{1}(0.1)$ and phase lags for a current coded signal $(\mathrm{A}, \mathrm{B})$ and for a noise coded signal $(\mathrm{C}, \mathrm{D})$ with $r=10$, $\Delta \dot{v}=0.5$ and $\nu_{0}=5 \mathrm{~Hz}$. 
we then get the following equations

$$
\begin{aligned}
& D q_{1}^{\prime \prime}(v)-\left(\frac{(v-\mu)^{2}}{4 D}-i \omega-\frac{1}{2}\right) q_{1}(v)=-e^{(v-\mu)^{2} / 4 D} \\
& \times\left(P_{01}^{\prime \prime}(v)+\delta\left(v-v_{0}\right) \frac{\Delta \dot{v}}{D} P_{01}(v)\right), \\
& \frac{D}{r} q_{2}^{\prime \prime}(v)-\left(\frac{\left(v-v_{t 0}+\mu / r\right)^{2}}{4 D / r}-\frac{i \omega}{r}+\frac{1}{2}\right) q_{2}(v)=\frac{-1}{r} e^{-\left(r v^{2}-2\left(r v_{t 0}-\mu\right) v\right) / 4 D} P_{02}^{\prime \prime}(v) .
\end{aligned}
$$

Solving Eq. (3.38) using the Green's function method, we get

$$
\begin{aligned}
q_{2}^{\prime}\left(v_{b}\right)= & \frac{1}{D} e^{-\Delta_{01}-i \omega \tau_{r}}\left(\psi_{1}\left(v_{r}\right)+\left(Y_{3} \psi_{1}^{\prime}-Y_{3}^{\prime} \psi_{1}\right) e^{\Delta_{02}-\Delta_{01}-i \omega \tau_{r}}\right)^{-1} \\
& \times\left(\frac{\nu_{0}}{D} \psi_{1}\left(v_{r}\right) e^{\left(v_{r}-\mu\right)^{2} / 4 D}+A+B+\frac{\Delta \dot{v}}{D} P_{01}\right)
\end{aligned}
$$

where the term $\frac{\Delta \dot{v}}{D} P_{01}\left(v_{0}\right)$ is from the $\delta$ function part for $P_{0}^{\prime \prime}(v)$ at $v_{0}$ and

$$
\begin{aligned}
A= & \int_{-\infty}^{v_{0}} \psi_{1}(y) P_{01}^{\prime \prime} e^{(y-\mu)^{2} / 2 \sigma^{2}} d y \\
B= & \frac{e^{\Delta_{02}}}{\psi_{3}\left(v_{b}\right)} \int_{v_{0}}^{v_{b}}\left(\left(\psi_{3} \psi_{1}^{\prime}-\psi_{3}^{\prime} \psi_{1}\right) Y_{3}(y)-\left(Y_{3} \psi_{1}^{\prime}-Y_{3}^{\prime} \psi_{1}\right) \psi_{3}(y)\right. \\
& \left.+\frac{\Delta \dot{v}}{2 D} \psi_{1}\left(\psi_{3} Y_{3}(y)-Y_{3} \psi_{3}(y)\right)\right) P_{02}^{\prime \prime}(y) e^{-\left(r y^{2}-\left(r v_{t 0}-\mu\right) y\right) / 4 D} d y
\end{aligned}
$$

The instantaneous firing rate is

$$
\begin{aligned}
\nu(t) & =-\left.(D+\varepsilon \sigma \cos (\omega t)) \partial_{v}\left(P_{02}+\varepsilon \sigma P_{22}\right)\right|_{v_{b}} \\
& =\nu_{0}+\varepsilon \sigma\left|\nu_{1 n}(\omega)\right| \cos \left(\omega t-\phi_{n}(\omega)\right),
\end{aligned}
$$

where $\nu_{1 n}(\omega)$ is the complex response function given by

$$
\nu_{1 n}(\omega)=\frac{\nu_{0}}{D}-D q_{2}^{\prime}\left(v_{b}\right) e^{\left(r v_{b}^{2}-2\left(r v_{t 0}-\mu\right) v_{b}\right) / 4 D} .
$$

Performing the integral in Eq. (3.40), the response function $\nu_{1 n}(\omega)$ is found 
to be decomposed into two terms,

$$
\begin{aligned}
\nu_{1 n}(\omega)= & \nu_{1 n}^{\text {Low }}(\omega)+\nu_{1 n}^{\text {High }}(\omega) \\
\nu_{1 n}^{\text {Low }}(\omega)= & \left(\psi_{1}\left(v_{r}\right) e^{\Delta_{0}+i \omega \tau_{r}}+\left(Y_{1} \psi_{1}^{\prime}-Y_{1}^{\prime} \psi_{1}+\frac{\Delta \dot{v}}{2 D} \psi_{1} Y_{1}\right) e^{\Delta_{1}}\right)^{-1} \\
& \times\left(\frac{i\left(\omega+\omega^{\prime}\right)}{(2-i \omega)\left(2+i \omega^{\prime}\right)}\left(-\frac{i \omega}{\sqrt{D}} \Phi_{1} P_{01}+2(i \omega-1) \Upsilon P_{01}^{\prime}\right)\right. \\
& \left.+\frac{i \omega(i \omega-1)}{2-i \omega} \frac{\nu_{0}}{D} \Upsilon\left(v_{r}\right) e^{\Delta_{0}}+\frac{\Delta \dot{v}}{D} \frac{i \omega^{\prime}}{2+i \omega^{\prime}}(i \omega-1) \Upsilon_{1} P_{01}\right), \\
\nu_{1 n}^{\text {High }}(\omega)= & \left(\psi_{1}\left(v_{r}\right) e^{\Delta_{0}+i \omega \tau_{r}}+\left(Y_{1} \psi_{1}^{\prime}-Y_{1}^{\prime} \psi_{1}+\frac{\Delta \dot{v}}{2 D} \psi_{1} Y_{1}\right) e^{\Delta_{1}}\right)^{-1} \\
& \times \frac{1}{2+i \omega^{\prime}} \frac{\nu_{0}}{D} e^{\Delta_{1}}\left(Y_{3} \psi_{1}^{\prime}-Y_{3}^{\prime} \psi_{1}+\frac{\Delta \dot{v}}{2 D} \psi_{1} Y_{3}\right),
\end{aligned}
$$

and $\phi_{n}(\omega)=\arg \left(\nu_{1 n}(\omega)\right)$ is the phase lag. Fig. 3.4 C, D and Fig. 3.5 C, D, show the transmission function and the phase lag for a noise coded signal with $r=1$ and $r=10$ respectively. The numerical results agree with the theoretical values very well.

\subsection{Large $v_{b}$ limit and separation of boundary in- duced artifacts}

When the absorbing boundary is far from the AP initiation point, i.e. $v_{b} \gg v_{0}$, we can apply the large argument expansion to parabolic cylinder functions with argument at $v_{b}$. The expression for the linear response is simplified and the dependence on $v_{b}$ becomes transparent. Without any change from Section 2.4, we have

$$
\begin{aligned}
& Y_{1}\left(v_{0}\right) e^{\Delta_{1}} \simeq-\sqrt{D / r} \phi_{12}\left(v_{0}\right) e^{x_{1}^{2} / 4-i \omega / r \ln x} \\
& Y_{2}\left(v_{0}\right) e^{\Delta_{1}} \simeq-\sqrt{D / r} i \omega / r \phi_{12}\left(v_{0}\right) e^{x_{1}^{2} / 4-i \omega / r \ln x} \cdot \frac{1}{x} \\
& Y_{3}\left(v_{0}\right) e^{\Delta_{1}} \simeq \sqrt{D / r} i \omega(1+i \omega / r) \phi_{12}\left(v_{0}\right) e^{x_{1}^{2} / 4-i \omega / r \ln x} \cdot \frac{1}{x^{2}},
\end{aligned}
$$

where $x=\frac{r\left(v_{b}-v_{t 0}\right)+\mu}{\sqrt{r D}}, x_{1}=\frac{-v_{0}+\Delta \dot{v}+\mu}{\sqrt{r D}}$ and $\phi_{12}(v)=U\left(-i \omega / r+\frac{1}{2}, \frac{r\left(v-v_{t 0}\right)+\mu}{\sqrt{r D}}\right)$. In the large $v_{b}$ limit, $P_{0}\left(v_{0}\right)$ and $P_{0}^{\prime}\left(v_{0}\right)$ are given in Eq. (3.12). $\nu_{1 c}(\omega)$ is then 
simplified to

$$
\begin{aligned}
\nu_{1 c}^{\text {Low }}(\omega) \simeq & \frac{\nu_{0}}{\sqrt{D}}\left(\psi_{1}\left(v_{r}\right) e^{\Delta_{0}+i \omega \tau_{r}}-\sqrt{\frac{D}{r}}\left(\phi_{12} \psi_{1}^{\prime}-\phi_{12}^{\prime} \psi_{1}+\frac{\Delta \dot{v}}{2 D} \phi_{12} \psi_{1}\right) e^{x_{1}^{2} / 4-i \omega / r \ln x}\right)^{-1} \\
& \times\left(\frac{i \omega(1+1 / r)}{(1-i \omega)(1+i \omega / r)}\left(\psi_{1} \tilde{P}_{01}-\sqrt{D} \Phi_{1} \tilde{P}_{01}^{\prime}\right)-\frac{i \omega}{1-i \omega} \Phi_{1}\left(v_{r}\right) e^{\Delta_{0}}\right. \\
& \left.\quad-\frac{i \omega}{r+i \omega} \frac{\Delta \dot{v}}{\sqrt{D}} \Phi_{1} \tilde{P}_{01}\right) \\
\nu_{1 c}^{H i g h}(\omega) \simeq & \frac{\nu_{0}}{r\left(v_{b}-v_{t}\right)+\mu} \frac{-i \omega / r}{1+i \omega / r} \times \\
& \frac{\sqrt{D / r}\left(\phi_{12} \psi_{1}^{\prime}-\phi_{12}^{\prime} \psi_{1}+\frac{\Delta \dot{v}}{2 D} \phi_{12} \psi_{1}\right) e^{x_{1}^{2} / 4-i \omega / r \ln x}}{\psi_{1}\left(v_{r}\right) e^{\Delta_{0}+i \omega \tau_{r}}-\sqrt{D / r}\left(\phi_{12} \psi_{1}^{\prime}-\phi_{12}^{\prime} \psi_{1}+\frac{\Delta \dot{v}}{2 D} \phi_{12} \psi_{1}\right) e^{x_{1}^{2} / 4-i \omega / r \ln x}} \cdot(3.46)
\end{aligned}
$$

For $\nu_{1 n}(\omega)$, there is

$$
\begin{aligned}
\nu_{1 n}^{\text {Low }}(\omega) \simeq & \frac{\nu_{0}}{D}\left(\psi_{1}\left(v_{r}\right) e^{\Delta_{0}+i \omega \tau_{r}}-\sqrt{\frac{D}{r}}\left(\phi_{12} \psi_{1}^{\prime}-\phi_{12}^{\prime} \psi_{1}+\frac{\Delta \dot{v}}{2 D} \phi_{12} \psi_{1}\right) e^{x_{1}^{2} / 4-i \omega / r \ln x}\right)^{-1} \\
& \times\left(\frac{i \omega(1+1 / r)}{(2-i \omega)(2+i \omega / r)}\left(-i \omega \Phi_{1} \tilde{P}_{01}+2(i \omega-1) \sqrt{D} \Upsilon \tilde{P}_{01}^{\prime}\right)\right. \\
& \left.+\frac{i \omega(i \omega-1)}{2-i \omega} \Upsilon\left(v_{r}\right) e^{\Delta_{0}}+\frac{i \omega^{\prime}(i \omega-1)}{2+i \omega^{\prime}} \frac{\Delta \dot{v}}{\sqrt{D}} \Upsilon_{1} \tilde{P}_{01}\right) \\
\nu_{1 n}^{H i g h}(\omega) \simeq & \frac{\nu_{0}}{\left(r\left(v_{b}-v_{t 0}\right)+\mu\right)^{2}} \frac{i \omega(1+i \omega / r)}{2+i \omega / r} \times \\
& \frac{\sqrt{D / r}\left(\phi_{12} \psi_{1}^{\prime}-\phi_{12}^{\prime} \psi_{1}+\frac{\Delta \dot{v}}{2 D} \phi_{12} \psi_{1}\right) e^{x_{1}^{2} / 4-i \omega / r \ln x}}{\psi_{1}\left(v_{r}\right) e^{\Delta_{0}+i \omega \tau_{r}}-\sqrt{D / r}\left(\phi_{12} \psi_{1}^{\prime}-\phi_{12}^{\prime} \psi_{1}+\frac{\Delta \dot{v}}{2 D} \phi_{12} \psi_{1}\right) e^{x_{1}^{2} / 4-i \omega / r \ln x}} .
\end{aligned}
$$

When $\omega$ is not small $(\omega \geq 1)$, the contribution from $\psi_{1}\left(v_{r}\right)$ term is negligible compared to the other term in the denominator in the expressions of the linear response. We then have

$$
\begin{aligned}
\nu_{1 c}^{H i g h}(\omega) & \simeq \frac{\nu_{0}}{r\left(v_{b}-v_{t 0}\right)+\mu} \frac{i \omega}{r+i \omega} \\
\nu_{1 n}^{H i g h}(\omega) & \simeq-\frac{\nu_{0}}{\left(r\left(v_{b}-v_{t 0}\right)+\mu\right)^{2}} \frac{i \omega(1+i \omega / r)}{2+i \omega / r}
\end{aligned}
$$

We see that $\nu_{1}^{\text {High }}(\omega)$ are strongly suppressed when $v_{b}$ is large. The $\nu_{1}^{\text {Low }}(\omega)$ parts, on the contrary, depend on $v_{b}$ mainly through a frequency dependent phase lag, characterizing the time lag to the truncation point $v_{b}$ of the AP.

The decomposing of $\nu_{1}(\omega)$ into two additive components has exactly the features required for the separation of boundary induced artifacts, as outlined in section 2.4.2 in Chapter 2. Therefore, we have $\nu_{1}^{\text {High }}(\omega)=\nu_{1}^{a b s}(\omega)$ and $\nu_{1}^{\text {Low }}(\omega)=\nu_{1}^{\text {phy }}(\omega)$. The biophysically meaningful predictions of the model thus comes from $\nu_{1}^{\text {Low }}(\omega)$. 


\subsection{Linear response: limit behavior}

\subsubsection{Large $r$ limit and large $\omega$ limit}

In the large $r$ limit, the $\Delta \dot{v}$ term in the linear response approaches to zero as $\frac{1}{\sqrt{r}}$. Without any change, the argument for the $r-\tau_{m}$ model can be used here. The $\nu_{1}^{\text {High }}(\omega)$ parts decay to zero and the $\nu_{1}^{\text {Low }}(\omega)$ parts of the linear response give exactly the results for the LIF model when $r \rightarrow \infty$.

By using the large $\omega$ expansion of the parabolic cylinder functions, the high frequency limit of the linear response can be obtained: $\nu_{1 c}(\omega) \rightarrow \frac{\nu_{0}}{\sqrt{D}} \frac{1}{\sqrt{\omega}} e^{i \pi / 4}$ and $\nu_{1 n}(\omega) \rightarrow \frac{\nu_{0}}{D}$, the same as the LIF model. These are provided solely by $\nu_{1}^{H i g h}(\omega)$, while the $\nu_{1}^{\text {Low }}(\omega)$ parts decay to zero exponentially. In the high signal frequency limit, the linear response is shaped exclusively by the absorbing boundary at $v_{b}$ and therefore is expected to be similar for every integrate-and-fire model with finite $v_{b}$.

\subsubsection{Large $\Delta \dot{v}$ limit}

In the large $\Delta \dot{v}$ limit, we can start from the large $v_{b}$ limit Eq. (3.45) and (3.47), since the large $v_{b}$ limit is obtained through the large argument expansion about $\frac{r\left(v_{b}-v_{t}\right)}{\sqrt{r D}}$ which is large when $\Delta \dot{v}$ is large. The large arguments expansion now is further applied to the parabolic cylinder functions with argument at $\frac{r\left(v_{0}-v_{t 0}\right)}{\sqrt{r D}}$. It is easy to check $\phi_{12}\left(v_{0}\right) e^{x_{1}^{2} / 4-i \omega / r \ln x} \rightarrow 0$ as $1 / \Delta \dot{v}$ and

$$
\left(-\phi_{12}^{\prime}\left(v_{0}\right)+\frac{\Delta \dot{v}}{2 D} \phi_{12}\left(v_{0}\right)\right) e^{x_{1}^{2} / 4-i \omega / r \ln x} \rightarrow \sqrt{\frac{r}{D}} .
$$

If we start from the accurate formulas for linear responses, Eq. (3.33) and (3.43), we will find that $Y_{1}\left(v_{0}\right) e^{\Delta_{1}} \rightarrow 0$ as $1 / \Delta \dot{v}$ and $\left(-Y_{1}^{\prime}\left(v_{0}\right)+\frac{\Delta \dot{v}}{2 D} Y_{1}\left(v_{0}\right)\right) e^{\Delta_{1}} \rightarrow 1$.

Additionally, note that when $\Delta \dot{v} \rightarrow \infty$,

$$
\begin{aligned}
& P_{01}^{\prime}\left(v_{0}\right) \rightarrow-\frac{\nu_{0}}{D}, \\
& \Delta \dot{v} P_{01}\left(v_{0}\right) \rightarrow-\nu_{0} .
\end{aligned}
$$

We then find that the $\nu_{1}^{H i g h}(\omega)$ parts approach to zero as $1 / \Delta \dot{v}$ and $1 / \Delta \dot{v}^{2}$ for a current coded signal and a noise coded signal respectively. Finally we have

$$
\begin{aligned}
\nu_{1 c} & \rightarrow \frac{\nu_{0}}{\sqrt{D}} \frac{i \omega}{i \omega-1}\left(\Phi_{1}-\Phi_{1}\left(v_{r}\right) e^{\Delta_{0}}\right) /\left(\psi_{1}-\psi_{1}\left(v_{r}\right) e^{\Delta_{0}}\right), \\
\nu_{1 n} & \rightarrow \frac{\nu_{0}}{D} \frac{i \omega(i \omega-1)}{2-i \omega}\left(\Upsilon_{1}-\Upsilon_{1}\left(v_{r}\right) e^{\Delta_{0}}\right) /\left(\psi_{1}-\psi_{1}\left(v_{r}\right) e^{\Delta_{0}}\right),
\end{aligned}
$$

which are solely from the $\nu_{1}^{\text {Low }}$ parts and are exactly the linear response for the LIF model with a threshold at $v_{0}$. Therefore, if we let $\Delta \dot{v} \rightarrow \infty$, the linear 

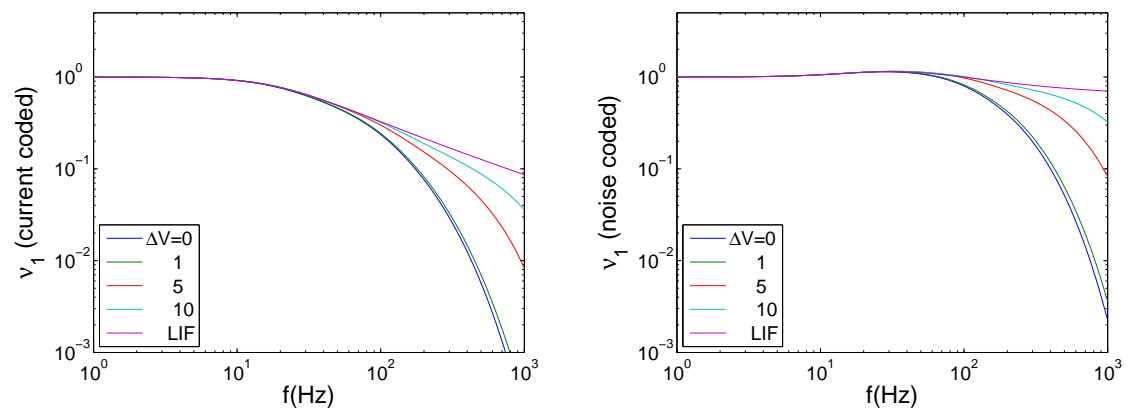

Figure 3.6: The normalized transmission functions $\nu_{1}(\omega) / \nu_{1}(0.1)$ with step change $\Delta \dot{v}=1,5,10$ and for the corresponding LIF model results.

responses to both a current coded signal and a noise coded signal will approach the results of the LIF model. For a finite $\Delta \dot{v}$, the step change is expected to play the role of an absorbing boundary partially and will shape the linear response significantly.

\subsubsection{High frequency behavior: effect of the step change with white noise}

Fig. 3.6 shows the dependence of the transmission function on $\Delta \dot{v}$, and how the linear response change with the inclusion of a step change $\Delta \dot{v}$ at $v_{0}$. We have proven that when $\Delta \dot{v} \rightarrow \infty$, the linear responses approach that of the LIF model with a threshold at $v_{0}$. Fig. 3.6 shows this behavior clearly. The cutoff frequency for a noise coded signal can be shifted to arbitrary high signal frequency with a large $\Delta \dot{v}$. For a current coded signal, the effect of increasing $\Delta \dot{v}$ is not significant and the membrane time constant still confines the high frequency response.

\subsection{Effect of correlation time $\tau_{s}$}

\subsubsection{Modeling correlated synaptic noise}

The postsynaptic input usually have a finite correlation time, due to the filtering of the afferent spike trains by the synapses. This can be approximated well by a deterministic mean input and a color noise with a correlation time $\tau_{s}$ (Tuckwell, 1988).

For a colored noise, the dynamics is described by the following two dimensional Langevin equation,

$$
\begin{aligned}
\tau_{m} \dot{v} & =f(v)+\mu+\epsilon(t), \\
\tau_{s} \dot{\epsilon} & =-\epsilon+\sigma \eta(t),
\end{aligned}
$$


where

$$
f(v)= \begin{cases}-v, & -\infty<v \leq v_{0} \\ r\left(v-v_{t_{0}}\right), & v_{0}<v \leq v_{b}\end{cases}
$$

Here $\eta(t)$ is a Gaussian white noise which satisfies $\langle\eta(t)\rangle=0$ and $\left\langle\eta(t) \eta\left(t^{\prime}\right)\right\rangle=$ $\tau_{m} \delta\left(t-t^{\prime}\right) . \epsilon$ is a colored noise described by an Ornstein-Uhlenbeck (OU) process with $\langle\epsilon(t)\rangle=0$ and $\left\langle\epsilon(t) \epsilon\left(t^{\prime}\right)\right\rangle=\frac{\sigma^{2} \tau_{m}}{2 \tau_{s}} \exp \left(-\left|t-t^{\prime}\right| / \tau_{s}\right)$.

For the dynamical response, a small sinusoidal signal $\varepsilon \cos (\omega t)$ will be added, such that $\mu(t)=\mu+\varepsilon \cos (\omega t)$ for a current coded signal and and $\epsilon(t) \rightarrow(1+$ $\varepsilon \cos (\omega t)) \epsilon(t)$. For a current coded signal, the Langevin equation Eq. (3.53) will be solved using the second order Runge-Kutta method (Honeycutt, 1992b) and the linear response are obtained through Eq. (2.53). While for a noise coded signal, the algorism in Honeycutt (1992b) is not applicable, since now the variance of the colored noise is time dependent. We will use the Euler method (see e.g., Milshtein \& Tret'yakov (1994)) for a noise coded signal.

\subsubsection{Stationary firing rate $\nu_{0}$}

In Fig. 3.7, we show the dependence of the stationary firing rate $\nu_{0}$ on the correlation time $\tau_{s}$ for different step change $\Delta \dot{v}$. The AP onset rapidness $r$ is fixed to be $r=10$. We see that the correlation time has a significant impact on $\nu_{0}$, but the influence of the step change on $\nu_{0}$ is quite weak. When $\Delta \dot{v}$ changes from 0 to 5 , there is only a weak influence when $\tau_{s}$ is small. We will see later that even a small step change, has a significant influence on the linear response.

\subsubsection{Interplay of $\tau_{s}$ and $r$ without a step change}

In the LIF model, the linear response for a mean coded signal with colored noise has a flat (not attenuated at high signal frequency) transfer function. The present model approaches to the LIF model when $r \rightarrow \infty$ or $\Delta \dot{v} \rightarrow \infty$. So the cutoff frequencies are expected to increase with both $r$ and $\Delta \dot{v}$. Let us first check how the response function changes with the increasing of $r$ when $\Delta \dot{v}=0$. For a fixed $\tau_{s}$, the cutoff frequencies are shifted to higher values with the increasing of $r$, as seen from Fig. 3.8. As in the white noise case, the absorbing boundary introduces artifacts for the high frequency response. But as shown in Fig. 3.8, the artificial part of the linear response is significant only for small $r$ and is suppressed by a large $v_{b}$ as we found in the white noise case (compare with Fig. 2.10). When $r$ is not small $(r>1)$, the transmission functions for the physiological relevant signal frequencies are not influenced by the increase of $v_{b}$. When $r \rightarrow \infty$, the model reduces to the LIF model and possesses a flat response function, with the limit value calculated analytically in Brunel et al. (2001) for small $\tau_{s}, \lim _{\omega \rightarrow \infty} \nu_{1 c}(\omega)=\frac{A \nu_{0}}{\sigma} \sqrt{\frac{\tau_{s}}{\tau_{m}}}$, where $A \approx 1.3238$. 

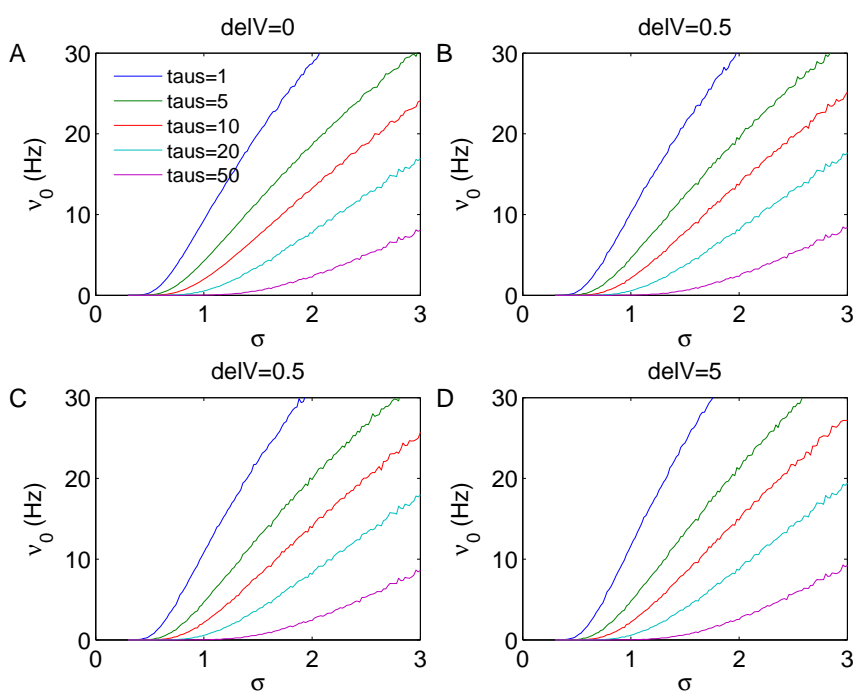

Figure 3.7: The dependence of stationary firing rate $\nu_{0}$ on the correlation time $\tau_{s}$ with different step change $\Delta \dot{v}$. From A to D the step change are : A, $\Delta \dot{v}=0$, $\mathrm{B}, \Delta \dot{v}=0.5, \mathrm{C}, \Delta \dot{v}=1, \mathrm{D}, \Delta \dot{v}=5$. In each figures the curves from upper to lower are for $\tau_{s}=1,5,10,20 \mathrm{~ms}$ respectively. $r$ is fixed to be 10 .

Fig. 3.9 shows the transmission functions with $r$ fixed. With the increasing of $\tau_{s}$, the transmission function is shifted towards to high frequencies. The enhancement is significant only for a large AP onset rapidness. The high cutoff frequencies can be readily understood by increasing $r$ or including a step change $\Delta \dot{v}$ in the white noise case for a noise coded signal. A finite correlation time can further enhance the high frequency response to a noise coded signal, but the effect is not significant, as can be seen from Fig. 3.10. We will focus on the mean current coded signal here

\subsubsection{Interplay of $\tau_{s}$ and $\Delta \dot{v}$}

We have proven that when $\Delta \dot{v} \rightarrow \infty$, the stationary and the linear responses of the model reduce to that for the LIF model in the white noise case. So with the including of correlation in synaptic noise, the cutoff frequencies are expected to be further enhanced by $\Delta \dot{v}$ and resemble the LIF model which has a flat transfer function for the colored noise.

Fig. 3.11 shows that when $\tau_{s}$ and $r$ are fixed, the cutoff frequencies increase with $\Delta \dot{v}$. When $r$ is large, the attenuation of the transmission function in the biophysically relevant frequency range has already become quite weak, so the step changes do not have much impact there. Roughly speaking, a step change in the spike generating current effectively increases the AP onset rapidness and provides an efficient way to enhance the cutoff frequency in the dynamical response under 

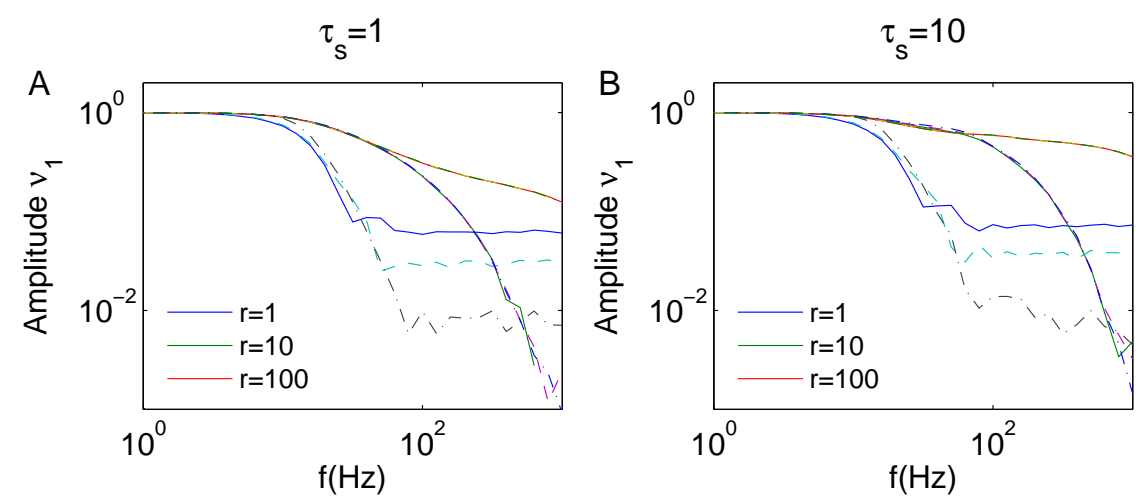

Figure 3.8: The dependence of the the transmission function $\left|\nu_{1 c}(\omega) / \nu_{1 c}(0.1)\right|$ on the boundary $v_{b}$ for $\tau_{s}=1,10 \mathrm{~ms}$ and $r=1,10,100$. The solid, dashed and dash-dot lines are for $v_{b}=10,20,100$ respectively. Parameter used: $\mu=0$, $\nu_{0}=5 \mathrm{~Hz}$.
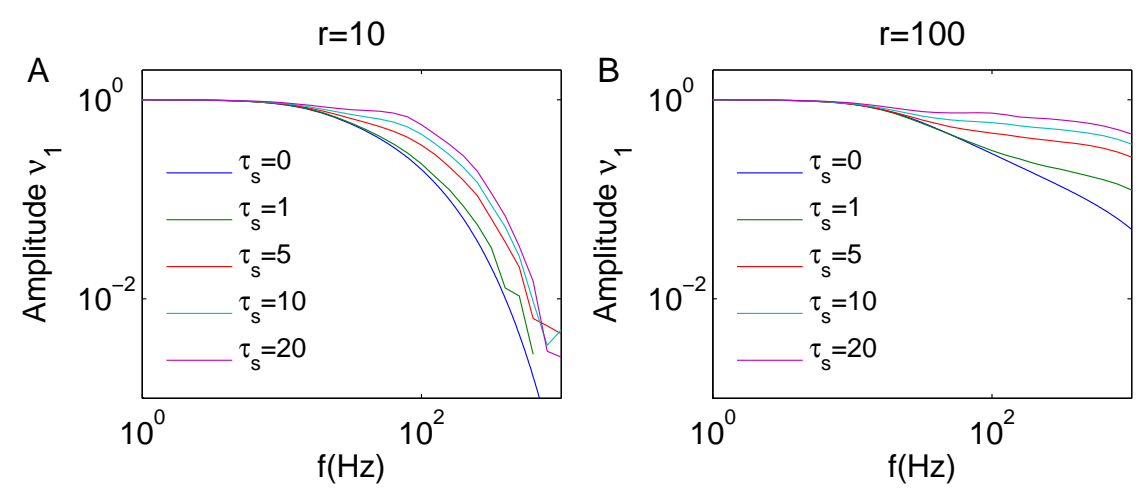

Figure 3.9: Variation of the normalized transmission function $\left|\nu_{1 c}(\omega) / \nu_{1 c}(0.1)\right|$ with increasing $\tau_{s}$ for $r=10,100$ when $\Delta \dot{v}=0$. Parameter used here and in the following: $\mu=0, v_{b}=10, \nu_{0}=5 \mathrm{~Hz}$. 

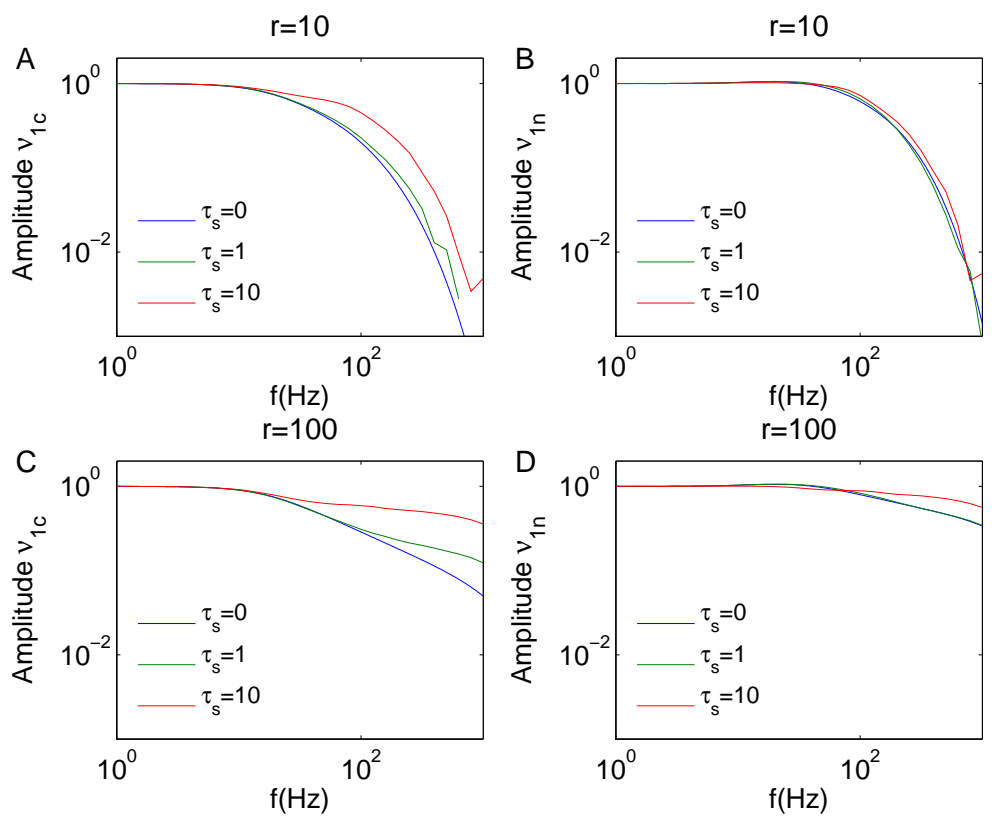

Figure 3.10: Dependence of the normalized transmission function $\left|\nu_{1}(\omega) / \nu_{1}(0.1)\right|$ on $\tau_{s}$ for a current coded signal $(\mathrm{A}, \mathrm{C})$ and a noise coded signal $(\mathrm{B}, \mathrm{D})$. A, B, $r=10 ; \mathrm{C}, \mathrm{D}, r=100$.

the condition of correlated noise. For a given step change and AP onset rapidness, Fig. 3.12 shows the responses in high frequency range are significant enhanced and the cutoff frequency is shifted to hundreds Hz. Comparing Fig. 3.9 with Fig. 3.12, we can see clearly a similar effect in increasing the high frequency response by a large onset rapidness and a step change in the spike generating current.

\subsection{Discussion}

In this chapter we have studied the influence of a step change in the AP generating current and of the correlation in the synaptic current on the dynamical response. If the correlation time of the synaptic current is ignored, the linear response will approach the LIF model when the step change is large. Therefore the cutoff frequency is still confined by the membrane time constant in the white noise case. Note that for the linear response we mean only the biophysically meaningful part with the artifactual parts from the absorbing boundary eliminated. When the correlation time in synaptic noise is taken into account, however, both a large AP onset rapidness and a step change in the spike generating current have the effect of breaking the confinement by the membrane time constant. The response shows a low pass behavior, but with the cutoff frequencies shifted towards the high frequency regime. The step change provides an efficient way to obtain a large 

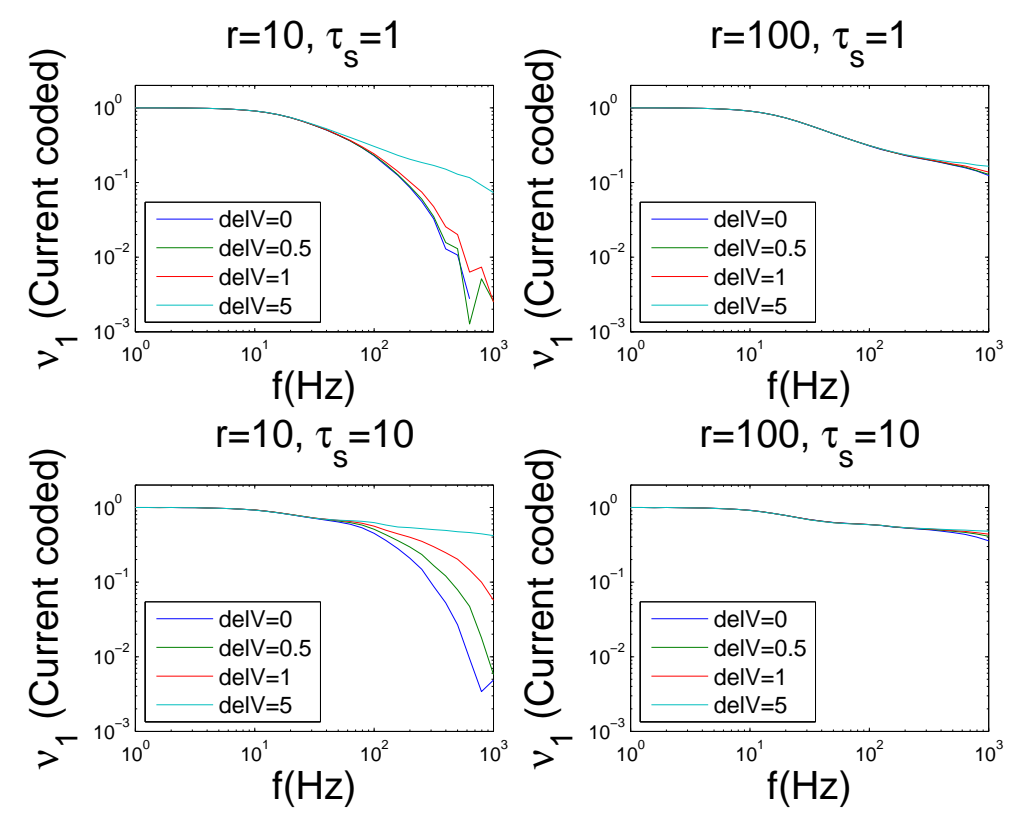

Figure 3.11: Variation of the response $\left|\nu_{1 c}(\omega)\right|$ with increasing $\Delta \dot{v}$ for different $\tau_{s}$ and $r$.
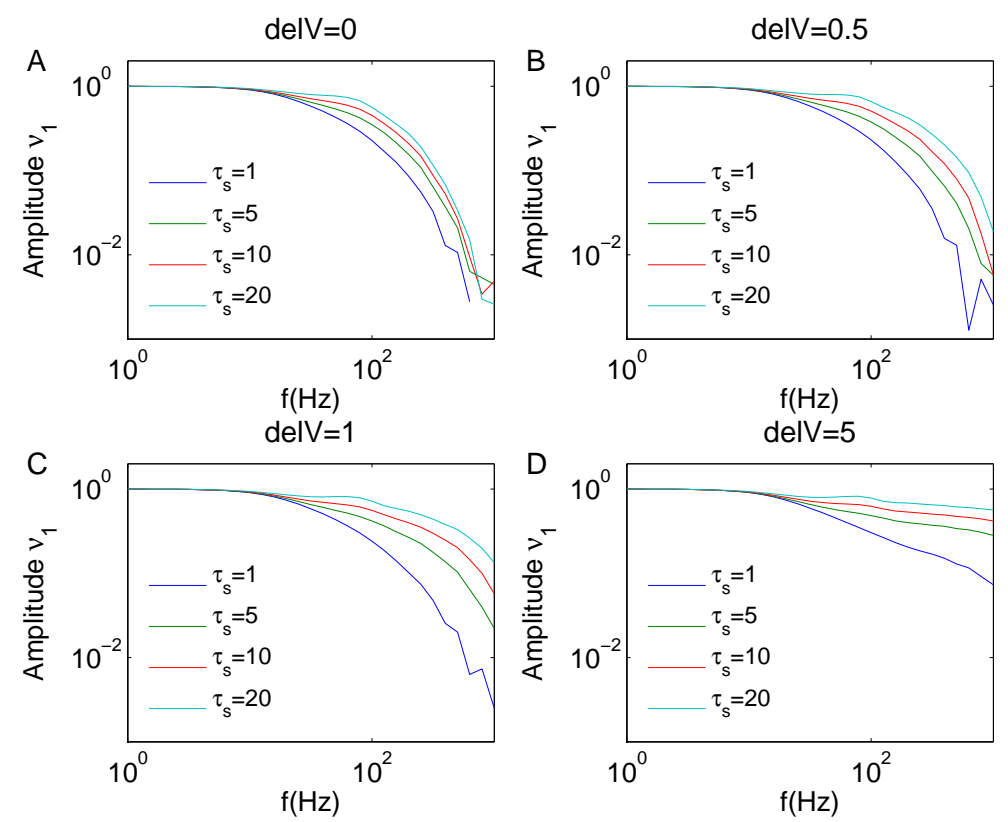

Figure 3.12: Dependence of the transmission function $\left|\nu_{1 c}(\omega)\right|$ on the correlation time $\tau_{s}$ for different $\Delta \dot{v}, r$ is fixed to be 10 . 
onset rapidness. Even for a small sub-threshold step change the cutoff frequency in the colored noise case can reproduce the hundreds HZ cutoff frequency in the linear response, although it has almost no effect on the stationary firing rate and the linear response for the white noise case.

For a colored noise, the artifactual behavior in the response function for high signal frequency appears for small AP onset rapidness, but we found it is suppressed with the increasing of boundary $v_{b}$, with the response function for low and intermediate frequencies not influenced. This suggests that theoretically an isolation of boundary induced artifacts in the colored noise case is also possible. The high frequency limit of the linear response in the LIF model was obtained in (Brunel et al., 2001). In the LIF model, however, the transmission function has no attenuation at high signal frequency resulting from the infinite response speed at the threshold. The results there reflect mainly the contribution from the absorbing boundary. The stationary firing rate of the LIF model for a colored noise has been solved analytically for small or large correlation time $\tau_{s}$, that is, for $\frac{\tau_{s}}{\tau_{m}} \ll 1$ or $\frac{\tau_{s}}{\tau_{m}} \gg 1$ (Brunel et al., 2001). The method might be generalized to the piecewise linear model we have constructed in this thesis, which provides an interesting future direction. 


\section{Chapter 4}

\section{A general piecewise linear model}

In this chapter, we will construct a general piecewise linear model which enables a close relation with the biologically detailed models like the EIF model. This general 3-piece model allows us to study the relation between the details of spike generation and the dynamical response in depth. It includes models presented in previous chapters as limit cases. Mimicking the EIF model with the 2-piece model (Chapter 2) is only qualitative, in the sense that they both include the unstable fixed point for spike initiation. In this new model, we can disassemble the spike generating current into sub-threshold and supra-threshold parts and concentrate on the role of each part played in the dynamical response separately. By checking these aspects carefully, we will give a procedure for finding the parameters of the 3-piece model which can reproduce the stationary and dynamical response of the EIF model very well, and therefore also of conductance based models and presumably even real cortical neurons. The 3-piece model can also be applied to the study of a bistable system. Both the HH model and the two-variable FN model have regimes of bistable dynamics, which was used to model experimental data (Longtin, 1993). Chow \& White (1996) proved that when the fluctuations of sodium channels are included, the dynamics of the $\mathrm{HH}$ model can be approximated by a bistable system and the firing rate is approximated by the barrier penetration rate in a double well, which has be studied using the Kramers rate approximation. In this application of the model, we will provide an analytical result for the linear response of a bistable system.

\subsection{Description of the model}

The 3-piece model is defined by the following Langevin equation

$$
\tau_{m} \dot{v}=f(v)+\mu+\sigma \eta(t)
$$


where

$$
f(v)=\left\{\begin{array}{lrr}
-v, & -\infty<v \leq v_{0} & \text { piece } 1 \\
r_{1}\left(v-v_{t 1}\right), & v_{0}<v \leq v_{1} & \text { piece } 3 \\
r\left(v-v_{t 0}\right), & v_{1}<v \leq v_{b} & \text { piece } 2
\end{array}\right.
$$

Here $v_{0}$ is the crossing point of the left piece (piece 1, the leak current region) with the middle piece (piece 3 ), and $v_{1}$ is the crossing point of the middle piece with the right piece (piece 2). $v_{t 1}$ and $v_{t 0}$ are the zero-crossing points of the middle piece and right piece respectively, given by $v_{t 1}=\left(1+\frac{1}{r_{1}}\right) v_{0}$ and $v_{t 0}=$ $\frac{1}{r}\left(\left(r-r_{1}\right) v_{1}+\left(1+r_{1}\right) v_{0}\right)$, where $r_{1}$ and $r$ are the slopes of the middle piece and the right piece respectively. $r_{1}$ can be positive or negative, with $r_{1}=0$ as a well defined limit case, as illustrated in Fig. 4.1 A, B and C. When the membrane potential reaches an absorbing boundary $v_{b}$, it is reset to a voltage $v_{r}$ and stays there for a refractory period $\tau_{r}$. When $r>0$ (Fig. 4.1A, B and C), the model describes an excitable system for the AP generation process. When $r_{1}$ is positive and $r$ is negative, the model describes a $\mathrm{N}$-shape dynamics for a bistable system, with the two stable fixed point at 0 and $v_{t 0}$, and an unstable fixed point at $v_{t 1}$. We will present a general results for all these cases. A Gaussian white noise $\eta(t)$ will be used for the synaptic noise, which satisfies $\langle\eta(t)\rangle=0$ and $\left\langle\eta(t) \eta\left(t^{\prime}\right)\right\rangle=\tau_{m} \delta\left(t-t^{\prime}\right)$. For the bistable system, an absorbing boundary is put near the stable fixed point $v_{t 0}$ and the dynamics describe the barrier penetration in a double well.

The Fokker-Planck equation corresponding to Eq. (4.1) has the following form

$$
\left\{\begin{array}{l}
\partial_{t} P_{1}(v, t)+\partial_{v}\left(-v+\mu-\frac{1}{2} \sigma^{2} \partial_{v}\right) P_{1}(v, t)=0, \quad-\infty<v \leq v_{0} \\
\partial_{t} P_{3}(v, t)+\partial_{v}\left(r_{1}\left(v-v_{t 1}\right)+\mu-\frac{1}{2} \sigma^{2} \partial_{v}\right) P_{3}(v, t)=0, v_{0}<v \leq v_{1} \\
\partial_{t} P_{2}(v, t)+\partial_{v}\left(r\left(v-v_{t}\right)+\mu-\frac{1}{2} \sigma^{2} \partial_{v}\right) P_{2}(v, t)=0, v_{1}<v \leq v_{b}
\end{array}\right.
$$

where $P_{1}(v, t), P_{3}(v, t)$ and $P_{2}(v, t)$ are the probability densities of membrane potential $v$ with $-\infty<v \leq v_{0}, v_{0}<v \leq v_{1}$ and $v_{1}<v \leq v_{b}$ respectively.

Defining the probability currents by

$$
\begin{aligned}
& J_{1}(v, t)=\left(-v+\mu-\frac{1}{2} \sigma^{2} \partial_{v}\right) P_{1}(v, t) \\
& J_{3}(v, t)=\left(r_{1}\left(v-v_{t 1}\right)+\mu-\frac{1}{2} \sigma^{2} \partial_{v}\right) P_{3}(v, t) \\
& J_{2}(v, t)=\left(r\left(v-v_{t 0}\right)+\mu-\frac{1}{2} \sigma^{2} \partial_{v}\right) P_{2}(v, t)
\end{aligned}
$$


A

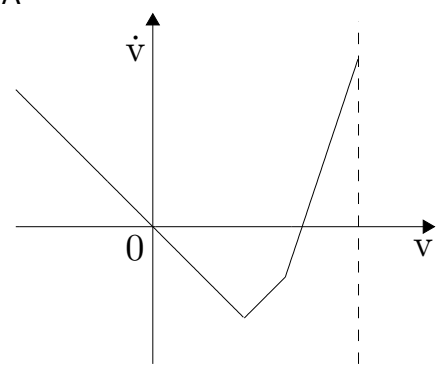

C

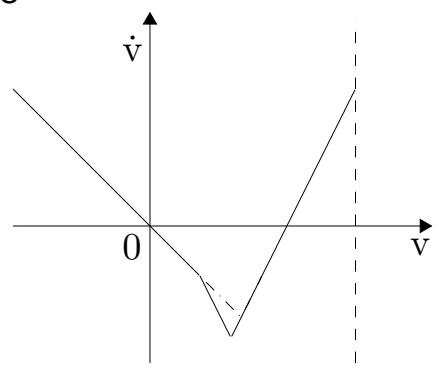

B

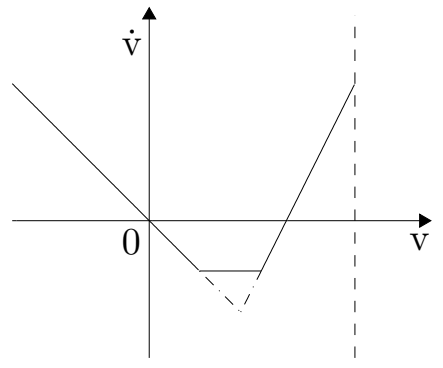

D

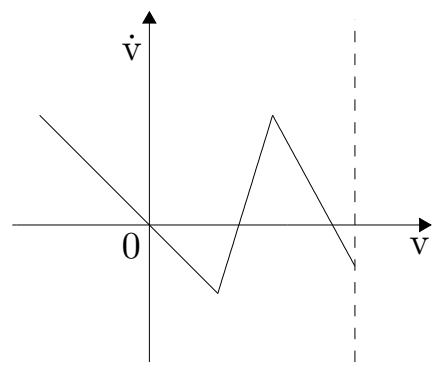

Figure 4.1: Illustration of the model. A, positive $r_{1}, \mathrm{~B}, r_{1}=0$, C, negative $r_{1}$, $\mathrm{D}$, positive $r_{1}$ and negative $r$, a bistable system.

the FPE then becomes the equation for probability conservation,

$$
\partial_{t} P(v, t)+\partial_{v} J(v, t)=0 .
$$

The boundary conditions are the same as described in the previous chapters. At the absorbing boundary $v_{b}$,

$$
P_{2}\left(v_{b}, t\right)=0 .
$$

At the resetting point $v_{r}$

$$
\begin{aligned}
P_{1}\left(v_{r}^{+}, t\right)-P_{1}\left(v_{r}^{-}, t\right) & =0, \\
\partial_{v} P_{1}\left(v_{r}^{+}, t\right)-\partial_{v} P_{1}\left(v_{r}^{-}, t\right) & =\partial_{v} P_{2}\left(v_{b}, t-\tau_{r}\right),
\end{aligned}
$$

from the continuity of the probability density and probability current at $v_{r}$ and the resetting condition. At $v_{0}$ and $v_{1}$, there are

$$
\begin{aligned}
P_{1}\left(v_{0}, t\right) & =P_{3}\left(v_{0}, t\right), \\
\partial_{v} P_{1}\left(v_{0}, t\right) & =\partial_{v} P_{3}\left(v_{0}, t\right), \\
P_{3}\left(v_{1}, t\right) & =P_{2}\left(v_{1}, t\right), \\
\partial_{v} P_{3}\left(v_{1}, t\right) & =\partial_{v} P_{2}\left(v_{1}, t\right) .
\end{aligned}
$$

Finally the normalization condition of the probability density requires that

$$
\lim _{v \rightarrow-\infty} P_{1}(v, t)=0 .
$$


With these boundary conditions the solution of the FPE (4.3) is uniquely determined. The instantaneous firing rate in the population is given by the probability current through the absorbing boundary

$$
\nu(t) \equiv J_{2}\left(v_{b}, t\right)=-\frac{1}{2} \sigma^{2} \partial_{v} P_{2}\left(v_{b}, t\right)
$$

\subsection{Stationary solutions}

When the mean input current $\mu$ and the noise strength $\sigma$ are constants, the system is homogeneous and possesses a stationary solution with a constant probability current, i.e., $J_{1}=J_{3}=J_{2}=\nu_{0}$, where $\nu_{0}$ is the stationary firing rate. We denote the stationary probability densities as $P_{01}(v), P_{03}(v)$ and $P_{02}(v)$ for the three pieces respectively. From the continuity of probability current, there are

$$
J_{1}(v)=J_{3}(v)=J_{2}(v)=\nu_{0},
$$

which is equivalent to

$$
\begin{array}{ll}
\left(-v+\mu-\frac{1}{2} \sigma^{2} \partial_{v}\right) P_{01}(v)=0, & -\infty<v \leq v_{r} \\
\left(-v+\mu-\frac{1}{2} \sigma^{2} \partial_{v}\right) P_{01}(v)=\nu_{0}, & v_{r}<v \leq v_{0} \\
\left(r_{1}\left(v-v_{t 1}\right)+\mu-\frac{1}{2} \sigma^{2} \partial_{v}\right) P_{03}(v)=\nu_{0}, & v_{0}<v \leq v_{1} \\
\left(r\left(v-v_{t 0}\right)+\mu-\frac{1}{2} \sigma^{2} \partial_{v}\right) P_{02}(v)=\nu_{0}, & v_{1}<v \leq v_{b}
\end{array}
$$

Utilizing the boundary conditions Eq. (4.6-4.10), above equations are solved,

$$
\begin{aligned}
P_{01}(v)= & \frac{2 \nu_{0}}{\sigma^{2}} e^{-\frac{1}{\sigma^{2}}\left(v^{2}-2 \mu v\right)}\left(\int_{\max \left(v, v_{r}\right)}^{v_{0}} e^{\frac{1}{\sigma^{2}}\left(y^{2}-2 \mu y\right)} d y\right. \\
+ & \left.e^{A} \int_{v_{0}}^{v_{1}} e^{-\frac{1}{\sigma^{2}}\left(r_{1} y^{2}-2\left(r_{1} v_{t 1}-\mu\right) y\right)} d y+e^{A+B} \int_{v_{1}}^{v_{b}} e^{-\frac{1}{\sigma^{2}}\left(r y^{2}-2\left(r v_{t}-\mu\right) y\right)} d y\right) \\
P_{03}(v)= & \frac{2 \nu_{0}}{\sigma^{2}} e^{\frac{1}{\sigma^{2}}\left(r_{1} v^{2}-2\left(r_{1} v_{t 1}-\mu\right) v\right)}\left(\int_{v}^{v_{1}} e^{-\frac{1}{\sigma^{2}}\left(r_{1} y^{2}-2\left(r_{1} v_{t 0}-\mu\right) y\right)} d y\right. \\
& \left.+e^{B} \int_{v_{1}}^{v_{b}} e^{-\frac{1}{\sigma^{2}}\left(r y^{2}-2\left(r v_{t}-\mu\right) y\right)} d y\right) \\
P_{02}(v)= & \frac{2 \nu_{0}}{\sigma^{2}} e^{\frac{1}{\sigma^{2}}\left(r v^{2}-2\left(r v_{t 0}-\mu\right) v\right)} \int_{v}^{v_{b}} e^{-\frac{1}{\sigma^{2}}\left(r y^{2}-2\left(r v_{t 0}-\mu\right) y\right)} d y
\end{aligned}
$$


where

$$
\begin{aligned}
A & =\frac{1}{\sigma^{2}}\left(v_{0}^{2}-2 \mu v_{0}+r_{1} v_{0}^{2}-2\left(r_{1} v_{t 1}-\mu\right) v_{0}\right) \\
& =-\frac{1}{\sigma^{2}}\left(1+r_{1}\right) v_{0}^{2} \\
B & =\frac{1}{\sigma^{2}}\left(r v_{1}^{2}-2\left(r v_{t 0}-\mu\right) v_{1}-\left(r_{1} v_{1}^{2}-2\left(r_{1} v_{t 1}-\mu\right) v_{1}\right)\right) \\
& =-\frac{1}{\sigma^{2}}\left(r-r_{1}\right) v_{1}^{2} .
\end{aligned}
$$

This can be further simplified after the signs of $r_{1}$ and $r$ are assigned and the stationary firing rate $\nu_{0}$ is obtained from the normalization condition,

$$
\int_{-\infty}^{v_{b}} P_{0}(v) d v=1
$$

which are presented in the Appendix.

\subsection{Linear responses}

\subsubsection{Linear response to a current coded signal}

Consider a weak sinusoidal signal encoded in the mean input, $\mu(t)=\mu+\varepsilon \cos (\omega t)$, where $\varepsilon$ is small. At the linear order, the probability densities can be expanded as

$$
\begin{aligned}
& P_{1}(v, t)=P_{01}(v)+\varepsilon P_{11}(v, t)+\cdots, \\
& P_{3}(v, t)=P_{03}(v)+\varepsilon P_{13}(v, t)+\cdots, \\
& P_{2}(v, t)=P_{02}(v)+\varepsilon P_{12}(v, t)+\cdots,
\end{aligned}
$$

where '...' represents higher order terms in $\varepsilon$. The Fokker-Planck equation at the linear order in $\varepsilon$ is then given by

$\partial_{t} P_{11}(v, t)=\partial_{v}\left(v-\mu+D \partial_{v}\right) P_{11}(v, t)-\cos (\omega t) P_{01}^{\prime}(v), \quad-\infty<v \leq v_{0}$
$\partial_{t} P_{13}(v, t)=\partial_{v}\left(-r_{1} v+r_{1} v_{t 1}-\mu+D \partial_{v}\right) P_{13}(v, t)-\cos (\omega t) P_{03}^{\prime}(v), v_{0}<v \leq v_{1}$
$\partial_{t} P_{12}(v, t)=\partial_{v}\left(-r v+r v_{t 0}-\mu+D \partial_{v}\right) P_{12}(v, t)-\cos (\omega t) P_{02}^{\prime}(v), \quad v_{0}<v \leq v_{b}$

where $D=\frac{1}{2} \sigma^{2}$ is the diffusion constant.

Factoring $P_{11}(v, t), P_{13}(v, t)$ and $P_{12}(v, t)$ as follows

$$
\begin{aligned}
& P_{11}(v, t)=\frac{1}{2}\left(e^{-i \omega t} q_{1}(v)+e^{i \omega t} q_{1}^{*}(v)\right) e^{-(v-\mu)^{2} / 4 D}, \\
& P_{13}(v, t)=\frac{1}{2}\left(e^{-i \omega t} q_{3}(v)+e^{i \omega t} q_{3}^{*}(v)\right) e^{\left(r_{1} v^{2}-2\left(r_{1} v_{t}-\mu\right) v\right) / 4 D}, \\
& P_{12}(v, t)=\frac{1}{2}\left(e^{-i \omega t} q_{2}(v)+e^{i \omega t} q_{2}^{*}(v)\right) e^{\left(r v^{2}-2\left(r v_{t 0}-\mu\right) v\right) / 4 D},
\end{aligned}
$$


where the asterisks represent complex conjugate, we obtain

$$
\begin{aligned}
& D q_{1}^{\prime \prime}(v)-\left(\frac{(v-\mu)^{2}}{4 D}-i \omega-\frac{1}{2}\right) q_{1}(v)=e^{(v-\mu)^{2} / 4 D} P_{01}^{\prime}(v) \\
& D q_{3}^{\prime \prime}(v)-\left(\frac{\left(r_{1}\left(v-v_{t 1}\right)+\mu\right)^{2}}{4 D}-i \omega+\frac{r_{1}}{2}\right) q_{3}(v)=e^{-\left(r_{1} v^{2}-2\left(r_{1} v_{t 1}-\mu\right) v\right) / 4 D} P_{03}^{\prime}(v) \\
& \frac{D}{r} q_{2}^{\prime \prime}(v)-\left(\frac{\left(v-v_{t 0}+\mu / r\right)^{2}}{4 D / r}-\frac{i \omega}{r}+\frac{1}{2}\right) q_{2}(v)=\frac{1}{r} e^{-\left(r v^{2}-2\left(r v_{t 0}-\mu\right) v\right) / 4 D} P_{02}^{\prime}(v)
\end{aligned}
$$

where in the equation for $q_{3}(v)$, we did not divide $r_{1}$ from both sides, since we will also study the case $r_{1}=0$. When $r_{1} \neq 0, q_{3}(v)$ shares a similar equation to $q_{2}(v)$. Substituting Eq. (4.17) and Eq. (4.19) into the boundary conditions Eq. (4.6 4.10), $q_{1}, q_{3}$ and $q_{2}$ satisfy the following boundary conditions,

$$
\begin{aligned}
\lim _{v \rightarrow-\infty} q_{1}(v) & =0, \\
q_{2}\left(v_{b}\right) & =0 \\
q_{1}\left(v_{0}\right) & =q_{3}\left(v_{0}\right) e^{\Delta_{02}}, \\
q_{1}^{\prime}\left(v_{0}\right) & =q_{3}^{\prime}\left(v_{0}\right) e^{\Delta_{02}}, \\
q_{3}\left(v_{1}\right) & =q_{2}\left(v_{1}\right) e^{\Delta_{03}}, \\
q_{3}^{\prime}\left(v_{1}\right) & =q_{3}^{\prime}\left(v_{1}\right) e^{\Delta_{03}}, \\
q_{1}\left(v_{r}^{+}\right)-q_{1}\left(v_{r}^{-}\right) & =0 \\
q_{1}^{\prime}\left(v_{r}^{+}\right)-q_{1}^{\prime}\left(v_{r}^{-}\right) & =q_{3}^{\prime}\left(v_{b}\right) e^{\Delta_{01}+i \omega \tau_{r}},
\end{aligned}
$$

where

$$
\begin{aligned}
& \Delta_{01}=\left(\left(v_{r}-\mu\right)^{2}+\left(r v_{b}^{2}-2\left(r v_{t 0}-\mu\right) v_{b}\right)\right) / 4 D \\
& \Delta_{02}=\left(\left(v_{0}-\mu\right)^{2}+\left(r_{1} v_{0}^{2}-2\left(r_{1} v_{t 1}-\mu\right) v_{0}\right)\right) / 4 D, \\
& \Delta_{03}=\left(\left(r v_{1}^{2}-2\left(r v_{t 0}-\mu\right) v_{1}\right)-\left(r_{1} v_{1}^{2}-2\left(r_{1} v_{t 1}-\mu\right) v_{1}\right)\right) / 4 D .
\end{aligned}
$$

Eq. (4.20) can be solved using the Green's function method. The homogeneous parts of Eq. (4.20) have the form of Weber' equation whose solutions are the parabolic cylinder functions (Abramowitz \& Stegun, 1970). The homogeneous equation for $q_{1}(v)$ keeps unchanged for the different models we are discussing, as illustrated in Fig. 4.1. Its solution is expressed with the parabolic cylinder functions defined in Chapter 2. When $r>0$, the parabolic cylinder functions used for piece 2 are also given in Chapter 2. But different choice for $r_{1}$ and $r$ with positive or negative sign did make the Weber's equations different. For example, when $r_{1}<0$, the equation for $q_{3}$ needs to be rewritten as

$$
\begin{aligned}
& \frac{D}{\left|r_{1}\right|} q_{3}^{\prime \prime}(v)-\left(\frac{\left(v-v_{t 1}+\mu / r_{1}\right)^{2}}{4 D /\left|r_{1}\right|}+\frac{i \omega}{r_{1}}-\frac{1}{2}\right) q_{3}(v) \\
& =\frac{1}{\left|r_{1}\right|} e^{-\left(r_{1} v^{2}-2\left(r_{1} v_{t 1}-\mu\right) v\right) / 4 D} P_{03}^{\prime}(v)
\end{aligned}
$$


and the parabolic cylinder functions as the solution of the homogeneous equation for $q_{3}(v)$ are different from the positive $r_{1}$ case. Similar is for the $r<0$ case. With the parabolic cylinder functions as defined in the Appendix, the Green's function can be constructed and the solutions for the inhomogeneous equations can be written down. Similar to that in Chapter 2, the Green's functions are

$$
\begin{aligned}
& g_{1}(v, y)=-\frac{1}{D} \begin{cases}\psi_{2}(y) \psi_{1}(v), & v<y \\
\psi_{1}(y) \psi_{2}(v), & y \leq v<v_{0}\end{cases} \\
& g_{3}(v, y)=\frac{\left|r_{1}\right|}{\psi_{5}\left(v_{1}\right) D} \begin{cases}\psi_{5}(y) \psi_{6}(v), & v_{0} \leq v<y \\
\psi_{5}(v) \psi_{6}(y), & y \leq v<v_{1}\end{cases} \\
& g_{2}(v, y)=\frac{|r|}{\psi_{3}\left(v_{b}\right) D} \begin{cases}Y_{1}(y) \psi_{3}(v), & v_{1} \leq v<y \\
\psi_{3}(y) Y_{1}(v), & y \leq v<v_{b}\end{cases}
\end{aligned}
$$

which satisfy the boundary conditions Eq. (4.21) and Eq. (4.22). The solutions of Eq. (4.20) are given by

$$
\begin{aligned}
q_{1}(v)= & -\frac{1}{D}\left(\psi_{2}(v) \int_{-\infty}^{v} \psi_{1}(y) P_{01}^{\prime} e^{(y-\mu)^{2} / 4 D} d y\right. \\
& \left.+\psi_{1}(v) \int_{v}^{v_{0}} \psi_{2}(y) P_{01}^{\prime} e^{(y-\mu)^{2} / 4 D} d y\right) \\
& +k_{1}\left\{\begin{array}{l}
\psi_{2}\left(v_{r}\right) \psi_{1}(v), \quad v<v_{r} \\
\psi_{1}\left(v_{r}\right) \psi_{2}(v)+a\left(\psi_{2}\left(v_{r}\right) \psi_{1}(v)-\psi_{1}\left(v_{r}\right) \psi_{2}(v)\right), \quad v_{r} \leq v<v_{0}
\end{array}\right. \\
q_{3}(v)= & \frac{1}{D}\left(\psi_{5}(v) \int_{v_{0}}^{v} \psi_{6}(y) P_{03}^{\prime}(y) e^{-\left(r_{1} y^{2}-2\left(r_{1} v_{t 1}-\mu\right) y\right) / 4 D} d y\right. \\
& \left.+\psi_{6}(v) \int_{v}^{v_{1}} \psi_{5}(y) P_{03}^{\prime}(y) e^{-\left(r_{1} y^{2}-2\left(r_{1} v_{t 1}-\mu\right) y\right) / 4 D} d y\right) \\
& +b \psi_{5}(v)+c \psi_{6}(v), \quad v_{0}<v \leq v_{1} \\
& \frac{1}{q_{3}(v) D}\left(v_{b}(v) \int_{v_{1}}^{v} \psi_{3}(y) P_{02}^{\prime}(y) e^{-\left(r y^{2}-2\left(r v_{t}-\mu\right) y\right) / 4 D} d y\right. \\
& \left.+\psi_{3}(v) \int_{v}^{v_{b}} Y_{1}(y) P_{02}^{\prime}(y) e^{-\left(r y^{2}-2\left(r v_{t}-\mu\right) y\right) / 4 D} d y\right) \\
& +k_{2} Y_{1}(v), \quad v_{0}<v \leq v_{b}
\end{aligned}
$$

$k_{1}, k_{2}, a, b$ and $c$ are free parameters and will be fixed by the boundary conditions (4.23,4.28). After some algebra, we then get

$$
\begin{aligned}
q_{2}^{\prime}\left(v_{b}\right)= & -\frac{1}{D} e^{-\Delta_{01}-i \omega \tau_{r}}(A+B+G)\left(\psi_{1}\left(v_{r}\right)\right. \\
& \left.-\left(Y_{1}\left(v_{1}\right)\left(\psi_{1} Y_{6}^{\prime}-\psi_{1}^{\prime} Y_{6}\right)-Y_{1}^{\prime}\left(v_{1}\right)\left(\psi_{1} Y_{5}^{\prime}-\psi_{1}^{\prime} Y_{5}\right)\right) e^{\Delta_{02}+\Delta_{03}-\Delta_{01}-i \omega \tau_{r}}\right)^{-1}
\end{aligned}
$$


with

$$
\begin{aligned}
A= & \int_{-\infty}^{v_{0}} \psi_{1}(y) P_{01}^{\prime}(y) e^{(y-\mu)^{2} / 2 \sigma^{2}} d y \\
B= & \left.\frac{e^{\Delta_{02}+\Delta_{03}}}{\psi_{3}\left(v_{b}\right)} \int_{v_{1}}^{v_{b}}\left(\left(\psi_{1} Y_{5}^{\prime}-\psi_{1}^{\prime} Y_{5}\right) \psi_{3}^{\prime}\left(v_{1}\right)-\psi_{1} Y_{6}^{\prime}-\psi_{1}^{\prime} Y_{6}\right) \psi_{3}\left(v_{1}\right)\right) Y_{1}(y) \\
& \left.\left.\left.-\left(\psi_{1} Y_{5}^{\prime}-\psi_{1}^{\prime} Y_{5}\right) Y_{1}^{\prime}\left(v_{1}\right)-\psi_{1} Y_{6}^{\prime}-\psi_{1}^{\prime} Y_{6}\right) Y_{1}\left(v_{1}\right)\right) \psi_{3}(y)\right) \\
& \times P_{02}^{\prime}(y) e^{-\left(r y^{2}-\left(r v_{t 0}-\mu\right) y\right) / 4 D} d y \\
G= & e^{\Delta_{02}} \int_{v_{0}}^{v_{1}}\left(\left(\psi_{1} Y_{6}^{\prime}-\psi_{1}^{\prime} Y_{6}\right) Y_{5}(y)-\left(\psi_{1} Y_{5}^{\prime}-\psi_{1}^{\prime} Y_{5}\right) Y_{6}(y)\right) \\
& \times P_{03}^{\prime}(y) e^{-\left(r_{1} y^{2}-\left(r_{1} v_{t 1}-\mu\right) y\right) / 4 D} d y
\end{aligned}
$$

Following the convention in previous chapters, the arguments of the parabolic cylinder functions and of the probability density are taken at $v_{0}$ unless denoted explicitly.

At the linear order in $\varepsilon$, the instantaneous firing rate is given by

$$
\begin{aligned}
\nu(t) & =-D \partial_{v} P\left(v_{b}, t\right) \\
& =\nu_{0}-\frac{\varepsilon}{2} D\left(e^{-i \omega t} q_{2}^{\prime}\left(v_{b}\right)+e^{i \omega t} q_{2}^{\prime}\left(v_{b}\right)\right) e^{\left(r v_{b}^{2}-2\left(r v_{t 0}-\mu\right) v_{b}\right) / 4 D} \\
& =\nu_{0}+\varepsilon\left|\nu_{1 c}(\omega)\right| \cos \left(\omega t-\phi_{c}(\omega)\right),
\end{aligned}
$$

where

$$
\nu_{1 c}(\omega)=-D q_{2}^{\prime}\left(v_{b}\right) e^{\left(r v_{b}^{2}-2\left(r v_{t 0}-\mu\right) v_{b}\right) / 4 D}
$$

is the complex response function.

The integrals in Eq. (4.33) can be performed as outlined in Chapter 2. Note, however, that since the definitions of the parabolic cylinder functions as the solutions of homogeneous parts in Eq. (4.20) are different for positive and negative signs of $r_{1}$ and $r$, the derived functions encountered in performing the integral, defined through applying the recurrence relations for the parabolic cylinder functions, are different for different signs in piece 2 and piece 3 . For example, in performing the integral in Eq. (4.36), we need to define the following functions, $\phi_{5}(v), \psi_{6}(v)$, and $Y_{51}(v), Y_{52}(v)$, which are different for positive and negative $r_{1}$. So we need to calculate for the three cases (corresponding to A, C and D in Fig. 4.1) separately.

A good thing is that the linear responses share the same finial expressions for three cases. We find that the expression for $\nu_{1 c}(\omega)$ can be decomposed into two parts,

$$
\nu_{1 c}(\omega)=\nu_{1 c}^{L o w}(\omega)+\nu_{1 c}^{H i g h}(\omega)
$$


with

$$
\begin{aligned}
\nu_{1 c}^{\text {Low }}(\omega)= & \left(\psi_{1}\left(v_{r}\right) e^{\Delta_{0}+i \omega \tau_{r}}+\left(\left(\psi_{1} Y_{5}^{\prime}-\psi_{1}^{\prime} Y_{5}\right) Y_{1}^{\prime}-\left(\psi_{1} Y_{6}^{\prime}-\psi_{1}^{\prime} Y_{6}\right) Y_{1}\right) e^{\Delta_{7}}\right)^{-1} \\
& \left(\left(\frac{1}{1-i \omega}-\frac{1}{1+i \omega / r_{1}}\right)\left(\psi_{1} P_{01}-\sqrt{D} \Phi_{1} P_{01}^{\prime}\right)\right. \\
& -\frac{\nu_{0}}{\sqrt{D}} \frac{i \omega}{1-i \omega} \Phi_{1}\left(v_{r}\right) e^{\Delta_{0}} \\
& +\left(\frac{1}{1+i \omega / r}-\frac{1}{1+i \omega / r_{1}}\right)\left(\psi_{1} Y_{5}^{\prime}-\psi_{1}^{\prime} Y_{5}\right) P_{02}\left(v_{1}\right) e^{\Delta_{6}} \\
& \left.+\sqrt{D\left|r_{1}\right|}\left(\frac{1}{r_{1}} \frac{1}{1+i \omega / r_{1}}-\frac{1}{r} \frac{1}{1+i \omega / r}\right)\left(\psi_{1} Y_{51}^{\prime}-\psi_{1}^{\prime} Y_{51}\right) P_{02}^{\prime}\left(v_{1}\right) e^{\Delta_{6}}\right) \\
\nu_{1 c}^{\text {High }}(\omega)= & \left(\psi_{1}\left(v_{r}\right) e^{\Delta_{0}+i \omega \tau_{r}}+\left(\left(\psi_{1} Y_{5}^{\prime}-\psi_{1}^{\prime} Y_{5}\right) Y_{1}^{\prime}-\left(\psi_{1} Y_{6}^{\prime}-\psi_{1}^{\prime} Y_{6}\right) Y_{1}\right) e^{\Delta_{7}}\right)^{-1} \\
& \times \frac{1}{1+i \omega / r} \frac{\nu_{0}}{\sqrt{|r| D}}\left(\left(\psi_{1} Y_{5}^{\prime}-\psi_{1}^{\prime} Y_{5}\right) Y_{2}^{\prime}-\left(\psi_{1} Y_{6}^{\prime}-\psi_{1}^{\prime} Y_{6}\right) Y_{2}\right) e^{\Delta_{7}} .
\end{aligned}
$$

where $\Delta_{0}=\left(\left(v_{r}-\mu\right)^{2}-\left(v_{0}-\mu\right)^{2}\right) / 4 D$ is the same as in Chapter 2 and

$$
\begin{aligned}
\Delta_{6}= & \left(r_{1} v_{0}^{2}-2\left(r_{1} v_{t 1}-\mu\right) v_{0}\right)-\left(r_{1} v_{1}^{2}-2\left(r_{1} v_{t 1}-\mu\right) v_{1}\right) / 4 D \\
\Delta_{7}= & \left(\left(r_{1} v_{0}^{2}-2\left(r_{1} v_{t 1}-\mu\right) v_{0}\right)-\left(r_{1} v_{1}^{2}-2\left(r_{1} v_{t 1}-\mu\right) v_{1}\right)\right. \\
& \left.+\left(r v_{1}^{2}-2\left(r v_{t 0}-\mu\right) v_{1}\right)-\left(r v_{b}^{2}-2\left(r v_{t 0}-\mu\right) v_{b}\right)\right) / 4 D
\end{aligned}
$$

\subsubsection{Linear response to a noise coded signal}

For a noise coded signal, we have $\sigma(t)=\sigma+\varepsilon \cos (\omega t)$. Similar to the case of a current coded signal, the probability densities can be divided into

$$
\begin{aligned}
& P_{1}(v, t)=P_{01}(v)+\varepsilon \sigma P_{21}(v, t)+\cdots \\
& P_{3}(v, t)=P_{03}(v)+\varepsilon \sigma P_{23}(v, t)+\cdots \\
& P_{2}(v, t)=P_{02}(v)+\varepsilon \sigma P_{22}(v, t)+\cdots
\end{aligned}
$$

and the FPE becomes

$$
\begin{aligned}
& \partial_{t} P_{21}(v, t)=\partial_{v}\left(v-\mu+D \partial_{v}\right) P_{21}(v, t)+\cos (\omega t) P_{01}^{\prime \prime}(v), \quad-\infty<v \leq v_{0} \\
& \partial_{t} P_{23}(v, t)=\partial_{v}\left(-r_{1} v+r_{1} v_{t 1}-\mu+D \partial_{v}\right) P_{23}(v, t)+\cos (\omega t) P_{03}^{\prime \prime}(v), v_{0}<v \leq v_{1} \\
& \partial_{t} P_{22}(v, t)=\partial_{v}\left(-r v+r v_{t 0}-\mu+D \partial_{v}\right) P_{22}(v, t)+\cos (\omega t) P_{02}^{\prime \prime}(v), \quad v_{0}<v \leq v_{b}
\end{aligned}
$$


Factoring the densities as

$$
\begin{aligned}
& P_{21}(v, t)=\frac{1}{2}\left(e^{-i \omega t} q_{1}(v)+e^{i \omega t} q_{1}^{*}(v)\right) e^{-(v-\mu)^{2} / 4 D}, \\
& P_{23}(v, t)=\frac{1}{2}\left(e^{-i \omega t} q_{3}(v)+e^{i \omega t} q_{3}^{*}(v)\right) e^{\left(r_{1} v^{2}-2\left(r_{1} v_{t 1}-\mu\right) v\right) / 4 D}, \\
& P_{22}(v, t)=\frac{1}{2}\left(e^{-i \omega t} q_{2}(v)+e^{i \omega t} q_{2}^{*}(v)\right) e^{\left(r v^{2}-2\left(r v_{t 0}-\mu\right) v\right) / 4 D},
\end{aligned}
$$

we then get the following equations

$$
\begin{aligned}
& D q_{1}^{\prime \prime}(v)-\left(\frac{(v-\mu)^{2}}{4 D}-i \omega-\frac{1}{2}\right) q_{1}(v)=-e^{(v-\mu)^{2} / 4 D} P_{01}^{\prime \prime}(v) \\
& D q_{3}^{\prime \prime}(v)-\left(\frac{\left(r_{1}\left(v-v_{t 1}\right)+\mu\right)^{2}}{4 D}-i \omega+\frac{r_{1}}{2}\right) q_{3}(v)=-e^{-\left(r_{1} v^{2}-2\left(r_{1} v_{t 1}-\mu\right) v\right) / 4 D} P_{03}^{\prime \prime}(v) \\
& \frac{D}{r} q_{2}^{\prime \prime}(v)-\left(\frac{\left(v-v_{t 0}+\mu / r\right)^{2}}{4 D / r}-\frac{i \omega}{r}+\frac{1}{2}\right) q_{2}(v)=-\frac{1}{r} e^{-\left(r v^{2}-2\left(r v_{t 0}-\mu\right) v\right) / 4 D} P_{02}^{\prime \prime}(v)
\end{aligned}
$$

The left sides of Eq. (4.45) have the same forms as Eq. (4.20), therefore the definitions of the parabolic cylinder functions and the Green's functions are the same as that for a current coded signal. The differences are from the source terms on the right sides. The solutions of Eq. (4.45) are given by

$$
\begin{aligned}
q_{1}(v)= & \frac{1}{D}\left(\psi_{2}(v) \int_{-\infty}^{v} \psi_{1}(y) P_{01}^{\prime \prime} e^{(y-\mu)^{2} / 4 D} d y\right. \\
& \left.\quad+\psi_{1}(v) \int_{v}^{v_{0}} \psi_{2}(y) P_{01}^{\prime \prime} e^{(y-\mu)^{2} / 4 D} d y\right) \\
& +k_{1}\left\{\begin{array}{l}
\psi_{2}\left(v_{r}\right) \psi_{1}(v), \quad v<v_{r} \\
\psi_{1}\left(v_{r}\right) \psi_{2}(v)+a\left(\psi_{2}\left(v_{r}\right) \psi_{1}(v)-\psi_{1}\left(v_{r}\right) \psi_{2}(v)\right), \quad v_{r} \leq v<v_{0}
\end{array}\right. \\
q_{3}(v)= & \frac{-1}{D}\left(\psi_{5}(v) \int_{v_{0}}^{v} \psi_{6}(y) P_{03}^{\prime \prime}(y) e^{-\left(r_{1} y^{2}-2\left(r_{1} v_{t 1}-\mu\right) y\right) / 4 D} d y\right. \\
& \left.+\psi_{6}(v) \int_{v}^{v_{1}} \psi_{5}(y) P_{03}^{\prime \prime}(y) e^{-\left(r_{1} y^{2}-2\left(r_{1} v_{t 1}-\mu\right) y\right) / 4 D} d y\right) \\
& +b \psi_{5}(v)+c \psi_{6}(v), v_{0}<v \leq v_{1} \\
& \quad-1 \\
& \psi_{3}\left(v_{b}\right) D\left(Y_{1}(v) \int_{v_{1}}^{v} \psi_{3}(y) P_{02}^{\prime \prime}(y) e^{-\left(r y^{2}-2\left(r v_{t 0}-\mu\right) y\right) / 4 D} d y\right. \\
& \left.+\psi_{3}(v) \int_{v}^{v_{b}} Y_{1}(y) P_{02}^{\prime \prime}(y) e^{-\left(r y^{2}-2\left(r v_{t 0}-\mu\right) y\right) / 4 D} d y\right) \\
& +k_{2} Y_{1}(v), v_{0}<v \leq v_{b}
\end{aligned}
$$

$k_{1}, k_{2}, a, b$ and $c$ are free parameters and will be fixed by the boundary conditions (4.23 4.28). 
The instantaneous firing rate is

$$
\begin{aligned}
\nu(t) & =-\left.(D+\varepsilon \sigma \cos (\omega t)) \partial_{v}\left(P_{02}+\varepsilon \sigma P_{22}\right)\right|_{v_{b}} \\
& =\nu_{0}+\varepsilon \sigma\left|\nu_{1 n}(\omega)\right| \cos \left(\omega t-\phi_{n}(\omega)\right),
\end{aligned}
$$

where $\nu_{1 n}(\omega)$ is the complex response function given by

$$
\begin{aligned}
\nu_{1 n}(\omega)= & \frac{\nu_{0}}{D}-D q_{2}^{\prime}\left(v_{b}\right) e^{\left(r v_{b}^{2}-2\left(r v_{t 0}-\mu\right) v_{b}\right) / 4 D} \\
= & \left(\psi_{1}\left(v_{r}\right)-\left(Y_{1}\left(v_{1}\right)\left(\psi_{1} Y_{6}^{\prime}-\psi_{1}^{\prime} Y_{6}\right)\right.\right. \\
& \left.\left.\quad-Y_{1}^{\prime}\left(v_{1}\right)\left(\psi_{1} Y_{5}^{\prime}-\psi_{1}^{\prime} Y_{5}\right)\right) e^{\Delta_{02}+\Delta_{03}-\Delta_{01}-i \omega \tau_{r}}\right)^{-1} \\
& \times\left(e^{-\left(v_{r}-\mu\right)^{2} / 4 D}(-A+B-F)-\frac{\nu_{0}}{D}\left(Y_{1}\left(v_{1}\right)\left(\psi_{1} Y_{6}^{\prime}-\psi_{1}^{\prime} Y_{6}\right)\right.\right. \\
& \left.\left.\quad-Y_{1}^{\prime}\left(v_{1}\right)\left(\psi_{1} Y_{5}^{\prime}-\psi_{1}^{\prime} Y_{5}\right)\right) e^{\Delta_{02}+\Delta_{03}-\Delta_{01}-i \omega \tau_{r}}\right)
\end{aligned}
$$

with

$$
\begin{aligned}
A= & \int_{-\infty}^{v_{0}} \psi_{1}(y) P_{01}^{\prime \prime}(y) e^{(y-\mu)^{2} / 2 \sigma^{2}} d y \\
B= & \left.\frac{e^{\Delta_{02}+\Delta_{03}}}{\psi_{3}\left(v_{b}\right)} \int_{v_{1}}^{v_{b}}\left(\left(\psi_{1} Y_{5}^{\prime}-\psi_{1}^{\prime} Y_{5}\right) \psi_{3}^{\prime}\left(v_{1}\right)-\psi_{1} Y_{6}^{\prime}-\psi_{1}^{\prime} Y_{6}\right) \psi_{3}\left(v_{1}\right)\right) Y_{1}(y) \\
& \left.\left.\left.-\left(\psi_{1} Y_{5}^{\prime}-\psi_{1}^{\prime} Y_{5}\right) Y_{1}^{\prime}\left(v_{1}\right)-\psi_{1} Y_{6}^{\prime}-\psi_{1}^{\prime} Y_{6}\right) Y_{1}\left(v_{1}\right)\right) \psi_{3}(y)\right) \\
& \times P_{02}^{\prime \prime}(y) e^{-\left(r y^{2}-\left(r v_{t 0}-\mu\right) y\right) / 4 D} d y \\
F= & e^{\Delta_{02}} \int_{v_{0}}^{v_{1}}\left(\left(\psi_{1} Y_{6}^{\prime}-\psi_{1}^{\prime} Y_{6}\right) Y_{5}(y)-\left(\psi_{1} Y_{5}^{\prime}-\psi_{1}^{\prime} Y_{5}\right) Y_{6}(y)\right) \\
& \times P_{03}^{\prime \prime}(y) e^{-\left(r_{1} y^{2}-\left(r_{1} v_{t 1}-\mu\right) y\right) / 4 D} d y
\end{aligned}
$$

and $\phi_{n}(\omega)=\arg \left(\nu_{1 n}(\omega)\right)$ is the phase lag.

After performing the integration in Eq. (4.48), we find $\nu_{1 n}(\omega)$ is decomposed into two parts,

$$
\nu_{1 n}(\omega)=\nu_{1 n}^{L o w}(\omega)+\nu_{1 n}^{H i g h}(\omega)
$$


with

$$
\begin{aligned}
\nu_{1 n}^{\text {Low }}(\omega)= & \left(\psi_{1}\left(v_{r}\right) e^{\Delta_{0}+i \omega \tau_{r}}+\left(\left(\psi_{1} Y_{5}^{\prime}-\psi_{1}^{\prime} Y_{5}\right) Y_{1}^{\prime}-\left(\psi_{1} Y_{6}^{\prime}-\psi_{1}^{\prime} Y_{6}\right) Y_{1}\right) e^{\Delta_{7}}\right)^{-1} \\
\times & \left(\left(\frac{1}{2-i \omega}-\frac{1}{2+i \omega / r_{1}}\right)\left(-\frac{i \omega}{\sqrt{D}} \phi_{1} P_{01}+2(i \omega-1) \Upsilon_{1} P_{01}^{\prime}\right)\right. \\
+ & \frac{\nu_{0}}{D} \frac{i \omega(i \omega-1)}{2-i \omega} \Upsilon_{1}\left(v_{r}\right) e^{\Delta_{0}} \\
& +\left(\frac{1}{2+i \omega / r}-\frac{1}{2+i \omega / r_{1}}\right) \frac{\sqrt{\left|r_{1}\right|}}{\sqrt{D}}\left(\psi_{1} Y_{51}^{\prime}-\psi_{1}^{\prime} Y_{51}\right) P_{02}\left(v_{1}\right) e^{\Delta_{6}} \\
& \left.+\left(\frac{1}{2+i \omega / r_{1}}-\frac{r_{1}}{r} \frac{1}{2+i \omega / r}\right)\left(\psi_{1} Y_{52}^{\prime}-\psi_{1}^{\prime} Y_{52}\right) P_{02}^{\prime}\left(v_{1}\right) e^{\Delta_{6}}\right) \\
\nu_{1 n}^{\text {High }}(\omega)= & \left(\psi_{1}\left(v_{r}\right) e^{\Delta_{0}+i \omega \tau_{r}}+\left(\left(\psi_{1} Y_{5}^{\prime}-\psi_{1}^{\prime} Y_{5}\right) Y_{1}^{\prime}-\left(\psi_{1} Y_{6}^{\prime}-\psi_{1}^{\prime} Y_{6}\right) Y_{1}\right) e^{\Delta_{7}}\right)^{-1} \\
\times & \frac{1}{2+i \omega / r} \frac{\nu_{0}}{D}\left(\left(\psi_{1} Y_{5}^{\prime}-\psi_{1}^{\prime} Y_{5}\right) Y_{3}^{\prime}-\left(\psi_{1} Y_{6}^{\prime}-\psi_{1}^{\prime} Y_{6}\right) Y_{3}\right) e^{\Delta_{7}} .
\end{aligned}
$$

Again, this form for linear response to a noise coded signal is the same for $r_{1}<0, r_{1}>0$ and $r<0$, although the derivation should be done separately for the three cases. The $r_{1}=0$ case can be obtained as a limit case from the above formula.

\subsection{3 $\quad r_{1}=0$ case}

The result for the $r_{1}=0$ could be obtained similar to that in Section 2.7. By doing the replacement

$$
\begin{aligned}
\psi_{5}(v) & =e^{a v}, \quad \psi_{6}(v)=\frac{1}{2 a} e^{-a v}, \\
Y_{5}(v) & =\frac{1}{2 a}\left(e^{a\left(v_{1}-v\right)}-e^{a\left(v-v_{1}\right)}\right), \\
Y_{6}(v) & =\frac{1}{2}\left(e^{a\left(v_{1}-v\right)}+e^{a\left(v-v_{1}\right)}\right),
\end{aligned}
$$

where $a=\sqrt{\frac{\left(\mu-v_{0}\right)^{2}}{4 D^{2}}-\frac{i \omega}{D}}$, and other replacement as given in the Appendix, we can get the linear response for $r_{1}=0$ from Eq. (4.40) and (4.53),

$$
\begin{aligned}
\nu_{1 c}^{\text {Low }}(\omega)= & \left(\psi_{1}\left(v_{r}\right) e^{\Delta_{0}+i \omega \tau_{r}}+\left(\left(\psi_{1} Y_{5}^{\prime}-\psi_{1}^{\prime} Y_{5}\right) Y_{1}^{\prime}-\left(\psi_{1} Y_{6}^{\prime}-\psi_{1}^{\prime} Y_{6}\right) Y_{1}\right) e^{\Delta_{7}}\right)^{-1} \\
& \left(\frac{1}{1-i \omega}\left(\psi_{1} P_{01}-\sqrt{D} \Phi_{1} P_{01}^{\prime}\right)-\frac{\nu_{0}}{\sqrt{D}} \frac{i \omega}{1-i \omega} \Phi_{1}\left(v_{r}\right) e^{\Delta_{0}+i \omega \tau_{r}}\right. \\
& \left.+\frac{1}{1+i \omega / r}\left(\left(\psi_{1} Y_{5}^{\prime}-\psi_{1}^{\prime} Y_{5}\right) P_{02}\left(v_{1}\right)+\left(\frac{1}{a} K_{1} \psi_{1}^{\prime}+K_{2} \psi_{1}\right) P_{02}^{\prime}\left(v_{1}\right)\right) e^{\Delta_{6}}\right) \\
\nu_{1 c}^{\text {High }}(\omega)= & \left(\psi_{1}\left(v_{r}\right) e^{\Delta_{0}+i \omega \tau_{r}}+\left(\left(\psi_{1} Y_{5}^{\prime}-\psi_{1}^{\prime} Y_{5}\right) Y_{1}^{\prime}-\left(\psi_{1} Y_{6}^{\prime}-\psi_{1}^{\prime} Y_{6}\right) Y_{1}\right) e^{\Delta_{7}}\right)^{-1} \\
& \times \frac{1}{1+i \omega / r} \frac{\nu_{0}}{\sqrt{r D}}\left(\left(\psi_{1} Y_{5}^{\prime}-\psi_{1}^{\prime} Y_{5}\right) Y_{2}^{\prime}-\left(\psi_{1} Y_{6}^{\prime}-\psi_{1}^{\prime} Y_{6}\right) Y_{2}\right) e^{\Delta_{7}},
\end{aligned}
$$


and

$$
\begin{aligned}
\nu_{1 n}^{\text {Low }}(\omega)= & \left(\psi_{1}\left(v_{r}\right) e^{\Delta_{0}+i \omega \tau_{r}}+\left(\left(\psi_{1} Y_{5}^{\prime}-\psi_{1}^{\prime} Y_{5}\right) Y_{1}^{\prime}-\left(\psi_{1} Y_{6}^{\prime}-\psi_{1}^{\prime} Y_{6}\right) Y_{1}\right) e^{\Delta_{7}}\right)^{-1} \\
\times & \left(\frac{1}{2-i \omega}\left(-\frac{i \omega}{\sqrt{D}} \phi_{1} P_{01}+2(i \omega-1) \Upsilon_{1} P_{01}^{\prime}\right)\right. \\
& +\frac{\nu_{0}}{D} \frac{i \omega(i \omega-1)}{2-i \omega} \Upsilon_{1}\left(v_{r}\right) e^{\Delta_{0}+i \omega \tau_{r}} \\
& +\frac{1}{D} \frac{i \omega}{2+i \omega / r}\left(\frac{1}{a} K_{1} \psi_{1}^{\prime}+K_{2} \psi_{1}\right) P_{02}\left(v_{1}\right) e^{\Delta_{6}} \\
& \left.+\frac{2}{2+i \omega / r}\left(\frac{1}{a} K_{3} \psi_{1}^{\prime}+K_{4} \psi_{1}\right) P_{02}^{\prime}\left(v_{1}\right) e^{\Delta_{6}}\right) \\
\nu_{1 n}^{\text {High }}(\omega)= & \left(\psi_{1}\left(v_{r}\right) e^{\Delta_{0}+i \omega \tau_{r}}+\left(\left(\psi_{1} Y_{5}^{\prime}-\psi_{1}^{\prime} Y_{5}\right) Y_{1}^{\prime}-\left(\psi_{1} Y_{6}^{\prime}-\psi_{1}^{\prime} Y_{6}\right) Y_{1}\right) e^{\Delta_{7}}\right)^{-1} \\
\times & \frac{1}{2+i \omega / r} \frac{\nu_{0}}{D}\left(\left(\psi_{1} Y_{5}^{\prime}-\psi_{1}^{\prime} Y_{5}\right) Y_{3}^{\prime}-\left(\psi_{1} Y_{6}^{\prime}-\psi_{1}^{\prime} Y_{6}\right) Y_{3}\right) e^{\Delta_{7}},
\end{aligned}
$$

where $K_{1}$ etc. are defined as

$$
\begin{aligned}
& K_{1}=\frac{1}{2}\left(\frac{1}{a+\frac{\mu}{2 D}} e^{a\left(v_{1}-v_{0}\right)}+\frac{1}{a-\frac{\mu}{2 D}} e^{a\left(v_{0}-v_{1}\right)}\right), \\
& K_{2}=\frac{1}{2}\left(\frac{1}{a+\frac{\mu}{2 D}} e^{a\left(v_{1}-v_{0}\right)}-\frac{1}{a-\frac{\mu}{2 D}} e^{a\left(v_{0}-v_{1}\right)}\right), \\
& K_{3}=\frac{1}{2}\left(\frac{a-\frac{\mu}{2 D}}{a+\frac{\mu}{2 D}} e^{a\left(v_{1}-v_{0}\right)}-\frac{a+\frac{\mu}{2 D}}{a-\frac{\mu}{2 D}} e^{a\left(v_{0}-v_{1}\right)}\right), \\
& K_{4}=\frac{1}{2}\left(\frac{a-\frac{\mu}{2 D}}{a+\frac{\mu}{2 D}} e^{a\left(v_{1}-v_{0}\right)}+\frac{a+\frac{\mu}{2 D}}{a-\frac{\mu}{2 D}} e^{a\left(v_{0}-v_{1}\right)}\right) .
\end{aligned}
$$

Note that in the limit $r_{1} \rightarrow 0, \Delta_{6}$ is simplified to $\Delta_{6}=\frac{1}{2 D}\left(v_{0}-\mu\right)\left(v_{1}-v_{0}\right)$.

\subsection{Limit behaviors}

\subsubsection{Large $\omega$ limit}

When $r>0$, the large $\omega$ limit can be obtained silimar to that in the last two chapters. Applying the large $\omega$ expansion as given in the appendix of Chapter 2 , the 3-piece models have the same large $\omega$ limit as the LIF model, $\nu_{1 c}(\omega) \rightarrow$ $\frac{\nu_{0}}{\sqrt{D}} \frac{1}{\sqrt{\omega}} e^{i \pi / 4}$ and $\nu_{1 n}(\omega) \rightarrow \frac{\nu_{0}}{D}$.

\subsection{2 $v_{1} \rightarrow v_{0}$ limit: relation to the previous models}

When $v_{1} \rightarrow v_{0}$ and $r\left(v_{1}-v_{t 0}\right) \rightarrow-v_{0}$, that is, continuous at $v_{0}$, the dynamics becomes the same as that for the $r-\tau_{m}$ model in Chapter 1. It can be easily checked that the linear response for 3-piece model reduces to that for the $r-\tau_{m}$ 
model. Similar limits also include $r_{1} \rightarrow r, r_{1} \rightarrow-1$ and $r \rightarrow \pm \infty$ with $r_{1}>0$. In all these limits, the results obtained for 3-piece model reduce to the $r-\tau_{m}$ model. These limits can serve as a cross-checking for the calculation in this chapter.

When $v_{1} \rightarrow v_{0}$, but $\dot{v}$ has a step change $\Delta \dot{v}$, the linear responses will reduce to the results obtained in Chapter 3. First, there is a step change for the derivative of the probability density at $v_{0}$ from Eq. (4.12),

$$
P_{02}^{\prime}\left(v_{0}\right)-P_{01}^{\prime}\left(v_{0}\right)=\frac{\Delta \dot{v}}{D} P_{01}\left(v_{0}\right)
$$

For the parabolic cylinder functions, there are the following limit behaviors which can be deduced from the recurrence relation for them,

$$
\begin{array}{ll}
Y_{5}\left(v_{0}\right) \rightarrow 0, & Y_{5}^{\prime}\left(v_{0}\right) \rightarrow-1, \\
Y_{6}\left(v_{0}\right) \rightarrow-\frac{\Delta \dot{v}}{2 D}, & Y_{6}^{\prime}\left(v_{0}\right) \rightarrow 0,
\end{array}
$$

and

$$
\begin{aligned}
& \sqrt{\left|r_{1}\right|} Y_{51}\left(v_{0}\right) \rightarrow \sqrt{D}, \\
& \sqrt{\left|r_{1}\right|} Y_{51}^{\prime}\left(v_{0}\right) \rightarrow-\frac{v_{0}-\mu}{2 \sqrt{D}}, \\
& r_{1} Y_{52}\left(v_{0}\right) \rightarrow v_{0}-\mu, \\
& r_{1} Y_{52}^{\prime}\left(v_{0}\right) \rightarrow i \omega-\frac{\left(v_{0}-\mu\right)^{2}}{2 D}, \\
& \sqrt{\left|r_{1}\right|}\left(\psi_{1}\left(v_{0}\right) Y_{51}^{\prime}\left(v_{0}\right)-\psi_{1}^{\prime} Y_{51}\left(v_{0}\right)\right) \rightarrow i \omega \phi_{1}\left(v_{0}\right) .
\end{aligned}
$$

Note also that $\Delta_{6} \rightarrow 0$ and $\Delta_{7} \rightarrow \Delta_{1}$ where $\Delta_{1}$ was defined in Eq. (3.31) in Chapter 3.

Substituting these into Eq. (4.40) and Eq. (4.53), we get

$$
\begin{aligned}
\nu_{1 c}^{\text {Low }}(\omega) \rightarrow & \left(\psi_{1}\left(v_{r}\right) e^{\Delta_{0}}+\left(Y_{1} \psi_{1}^{\prime}-Y_{1}^{\prime} \psi_{1}+\frac{\Delta \dot{v}}{2 D} \psi_{1} Y_{1}\right) e^{\Delta_{1}}\right)^{-1} \\
& \times\left(\frac{i\left(\omega+\omega^{\prime}\right)}{(1-i \omega)\left(1+i \omega^{\prime}\right)}\left(\psi_{1} P_{01}-\sqrt{D} \Phi_{1} P_{01}^{\prime}\right)-\frac{i \omega}{1-i \omega} \frac{\nu_{0}}{\sqrt{D}} \Phi_{1}\left(v_{r}\right) e^{\Delta_{0}}\right. \\
& \left.-\frac{i \omega^{\prime}}{1+i \omega^{\prime}} \frac{\Delta \dot{v}}{\sqrt{D}} \Phi_{1} P_{01}\right) \\
\nu_{1 c}^{H i g h}(\omega) \rightarrow & \left(\psi_{1}\left(v_{r}\right) e^{\Delta_{0}}+\left(Y_{1} \psi_{1}^{\prime}-Y_{1}^{\prime} \psi_{1}+\frac{\Delta \dot{v}}{2 D} \psi_{1} Y_{1}\right) e^{\Delta_{1}}\right)^{-1} \\
& \times \frac{1}{1+i \omega^{\prime}} \frac{\nu_{0}}{\sqrt{r D}} e^{\Delta_{1}}\left(\psi_{1}^{\prime} Y_{2}-\psi_{1} Y_{2}^{\prime}+\frac{\Delta \dot{v}}{2 D} \psi_{1} Y_{2}\right),
\end{aligned}
$$


and

$$
\begin{aligned}
\nu_{1 n}^{\text {Low }}(\omega) \rightarrow & \left(\psi_{1}\left(v_{r}\right) e^{\Delta_{0}}+\left(Y_{1} \psi_{1}^{\prime}-Y_{1}^{\prime} \psi_{1}+\frac{\Delta \dot{v}}{2 D} \psi_{1} Y_{1}\right) e^{\Delta_{1}}\right)^{-1} \\
& \times\left(\frac{i\left(\omega+\omega^{\prime}\right)}{(2-i \omega)\left(2+i \omega^{\prime}\right)}\left(-\frac{i \omega}{\sqrt{D}} \Phi_{1} P_{01}+2(i \omega-1) \Upsilon P_{01}^{\prime}\right)\right. \\
& \left.+\frac{i \omega(i \omega-1)}{2-i \omega} \frac{\nu_{0}}{D} \Upsilon\left(v_{r}\right) e^{\Delta_{0}}+\frac{\Delta \dot{v}}{D} \frac{i \omega^{\prime}}{2+i \omega^{\prime}}(i \omega-1) \Upsilon_{1} P_{01}\right) \\
\nu_{1 n}^{H i g h}(\omega) \rightarrow & \left(\psi_{1}\left(v_{r}\right) e^{\Delta_{0}}+\left(Y_{1} \psi_{1}^{\prime}-Y_{1}^{\prime} \psi_{1}+\frac{\Delta \dot{v}}{2 D} \psi_{1} Y_{1}\right) e^{\Delta_{1}}\right)^{-1} \\
& \times \frac{1}{2+i \omega^{\prime}} \frac{\nu_{0}}{D} e^{\Delta_{1}}\left(Y_{3} \psi_{1}^{\prime}-Y_{3}^{\prime} \psi_{1}+\frac{\Delta \dot{v}}{2 D} \psi_{1} Y_{3}\right)
\end{aligned}
$$

which are exactly the results we obtained in Chapter 3.

\subsubsection{Large $v_{b}$ limit}

When $r<0$, there is no large $v_{b}$ limit, since the argument $\frac{r\left(v_{b}-v_{t 0}\right)+\mu}{\sqrt{|r| D}}$ is usually not large. When $r>0$, the $v_{b}$ dependence can be put explicitly if $v_{b}$ is large. Eq. (2.59) in Chapter 2 can be applied here immediately,

$$
\begin{aligned}
\left(Y_{1} \psi_{1}^{\prime}-Y_{1}^{\prime} \psi_{1}\right) e^{\Delta_{1}} & =-\sqrt{D / r}\left(\phi_{12} \psi_{1}^{\prime}-\phi_{12}^{\prime} \psi_{1}\right) e^{\Delta_{v b}} \\
\left(Y_{2} \psi_{1}^{\prime}-Y_{2}^{\prime} \psi_{1}\right) e^{\Delta_{7}} & =-\sqrt{D / r} i \omega / r\left(\phi_{12} \psi_{1}^{\prime}-\phi_{12}^{\prime} \psi_{1}\right) e^{\Delta_{v b}} \frac{1}{x} \\
\left(Y_{3} \psi_{1}^{\prime}-Y_{3}^{\prime} \psi_{1}\right) e^{\Delta_{7}} & =\sqrt{D / r} i \omega(1+i \omega / r)\left(\phi_{12} \psi_{1}^{\prime}-\phi_{12}^{\prime} \psi_{1}\right) e^{\Delta_{v b}} \frac{1}{x^{2}}
\end{aligned}
$$

where $x=\frac{r\left(v_{b}-v_{t 0}\right)+\mu}{\sqrt{r D}}, \phi_{12}(v)=U\left(-i \omega / r+\frac{1}{2}, \frac{r\left(v-v_{t 0}\right)+\mu}{\sqrt{r D}}\right)$ and

$$
\begin{aligned}
\Delta_{v b}= & \left(\left(r_{1} v_{0}^{2}-2\left(r_{1} v_{t 1}-\mu\right) v_{0}\right)-\left(r_{1} v_{1}^{2}-2\left(r_{1} v_{t 1}-\mu\right) v_{1}\right)\right. \\
& +\frac{1}{r}\left(r\left(v_{1}-v_{t 0}+\mu\right)^{2}\right) / 4 D-i \omega / r \ln x
\end{aligned}
$$

Note that $\phi_{12}(v)$ satisfies the same parabolic cylinder equation as $\psi_{3}(v)$ and $\psi_{4}(v)$.

For $\nu_{1}^{\text {Low }}(\omega)$, only the denominator depends on $v_{b}$. In the large $v_{b}$ limit, the dependence exists through a complex phase. $\nu_{1}^{H i g h}(\omega)$, on the contrary, show a much stronger dependence on $v_{b}$. Substituting Eq. (4.66) into the $\nu_{1}^{\text {High }}$ parts of 
the linear responses, we get

$$
\begin{aligned}
\nu_{1 c}^{H i g h}(\omega) \simeq & \frac{\nu_{0}}{\sqrt{r D}} \frac{i \omega / r}{1+i \omega / r} \times \\
& \frac{-\sqrt{D / r}\left(\left(\psi_{1} Y_{5}^{\prime}-\psi_{1}^{\prime} Y_{5}\right) Y_{31}^{\prime}-\left(\psi_{1} Y_{6}^{\prime}-\psi_{1}^{\prime} Y_{6}\right) Y_{31}\right) e^{\Delta_{v b}}}{\psi_{1}\left(v_{r}\right) e^{\Delta_{0}+i \omega \tau_{r}}-\sqrt{D / r}\left(\left(\psi_{1} Y_{5}^{\prime}-\psi_{1}^{\prime} Y_{5}\right) \phi_{12}^{\prime}-\left(\psi_{1} Y_{6}^{\prime}-\psi_{1}^{\prime} Y_{6}\right) \phi_{12}\right) e^{\Delta_{v b}}} \\
\nu_{1 n}^{\text {High }}(\omega) \simeq & \frac{\nu_{0}}{D} \frac{i \omega(1+i \omega / r)}{2+i \omega / r} \times \\
& \frac{\sqrt{D / r}\left(\left(\psi_{1} Y_{5}^{\prime}-\psi_{1}^{\prime} Y_{5}\right) \phi_{12}^{\prime}-\left(\psi_{1} Y_{6}^{\prime}-\psi_{1}^{\prime} Y_{6}\right) \phi_{12}\right) e^{\Delta_{v b}}}{\psi_{1}\left(v_{r}\right) e^{\Delta_{0}+i \omega \tau_{r}}-\sqrt{D / r}\left(\left(\psi_{1} Y_{5}^{\prime}-\psi_{1}^{\prime} Y_{5}\right) \phi_{12}^{\prime}-\left(\psi_{1} Y_{6}^{\prime}-\psi_{1}^{\prime} Y_{6}\right) \phi_{12}\right) e^{\Delta_{v b}}}
\end{aligned}
$$

When $\omega$ is not small $(\omega \geq 1)$, the contribution from $\psi_{1}\left(v_{r}\right)$ term is negligible compared to the other terms in the denominators of the above equations. The expressions for $\nu_{1}^{H i g h}(\omega)$ are further simplified,

$$
\begin{aligned}
\nu_{1 c}^{H i g h}(\omega) & \simeq \frac{\nu_{0}}{r\left(v_{b}-v_{t 0}\right)+\mu} \frac{i \omega}{r+i \omega}, \\
\nu_{1 n}^{H i g h}(\omega) & \simeq-\frac{\nu_{0}}{\left(r\left(v_{b}-v_{t 0}\right)+\mu\right)^{2}} \frac{i \omega(1+i \omega / r)}{2+i \omega / r},
\end{aligned}
$$

Following the same arguments in the previous Chapters, the $\nu_{1}^{\text {High }}(\omega)$ parts represent the artifacts from the absorbing boundary. While the $\nu_{1}^{\text {Low }}(\omega)$ parts represent the biophysical relevant linear response. In the following we will consider only the $\nu_{1}^{\text {Low }}(\omega)$ parts in studying the properties of the linear response.

\subsection{Influence of details of AP generation on the dynamical response}

We are studying one-variable neuron models with the dynamics described by a piecewise linear function $f(v)$, Eq. (4.2). This means that the dynamics possesses discontinuity in the derivative of the $f(v)$ or even in $f(v)$ itself. For the latter case, we have investigates its influence on the dynamical response in last chapter. Here we will investigate the influence of the discontinuity in the derivative of $f(v)$, i.e., the influence of sharp edge, on the dynamical response. Some parameters of the models and of the stimulus will be used throughout this section: $v_{r}=0$, $v_{b}=10, \tau_{r}=0$, and $\mu=0, \sigma=0.6$.

\subsubsection{Influence of sub-threshold dynamics}

Since in our models a leak current and a linear spike generating current are always included, there is at leat one sharp edge existed in the subthreshold region. But 


\subsection{Influence of details of AP generation on the dynamical response81}

A

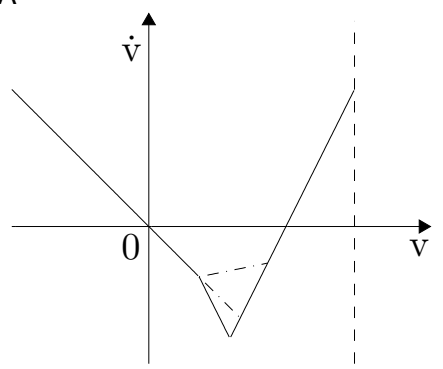

B

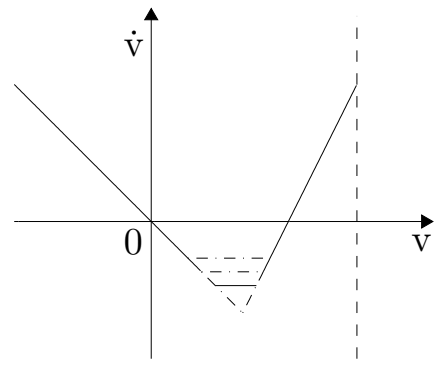

Figure 4.2: Schematic illustration of exaggerating the edge (A) and cutting the edge (B) within the subthreshold region.

most one-variable models, like the EIF and QIF models, have smooth subthreshold dynamics. To see how the sharp edge in the subthreshold dynamics influences the linear response, we start from the $r-\tau_{m}$ model and add the third piece to it by 'exaggerating the edge' or 'cutting the edge' within the subthreshold region, as illustrated in Fig. 4.2 $\mathrm{A}$ and B. The right piece of the $r-\tau_{m}$ model is described by $\dot{v}=1+\frac{1}{r}$ with $r=10$ and 100 . For exaggerating the edge, we fix $v_{0}=0.8$, and let the slope of the third piece change through $r_{1}=-2,-1,0.5$. For cutting the edge, we check three different choices of the rheobase current, $v_{0}=0.8,0.6,0.4$, and take $r_{1}=0$. These manipulations change the subthreshold dynamics and the rheobase currents. So we can expect the stationary firing rate will be changed, as shown in Fig. 4.3. Fig. 4.4 and 4.5 show the normalized linear response to both a current coded signal and a noise coded signal. We see the linear responses do not change much after these manipulations, although the subthreshold dynamics are obviously changed. However, the absolute value of the response amplitude does change due to the different rheobase current. This suggest that the dynamical responses are shaped mainly by the dynamics near the spike initiation threshold. Note that we are considering the noise driven regime. If there is a large mean input, then some manipulation stated above may result change for dynamics near the threshold and will be included in the situation in next section.

\subsubsection{Influence of spike initiation dynamics}

We consider three ways to modify the spike initiation dynamics in the 3-piece models, as illustrated in Fig. 4.6. In Fig. 4.6 A and B, the slopes of piece 1 and piece 2 are fixed, given by the lines $\dot{v}=-v$ and $\dot{v}=1+\frac{1}{r}$ respectively, while the slope of piece 3 is varied, within which the AP initiation threshold is passing. This can be done by either fixing the rheobase current $v_{0}$ and changing the slope of the third piece $r_{1}$ (Fig. 4.6 A), or by fixing the AP initiation threshold $v_{t 1}$ and changing the slope $r_{1}$ (Fig. 4.6B). In Fig. 4.6C , piece 1 and piece 3 are fixed and the slope $r$ of piece 2 is varied with the threshold crossing occurred within 

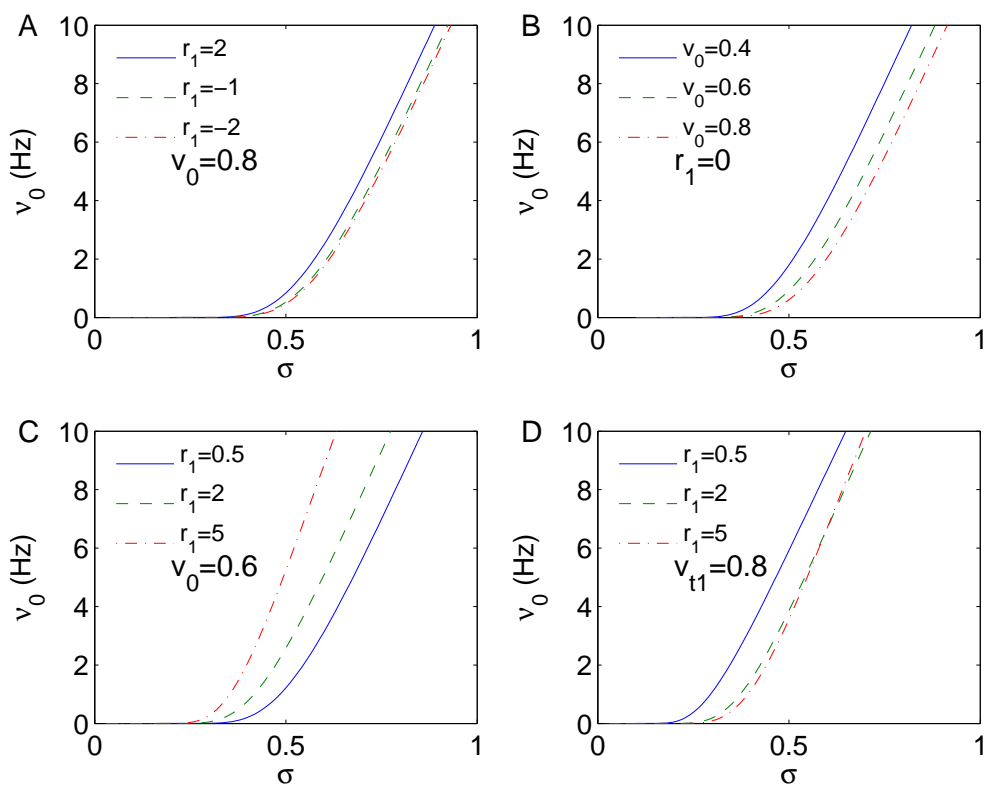

Figure 4.3: The stationary firing rate $\nu_{0}$ for the 3-piece model corresponding to the cases of exaggerating the edge $(\mathrm{A})$ and cutting the edge (B).
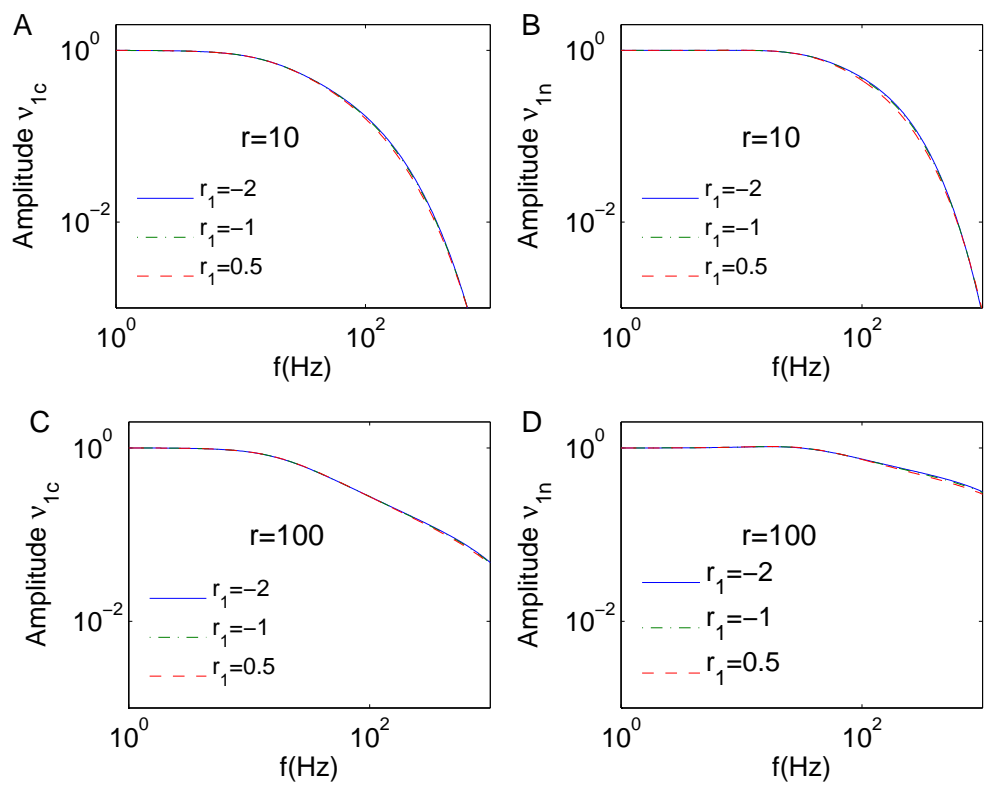

Figure 4.4: The normalized transmission functions $\left|\nu_{1}(\omega) / \nu_{1}(0.1)\right|$ for the 3-piece model from exacting the edge, corresponding to Fig. 4.2 A. Parameters: $v_{0}=0.8$, $r_{1}=-2,-1,0.5$. 


\subsection{Influence of details of AP generation on the dynamical response83}
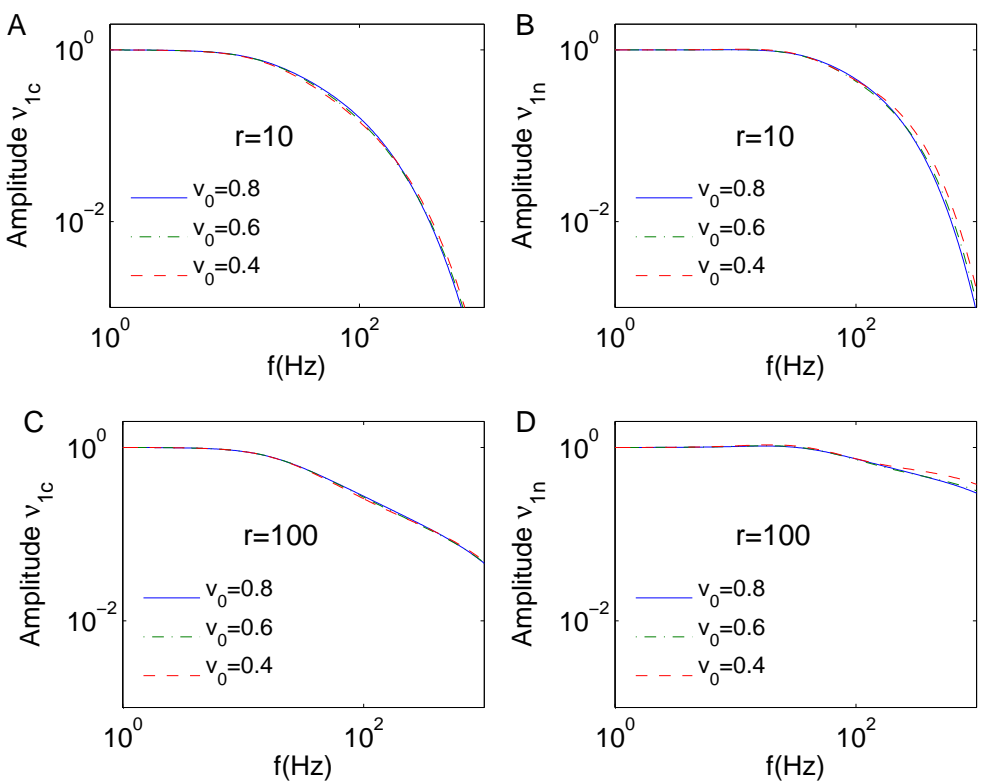

Figure 4.5: The normalized transmission functions $\left|\nu_{1}(\omega) / \nu_{1}(0.1)\right|$ for the 3-piece model from cutting the edge, corresponding to Fig. 4.2 B. Parameters: $v_{0}=$ $0.8,0.6,0.4, r_{1}=0$.

piece 3 or within piece 2. The linear responses are shown in Fig. 4.7, 4.8 and 4.9 for these cases. There is a transition between two different decaying behaviors, controlled by the two pieces in the spike generating current. We see that in the supra-threshold region, a sharp edge could cause local minimum developed in the transmission function for some specific signal frequency. This results from the interference of the two time constants in the spike initiation process.
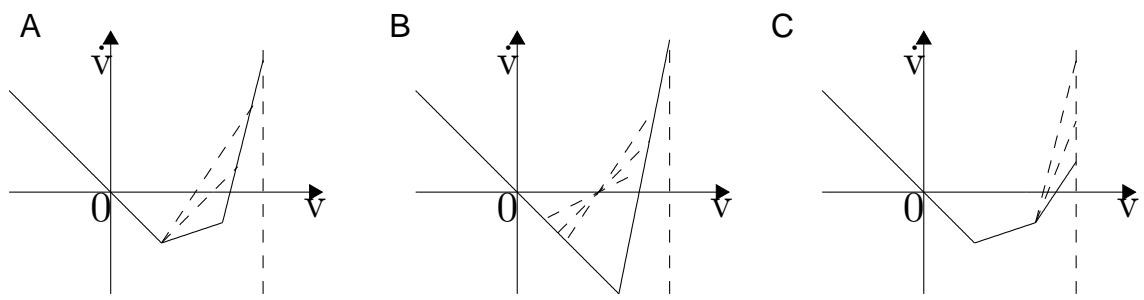

Figure 4.6: Schematic illustration of manipulations for the spike initiation dynamics. A, $v_{0}$ is fixed and $r_{1}$ is varied. $\mathrm{B}, \mathrm{AP}$ initiation threshold $v_{t 1}$, i.e., the zero crossing point of the piece 2 , is fixed and the slope $r_{1}$ is varied. C, The middle piece is fixed and the slope $r$ of piece 2 is varied. 

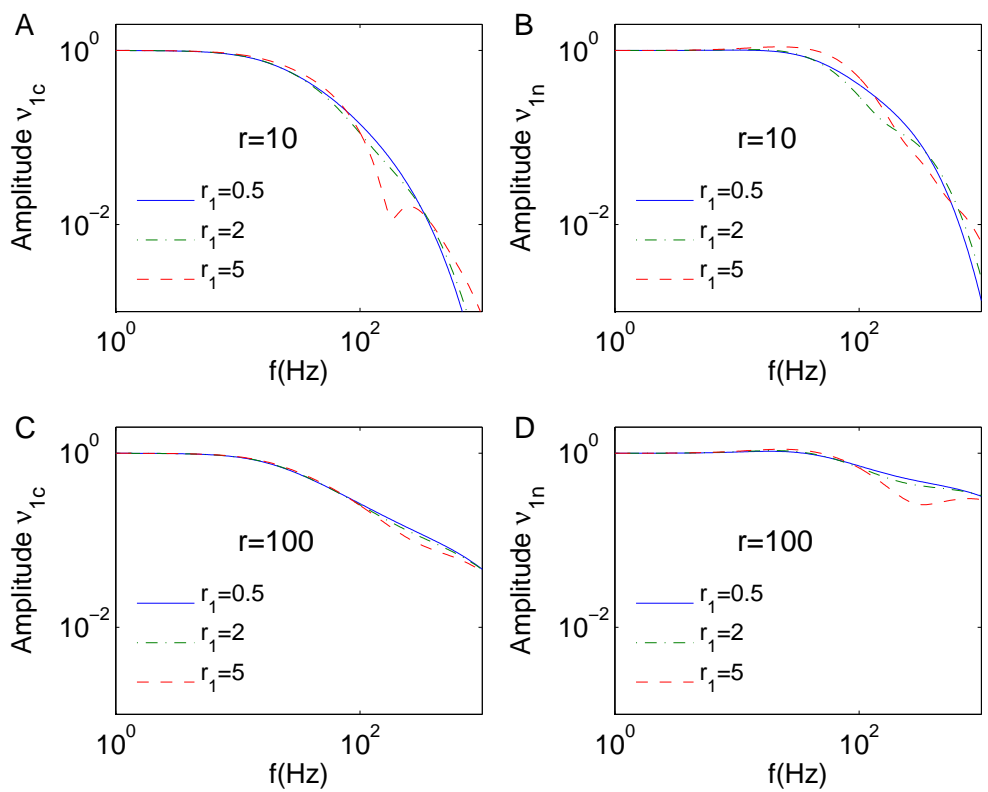

Figure 4.7: The normalized transmission functions $\left|\nu_{1}(\omega) / \nu_{1}(0.1)\right|$ for the 3-piece model corresponding to Fig. 4.6A. Parameters used are: $v_{0}=0.6, r_{1}=0.5,2,5$, $r=10,100$.
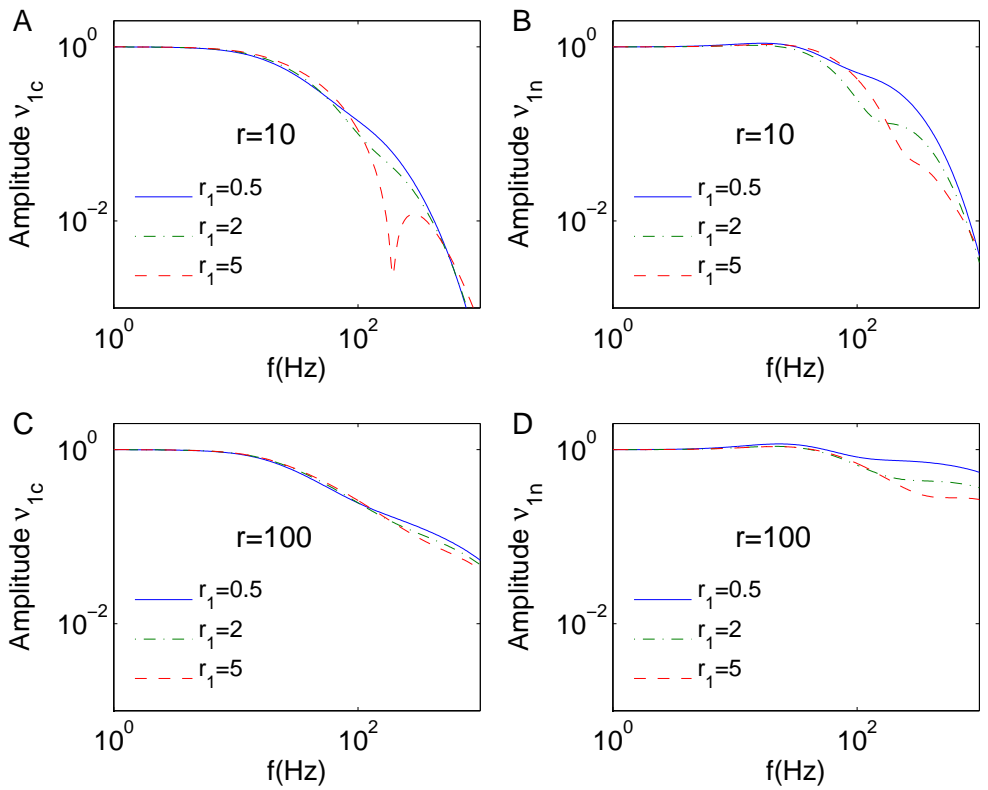

Figure 4.8: The normalized transmission functions $\nu_{1}(\omega) / \nu_{1}(0.1)$ for the 3-piece model corresponding to Fig. 4.6B. Parameters used are: $v_{t 1}=0.8, r_{1}=0.5,2,5$. 

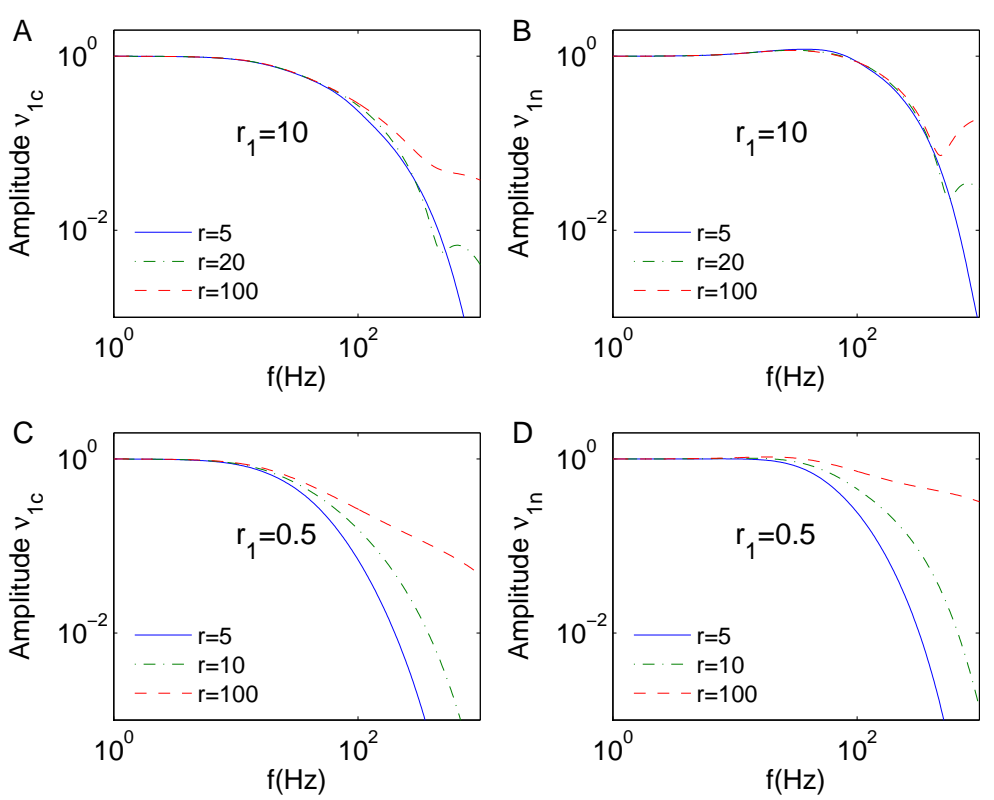

Figure 4.9: The normalized transmission functions $\nu_{1}(\omega) / \nu_{1}(0.1)$ for the 3-piece model corresponding to Fig. 4.6C . Parameters used are: $v_{0}=0.6, v_{1}=1$. For $\mathrm{A}$ and $\mathrm{B}, r_{1}=10, r=5,20,100$. Threshold crossing occurred within the middle piece (piece 3 ). For $\mathrm{C}$ and $\mathrm{D}, r_{1}=0.5, r=5,10,100$. Threshold crossing occurred within the right piece (piece 2). 


\subsection{Fitting the EIF model with a 3-piece model}

One question remained to be addressed is how the piecewise linear model is related to more biophysical realistic models. Here we will compare it with the EIF model, which can reproduce the conductance based models and the experimental data very well (Fourcaud-Trocme et al., 2003; Badel et al., 2008).

The EIF model is defined by the Langevin equation

$$
\tau_{m} \dot{V}=f(V)+\mu+\sigma \eta(t)
$$

with

$$
f(V)=V_{L}-V+\Delta T \exp \left(\frac{V-V_{T}}{\Delta T}\right),
$$

where $V$ is the membrane potential, $V_{L}$ is the resting potential, $V_{T}$ is the voltage threshold where $f^{\prime}\left(V_{T}\right)=0$. The membrane potential of the model neuron can reach infinity in a finite time, then it is reset to a resetting potential $V_{R}$ after a refractory period $\tau_{r}$.

Both the EIF and piecewise linear models include the AP initiation process explicitly characterized by passing an unstable fixed point of the membrane potential. There are three important characteristic quantities in the one-variable spiking neuron models: the rheobase current, the spike initiation threshold, which is the unstable fixed point, and the onset rapidness. We construct a 3-piece model as illustrated in Fig. 4.10. The left piece describes the leak current, the same as in the EIF model. The middle piece is taken to be tangent to the EIF model at $V_{T}$, which ensures the two models have the same rheobase current. The right piece passes the spike initiation threshold of the EIF model, which make the two models have the same unstable fixed points. The slope $r$ of the right piece is determined together with the absorbing boundary $v_{b}$ by fitting to the $f-I$ curve of the EIF model. The right piece crosses with the middle piece at $v_{1}$. The three piece model is then described by

$$
\tau_{m} \dot{v}=f(v)+\mu+\sigma \eta(t),
$$

where

$$
f(v)=\left\{\begin{array}{lrr}
-v, & -\infty<v \leq v_{0} & \text { piece } 1 \\
-v_{0}, & v_{0}<v \leq v_{1} & \text { piece } 3 \\
r\left(v-v_{t 0}\right), & v_{1}<v \leq v_{b} & \text { piece } 2
\end{array}\right.
$$

Here $v$ is the membrane potential relative to the resting potential. The $f-I$ curve of this model is given by

$$
\nu_{0}^{-1}=\log \frac{\mu-v_{r}}{\mu-v_{0}}+\frac{v_{1}-v_{0}}{\mu-v_{0}}+\frac{1}{r} \log \frac{r\left(v_{b}-v_{t 0}\right)+\mu}{\mu-v_{0}},
$$




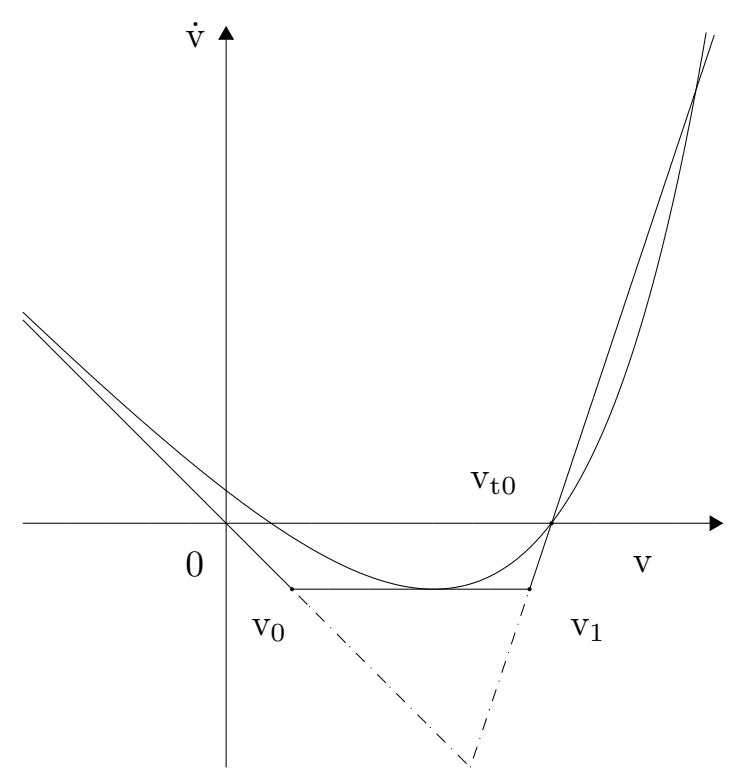

Figure 4.10: Illustration of fitting a 3-piece model to the EIF model. The left piece is from the leak current. The middle piece is tangent to the EIF model, so $v_{0}$ is fixed. The right piece passes the unstable fixed point $v_{t 0}$ with a slope chosen to fit the $f-I$ curve. Extending the left and right piece of the 3-piece model to cross at a point give a 2-piece model.

which describes a type I neuron, that is, the firing rate rising up from zero at the rheobase current. The first logistic term in the right side of above equation is characteristic for the LIF model, which is much faster and qualitative different from the square root behavior in the EIF model. But with the second term from the middle piece of the model which gives a linear rising f-I curve, the total rate of the initial rising is weakened and the f-I curves of the two models can match with each other. Note that the later growing of the $f-I$ curve is controlled mainly by the slope $r$ of the right piece.

In Badel et al. (2008) the dynamic $I-V$ curve of L5 pyramidal neurons are measured, which can be fitted by an EIF model very well. The parameters for the EIF model obtained there is: $V_{L}=-57 \mathrm{mV}, V_{T}=-42 \mathrm{mV}, \Delta T=1.51 \mathrm{mV}$, $\tau_{m}=17.2 \mathrm{~ms}$. Following the procedure outlined above we get $v_{0}=13.48$, $v_{t 0}=18.81$ in the corresponding 3-piece model. By fitting the $f-I$ curves for the two models, we find $r=100$ and $v_{b}=50$, as seen from Fig. 4.11A. With all the parameters fixed, we further check the $f-I$ curve with white noise. It turns out that the two models agree with each other quite well. Fig. 4.11B shows a comparison for $\sigma=10 \mathrm{mV}$.

From the 3-piece model, there is a corresponding 2-piece model (the $r-\tau_{m}$ model) obtained by extending the left and right piece in the 3-piece model with a crossing point at $v_{00}$. This 2-piece model has a larger rheobase current and 

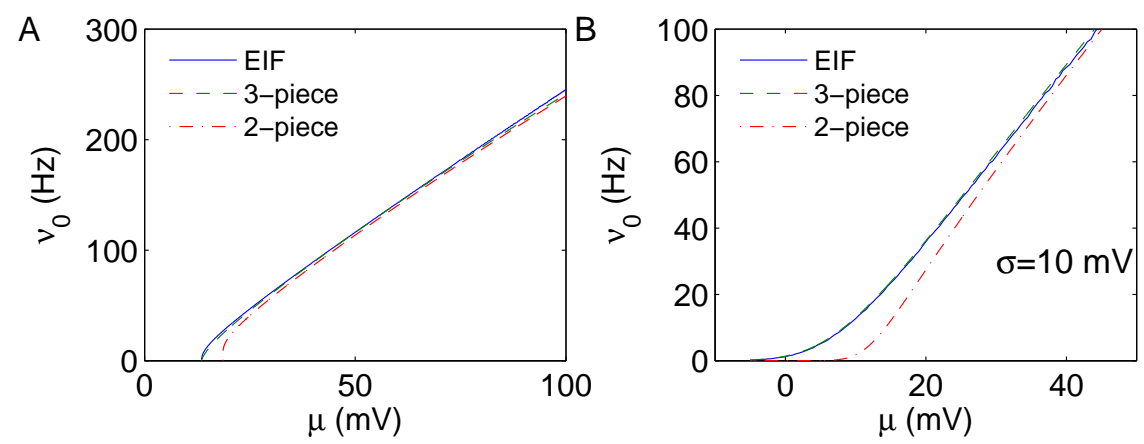

Figure 4.11: The $f-I$ curves for the EIF model and the 3-piece model. A, $f-I$ curves for a constant current; B, for a noisy current (Gaussian white noise, $\sigma=10 \mathrm{mV}$ ). The $f-I$ curve for the corresponding 2-piece model is also shown. The parameters for the EIF model are from experiments (Badel et al., 2008).

the $f-I$ curve does not agree with the EIF model well, especially for a noisy current (Fig. 4.11B). However, this 2-piece model is related to the 3-piece model by the cutting the edge procedure in the subthreshold region as we discussed in last section. We can therefore expect that they share the same linear response behavior. This reminds us that we can get other similar models by fixing $v_{0}$ and choosing a small slope $r_{1}$ for the middle piece. Then the $f-I$ curve of the EIF model can be fitted even better. But this change does not influence the value of $r$ and the linear response behavior.

Following the same procedure, we fit the EIF model with the parameters given in Fourcaud-Trocme et al. (2003), which are obtained from fitting the $f-$ I curve of the Wang-Buzsáki model (Wang \& Buzsáki, 1996) designed for the hippocampal interneurons. We reach the value $r \simeq 10$ for this EIF model. The difference between the onset rapidness reflects the different properties between cortical pyramidal neurons and hippocampal interneurons. Note that for the EIF model in Fourcaud-Trocme et al. (2003), different $v_{b}$ are required to fit the $f-I$ curve for constant and noisy currents with a range of input current as in Fig. 4.11 .

In Fig. 4.12 we compare the linear response of the EIF model and the 3-piece model with parameters determined above, which show a good agreement for low and intermediate signal frequencies. Note that the amplitude is not normalized. If we want to study the behavior of the linear response, like the cutoff frequency, then the 2-piece model also gives a good resemblance. This supports the usage of the 2-piece model in the previous chapters. 

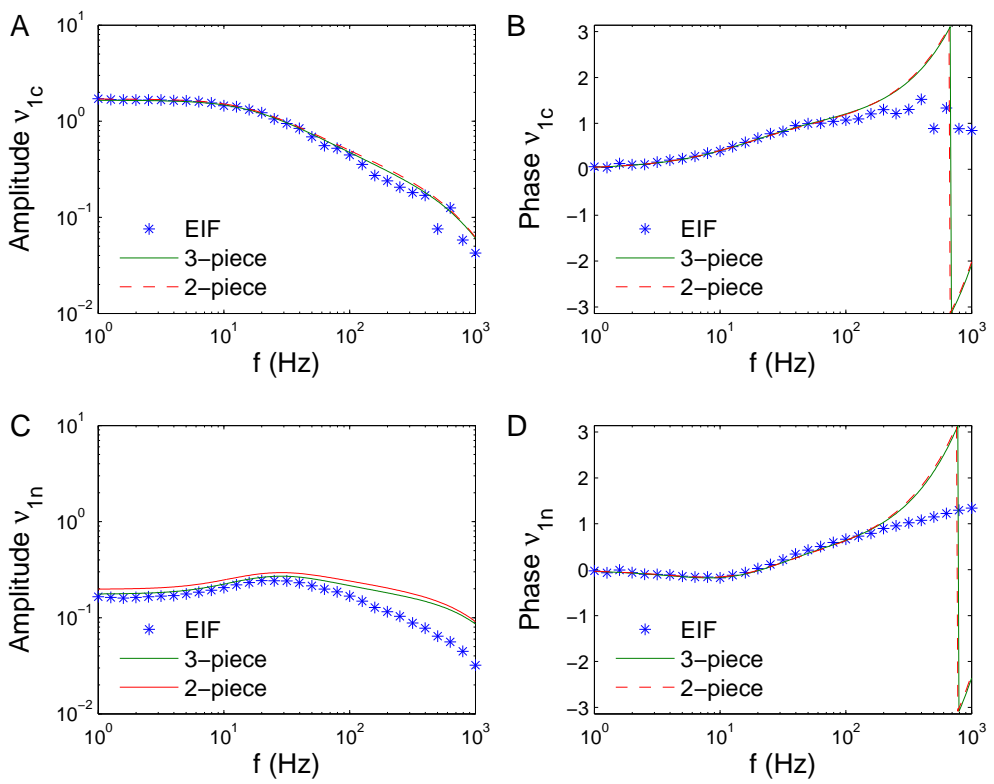

Figure 4.12: Comparison of the linear response for the EIF model (from Badel et al. (2008)) and the fitted 3-piece model. Parameter: $\mu=8.5 \mathrm{mV}, \sigma=10 \mathrm{mV}$, $\nu_{0}=10 \mathrm{~Hz}$.

\subsection{Signal encoding through barrier penetration in a bistable system}

For $r_{1}>0$ and $r<0$, the 3-piece model describes a bistable system, as illustrated in Fig. 4.1D. This corresponds to barrier penetration in a double well. The Kramers rate is the same as that for the two piece model in Chapter 2, since the curvature of the barrier is the same for the $r-\tau_{m}$ model and for the 3-piece model. The theoretical result for the stationary firing rate is given in the Appendix. To see the influence of $r_{1}$ and $r$, we choose their values as in Fig. 4.13 and plot the stationary firing rate $\nu_{0}$ for different $r_{1}$ and $r$. In Fig. 4.13A, we fixed the unstable fixed point and the slope $r$, while the slope $r_{1}$ takes values $1,10,100$. We see that the stationary firing rate decrease with the increasing of $r_{1}$ from Fig. 4.14 A and becomes insensitive to $r_{1}$ when $r_{1}$ is large. This results from the fact that the height of the barrier increases with $r_{1}$ when $r_{1}$ is small and insensitive to $r_{1}$ when $r_{1}$ is large. In Fig. 4.13 B, $r_{1}$ is fixed and $r$ change from 1, 10 and 100. We see that the stationary firing rate $\nu_{0}$ is insensitive to $r$.

The linear responses are decomposed into two pieces as for other cases of the piece-wise linear models. The $\nu_{1}^{H i g h}$ parts give the same high frequency behavior as in the LIF model, therefore reflect the contribution from the absorbing boundary. Specifically, the $\nu_{1 n}^{\text {High }}$ part gives an instantaneous component in the linear response. In Fig. 4.15 we show the dependence of the linear response on $r_{1}$ and 

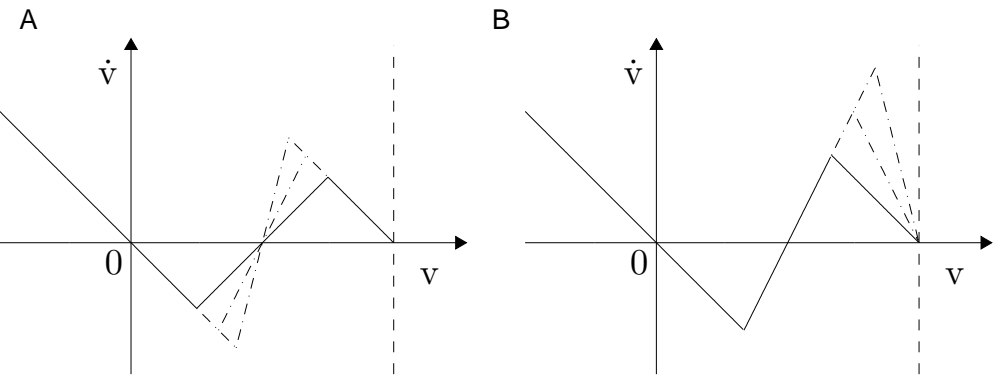

Figure 4.13: Schematic illustration of the 3-piece model describing a bistable system with different $r_{1}$ and $r$. A, $r$ is fixed and $r_{1}$ is variable; $\mathrm{B}, r_{1}$ is fixed and $r$ is variable.
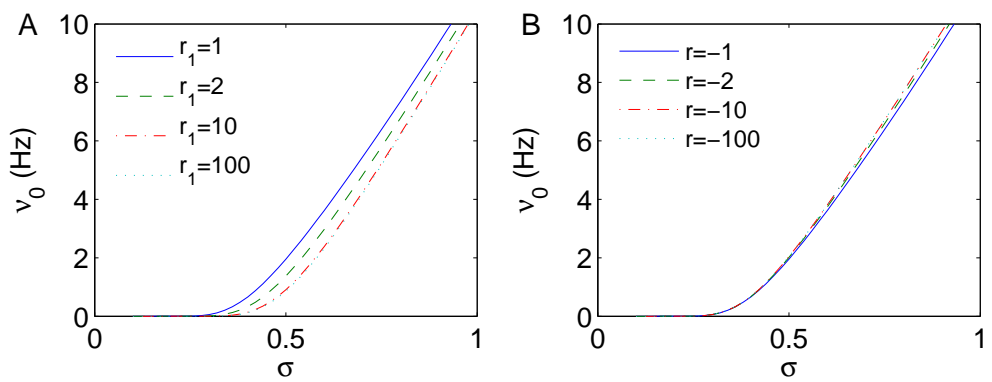

Figure 4.14: The stationary firing rate of the 3-piece model corresponding to Fig. 4.13 with $v_{t 1}=1$ and $v_{t 0}=2$. A, $r=-1$ and $r_{1}=1,10$ and $100 ; \mathrm{B}, r_{1}=10$ and $r=-1,-10$ and -100 . 

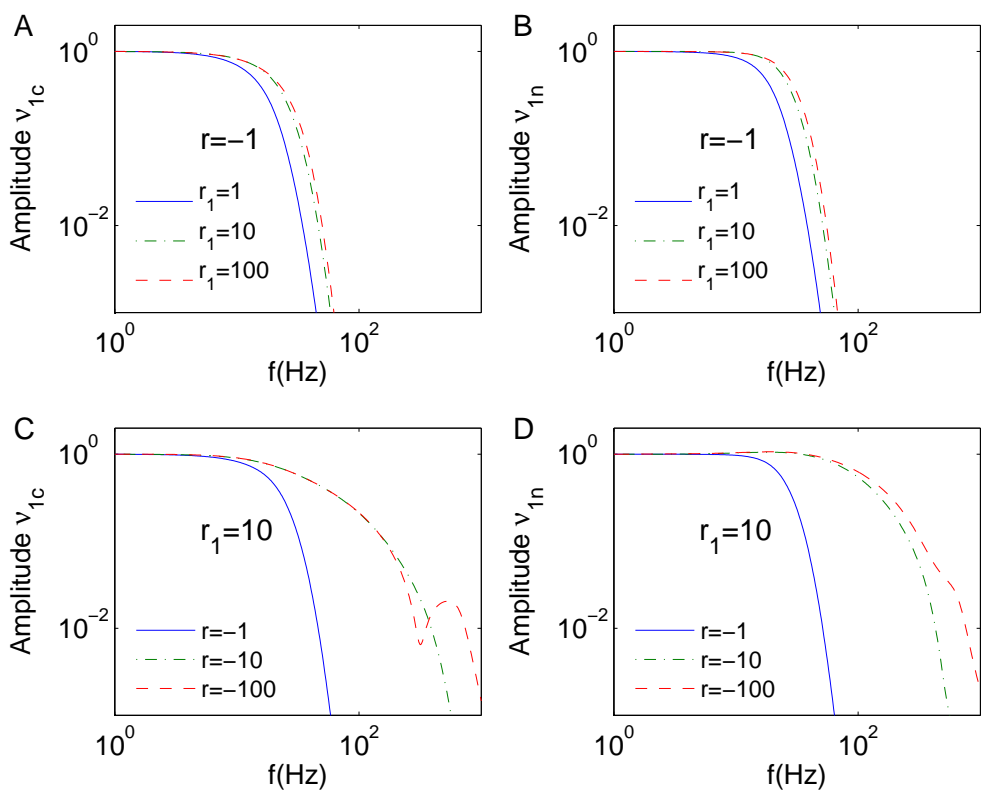

Figure 4.15: The linear response of the 3-piece model for a current coded signal (A and $\mathrm{C}$ ) and for a noise coded signal (B and D) corresponding to Fig. 4.13 with $v_{t 1}=1$ and $v_{t 0}=2$. A, B, $r=-1$ and $r_{1}=1,10$ and $100 ; \mathrm{C}, \mathrm{D}, r_{1}=10$ and $r=-1,-10$ and -100 .

$r$ corresponding to Fig. 4.13 with the right stable fixed point $v_{t 0}$ not far from the unstable fixed point. We see that the linear response does not change much with the increasing of $r_{1}$ when $r$ is fixed as shown in Fig. 4.15/A and B. The slope $r$ has a strong impact on the linear response and the cutoff frequencies are shifted significantly toward high signal frequency for a large $|r|$ as shown in Fig. 4.15C and D. There is also some interference occurred (Fig. 4.15 C), which produce some local minimum.

\subsection{Discussion: membrane dynamics and dynam- ical response}

Although the spike slope factor $\Delta T$ in the EIF model provides a measure for the speed of spike initiation, it is not very transparent to tell how fast the spike initiation dynamics is compared with the passive membrane dynamics. In other word, the spike slope factor $\Delta T$ controls the AP onset process but does not measure directly the onset rapidness. For instance, we do not know how much faster the spike initiation process in the cortical neurons than the hippocampal interneurons although both are well described by an EIF model with a slope factor $1.51 \mathrm{mV}$ and $3.48 \mathrm{mV}$ respectively. By fitting a 3-piece model to the EIF model, 
we approximate the exponential spike generating current with a linear current whose slope directly measures the ratio between the effective time constant for spike initiation and the membrane time constant. The piecewise linear model provides a good description for the $f-I$ curve with constant current, with noisy current and the linear response for low and intermediate signal frequencies. The resulting onset rapidness for the EIF model describing cortical pyramidal neurons and hippocampal interneurons are 100 and 10 respectively. We can see clearly that the AP initiation process is much faster for cortical neurons than for hippocampal interneurons. This large onset rapidness underlies the high cutoff frequency observed in cortical neurons. As we showed extensively in the last chapter, a large onset rapidness like 100 can easily push the cutoff frequencies to hundreds $\mathrm{Hz}$ when even a small correlation time is taken into account for a current coded signal. For a noise coded signal even white noise is enough to produce a hundreds $\mathrm{Hz}$ cutoff frequency for $r=100$, as showed in Chapter 2 . Note that the cutoff frequency is influenced also by the membrane time constant, therefore the difference between the cutoff frequencies for cortical neurons and interneurons might not be so large due to the smaller membrane time constant for interneurons.

The normalized behavior of the dynamical response, like the cutoff frequency, is found to be insensitive to the modification of subthreshold dynamics. The fitting results thus also support using the simpler 2-piece model as presented in Chapter 2 and Chapter 3. Following these steps from the EIF to 3-piece to 2-piece model, we reach a much simplified description for the spike initiation dynamics and at the same time keep qualitatively the essential dynamical response properties in the noise driven regime. In this sense, the piecewise linear model provides an analytically solvable and realistic model for the study of dynamical response of real neurons. 


\subsection{Appendix}

\section{Stationary solution simplified}

The stationary solution Eq. (4.14) can be further simplified. For $r_{1}>0$ and $r>0$,

$$
\begin{aligned}
P_{01}(v)= & \frac{\nu_{0}}{\sigma} e^{-\frac{1}{\sigma^{2}}(v-\mu)^{2}}\left(2 \int_{\left(\max \left(v, v_{r}\right)-\mu\right) / \sigma}^{\left(v_{0}-\mu\right) / \sigma} e^{y^{2}} d y\right. \\
& +\sqrt{\frac{\pi}{r_{1}}} e^{A}\left(\operatorname{Erfc}\left(\frac{-v_{0}+\mu}{\sqrt{r_{1}} \sigma}\right)-\operatorname{Erfc}\left(\frac{\dot{v}_{1}+\mu}{\sqrt{r_{1}} \sigma}\right)\right) \\
& \left.+\sqrt{\frac{\pi}{r}} e^{A+B}\left(\operatorname{Erfc}\left(\frac{\dot{v}_{1}+\mu}{\sqrt{r} \sigma}\right)-\operatorname{Erfc}\left(\frac{\dot{v}_{b}+\mu}{\sqrt{r} \sigma}\right)\right)\right) \\
P_{03}(v)= & \left.\frac{\nu_{0}}{\sigma} \sqrt{\pi} e^{\frac{1}{r_{1} \sigma^{2}}\left(r_{1}\left(v-v_{t 1}\right)+\mu\right)^{2}}\right) \\
& \left(\frac{1}{\sqrt{r_{1}}}\left(\operatorname{Erfc}\left(\frac{r_{1}\left(v-v_{t 1}\right)+\mu}{\sqrt{r_{1}} \sigma}\right)-\operatorname{Erfc}\left(\frac{\dot{v}_{1}+\mu}{\sqrt{r_{1}} \sigma}\right)\right)\right. \\
& \left.+\frac{1}{\sqrt{r}} e^{B}\left(\operatorname{Erfc}\left(\frac{\dot{v}_{1}+\mu}{\sqrt{r} \sigma}\right)-\operatorname{Erfc}\left(\frac{\dot{v}_{b}+\mu}{\sqrt{r} \sigma}\right)\right)\right) \\
P_{02}(v)= & \frac{\nu_{0}}{\sigma} \sqrt{\frac{\pi}{r}} e^{\frac{1}{r \sigma^{2}}\left(\mu+r\left(v-v_{t 0}\right)\right)^{2}}\left(\operatorname{Erfc}\left(\frac{r\left(v-v_{t 0}\right)+\mu}{\sqrt{r} \sigma}\right)-\operatorname{Erfc}\left(\frac{\dot{v}_{b}+\mu}{\sqrt{r} \sigma}\right)\right)
\end{aligned}
$$

where $\dot{v}_{1}=r\left(v_{1}-v_{t 0}\right)$ and $\dot{v}_{b}=r\left(v_{b}-v_{t 0}\right)$

$$
\begin{aligned}
A & =\frac{1}{\sigma^{2}}\left(1+\frac{1}{r_{1}}\right)\left(v_{0}-\mu\right)^{2} \\
B & =\frac{1}{\sigma^{2}}\left(\frac{1}{r}-\frac{1}{r_{1}}\right)\left(r\left(v_{1}-v_{t 0}\right)+\mu\right)^{2}
\end{aligned}
$$

The stationary firing rate $\nu_{0}$ can then be obtained by using the normalization 
condition of the probability density, $\int_{-\infty}^{v_{b}} P_{0}(v) d v=1$,

$$
\begin{aligned}
\nu_{0}^{-1}= & \sqrt{\pi} \int_{\left(\mu-v_{0}\right) / \sigma}^{\left(\mu-v_{r}\right) / \sigma} e^{y^{2}} \operatorname{Erfc}(y) d y \\
+ & \frac{\pi}{2} e^{A} \operatorname{Erfc}\left(\frac{\mu-v_{0}}{\sigma}\right) \times\left(\frac{1}{\sqrt{r_{1}}}\left(\operatorname{Erfc}\left(\frac{-v_{0}+\mu}{\sqrt{r_{1}} \sigma}\right)-\operatorname{Erfc}\left(\frac{\dot{v}_{1}+\mu}{\sqrt{r_{1}} \sigma}\right)\right)\right. \\
& \left.+\frac{1}{\sqrt{r}} e^{B}\left(\operatorname{Erfc}\left(\frac{\dot{v}_{1}+\mu}{\sqrt{r} \sigma}\right)-\operatorname{Erfc}\left(\frac{\dot{v}_{b}+\mu}{\sqrt{r} \sigma}\right)\right)\right) \\
+ & \sqrt{\pi} \int_{\left(-v_{0}+\mu\right) / \sqrt{r_{1}} \sigma}^{\left(\dot{v}_{1}+\mu\right) / \sqrt{r_{1}} \sigma} d y e^{y^{2}}\left(\frac{1}{r_{1}}\left(\operatorname{Erfc}(y)-\operatorname{Erfc}\left(\frac{\dot{v}_{1}+\mu}{\sqrt{r_{1}} \sigma}\right)\right)\right. \\
& \left.+\frac{1}{\sqrt{r r_{1}}} e^{B}\left(\operatorname{Erfc}\left(\frac{\dot{v}_{1}+\mu}{\sqrt{r} \sigma}\right)-\operatorname{Erfc}\left(\frac{\dot{v}_{b}+\mu}{\sqrt{r} \sigma}\right)\right)\right) \\
+ & \frac{\sqrt{\pi}}{r} \int_{\left(\dot{v}_{1}+\mu\right) / \sqrt{r} \sigma}^{\left(\dot{v}_{b}+\mu\right) / \sqrt{r} \sigma} d x e^{y^{2}}\left(\operatorname{Erfc}(y)-\operatorname{Erfc}\left(\frac{\dot{v}_{b}+\mu}{\sqrt{r} \sigma}\right)\right)+\tau_{r}
\end{aligned}
$$

For $r_{1}<0$ and $r>0$,

$$
\begin{aligned}
P_{01}(v)= & \frac{\nu_{0}}{\sigma} e^{-\frac{1}{\sigma^{2}}(v-\mu)^{2}}\left(2 \int_{\left(\max \left(v, v_{r}\right)-\mu\right) / \sigma}^{\left(v_{0}-\mu\right) / \sigma} e^{y^{2}} d y\right. \\
& +\frac{2}{\sqrt{-r_{1}}} e^{A} \int_{\frac{\dot{i}_{1}+\mu}{\sqrt{-r_{1} \sigma}}}^{\frac{-v_{0}+\mu}{\sqrt{-r_{1} \sigma}}} e^{y^{2}} d y \\
& \left.+\sqrt{\frac{\pi}{r}} e^{A+B}\left(\operatorname{Erfc}\left(\frac{\dot{v}_{1}+\mu}{\sqrt{r} \sigma}\right)-\operatorname{Erfc}\left(\frac{\dot{v}_{b}+\mu}{\sqrt{r} \sigma}\right)\right)\right), \\
P_{03}(v)= & \frac{\nu_{0}}{\sigma} e^{\frac{1}{r_{1} \sigma^{2}}\left(r_{1}\left(v-v_{t 1}\right)+\mu\right)^{2}}\left(\frac{2}{\sqrt{-r_{1}}} \int_{\frac{\dot{v}_{1}+\mu}{\sqrt{-r_{1} \sigma}}}^{\frac{r_{1}\left(v-v_{t 1}\right)+\mu}{\sqrt{-r_{1} \sigma}}} e^{y^{2}} d y\right. \\
& \left.+\sqrt{\frac{\pi}{r}} e^{B}\left(\operatorname{Erfc}\left(\frac{\dot{v}_{1}+\mu}{\sqrt{r} \sigma}\right)-\operatorname{Erfc}\left(\frac{\dot{v}_{b}+\mu}{\sqrt{r} \sigma}\right)\right)\right), \\
P_{02}(v)= & \frac{\nu_{0}}{\sigma} \sqrt{\frac{\pi}{r}} e^{\frac{1}{r \sigma^{2}}\left(r\left(v-v_{t 0}\right)+\mu\right)^{2}}\left(\operatorname{Erfc}\left(\frac{r\left(v-v_{t 0}\right)+\mu}{\sqrt{r} \sigma}\right)-\operatorname{Erfc}\left(\frac{\dot{v}_{b}+\mu}{\sqrt{r} \sigma}\right) 4.78\right)
\end{aligned}
$$


and

$$
\begin{aligned}
\nu_{0}^{-1}= & \sqrt{\pi} \int_{\left(\mu-v_{0}\right) / \sigma}^{\left(\mu-v_{r}\right) / \sigma} e^{y^{2}} \operatorname{Erfc}(y) d y \\
& +\frac{\sqrt{\pi}}{\sqrt{-r_{1}}} e^{A} \operatorname{Erfc}\left(\frac{\mu-v_{0}}{\sigma}\right) \int_{\frac{\dot{v}_{1}+\mu}{\sqrt{-r_{1}} \sigma}}^{\frac{-v_{0}+\mu}{\sqrt{r_{1}} \sigma}} e^{y^{2}} d y \\
& +\frac{\pi}{2 \sqrt{r}} e^{A+B} \operatorname{Erfc}\left(\frac{\mu-v_{0}}{\sigma}\right)\left(\operatorname{Erfc}\left(\frac{\dot{v}_{1}+\mu}{\sqrt{r} \sigma}\right)-\operatorname{Erfc}\left(\frac{\dot{v}_{b}+\mu}{\sqrt{r} \sigma}\right)\right) \\
& -\frac{\sqrt{\pi}}{r_{1}} \int_{\frac{\dot{y}_{1}+\mu}{\sqrt{-r_{1} \sigma}}}^{\frac{-v_{0}+\mu}{\sqrt{r_{1} \sigma}}} d y e^{y^{2}}\left(\operatorname{Erfc}(y)-\operatorname{Erfc}\left(\frac{-v_{0}+\mu}{\sqrt{-r_{1}} \sigma}\right)\right) \\
& +\frac{\pi}{2 \sqrt{-r r_{1}}} e^{B}\left(\operatorname{Erfc}\left(\frac{\dot{v}_{1}+\mu}{\sqrt{-r_{1}} \sigma}\right)-\operatorname{Erfc}\left(\frac{-v_{0}+\mu}{\sqrt{-r_{1}} \sigma}\right)\right) \\
& \times \frac{\sqrt{\pi}}{r} \int_{\left(\dot{v}_{1}+\mu\right) / \sqrt{r} \sigma}^{\left(\dot{v}_{b}+\mu\right) / \sqrt{r} \sigma} d x e^{y^{2}}\left(\operatorname{Erfc}(y)-\operatorname{Erfc}\left(\frac{\dot{v}_{b}+\mu}{\sqrt{r} \sigma}\right)\right)+\tau_{r} .
\end{aligned}
$$

For $r_{1}=0$ and $r>0$,

$$
\begin{aligned}
P_{01}(v)= & \frac{\nu_{0}}{\sigma} e^{-\frac{1}{\sigma^{2}}(v-\mu)^{2}}\left(2 \int_{\left(\max \left(v, v_{r}\right)-\mu\right) / \sigma}^{\left(v_{0}-\mu\right) / \sigma} e^{y^{2}} d y\right. \\
& +\frac{\sigma}{v_{0}-\mu} e^{\frac{1}{\sigma^{2}}\left(\mu^{2}-v_{0}^{2}\right)}\left(e^{2\left(v_{0}-\mu\right) v_{1} / \sigma^{2}}-e^{2\left(v_{0}-\mu\right) v_{0} / \sigma^{2}}\right) \\
& +\sqrt{\frac{\pi}{r}} e^{A_{1}}\left(\operatorname{Erfc}\left(\frac{\dot{v}_{1}+\mu}{\sqrt{r} \sigma}\right)-\operatorname{Erfc}\left(\frac{\dot{v}_{b}+\mu}{\sqrt{r} \sigma}\right)\right) \\
P_{03}(v)= & \frac{\nu_{0}}{\sigma}\left(\frac{\sigma}{v_{0}-\mu}\left(e^{2\left(v_{0}-\mu\right)\left(v_{1}-v\right) / \sigma^{2}}-1\right)\right. \\
& \left.+\sqrt{\frac{\pi}{r}} e^{\frac{1}{r \sigma^{2}}\left(r\left(v_{1}-v_{t 0}\right)+\mu\right)^{2}}\left(\operatorname{Erfc}\left(\frac{\dot{v}_{1}+\mu}{\sqrt{r} \sigma}\right)-\operatorname{Erfc}\left(\frac{\dot{v}_{b}+\mu}{\sqrt{r} \sigma}\right)\right)\right) \\
P_{02}(v)= & \frac{\nu_{0}}{\sigma} \sqrt{\frac{\pi}{r}} e^{\frac{1}{r \sigma^{2}}\left(r\left(v-v_{t 0}\right)+\mu\right)^{2}}\left(\operatorname{Erfc}\left(\frac{r\left(v-v_{t 0}\right)+\mu}{\sqrt{r} \sigma}\right)-\operatorname{Erfc}\left(\frac{\dot{v}_{b}+\mu}{\sqrt{r} \sigma}\right) 4.80\right)
\end{aligned}
$$


and

$$
\begin{aligned}
\nu_{0}^{-1}= & \sqrt{\pi} \int_{\left(\mu-v_{0}\right) / \sigma}^{\left(\mu-v_{r}\right) / \sigma} e^{y^{2}} \operatorname{Erfc}(y) d y \\
& +\frac{\sqrt{\pi}}{2} \frac{\sigma}{v_{0}-\mu} \operatorname{Erfc}\left(\frac{\mu-v_{0}}{\sigma}\right) e^{\frac{1}{\sigma^{2}}\left(\mu^{2}-v_{0}^{2}\right)}\left(e^{2\left(v_{0}-\mu\right) v_{1} / \sigma^{2}}-e^{2\left(v_{0}-\mu\right) v_{0} / \sigma^{2}}\right) \\
& +\frac{\pi}{2 \sqrt{r}} e^{A_{1}} \operatorname{Erfc}\left(\frac{\mu-v_{0}}{\sigma}\right)\left(\operatorname{Erfc}\left(\frac{\dot{v}_{1}+\mu}{\sqrt{r} \sigma}\right)-\operatorname{Erfc}\left(\frac{\dot{v}_{b}+\mu}{\sqrt{r} \sigma}\right)\right) \\
& +\frac{\sigma^{2}}{2\left(v_{0}-\mu\right)^{2}}\left(e^{2\left(v_{0}-\mu\right)\left(v_{1}-v_{0}\right) / \sigma^{2}}-1\right)-\frac{v_{1}-v_{0}}{v_{0}-\mu} \\
& +\frac{\sigma}{2\left(v_{0}-\mu\right)} \sqrt{\frac{\pi}{r}}\left(e^{2\left(v_{0}-\mu\right)\left(v_{1}-v_{0}\right) / \sigma^{2}}-1\right) e^{\frac{1}{r \sigma^{2}}\left(r\left(v_{1}-v_{t 0}\right)+\mu\right)^{2}} \\
& \times\left(\operatorname{Erfc}\left(\frac{\dot{v}_{1}+\mu}{\sqrt{r} \sigma}\right)-\operatorname{Erfc}\left(\frac{\dot{v}_{b}+\mu}{\sqrt{r} \sigma}\right)\right) \\
& +\frac{\sqrt{\pi}}{r} \int_{\left(\dot{v}_{1}+\mu\right) / \sqrt{r} \sigma}^{\left(\dot{v}_{b}+\mu\right) / \sqrt{r} \sigma} d x e^{y^{2}}\left(\operatorname{Erfc}(y)-\operatorname{Erfc}\left(\frac{\dot{v}_{b}+\mu}{\sqrt{r} \sigma}\right)\right)+\tau_{r}
\end{aligned}
$$

where $A_{1}=\frac{1}{\sigma^{2}}\left(\mu^{2}-v_{0}^{2}+2\left(v_{0}-\mu\right) v_{1}\right)+\frac{1}{r}\left(r\left(v_{1}-v_{t 0}\right)+\mu\right)^{2}$.

For $r_{1}>0$ and $r<0$,

$$
\begin{aligned}
P_{01}(v)= & \frac{\nu_{0}}{\sigma} e^{-\frac{1}{\sigma^{2}}(v-\mu)^{2}}\left(2 \int_{\left(\max \left(v, v_{r}\right)-\mu\right) / \sigma}^{\left(v_{0}-\mu\right) / \sigma} e^{y^{2}} d y\right. \\
& +\sqrt{\frac{\pi}{r_{1}}} e^{A}\left(\operatorname{Erfc}\left(\frac{-v_{0}+\mu}{\sqrt{r_{1}} \sigma}\right)-\operatorname{Erfc}\left(\frac{\dot{v}_{1}+\mu}{\sqrt{r_{1}} \sigma}\right)\right) \\
& \left.+\frac{2}{\sqrt{-r}} e^{A+B} \int_{\frac{\dot{i}_{b}+\mu}{\sqrt{-r} \sigma}}^{\frac{\dot{v}_{1}+\mu}{\sqrt{-r} \sigma}} e^{y^{2}} d y\right) \\
P_{03}(v)= & \frac{\nu_{0}}{\sigma} e^{\frac{1}{r_{1} \sigma^{2}}}\left(r_{1}\left(v-v_{t 1}\right)+\mu\right)^{2} \\
& \left(\sqrt{\frac{\pi}{r_{1}}}\left(\operatorname{Erfc}\left(\frac{r_{1}\left(v-v_{t 1}\right)+\mu}{\sqrt{r_{1}} \sigma}\right)-\operatorname{Erfc}\left(\frac{\dot{v}_{1}+\mu}{\sqrt{r_{1} \sigma}}\right)\right)\right. \\
& \left.+\frac{2}{\sqrt{-r}} e^{B} \int_{\frac{\dot{v}_{b}+\mu}{\sqrt{-r} \sigma}}^{\frac{\dot{v}_{1}+\mu}{\sqrt{-r} \sigma}} e^{y^{2}} d y\right) \\
P_{02}(v)= & \frac{2 \nu_{0}}{\sigma} \frac{1}{\sqrt{-r}} e^{\frac{1}{r \sigma^{2}}\left(r\left(v-v_{t 0}\right)+\mu\right)^{2}} \int_{\frac{\dot{x}_{b}+\mu}{\sqrt{-r} \sigma}}^{\frac{r\left(v-v_{t 0}\right)+\mu}{\sqrt{-r} \sigma}} e^{y^{2}} d y
\end{aligned}
$$


and

$$
\begin{aligned}
\nu_{0}^{-1}= & \sqrt{\pi} \int_{\left(\mu-v_{0}\right) / \sigma}^{\left(\mu-v_{r}\right) / \sigma} e^{y^{2}} \operatorname{Erfc}(y) d y \\
& +\sqrt{\pi} e^{A} \operatorname{Erfc}\left(\frac{\mu-v_{0}}{\sigma}\right) \times\left(\frac{\sqrt{\pi}}{2} \frac{1}{\sqrt{r_{1}}}\left(\operatorname{Erfc}\left(\frac{-v_{0}+\mu}{\sqrt{r_{1}} \sigma}\right)-\operatorname{Erfc}\left(\frac{\dot{v}_{1}+\mu}{\sqrt{r_{1}} \sigma}\right)\right)\right. \\
& \left.+\frac{1}{\sqrt{-r}} e^{B} \int_{\frac{\dot{b}_{b}+\mu}{\sqrt{-r} \sigma}}^{\frac{\dot{v}_{1}+\mu}{\sqrt{-r} \sigma}} e^{y^{2}} d y\right) \\
& +\int_{\left(-v_{0}+\mu\right) / \sqrt{r_{1}} \sigma}^{\left(\dot{v}_{1}+\mu\right) / \sqrt{r_{1}} \sigma} d y e^{y^{2}}\left(\frac{\sqrt{\pi}}{r_{1}}\left(\operatorname{Erfc}(y)-\operatorname{Erfc}\left(\frac{\dot{v}_{1}+\mu}{\sqrt{r_{1}} \sigma}\right)\right)\right. \\
& \left.+\frac{2}{\sqrt{-r r_{1}}} e^{B} \int_{\frac{\dot{v}_{b}+\mu}{\sqrt{-r} \sigma}}^{\frac{\dot{v}_{1}+\mu}{\sqrt{-r} \sigma}} e^{y^{2}} d y\right) \\
& -\frac{\sqrt{\pi}}{r} \int_{\frac{\dot{v}_{b}+\mu}{\sqrt{-r} \sigma}}^{\frac{\dot{i}_{1}+\mu}{\sqrt{-r} \sigma}} d y e^{y^{2}}\left(\operatorname{Erfc}(y)-\operatorname{Erfc}\left(\frac{\dot{v}_{1}+\mu}{\sqrt{-r} \sigma}\right)\right)+\tau_{r}
\end{aligned}
$$

\section{Definition of parabolic cylinder functions}

$r_{1}>0$ and $r>0$

$$
\begin{aligned}
\psi_{5}(v) & =U\left(-i \omega / r_{1}+\frac{1}{2},-\frac{v-v_{t 1}+\mu / r_{1}}{\sqrt{D / r_{1}}}\right)=D_{i \omega / r_{1}-1}\left(-\frac{v-v_{t 1}+\mu / r_{1}}{\sqrt{D / r_{1}}}\right) \\
\Phi_{5}(v) & =U\left(-i \omega / r_{1}-\frac{1}{2},-\frac{v-v_{t 1}+\mu / r_{1}}{\sqrt{D / r_{1}}}\right)=D_{i \omega / r_{1}}\left(-\frac{v-v_{t 1}+\mu / r_{1}}{\sqrt{D / r_{1}}}\right) \\
\Upsilon_{5}(v) & =U\left(-i \omega / r_{1}-\frac{3}{2},-\frac{v-v_{t 0}+\mu / r_{1}}{\sqrt{D / r_{1}}}\right)=D_{i \omega / r_{1}+1}\left(-\frac{v-v_{t 1}+\mu / r_{1}}{\sqrt{D / r_{1}}}\right) \\
\psi_{6}(v) & =\sqrt{\frac{\pi D}{2 r_{1}}} V\left(-i \omega / r_{1}+\frac{1}{2},-\frac{v-v_{t 1}+\mu / r_{1}}{\sqrt{D / r_{1}}}\right) \\
\Phi_{6}(v) & =\sqrt{\frac{\pi D}{2 r_{1}}} V\left(-i \omega / r_{1}-\frac{1}{2},-\frac{v-v_{t 1}+\mu / r_{1}}{\sqrt{D / r_{1}}}\right) \\
\Upsilon_{6}(v) & =\sqrt{\frac{\pi D}{2 r_{1}}} V\left(-i \omega / r_{1}-\frac{3}{2},-\frac{v-v_{t 1}+\mu / r_{1}}{\sqrt{D / r_{1}}}\right) \\
Y_{5}(v) & =\psi_{5}\left(v_{1}\right) \psi_{6}(v)-\psi_{6}\left(v_{1}\right) \psi_{5}(v) \\
Y_{6}(v) & =\psi_{5}^{\prime}\left(v_{1}\right) \psi_{6}(v)-\psi_{6}^{\prime}\left(v_{1}\right) \psi_{5}(v) \\
Y_{51}(v) & =\psi_{6}(v) \Phi_{5}\left(v_{1}\right)-i \omega / r_{1} \psi_{5}(v) \Phi_{6}\left(v_{1}\right) \\
Y_{52}(v) & =\psi_{6}(v) \Upsilon_{5}\left(v_{1}\right)-i \omega / r_{1}\left(1+i \omega / r_{1}\right) \psi_{5}(v) \Upsilon_{6}\left(v_{1}\right)
\end{aligned}
$$




$$
\begin{aligned}
& \psi_{6}(v) \Phi_{5}(v)-i \omega / r_{1} \psi_{5}(v) \Phi_{6}(v)=\sqrt{D / r_{1}} \\
& \psi_{6}^{\prime}(v) \Phi_{5}(v)-i \omega / r_{1} \psi_{5}(v)^{\prime} \Phi_{6}(v)=\frac{r_{1}\left(v-v_{t 1}+\mu\right)}{2 D} \sqrt{D / r_{1}} \\
& \psi_{6}(v) \Upsilon_{5}(v)-i \omega / r_{1}\left(1+i \omega / r_{1}\right) \psi_{5}(v) \Upsilon_{6}(v)=-\left(v-v_{t 1}+\mu / r_{1}\right) \\
& \psi_{6}^{\prime}(v) \Upsilon_{5}(v)-i \omega / r_{1}\left(1+i \omega / r_{1}\right) \psi_{5}^{\prime}(v) \Upsilon_{6}(v)=i \omega / r_{1}-\frac{\left(v-v_{t 1}+\mu / r_{1}\right)^{2}}{2 D / r_{1}}
\end{aligned}
$$

$Y_{5}$ and $Y_{6}$ satisfy the following relations:

$$
\begin{aligned}
& Y_{5}\left(v_{1}\right)=0, \quad Y_{6}\left(v_{1}\right)=1 \\
& Y_{5}^{\prime}\left(v_{1}\right)=-1, \quad Y_{6}^{\prime}\left(v_{1}\right)=0 \\
& Y_{6}(v) \psi_{5}\left(v_{1}\right)-Y_{5}(v) \psi_{5}^{\prime}\left(v_{1}\right)=\psi_{5}(v) \\
& Y_{6}(v) \psi_{6}\left(v_{1}\right)-Y_{5}(v) \psi_{6}^{\prime}\left(v_{1}\right)=\psi_{6}(v) \\
& Y_{6}^{\prime}(v) \psi_{5}\left(v_{1}\right)-Y_{5}^{\prime}(v) \psi_{5}^{\prime}\left(v_{1}\right)=\psi_{5}^{\prime}(v) \\
& Y_{6}^{\prime}(v) \psi_{6}\left(v_{1}\right)-Y_{5}^{\prime}(v) \psi_{6}^{\prime}\left(v_{1}\right)=\psi_{6}^{\prime}(v)
\end{aligned}
$$

$r_{1}<0$ and $r>0$

$$
\begin{aligned}
\psi_{5}(v) & =U\left(i \omega / r_{1}-\frac{1}{2},-\frac{v-v_{t 1}+\mu / r_{1}}{\sqrt{D /\left|r_{1}\right|}}\right)=D_{i \omega /\left|r_{1}\right|}\left(-\frac{v-v_{t 1}+\mu / r_{1}}{\sqrt{D /\left|r_{1}\right|}}\right) \\
\Phi_{5}(v) & =U\left(i \omega / r_{1}+\frac{1}{2},-\frac{v-v_{t 1}+\mu / r_{1}}{\sqrt{D /\left|r_{1}\right|}}\right)=D_{i \omega /\left|r_{1}\right|-1}\left(-\frac{v-v_{t 1}+\mu / r_{1}}{\sqrt{D /\left|r_{1}\right|}}\right) \\
\Upsilon_{5}(v) & =U\left(i \omega / r_{1}+\frac{3}{2},-\frac{v-v_{t 1}+\mu / r_{1}}{\sqrt{D /\left|r_{1}\right|}}\right)=D_{i \omega /\left|r_{1}\right|-2}\left(-\frac{v-v_{t 1}+\mu / r_{1}}{\sqrt{D /\left|r_{1}\right|}}\right) \\
\psi_{6}(v) & =\sqrt{\frac{\pi D}{2\left|r_{1}\right|}} V\left(i \omega / r_{1}-\frac{1}{2},-\frac{v-v_{t 1}+\mu / r_{1}}{\sqrt{D /\left|r_{1}\right|}}\right) \\
\Phi_{6}(v) & =\sqrt{\frac{\pi D}{2\left|r_{1}\right|}} V\left(i \omega / r_{1}+\frac{1}{2},-\frac{v-v_{t 1}+\mu / r_{1}}{\sqrt{D /\left|r_{1}\right|}}\right) \\
\Upsilon_{6}(v) & =\sqrt{\frac{\pi D}{2\left|r_{1}\right|}} V\left(i \omega / r_{1}+\frac{3}{2},-\frac{v-v_{t 1}+\mu / r_{1}}{\sqrt{D /\left|r_{1}\right|}}\right) \\
Y_{5}(v) & =\psi_{5}\left(v_{1}\right) \psi_{6}(v)-\psi_{6}\left(v_{1}\right) \psi_{5}(v) \\
Y_{6}(v) & =\psi_{5}^{\prime}\left(v_{1}\right) \psi_{6}(v)-\psi_{6}^{\prime}\left(v_{1}\right) \psi_{5}(v) \\
Y_{51}(v) & =\psi_{5}(v) \Phi_{6}\left(v_{1}\right)+i \omega / r_{1} \psi_{6}(v) \Phi_{5}\left(v_{1}\right) \\
Y_{52}(v) & =\psi_{5}(v) \Upsilon_{6}\left(v_{1}\right)-i \omega / r_{1}\left(1+i \omega / r_{1}\right) \psi_{6}(v) \Upsilon_{5}\left(v_{1}\right)
\end{aligned}
$$




$$
\begin{aligned}
& \psi_{5}(v) \Phi_{6}(v)+i \omega / r_{1} \psi_{6}(v) \Phi_{5}(v)=\sqrt{D /\left|r_{1}\right|} \\
& \psi_{5}^{\prime}(v) \Phi_{6}(v)+i \omega / r_{1} \psi_{6}(v)^{\prime} \Phi_{5}(v)=-\frac{v-v_{t 1}+\mu / r_{1}}{2 \sqrt{D /\left|r_{1}\right|}} \\
& \psi_{5}(v) \Upsilon_{6}(v)-i \omega / r_{1}\left(1+i \omega / r_{1}\right) \psi_{6}(v) \Upsilon_{5}(v)=-\left(v-v_{t 1}+\mu / r_{1}\right) \\
& \psi_{5}^{\prime}(v) \Upsilon_{6}(v)-i \omega / r_{1}\left(1+i \omega / r_{1}\right) \psi_{6}^{\prime}(v) \Upsilon_{5}(v)=i \omega / r_{1}+\frac{\left(v-v_{t 1}+\mu / r_{1}\right)^{2}}{2 D /\left|r_{1}\right|}
\end{aligned}
$$

$Y_{5}$ and $Y_{6}$ satisfy the following relations:

$$
\begin{aligned}
& Y_{5}\left(v_{1}\right)=0 ;, \quad Y_{6}\left(v_{1}\right)=1 ;, \quad Y_{5}^{\prime}\left(v_{1}\right)=-1 ;, \quad Y_{6}^{\prime}\left(v_{1}\right)=0 ; \\
& Y_{6}(v) \psi_{5}\left(v_{1}\right)-Y_{5}(v) \psi_{5}^{\prime}\left(v_{1}\right)=\psi_{5}(v) \\
& Y_{6}(v) \psi_{6}\left(v_{1}\right)-Y_{5}(v) \psi_{6}^{\prime}\left(v_{1}\right)=\psi_{6}(v) \\
& Y_{6}^{\prime}(v) \psi_{5}\left(v_{1}\right)-Y_{5}^{\prime}(v) \psi_{5}^{\prime}\left(v_{1}\right)=\psi_{5}^{\prime}(v) \\
& Y_{6}^{\prime}(v) \psi_{6}\left(v_{1}\right)-Y_{5}^{\prime}(v) \psi_{6}^{\prime}\left(v_{1}\right)=\psi_{6}^{\prime}(v)
\end{aligned}
$$

\section{$r_{1}>0$ and $r<0$}

When $r>0$, the parabolic cylinder functions and their combinations need were defined in Chapter 2. When $r<0$, some modifications are required,

$$
\begin{aligned}
\psi_{3}(v) & =U\left(-i \omega /|r|-\frac{1}{2},-\frac{v-v_{t 0}+\mu / r}{\sqrt{D /|r|}}\right)=D_{i \omega /|r|}\left(-\frac{v-v_{t 0}+\mu / r}{\sqrt{D /|r|}}\right) \\
\Phi_{3}(v) & =U\left(-i \omega /|r|+\frac{1}{2},-\frac{v-v_{t 0}+\mu / r}{\sqrt{D /|r|}}\right)=D_{i \omega /|r|-1}\left(-\frac{v-v_{t 0}+\mu / r}{\sqrt{D /|r|}}\right) \\
\Upsilon_{3}(v) & =U\left(-i \omega /|r|+\frac{3}{2},-\frac{v-v_{t 0}+\mu / r}{\sqrt{D /|r|}}\right)=D_{i \omega /|r|-2}\left(-\frac{v-v_{t 0}+\mu / r}{\sqrt{D /|r|}}\right) \\
\psi_{4}(v) & =\sqrt{\frac{\pi D}{2|r|}} V\left(-i \omega /|r|-\frac{1}{2},-\frac{v-v_{t 0}+\mu / r}{\sqrt{D /|r|}}\right) \\
\Phi_{4}(v) & =\sqrt{\frac{\pi D}{2|r|}} V\left(-i \omega /|r|+\frac{1}{2},-\frac{v-v_{t 0}+\mu / r}{\sqrt{D /|r|}}\right) \\
\Upsilon_{4}(v) & =\sqrt{\frac{\pi D}{2|r|}} V\left(-i \omega / r+\frac{3}{2},-\frac{v-v_{t 0}+\mu / r}{\sqrt{D /|r|}}\right) \\
Y_{1}(v) & =\psi_{3}(v) \psi_{4}\left(v_{b}\right)-\psi_{4}(v) \psi_{3}\left(v_{b}\right) \\
Y_{2}(v) & =\psi_{3}(v) \Phi_{4}\left(v_{b}\right)+i \omega / r \psi_{4}(v) \Phi_{3}\left(v_{b}\right) \\
Y_{3}(v) & =\psi_{3}(v) \Upsilon_{4}\left(v_{b}\right)-i \omega / r(1+i \omega / r) \psi_{4}(v) \Upsilon_{3}\left(v_{b}\right)
\end{aligned}
$$


and some useful formulas from the recurrence relations,

$$
\begin{aligned}
& \psi_{3}(v) \Phi_{4}(v)+i \omega / r \psi_{4}(v) \Phi_{3}(v)=\sqrt{D /|r|} \\
& \psi_{3}^{\prime}(v) \Phi_{4}(v)+i \omega / r \psi_{4}^{\prime}(v) \Phi_{3}(v)=-\frac{v-v_{t 0}+\mu / r}{2 \sqrt{D /|r|}} \\
& \psi_{3}(v) \Upsilon_{4}(v)-i \omega / r(1+i \omega / r) \psi_{4}(v) \Upsilon_{3}(v)=-\left(v-v_{t 0}+\mu / r\right) \\
& \psi_{3}^{\prime}(v) \Upsilon_{4}(v)-i \omega / r(1+i \omega / r) \psi_{4}^{\prime}(v) \Upsilon_{3}(v)=i \omega / r+\frac{\left(v-v_{t 0}+\mu / r\right)^{2}}{2 D /|r|}
\end{aligned}
$$

$r_{1}=0$ and $r>0$

In this case, the parabolic cylinder functions for piece 3 should be replaced by the following functions, $\psi_{5}(v)=e^{a v}, \psi_{6}(v)=\frac{1}{2 a} e^{-a v}$ where $a=\sqrt{\frac{\left(\mu-v_{0}\right)^{2}}{4 D^{2}}-\frac{i \omega}{D}}$. They satisfy the condition $\psi_{3}^{\prime}(v) \psi_{4}(v)-\psi_{3}(v) \psi_{4}^{\prime}(v)=1$. Correspondingly, according to the recurrence relations satisfied by the parabolic cylinder functions, Eq. (2.89), we define

$$
\begin{aligned}
& \phi_{5}(v)=\sqrt{\frac{D}{r}}\left(\psi_{5}^{\prime}(v)-\frac{\mu-v_{0}}{2 D} \psi_{5}(v)\right)=\sqrt{\frac{D}{r}}\left(a-\frac{\mu-v_{0}}{2 D}\right) e^{a v} \\
& \Upsilon_{5}(v)=\sqrt{\frac{D}{r}}\left(\Phi_{5}^{\prime}(v)-\frac{\mu-v_{0}}{2 D} \Phi_{5}(v)\right)=\frac{D}{r}\left(a-\frac{\mu-v_{0}}{2 D}\right)^{2} e^{a v} \\
& \phi_{6}(v)=\frac{\sqrt{r D}}{i \omega}\left(\psi_{6}^{\prime}(v)-\frac{\mu-v_{0}}{2 D} \psi_{6}(v)\right)=-\frac{\sqrt{r D}}{i \omega}\left(a+\frac{\mu-v_{0}}{2 D}\right) \frac{1}{2 a} e^{-a v} \\
& \Upsilon_{6}(v)=\frac{\sqrt{r D}}{i \omega}\left(\Phi_{6}^{\prime}(v)-\frac{\mu-v_{0}}{2 D} \Phi_{6}(v)\right)=\frac{r D}{(i \omega)^{2}}\left(a+\frac{\mu-v_{0}}{2 D}\right)^{2} \frac{1}{2 a} e^{-a v}
\end{aligned}
$$

and

$$
\begin{aligned}
Y_{5}(v) & =\psi_{5}\left(v_{1}\right) \psi_{6}(v)-\psi_{6}\left(v_{1}\right) \psi_{5}(v) \\
& =\frac{1}{2 a}\left(e^{a\left(v_{1}-v\right)}-e^{a\left(v-v_{1}\right)}\right) \\
Y_{6}(v) & =\psi_{5}^{\prime}\left(v_{1}\right) \psi_{6}(v)-\psi_{6}^{\prime}\left(v_{1}\right) \psi_{5}(v) \\
& =\frac{1}{2 a}\left(e^{a\left(v_{1}-v\right)}+e^{a\left(v-v_{1}\right)}\right) \\
Y_{51}(v) & =\psi_{6}(v) \Phi_{5}\left(v_{b}\right)-\frac{i \omega}{r_{1}} \psi_{5}(v) \Phi_{6}\left(v_{1}\right) \\
& =\frac{1}{2 a} \sqrt{\frac{D}{r_{1}}}\left(\left(a-\frac{\mu-v_{0}}{2 D}\right) e^{a\left(v_{1}-v\right)}+\left(a+\frac{\mu-v_{0}}{2 D}\right) e^{a\left(v-v_{1}\right)}\right) \\
Y_{52}(v) & =\psi_{6}(v) \Upsilon_{5}\left(v_{1}\right)-\frac{i \omega}{r_{1}}\left(1+\frac{i \omega}{r_{1}}\right) \psi_{5}(v) \Upsilon_{6}\left(v_{1}\right) \\
& =\frac{1}{2 a} \frac{D}{r_{1}}\left(\left(a-\frac{\mu-v_{0}}{2 D}\right)^{2} e^{a\left(v_{1}-v\right)}-\left(a+\frac{\mu-v_{0}}{2 D}\right)^{2} e^{a\left(v-v_{1}\right)}\right)
\end{aligned}
$$




\section{Chapter 5}

\section{Dynamical AP encoding in spatially extended neurons}

In preceding chapters we treated a neuron as a "point" constructing one-variable spiking neuron models to study the AP encoding properties for time-varying signals. Real neurons, however, are spatially extended objects with many functional parts, like the soma, axon, dendrite and Ranvier nodes. Where the APs are generated could play an important role in the response properties of cortical neurons.

Axonal AP initiation has long been observed in cortical neurons (for a review, see Stuart et al. (1997)). The exact location, however, has only recently been found in the proximal part of the axon, the axon initial segment(AIS), using voltage sensitive dye imaging (Palmer \& Stuart, 2006). Further experiments have confirmed this observation (Inda et al., 2006; Hedstrom \& Rasband, 2006; Meeks \& Mennerick, 2007; Yu et al., 2008; Kole et al., 2008; Fleidervish et al., 2010). Patch-clamp and imaging experiments have revealed that the physiological properties of the AIS are different from the soma (Clark et al., 2009). The AIS has a higher sodium channel density than the soma, but there is a discrepancy between the ratios obtained from different approaches, ranging from larger than 10 fold to about 3 fold (Kole et al., 2008; Fleidervish et al., 2010). The threshold for AP generation is lower at the AIS than at the soma (Kole \& Stuart, 2008). The passive membrane time constant at the AIS might also be different for the soma, AIS and axon. For the GABAergic pyramidal cells there are axo-axonic synapses specially targeted at the AIS from Chandelier cells (for a review, see e.g., Howard et al. (2005)), which can modify the input conductance there and therefore, the passive membrane time constant and the electrotonic length at the AIS.

The functional implication of the AP initiation at the AIS is not well understood. APs back propagate to the soma after their generation at the AIS, which was reported to make the AP waveform at the soma develop a faster initiation speed and was suggested to explain the fast AP onset observed in cortical neurons 
(McCormick et al., 2007; Yu et al., 2008). But the effectiveness of this mechanism is still in controversy (Naundorf et al., 2007; Baranauskas et al., 2010). To realize it in multi-compartment modeling, a very high sodium channel density at the AIS and a long passive dendrite are required, which seems not to be fulfilled in real neurons (Huang, 2009; Baranauskas et al., 2010). Recently it was suggested that the latency resulting from AIS initiation of APs can enhance coincidence detection in the auditory system (Kuba et al., 2006). Furthermore, the position and length of the AIS were found to be plastic due to experience (Grubb \& Burrone, 2010; Kuba et al., 2010).

In this chapter we will study the functional effect of the axonal AP generation on signal encoding by combining the linear cable equation with the Gaussian neuron model, which assumes the membrane potential as a Gaussian random field and AP generation as a threshold crossing process. The axon will be taken as a homogeneous cable and the soma as a point at the end of the cable. This framework allows for an analytical investigation of the voltage coherence along the cable and the signal encoding properties. A boundary condition which incorporates the sub-threshold dynamics will be assigned at the soma. We will first derive the Green's functions for a homogeneous cable of one or two compartments. We then study the transmission of the correlation function along the axon and calculate the spike-triggered average voltage and spike-triggered variance at soma when spikes are generated at AIS. We further study the dynamical response at axon when a signal is injected into soma. The simplicity of the Gaussian neuron model allows theses important quantities to be studied analytically.

\subsection{The cable equation}

The cable theory provides an important theoretical framework to investigate the sub-threshold dynamics of spatial extended neurons (Tuckwell, 1988). Taking into account the 'core conductor' property, that the resistance for ions flowing across the membrane is much larger than flowing along the cable, the nerve cylinder can be described by the one dimensional cable equation. For a homogenous axon, the cable equation reads

$$
c_{m} \frac{\partial V(x, t)}{\partial t}=\frac{r_{a}}{2 r_{L}} \frac{\partial^{2} V(x, t)}{\partial x^{2}}-i_{m}
$$

where $V$ is the membrane potential relative to the resting potential, $c_{m}$ the specific membrane capacitance, $i_{m}$ the membrane current per unit area, $r_{a}$ the radius of axon and $r_{L}$ the intracellular resistivity. Taking the linear approximation for the membrane current, $i_{m}=V / r_{m}$ with $r_{m}$ the specific membrane resistance, the linear cable equation is given by

$$
\tau_{m} \frac{\partial V(x, t)}{\partial t}=\lambda^{2} \frac{\partial^{2} V(x, t)}{\partial x^{2}}-V(x, t)
$$


A

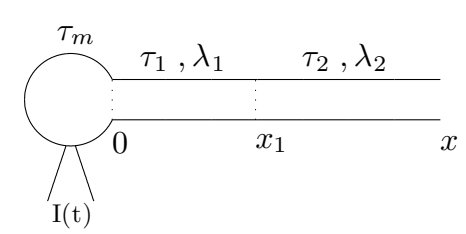

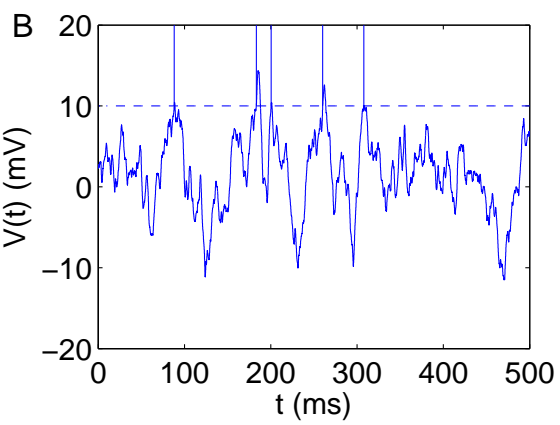

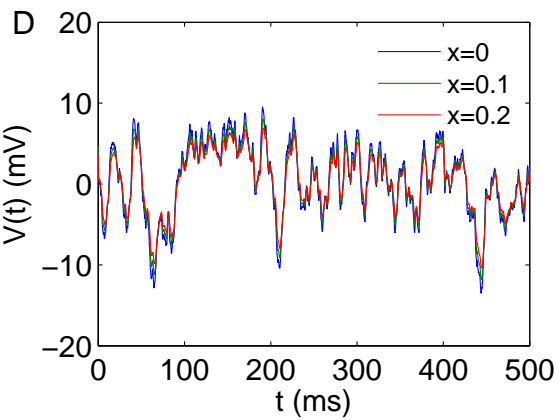

Figure 5.1: Illustration of the geometry and dynamics of the model used. A, the two-compartment model; B, the Gaussian neuron model; $\mathrm{C}$, the correlation function for the membrane potential at the soma as given by Eq. (5.38); D, the membrane potential at soma, the middle of the AIS and the end of the AIS.

where $\tau_{m}=c_{m} r_{m}$ is the membrane time constant and $\lambda=\sqrt{\frac{r_{a} r_{m}}{2 r_{L}}}$ the electrotonic length. $\lambda$ sets the spatial scale of the cable, which is about $400 \mu \mathrm{m}$ at the axon (Shu et al., 2006). In the linear cable equation (5.2), only the leak current is included. We will use a two-compartment homogenous cable to model the AIS initiation of APs in real neurons, with each compartment describes by Eq. (5.2), as illu in Fig. 5.1A. Details of the model will be given later. For numerical simulation of real neurons, multi-compartment models are used extensively, in which other ion currents, like the sodium current and potassium current for spike generating, will be included in the membrane current $i_{m}$ in Eq. (5.1).

The cable equation was found of importance in describing the neuron axon in 1940s (Hodgkin \& Rushton, 1946). The Rall model was then developed for dendritic neurons (Rall, 1959, 1977). Assuming a homogeneous distribution of synaptic inputs along the cable modeled as either a white noise or an OU process (colored noise), the coherence properties, like the variance and covariance of the membrane potential, were analyzed theoretically (Wan \& Tuckwell, 1979; Tuckwell \& Walsh, 1983; Tuckwell et al., 1984, 2002). Here we will combine the linear cable equation and the Gaussian neuron model to investigate the functional effect of AP initiation at the AIS. 


\subsection{Membrane potential as a Gaussian random field}

Gaussian random fields provide an important tool to study the threshold crossing process in neuroscience (Jung, 1994). In Wolf \& Geisel (1998) it was used to investigate the singular structure in the orientation map in visual cortex. The model is then used to study the functional implication of large threshold variability in AP generation in cortical neurons (Naundorf, 2005), and the correlation property of pair neurons (Burak et al., 2009; Tchumatchenko et al., 2010).

In the Gaussian neuron model, the stochastic property of the membrane potential is described by a stationary smooth Gaussian random field with a given correlation function, $C(\tau)=\langle V(t)(V(t+\tau))\rangle$. A spike is generated whenever the membrane potential cross a threshold from below (Fig. 5.1B). The formal results of the model are expressed with $C(\tau)$ and its derivatives. The advantage of this model is its analytical solvability for many important quantities, like the spike-triggered average and the dynamic response, which are accessible only numerically or analytically tractable only in very limited cases in the Fokker-Planck equation formalism, as used in preceding chapters.

In the rest of this section, we will outline the model for a point neuron following Naundorf (2005) . The spike activity is described by the neural response function $\rho(t)=\sum_{j} \delta\left(t-t^{j}\right)$ where $t^{j}$ is the time for the $j$ th spike and $\delta(t)$ is the Dirac $\delta$ function which satisfies $\int_{-\infty}^{+\infty} \delta(t) d t=1$. In the Gaussian neuron model,

$$
\rho(t)=\delta\left(V(t)-\psi_{0}\right)|\dot{V}| \Theta(\dot{V}(t))
$$

where $\psi_{0}$ is a constant threshold and $\Theta(x)$ is the Heaviside step function used in previous chapters. The step function in above expression ensures that only the threshold crossing from below is counted. The instantaneous firing rate is given by

$$
\nu(t)=\int_{-\infty}^{+\infty} d V(t) d \dot{V}(t) \delta\left(V(t)-\psi_{0}\right)|\dot{V}| \Theta(\dot{V}(t)) P(V(t), \dot{V}(t)),
$$

where $P(V(t), \dot{V}(t))$ is the joint probability density of $V(t)$ and $\dot{V}(t)$. Since $V(t)$ and $\dot{V}(t)$ are assumed to be stationary Gaussian processes, the cross-correlation between them vanishes, $\langle V(t) \dot{V}(t)\rangle=0$. Denote the variance of $V(t)$ and $\dot{V}(t)$ as $\sigma_{V}^{2}$ and $\sigma_{\dot{V}}^{2}$, then the correlation matrix for a point neuron with constant threshold is given by

$$
C=\left(\begin{array}{cc}
\left\langle V(t)^{2}\right\rangle-V_{0}^{2} & \langle V(t) \dot{V}(t)\rangle \\
\langle V(t) \dot{V}(t)\rangle & \left\langle\dot{V}^{2}(t)\right\rangle
\end{array}\right)=\left(\begin{array}{cc}
\sigma_{V}^{2} & 0 \\
0 & \sigma_{\dot{V}}^{2}
\end{array}\right),
$$

where $V_{0}=\langle V(t)\rangle$ is the expected value of membrane potential and we will take $V_{0}=0$. Note that in Naundorf (2005), the voltage threshold was taken as an dynamical variable and the correlation matrix is three dimension. When the spatial 
dimension is included, the correlation matrix will be a higher dimension matrix, depending on the independent random variable included, as will be detailed in the next sections. The probability density for a point neuron is given by

$$
\begin{aligned}
P(V(t), \dot{V}(t)) & =\frac{1}{2 \pi \sqrt{\operatorname{det} C}} \exp \left\{-\frac{1}{2}\left(\begin{array}{c}
V(t) \\
\dot{V}(t)
\end{array}\right)^{T} C^{-1}\left(\begin{array}{c}
V(t) \\
\dot{V}(t)
\end{array}\right)\right\} \\
& =\frac{1}{2 \pi \sigma_{V} \sigma_{\dot{V}}}\left\{-\frac{1}{2}\left(\frac{V(t)^{2}}{\sigma_{V}^{2}}+\frac{\dot{V}^{2}(t)}{\sigma_{\dot{V}}^{2}}\right)\right\} .
\end{aligned}
$$

The integrals in Eq. (5.4) could be performed explicitly and the stationary firing rate is given by

$$
\nu_{0}=\frac{\sigma_{\dot{V}}}{2 \pi \sigma_{V}} \exp \left(-\frac{\psi_{0}^{2}}{2 \sigma_{V}^{2}}\right) .
$$

When a small current $\varepsilon \exp (i \omega t)$ is injected, there will be an filtered voltage $f(t)$ added to the membrane potential which is determined by the subthreshold dynamics, while the membrane voltage is still assumed to be a Gaussian random process. Generally, $f(t)$ is given by

$$
f(t)=\varepsilon \tilde{G}(i \omega) \exp (i \omega t),
$$

where $\tilde{G}(i \omega)$ is the Fourier transform of the Green's function which determines the neuron's response for a $\delta$ pulse input. For a point neuron, $f(t)$ is just the input signal after a low pass filter,

$$
f(t)=\varepsilon \frac{1}{1+i \omega \tau_{m}} \exp (i \omega t) .
$$

Replacing $V(t) \rightarrow V(t)+f(t)$ in Eq. (5.4), the double integral could be performed. Keeping terms till the linear order of $\varepsilon$,

$$
\nu(t)=\nu_{0}+\varepsilon \nu_{1}(\omega) e^{i \omega t} .
$$

where $\nu_{0}$ is given by Eq. (5.7). The response function $\nu_{1}(\omega)$ reads

$$
\begin{aligned}
\nu_{1}(\omega) & =\frac{1}{2 \pi \sigma_{V}} e^{-\frac{\psi_{0}^{2}}{2 \sigma_{V}^{2}}}\left(\frac{\sigma_{\dot{V}} \psi_{0}}{\sigma_{V}^{2}}+\sqrt{\frac{\pi}{2}} i \omega\right) \tilde{G}(i \omega) \\
& =\nu_{0} \frac{\psi_{0}}{\sigma_{V}^{2}}\left(1+i \tau_{e f f} \omega\right) \tilde{G}(i \omega),
\end{aligned}
$$

where

$$
\tau_{e f f}=\sqrt{\frac{\pi}{2}} \frac{\sigma_{V}^{2}}{\psi_{0} \sigma_{\dot{V}}}
$$

is an effective time constant.

For a spatially extended neuron, we will assume that at every point along the axon the membrane potential $V(x, t)$ is a Gaussian random variable and has the above prosperities. The relation between different positions in the axon are determined by the cable equation. 


\subsection{Transfer function for a homogeneous cable}

Now we will determine the Green's functions which tell the voltage at position $x$ along the cable, $V(x, t)$, given the voltage or input current at $x=0$. The soma is taken as a point at $x=0$ and its voltage is denoted as $V_{s}$, i.e., $V_{s}(t) \equiv V(0, t)$. Since we are most interested on the effect of the AIS, which is much shorter than the electrotonic length $\lambda$ at axon, an semi-infinite cable will be adopted.

\subsubsection{Simple ball-and-stick model}

For comparison, we will first ignore the existence of the AIS and the specific properties there, and consider a homogeneous cable in a simple ball-and-stick model. The axon is taken as a semi-infinite homogeneous cable, with the electrotonic length $\lambda$ and membrane time constant $\tau_{m}$. The soma and axon are assumed to have the same membrane time constant. We will take $\lambda$ as the unit of length. By applying the Laplace transform, the cable equation (5.2) becomes

$$
\frac{\partial^{2} V(x, s)}{\partial x^{2}}-\left(1+\tau_{m} s\right) V(x, s)=0 .
$$

Assuming that the voltage at infinity remains finite, there is

$$
V(x, s) \equiv V_{s}(s) \tilde{G}_{1}(x, s)=V_{s} e^{-x \sqrt{\tau_{m} s+1}},
$$

where $\tilde{G}_{1}(x, s)$ is the Laplace transform of the Green's function $G_{1}(x, t)$.

Now taking into account the subthreshold dynamics at soma,

$$
\tau_{m} \frac{d V_{s}}{d t}=-V_{s}+\mu+\frac{1}{A g_{L}} I_{a}
$$

where $A$ is the area of soma and $g_{L}$ is the leak conductance at soma. $\mu(t)$ is a Gaussian random input current with a given correlation function. $I_{a}$ is the lateral inward current into the soma which is proportional to the gradient of the voltage at $x=0$,

$$
I_{a}(t)=\left.\frac{\pi r_{a}^{2}}{r_{L} \lambda} \frac{\partial}{\partial x} V(x, t)\right|_{x=0}
$$

Substituting Eq. (5.16) into Eq. (5.15), we get

$$
\tau_{m} \frac{d V_{s}}{d t}=-V_{s}+\mu+\left.K \frac{\partial}{\partial x} V(x, t)\right|_{x=0}
$$

where $K=\frac{\pi r_{a}^{2}}{A g_{L} r_{L} \lambda}$ is a dimensionless constant. Using the values $r_{m}=1 \mathrm{M} \Omega \cdot \mathrm{mm}^{2}$, $r_{a}=1 \sim 2 \mu \mathrm{m}, r_{L}=1 \mathrm{k} \Omega \cdot \mathrm{mm}, g_{L}=1 \mathrm{M}^{-1} \cdot \mathrm{mm}^{-2}, A=0.01 \sim 0.1 \mathrm{~mm}^{2}$, we 
have $K \sim 1$. But obviously this description is oversimplified. We will take $K$ as a free parameter.

Performing Laplace transform to Eq. (5.17),

$$
\begin{aligned}
\tau_{m} s V_{s}(s) & =-V_{s}(s)+\mu(s)+\left.K \frac{\partial}{\partial x} \tilde{G}_{1}(x, s) V_{s}(s)\right|_{x=0} \\
& =-V_{s}(s)+\mu(s)-K \sqrt{\tau_{m} s+1} V_{s}(s),
\end{aligned}
$$

$V_{s}(s)$ is then determined as a function of input current into the soma,

$$
V_{s}(s)=\frac{1}{\tau_{m} s+1+K \sqrt{\tau_{m} s+1}} \mu(s) .
$$

Correspondingly, from Eq. (5.14),

$$
\begin{aligned}
V(x, s) & \equiv \tilde{G}_{2}(x, s) \mu(s) \\
& =e^{-x \sqrt{\tau_{m} s+1}} \frac{1}{\sqrt{\tau_{m} s+1}\left(\sqrt{\tau_{m} s+1}+K\right)} \mu(s) .
\end{aligned}
$$

To obtain the Green's function in the time domain, the following formulas for Laplace transform will be used

$$
\begin{aligned}
& e^{-b \sqrt{s}} \Rightarrow \frac{b}{2 \sqrt{\pi t^{3}}} \exp \left(-\frac{b^{2}}{4 t}\right), b \geq 0 \\
& \frac{e^{-b \sqrt{s}}}{\sqrt{s}(\sqrt{s}+K)} \Rightarrow e^{K b+K^{2} t} \operatorname{Erfc}\left(K \sqrt{t}+\frac{b}{2 \sqrt{t}}\right), b \geq 0
\end{aligned}
$$

where $\operatorname{Erfc}(x)$ is the complementary error function defined as

$$
\operatorname{Erfc}(x)=\frac{2}{\sqrt{\pi}} \int_{x}^{\infty} e^{-x^{2}} d x
$$

From Eq. (5.14) and Eq. (5.20), we obtain

$$
\begin{aligned}
V(x, t) & =G_{1}(x, t) \otimes V_{s}(t) \\
& =G_{2}(x, t) \otimes \mu(t),
\end{aligned}
$$

where $G_{1}(x, t)$ and $G_{2}(x, t)$ are the corresponding Green's functions in the time domain for a homogeneous cable with a given random function $V_{s}(t)$ and $\mu(t)$ at $x=0$ respectively,

$$
\begin{aligned}
G_{1}(x, t) & =\frac{x}{2 \tau_{m} \sqrt{\pi\left(t / \tau_{m}\right)^{3}}} e^{-t / \tau_{m}} e^{-\tau_{m} x^{2} / 4 t} \\
G_{2}(x, t) & =\frac{1}{\tau_{m}} e^{K x} e^{\left(K^{2}-1\right) t / \tau_{m}} \operatorname{Erfc}\left(K \sqrt{t / \tau_{m}}+\frac{x}{2 \sqrt{t / \tau_{m}}}\right) .
\end{aligned}
$$

Here ' $\otimes$ ' represents convolution, defined as

$$
f_{1}(t) \otimes f_{2}(t)=\int_{0}^{t} f_{1}(t-\tau) f_{2}(\tau) d \tau
$$

Note that $G_{1}(x, t)$ satisfies $\left.G_{1}(x, t)\right|_{x \rightarrow 0}=\delta(t)$. Both $G_{1}(x, t)$ and $G_{2}(x, t)$ decay to zero with the increasing of $x$ and $t$. 


\subsubsection{Two-compartment cable}

To investigate the effect of different physiological properties between the AIS and other parts of the axon, we consider a two-compartment cable, as illustrated in Fig. 5.1A. For the AIS part (region 1, $0 \leq x<x_{1}$ ) and the axon part (region $\left.2, x_{1} \leq x<\infty\right)$, the membrane time constants and electrotonic lengths are $\tau_{1}$, $\lambda_{1}$ and $\tau_{2}, \lambda_{2}$ respectively. At $x=0$ (the soma), there are two parameters $\tau_{m}$ and the coupling constant $K$. The injected current at soma is $\mu(t)$. Note that when $\tau_{1}=\tau_{2}=\tau_{m}$ and $\lambda_{1}=\lambda_{2}$ the two-compartment cable reduces to the simple ball-and-stick model.

In the two regions, the cable equations after applying Laplace transform are

$$
\begin{array}{ll}
\lambda_{1}^{2} \frac{\partial^{2} V(x, s)}{\partial x^{2}}-\left(1+\tau_{1} s\right) V(x, s)=0, & 0 \leq x<x_{1} \\
\lambda_{2}^{2} \frac{\partial^{2} V(x, s)}{\partial x^{2}}-\left(1+\tau_{2} s\right) V(x, s)=0, & x_{1} \leq x<\infty
\end{array}
$$

The boundary conditions are as follows. At $x \rightarrow \infty$, there is $V_{2}(x, s) \rightarrow 0$. At $x=0$, the dynamics is given by Eq. (5.17),

$$
\tau_{m} \frac{d V_{s}}{d t}=-V_{s}+\mu+\left.K \frac{\partial}{\partial x} V_{1}(x, t)\right|_{x=0} .
$$

At $x_{1}$, there are

$$
\begin{aligned}
V_{1}\left(x_{1}, t\right) & =V_{2}(x, t) \\
\frac{\partial}{\partial x} V_{1}(x, t) & =\left.\frac{\partial}{\partial x} V_{2}(x, t)\right|_{x=x_{1}}
\end{aligned}
$$

which reflect the continuity of the membrane potential and the lateral current.

With these boundary conditions, the voltage at a position $x$ along the axon is determined as a function of the input current at the soma. For $0 \leq x<x_{1}$ we have

$$
\begin{aligned}
V_{1}(x, s) \equiv & \mu(s) \tilde{G}_{3}(x, s) \\
= & \mu(s)\left(\sqrt{1+\tau_{2} s} \sinh \left(\frac{x_{1}-x}{\lambda_{1}} \sqrt{1+\tau_{1} s}\right)\right. \\
& \left.\quad+\frac{\lambda_{2}}{\lambda_{1}} \sqrt{1+\tau_{1} s} \cosh \left(\frac{x_{1}-x}{\lambda_{1}} \sqrt{1+\tau_{1} s}\right)\right) \times \\
& \left(\left(\left(1+\tau_{m} s\right) \sqrt{1+\tau_{2} s}+K \frac{\lambda_{2}}{\lambda_{1}}\left(1+\tau_{1} s\right)\right) \sinh \left(\frac{x_{1}}{\lambda_{1}} \sqrt{1+\tau_{1} s}\right)+\right. \\
& \left.\left(\frac{\lambda_{2}}{\lambda_{1}}\left(1+\tau_{m} s\right) \sqrt{1+\tau_{1} s}+K \sqrt{1+\tau_{1} s} \sqrt{1+\tau_{2} s}\right) \cosh \left(\frac{x_{1}}{\lambda_{1}} \sqrt{1+\tau_{1} s}\right)\right)^{-1},
\end{aligned}
$$


from which there is

$$
\begin{aligned}
V_{s}(s)= & \mu(s)\left[\sqrt{1+\tau_{2} s} \sinh \left(\frac{x_{1}}{\lambda_{1}} \sqrt{1+\tau_{1} s}\right)+\frac{\lambda_{2}}{\lambda_{1}} \sqrt{1+\tau_{1} s} \cosh \left(\frac{x_{1}}{\lambda_{1}} \sqrt{1+\tau_{1} s}\right)\right] \\
& \left(\left(\left(1+\tau_{m} s\right) \sqrt{1+\tau_{2} s}+K \frac{\lambda_{2}}{\lambda_{1}}\left(1+\tau_{1} s\right)\right) \sinh \left(\frac{x_{1}}{\lambda_{1}} \sqrt{1+\tau_{1} s}\right)+\right. \\
& \left.\left(\frac{\lambda_{2}}{\lambda_{1}}\left(1+\tau_{m} s\right) \sqrt{1+\tau_{1} s}+K \sqrt{1+\tau_{1} s} \sqrt{1+\tau_{2} s}\right) \cosh \left(\frac{x_{1}}{\lambda_{1}} \sqrt{1+\tau_{1} s}\right)\right)^{-1} .
\end{aligned}
$$

This gives

$$
V_{1}(x, s)=V_{s}(s) \tilde{G}_{4}(x, s)
$$

with

$$
\tilde{G}_{4}(x, s)=\frac{\sqrt{1+\tau_{2} s} \sinh \left(\frac{x_{1}-x}{\lambda_{1}} \sqrt{1+\tau_{1} s}\right)+\frac{\lambda_{2}}{\lambda_{1}} \sqrt{1+\tau_{1} s} \cosh \left(\frac{x_{1}-x}{\lambda_{1}} \sqrt{1+\tau_{1} s}\right)}{\sqrt{1+\tau_{2} s} \sinh \left(\frac{x_{1}}{\lambda_{1}} \sqrt{1+\tau_{1} s}\right)+\frac{\lambda_{2}}{\lambda_{1}} \sqrt{1+\tau_{1} s} \cosh \left(\frac{x_{1}}{\lambda_{1}} \sqrt{1+\tau_{1} s}\right)} .
$$

For $x>x_{1}$, there is

$$
V_{2}(x, s)=V_{s}(s) \tilde{G}_{5}(x, s)
$$

with

$$
G_{5}(x, s)=\frac{\frac{\lambda_{2}}{\lambda_{1}} \sqrt{1+\tau_{1} s} \exp \left(-\frac{x-x_{1}}{\lambda_{2}} \sqrt{1+\tau_{2} s}\right)}{\sqrt{1+\tau_{2} s} \sinh \left(\frac{x_{1}}{\lambda_{1}} \sqrt{1+\tau_{1} s}\right)+\frac{\lambda_{2}}{\lambda_{1}} \sqrt{1+\tau_{1} s} \cosh \left(\frac{x_{1}}{\lambda_{1}} \sqrt{1+\tau_{1} s}\right)} .
$$

For the two-compartment cable, we have obtained the Laplace transform of the Green's functions. Since the Gaussian random field $V(x, t)$ is assumed to be stationary, its stochastic properties can be obtained using the Green's function at Fourier domain by applying the Wiener-Khinchintheorem after the replacement $s \rightarrow i \omega$.

\subsection{Spike-triggered average}

Spike-triggered average provides an important framework for characterizing the response property according to the spike trains of neurons. In Gaussian neuron model the spike-triggered average can be calculated analytically. The theoretical results in Gaussian neuron model are presented through the correlation function $C(\tau)$ and its derivatives, independent of the concrete form of $C(\tau)$. Each choice of the correlation function $C(\tau)$ defines a special kind of model. We will choose the correlation function of $V_{s}(t)$, the voltage at soma, as a difference of two exponentials,

$$
C_{V s}(\tau)=\left\langle V_{s}(t) V_{s}(t+\tau)\right\rangle=\frac{\sigma_{V}^{2}}{\tau_{D}-\tau_{R}}\left(\tau_{D} e^{-\frac{|\tau|}{\tau_{D}}}-\tau_{R} e^{-\frac{|\tau|}{\tau_{R}}}\right)
$$


This correlation function is two times differentiable at $\tau=0$. It describes the correlation function of a white noise after two times filtering, a slow decay with time constant $\tau_{D}$ after a fast rising period with time constant $\tau_{R}$ mimicking the realistic synaptic filtering. When $\tau_{D}=\tau_{R}=\tau$, the correlation function takes the form

$$
C_{V s}(\tau)=\sigma_{V}^{2}\left(1+\frac{|\tau|}{\tau_{D}}\right) e^{-\frac{|\tau|}{\tau_{D}}}
$$

The correlation function of $\dot{V}_{s}(t)$ is obtained as

$$
C_{\dot{V}_{s}}(\tau)=-C_{V_{s}}^{\prime \prime}(\tau)=\frac{\sigma_{V}^{2}}{\tau_{D}-\tau_{R}}\left(\frac{1}{\tau_{R}} e^{-\frac{|\tau|}{\tau_{R}}}-\frac{1}{\tau_{D}} e^{-\frac{|\tau|}{\tau_{D}}}\right)
$$

For $\tau=0$, there are $C_{V_{s}}(0)=\sigma_{V}^{2}$ and $C_{\dot{V}_{s}}(0)=\frac{\sigma_{V}^{2}}{\tau_{D} \tau_{R}} \equiv \sigma_{\dot{V}}^{2}$. The power spectral is given by

$$
P_{s}(w)=\frac{2 \sigma_{V}^{2}}{\tau_{D}-\tau_{R}}\left(\frac{\tau_{D}^{2}}{1+\tau_{D}^{2} \omega^{2}}-\frac{\tau_{R}^{2}}{1+\tau_{R}^{2} \omega^{2}}\right)
$$

In performing numerical evaluation to visualize the results, we use the following parameters: $\tau_{m}=10 \mathrm{~ms}, \tau_{D}=10 \mathrm{~ms}, \tau_{R}=1 \mathrm{~ms}$ and $\sigma_{V}=5 \mathrm{mV}$. The constant threshold is $\psi_{0}=10 \mathrm{mV}$. Fig. $5.1 \mathrm{C}$ is the correlation function of the membrane potential at the soma, as given by Eq. (5.38). Three trajactories of the membrane potential at the soma, the middle of the AIS and the end of the AIS are shown in Fig. 5.1D.

Given the correlation function at $x=0$, the stationary covariance of $V(x, t)$ and $\dot{V}(x, t)$ at position $x$ can be expressed in the Fourier domain by implementing the Green's function obtained in the previous section,

$$
\begin{aligned}
C_{V x}(\tau) & =\langle V(x, t) V(x, t+\tau)\rangle \\
& =\frac{1}{2 \pi} \int_{-\infty}^{\infty} d \omega e^{i \omega \tau} P_{s}(\omega)|\tilde{G}(i \omega)|^{2} \\
C_{\dot{V} x}(\tau) & =-C_{V x}^{\prime \prime}(\tau) \\
& =\frac{1}{2 \pi} \int_{-\infty}^{\infty} d \omega e^{i \omega \tau} \omega^{2} P_{s}(\omega)|\tilde{G}(i \omega)|^{2},
\end{aligned}
$$

where $\tilde{G}(i \omega)$ is the Fourier transform of the Green's function (obtained by replacing $s$ with $i \omega$ in the Laplace transform of the Green's function). For the simple ball-and-stick model and two-compartment cable, it represents $\tilde{G}_{1}$ and $\tilde{G}_{4}$ respectively. When $\tau=0$, Eq. (5.42) gives $\sigma_{V x}^{2}$ and $\sigma_{\dot{V} x}^{2}$, the variance of $V(x, t)$ and $\dot{V}(x, t)$.

The covariance $\left\langle V_{s}(t-\tau) V(x, t)\right\rangle$ and $\left\langle V_{s}(t-\tau) \dot{V}(x, t)\right\rangle$ can also be expressed 
in a similar way through the Green's function by the cross-correlation theorem,

$$
\begin{aligned}
C_{s V x}(\tau) & =\left\langle V_{s}(t-\tau) V(x, t)\right\rangle \\
& =\frac{1}{2 \pi} \int_{-\infty}^{\infty} d \omega e^{i \omega \tau} P_{s}(\omega) \tilde{G}(i \omega) \\
C_{s \dot{V} x}(\tau) & =\left\langle V_{s}(t-\tau) \dot{V}(x, t)\right\rangle \\
& =\frac{1}{2 \pi} \int_{-\infty}^{\infty} d \omega e^{i \omega \tau} i \omega P_{s}(\omega) \tilde{G}(i \omega)
\end{aligned}
$$

For the ball-and-stick model, the integrals in Eq. (5.43) can be performed explicitly. We will present the results in the Appendix, together with the small $x$ approxmation for the covariance Eq. (5.42).

In Gaussian neuron model, the spike-triggered average voltage and variance are defined as

$$
\begin{aligned}
\bar{V}_{s}(\tau, x)= & \frac{1}{\nu_{0 x}}\left\langle V_{s}(t-\tau) \delta\left(V_{x}(t)-\psi_{0}\right)\left|\dot{V}_{x}(t)\right| \theta\left(\dot{V}_{x}(t)\right)\right\rangle \\
= & \frac{1}{\nu_{0 x}} \iiint_{-\infty}^{\infty} d V_{s}(t-\tau) d \dot{V}_{x}(t) d V_{x}(t) V_{s}(t-\tau) \delta\left(V_{x}(t)-\psi_{0}\right)\left|\dot{V}_{x}(t)\right| \\
& \times \theta\left(\dot{V}_{x}(t)\right) P_{S T V}\left(V_{s}(t-\tau), \dot{V}_{x}(t), V_{x}(t)\right), \\
S T V(\tau, x)= & \frac{1}{\nu_{0 x}}\left\langle\left(V_{s}(t-\tau)-\bar{V}_{s}(\tau, x)\right)^{2} \delta\left(V_{x}(t)-\psi_{0}\right)\left|\dot{V}_{x}(t)\right| \theta\left(\dot{V}_{x}(t)\right)\right\rangle \\
= & \frac{1}{\nu_{0 x}} \iiint_{-\infty}^{\infty} d V_{s}(t-\tau) d \dot{V}_{x}(t) d V_{x}(t) V_{s}^{2}(t-\tau) \delta\left(V_{x}(t)-\psi_{0}\right)\left|\dot{V}_{x}(t)\right| \\
& \times \theta\left(\dot{V}_{x}(t)\right) P_{S T V}\left(V_{s}(t-\tau), V_{s}(t), \dot{V}_{x}(t), V_{x}(t)\right)-\bar{V}_{s}^{2}(x),
\end{aligned}
$$

where $\nu_{0 x}$ is the stationary firing rate at position $\mathrm{x}$,

$$
\nu_{0 x}=\frac{\sigma_{\dot{V} x}}{2 \pi \sigma_{V x}} e^{-\frac{\psi_{0}^{2}}{2 \sigma_{V x}^{2}}} .
$$

$P_{S T V}\left(V_{s}(t-\tau), \dot{V}_{x}(t), V_{x}(t)\right)$ is the probability density function defined by

$$
\begin{aligned}
& P_{S T V}\left(V_{s}(t-\tau), \dot{V}_{x}(t), V_{x}(t)\right)= \\
& \frac{1}{(2 \pi)^{3 / 2} \sqrt{\operatorname{det} C(\tau, x)}} \exp \left\{-\frac{1}{2}\left(\begin{array}{c}
V_{s}(t-\tau) \\
\dot{V}_{x}(t) \\
V_{x}(t)
\end{array}\right)^{T} C^{-1}(\tau, x)\left(\begin{array}{c}
V_{s}(t-\tau) \\
\dot{V}_{x}(t) \\
V_{x}(t)
\end{array}\right)\right\} .
\end{aligned}
$$


The correlation matrix $C(\tau, x)$ is defined as

$$
\begin{aligned}
C(\tau, x) & =\left(\begin{array}{ccc}
\left\langle V_{s}^{2}(t-\tau)\right\rangle & \left\langle V_{s}(t-\tau) \dot{V}_{x}(t)\right\rangle & \left\langle V_{s}(t-\tau) V_{x}(t)\right\rangle \\
\left\langle V_{s}(t-\tau) \dot{V}_{x}(t)\right\rangle & \left\langle\dot{V}_{x}^{2}(t)\right\rangle & \left\langle\dot{V}_{x}(t) V_{x}(t)\right\rangle \\
\left\langle V_{s}(t) V_{x}(t)\right\rangle & \left\langle\dot{V}_{x}(t) V_{x}(t)\right\rangle & \left\langle V_{x}^{2}(t)\right\rangle
\end{array}\right) \\
& =\left(\begin{array}{ccc}
\sigma_{V}^{2} & C_{s \dot{V} x}(\tau) & C_{s V x}(\tau) \\
C_{s \dot{V} x}(\tau) & \sigma_{\dot{V} x}^{2} & 0 \\
C_{s V x}(\tau) & 0 & \sigma_{V x}^{2}
\end{array}\right) .
\end{aligned}
$$

The determinant of $C(\tau, x)$ is

$$
\operatorname{Det}(C(\tau, x))=\sigma_{V}^{2} \sigma_{\dot{V} x}^{2} \sigma_{V x}^{2}-C_{s \dot{V} x}(\tau) \sigma_{V x}^{2}-C_{s V x}(\tau) \sigma_{\dot{V} x}^{2}
$$

where the first and third terms have very close values and the contribution from the second term is small compared with other two terms.

Performing the double integration, we obtain

$$
\begin{aligned}
\bar{V}_{s}(\tau, x)= & \frac{1}{\nu_{0 x}} \frac{1}{2 \pi \sigma_{V x}^{3}} \exp \left(-\frac{\psi_{0}^{2}}{2 \sigma_{V x}^{2}}\right)\left(\sqrt{\frac{\pi}{2}} \sigma_{V x}^{2} C_{s \dot{V} x}(\tau)+\psi_{0} \sigma_{\dot{V} x} C_{s V x}(\tau)\right) \\
= & \sqrt{\frac{\pi}{2}} \sigma_{\dot{V} x}^{-1} C_{s \dot{V} x}(\tau)+\psi_{0} \sigma_{V x}^{-2} C_{s V x}(\tau) \\
S T V(\tau, x)= & \frac{1}{\nu_{0 x}} \frac{1}{2 \pi \sigma_{\dot{V} x} \sigma_{V x}^{5}} \exp \left(-\frac{\psi_{0}^{2}}{2 \sigma_{V x}^{2}}\right)\left(\sigma_{\dot{V} x}^{2}\left(\psi_{0}^{2}-\sigma_{V x}^{2}\right) C_{s V x}^{2}(\tau)+\right. \\
& \left.\sqrt{2 \pi} \psi_{0} \sigma_{\dot{V} x} \sigma_{V x}^{2} C_{s V x}(\tau) C_{s \dot{V} x}(\tau)+\sigma_{V x}^{4}\left(\sigma_{V}^{2} \sigma_{\dot{V} x}^{2}+C_{s \dot{V} x}^{2}(\tau)\right)\right)-\bar{V}_{s}^{2}(x) \\
= & \sigma_{V}^{2}-\sigma_{V x}^{-2} C_{s V x}^{2}(\tau)-\left(\sqrt{\frac{\pi}{2}}-1\right) \sigma_{\dot{V} x}^{-2} C_{s \dot{V} x}^{2}(\tau) .
\end{aligned}
$$

Fig. 5.2 shows the variation of the spike-triggered voltage and spike-triggered variance with $\mathrm{x}$ at the spiking time $(\tau=0)$ for different membrane time constant $\tau_{1}$ at the AIS assuming the AIS and axon have similar electrotonic length. The membrane time constant at the AIS does not have a significant influence on the spike-triggered average. Since the time delay for observing AP at soma is quite short ( about 0.1ms, see e.g., Palmer \& Stuart (2006)), the spike-triggered variance measures the variation of the threshold for AP generation at soma. From Fig. $5.2 \mathrm{~B}$, the STV is only about 1 percent of the variance of membrane potential at soma. So the STV can explains about 10-20 percent of the threshold variability at soma, which suggests that the experimentally observed large threshold variability in Naundorf et al. (2006) reflects an intrinsic feature in the AP generator. Note that from Eq. (5.51) the spike-triggered variance does not depend on the choice of voltage threshold. 
A

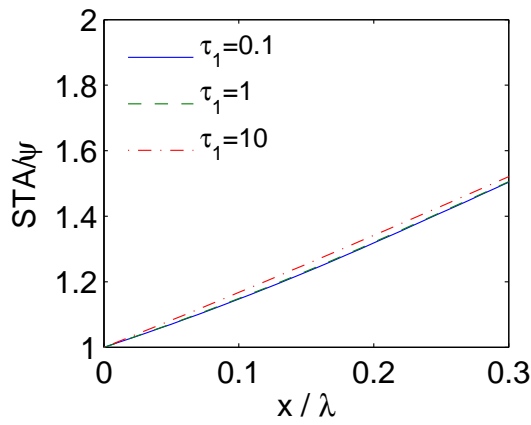

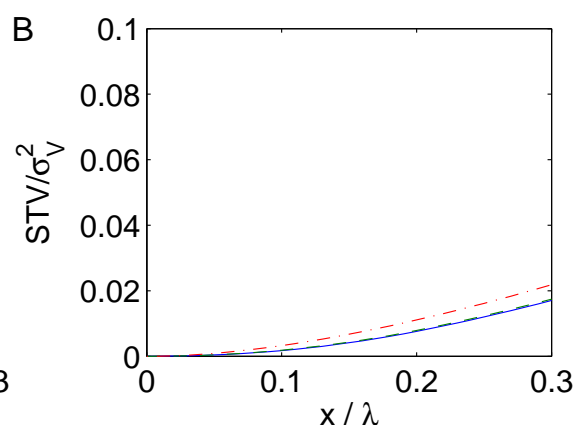

Figure 5.2: Dependence of the spike-triggered average voltage (A) and variance (B) at soma on the position of AP evoked along the axon. Note that $\tau_{s}=10 \mathrm{~ms}$ corresponds to the simple ball-and-stick model. Parameters used: $\psi_{0}=10$, $\frac{\lambda_{2}}{\lambda_{1}}=1 . x_{1}=0.2 \lambda_{1}$.

\subsection{Linear response}

With the expressions for the Green's functions and the covariances along the axon, the linear response at a position $\mathrm{x}$ where an AP is initiated can be obtained from Eq. (5.11).

\subsubsection{Two coupled Gaussian neurons}

Before investigating the linear response in a spatial extended neuron, we first check a simpler system composed of two coupled point Gaussian neurons (or two coupled point-like compartments), in which only one neuron receives input signals and the APs are initiated at the other neuron. The two neurons connect with each other through a coupling strength $c$. They have different membrane time constants. This is a simplified system for the axonal AP initiation we are studying. The neuron which receives external input has a given correlation function, say, Eq. (5.38). In the Appendix, we derive the linear response analytically. A shorter membrane time constant at the AP initiation part has the effect of increasing the cutoff frequency for dynamical signal encoding, as can be seen from Fig. 5.3. A stronger coupling between the two neurons also leads to enhancement of high frequency response. Similar effects are expected to be observed in the two-compartment axon.

\subsubsection{Two-compartment cable}

For a two-compartment cable, the effect of the AIS initiation of APs can be studied. The simple ball-and-stick model is included here as a specific case when $\tau_{1}=\tau_{2}=\tau_{m}$ and $\lambda_{1}=\lambda_{2}$. 

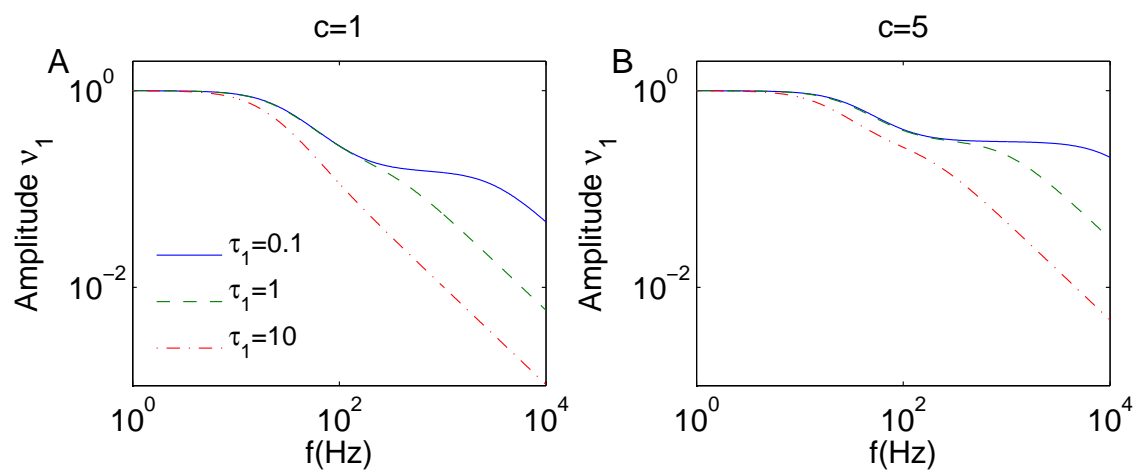

Figure 5.3: Dependence of transmission function on the membrane time constant $\tau_{1}$ at the AP initiation compartment for different coupling constant $c$. A, $c=1$; $\mathrm{B}, c=5$.

When a small signal $\varepsilon e^{i \omega t}$ is injected at soma, the filtered signal felt at position $x$ in the AIS is given by

$$
f(x, t)=\varepsilon \tilde{G}_{3}(x, i \omega) e^{i \omega t} .
$$

The covariances at $x$ are given by Eq. (5.42,5.43), with the Green's function changed to $\tilde{G}_{4}(x, i \omega)$. The variances of $V(x, t)$ and $\dot{V}(x, t)$ are then given by $\sigma_{V x}^{2}=C_{V x}(0)$ and $\sigma_{\dot{V} x}^{2}=C_{\dot{V} x}(0)$. The linear response at position $x$ then has the following form

$$
\begin{aligned}
\nu_{1 x}(\omega) & =\frac{1}{2 \pi \sigma_{V x}} e^{-\frac{\psi_{0}^{2}}{2 \sigma_{V x}^{2}}}\left(\frac{\sigma_{\dot{V} x} \psi_{0}}{\sigma_{V x}^{2}}+\sqrt{\frac{\pi}{2}} i \omega\right) \tilde{G}_{3}(x, i \omega) \\
& =\nu_{0 x} \frac{\psi_{0}}{\sigma_{V x}^{2}}\left(1+i \tau_{e f f} \omega\right) \tilde{G}_{3}(x, i \omega)
\end{aligned}
$$

where

$$
\tau_{e f f}=\sqrt{\frac{\pi}{2}} \frac{\sigma_{V x}^{2}}{\psi_{0} \sigma_{\dot{V} x}}
$$

is an effective time constant.

From Fig. 5.4 and 5.5, we see that compared with a homogeneous axon, the high frequency response is enhanced strongly when the membrane time constant at the AIS is shorter than soma and axon and a stronger coupling constant between soma and AIS increases the normalized response further. The linear response does no change much when the AP initiation position moves from the middle of the AIS to the end of it. Note that the absolute response amplitude at zero frequency decrease with the coupling constant as $1 / K$, as can be seen from the Green's function. This is different from the coupled Gaussian neurons, where the response at zero frequency approaches to a limit value independence of the coupling constant $c$ for $c \rightarrow \infty$. 

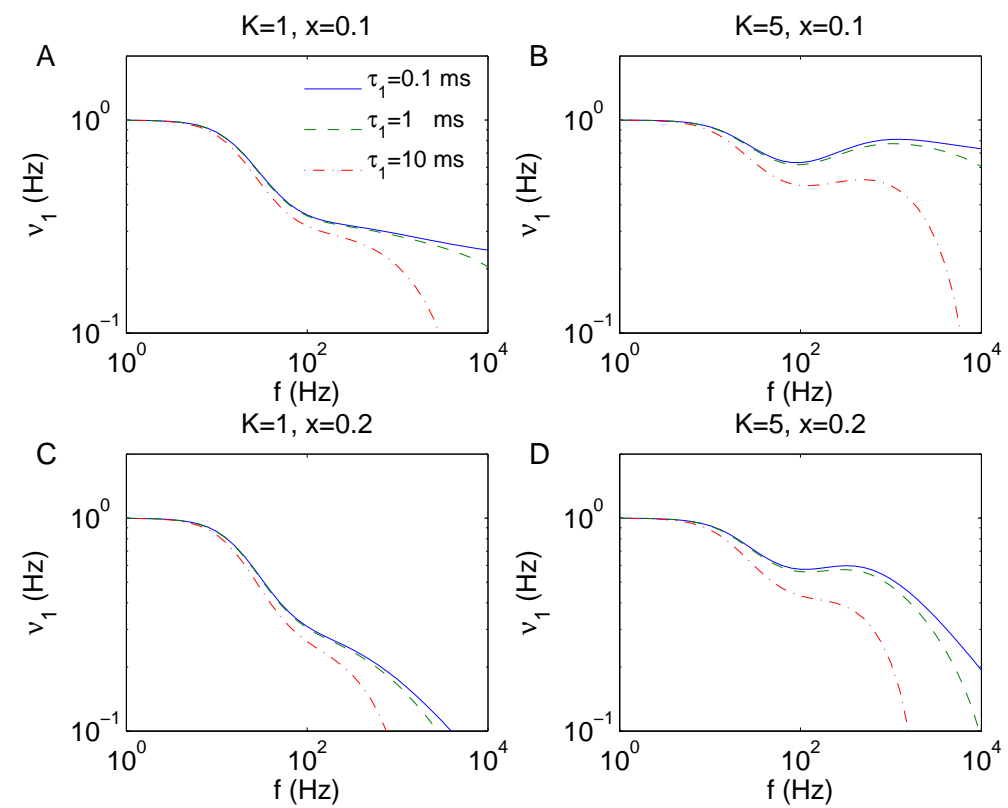

Figure 5.4: Dependence of transmission functions on the time constant $\tau_{1}$ at AP initiation compartment for different coupling constant $K$ and at different position of the AIS. For A and B, $x=0.1$, in the middle of the AIS; C and D, $x=0.2$, at the end of AIS. Other parameters used are: $\tau_{2}=\tau_{m}=10 \mathrm{~ms}, \lambda_{2} / \lambda_{1}=1$.
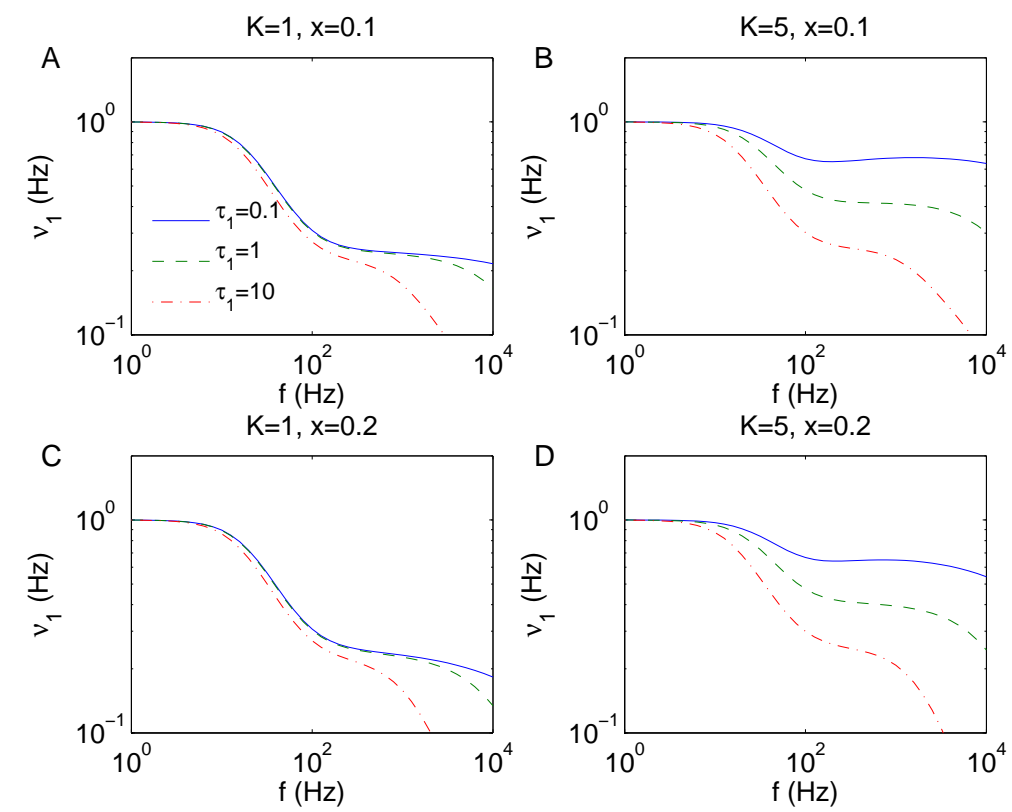

Figure 5.5: The same as Fig. 5.4, but with $\lambda_{2} / \lambda_{1}=10$. 


\subsubsection{Relation between spike-triggered average and linear response}

The spike-triggered average voltage is the linear term in the voltera expansion of the neuron's response and is therefore related to the linear response. In fact the linear response can be readily obtained from the spike triggered average voltage. The Fourier transform of $\bar{V}_{s}(x, \tau)$ normalized by the power spectrum $P_{V_{s}}(\omega)$ give the linear response to a small sinusoidal component in the soma voltage, which is related to a small soma injected current by $\tilde{G}_{3}(0, i \omega)$. We then have

$$
\nu_{1}(\omega)=\nu_{0 x} \tilde{\bar{V}}_{s}(i \omega, x) \tilde{G}_{3}(0, i \omega) / P_{V_{s}}(\omega)
$$

which reproduces exactly the linear response result Eq. (5.53). Note that the factor $\nu_{0 x}$ is from the normalization we have used in the definition of the spiketriggered average voltage. This proves the equivalency between the linear reversecorrelation approach and the linear response in the Gaussian neuron model.

\subsection{Discussion}

Two aspects about the possible functional role of AP initiation at the AIS were checked in this chapter. First we addressed the question whether it exhibits a strong influence on the threshold variability of somatic AP generation. Here we calculated the spike-triggered average analytically in the Gaussian neuron model. The spike-triggered variance constitutes only a small fraction of the variability of the voltage at the soma. From extensive simulation studies (Huang, 2009), the conditions that axonal AP initiation can have significant influence on the somatic AP waveform was found previously. A long passive dendrite and an extremely high sodium channel density at the AIS seems to be essential for reproducing the key features observed at the soma, which seems not fulfilled in cortical neurons (Fleidervish et al., 2010). This suggests that the characteristic features from recording at the soma reflect intrinsic properties of the cortical AP generation process. Secondly, the linear response of APs generated at the AIS was studied in the Gaussian neuron model. The membrane time constant at the AIS could be different from axon and soma. A shorter membrane time constant at the AIS was shown to enable a faster high frequency response compared with the homogeneous axon. Similarly, a response enhancement was found if the electrotonic length is chosen much longer at the AIS than in the axon. If the longitudinal resistance is assumed to be equal, then this reflects the influence of the membrane resistance. The chandelier cells specifically targeted at the AIS of pvramidal neurons (Howard et al., 2005). One characteristic feature of chandelier cells is its terminals usually climbing upwards along the AIS and targeted at a large range of the AIS of pyramidal neurons, not just a point (Somogyi et al., 1982). These terminals thus can modulate the passive membrane conductance of the AIS effectively, which 
can change the characteristic temporal and spatial scales at the AIS and therefore effectively modulate the linear response behavior of cortical neurons to dendritic and somatic synaptic integration. The physiological properties at the AIS have not been characterized completely yet and many controversies persist, due to the difficulty of directly recording from this small compartment. In principle, however, the passive properties of the AIS could be significantly different from the soma. For example, the low threshold for AP initiation at the AIS was found to be resulted from a higher resting potential at the AIS than at the soma (Kole \& Stuart, 2008). Investigating the influence of these difference on signal encoding is an important further direction for research. 


\subsection{Appendix}

\section{Results for the simple ball-and-stick model}

According to Eq. (5.24), the expected value of $V(x, t)$ with the voltage at soma a given gaussian random function is

$$
\begin{aligned}
&\langle V(x, t)\rangle=\left\langle V_{s}(t)\right\rangle \int_{0}^{t} \frac{x}{2 \sqrt{\pi\left(t^{\prime} / \tau_{m}\right)^{3}}} e^{-t^{\prime} / \tau_{m}} e^{-\tau_{m} x^{2} / 4 t^{\prime}} d t^{\prime} / \tau_{m} \\
&=\frac{1}{2}\left\langle V_{s}(t)\right\rangle\left(e^{-x} \operatorname{Erfc}\left(\frac{x}{2 \sqrt{t / \tau_{m}}}-\sqrt{t / \tau_{m}}\right)\right. \\
&\left.+e^{x} \operatorname{Erfc}\left(\frac{x}{2 \sqrt{t / \tau_{m}}}+\sqrt{t / \tau_{m}}\right)\right) .
\end{aligned}
$$

When the subthreshold dynamics of the soma is included, characterized by Eq. (5.17), the expected value of $V(x, t)$ is given by

$$
\langle V(x, t)\rangle=\mu_{0} \int_{0}^{t} e^{K x} e^{\left(K^{2}-1\right) t^{\prime} / \tau_{m}} \operatorname{Erfc}\left(K \sqrt{t^{\prime} / \tau_{m}}+\frac{x}{2 \sqrt{t^{\prime} / \tau_{m}}}\right) d t^{\prime} / \tau_{m}
$$

where $\mu_{0}=\langle\mu(t)\rangle$ is the expected of the input current. The above integral can be performed explicitly. For $K \neq 1$,

$$
\begin{aligned}
\langle V(x, t)\rangle / \mu_{0}= & \frac{1}{K^{2}-1} e^{K x} e^{\left.\left(K^{2}-1\right)\right) t / \tau_{m}} \operatorname{Erfc}\left(K \sqrt{t / \tau_{m}}+\frac{x}{2 \sqrt{t / \tau_{m}}}\right) \\
& +\frac{1}{2} \frac{1}{K+1} e^{-x} \operatorname{Erfc}\left(\frac{x}{2 \sqrt{t / \tau_{m}}}-\sqrt{t / \tau_{m}}\right) \\
& -\frac{1}{2} \frac{1}{K-1} e^{x} \operatorname{Erfc}\left(\frac{x}{2 \sqrt{t / \tau_{m}}}+\sqrt{t / \tau_{m}}\right) .
\end{aligned}
$$

For $K=1$,

$$
\begin{aligned}
\langle V(x, t)\rangle / \mu_{0}= & \frac{1}{4}\left(e^{-x} \operatorname{Erfc}\left(\frac{x}{2 \sqrt{t / \tau_{m}}}-\sqrt{t / \tau_{m}}\right)\right. \\
& \left.-\left(1-2 x-4 t / \tau_{m}\right) e^{x} \operatorname{Erfc}\left(\frac{x}{2 \sqrt{t / \tau_{m}}}+\sqrt{t / \tau_{m}}\right)\right) \\
& -\sqrt{\frac{t}{\pi \tau_{m}}} e^{-t / \tau_{m}-\frac{x^{2}}{4 t / \tau_{m}}} .
\end{aligned}
$$

The stationary value is

$$
\left.\langle V(x, t)\rangle\right|_{t \rightarrow \infty}=\frac{\mu_{0}}{K+1} e^{-x}
$$


Taking $x=0$ in Eq. (5.58) and Eq.(5.59), we obtain the average voltage at soma,

$$
\left\langle V_{s}(t)\right\rangle / \mu_{0}=\frac{1}{K^{2}-1}\left(e^{\left(K^{2}-1\right) t / \tau_{m}} \operatorname{Erfc}\left(K \sqrt{t / \tau_{m}}\right)-K \operatorname{Erfc}\left(\sqrt{t / \tau_{m}}\right)\right)+\frac{1}{K+1}
$$

for $K \neq 1$ and

$$
\left\langle V_{s}(t)\right\rangle / \mu_{0}=\frac{1}{2}-\left(\frac{1}{2}-t / \tau_{m}\right) \operatorname{Erfc}\left(\sqrt{t / \tau_{m}}\right)-\sqrt{\frac{t}{\pi \tau_{m}}} e^{-t / \tau_{m}}
$$

for $K=1$.

$\left\langle V_{s}(t) V(x, t)\right\rangle$ can also be calculated directly using the Green's function,

$$
\begin{aligned}
\left\langle V_{s}(t) V(x, t)\right\rangle= & \int_{0}^{t} \frac{x}{2 \sqrt{\pi\left(\left(t-t^{\prime}\right) / \tau_{m}\right)^{3}}} \exp \left(-\frac{t-t^{\prime}}{\tau_{m}}-\frac{\tau_{m} x^{2}}{4\left(t-t^{\prime}\right)}\right)\left\langle V_{s}\left(t^{\prime}\right) V_{s}(t)\right\rangle d t^{\prime} / \tau_{m} \\
= & \frac{\sigma_{V}^{2}}{\tau_{D}-\tau_{R}} \frac{\tau_{D}}{2} \times \\
& \left(\exp \left(-x\left(1+\frac{\tau_{m}}{\tau_{D}}\right)^{\frac{1}{2}}\right) \operatorname{Erfc}\left(\frac{x}{2 \sqrt{t / \tau_{m}}}-\sqrt{t / \tau_{m}}\left(1+\frac{\tau_{m}}{\tau_{D}}\right)^{\frac{1}{2}}\right)\right. \\
& \left.+\exp \left(x\left(1+\frac{\tau_{m}}{\tau_{D}}\right)^{\frac{1}{2}}\right) \operatorname{Erfc}\left(\frac{x}{2 \sqrt{t / \tau_{m}}}+\sqrt{t / \tau_{m}}\left(1+\frac{\tau_{m}}{\tau_{D}}\right)^{\frac{1}{2}}\right)\right) \\
& -\left(\tau_{D} \leftrightarrow \tau_{R}\right) .
\end{aligned}
$$

When $t \rightarrow \infty$, we get the stationary variance,

$$
\left\langle V_{s}(t) V(x, t)\right\rangle \rightarrow \frac{\sigma_{V}^{2}}{\tau_{D}-\tau_{R}}\left(\tau_{D} \exp \left(-x\left(1+\frac{\tau_{m}}{\tau_{D}}\right)^{\frac{1}{2}}\right)-\tau_{R} \exp \left(-x\left(1+\frac{\tau_{m}}{\tau_{R}}\right)^{\frac{1}{2}}\right)\right)
$$

The linear approximation for the correlation function Eq. (5.42) can be obtained analytically. For small x, Eq. (5.42) becomes

$$
C_{V x}(\tau) \approx \frac{1}{2 \pi} \int_{-\infty}^{\infty} d \omega e^{i \omega \tau} P^{s}(\omega)\left(1-x\left(\sqrt{1+i \tau_{m} \omega}+\sqrt{1-i \tau_{m} \omega}\right)\right)
$$

For the small $\mathrm{x}$ approximation to be correct, there exists a cutoff frequency $\omega_{c}=$ $\frac{1}{x^{2} \tau_{m}}$ and contribution from frequency higher than $\omega_{c}$ should be ignorable.

The first term in the above equation give just Eq. (5.38), the covariance at the soma. The contribution from the second and third terms can be calculated 
by utilizing the following Fourier transformation formulas

$$
\begin{aligned}
& \frac{1}{2 \pi} \int_{-\infty}^{\infty} d \omega e^{i \omega t} \frac{\sqrt{i \omega+\rho}}{i \omega+\beta} \\
& =\frac{1}{\sqrt{\pi t}} e^{-\rho t}+(\rho-\beta)^{\frac{1}{2}} e^{-\beta t} \operatorname{Erf}\left((\rho-\beta)^{\frac{1}{2}}(t)^{\frac{1}{2}}\right), t>0 \\
& \frac{1}{2 \pi} \int_{-\infty}^{\infty} d \omega e^{i \omega t} \frac{\sqrt{i \omega+\rho}}{-i \omega+\beta} \\
& =\left\{\begin{array}{l}
-\frac{1}{\sqrt{\pi t}} e^{-\rho t}+(\rho+\beta)^{\frac{1}{2}} e^{\beta t} \operatorname{Erfc}\left((\rho+\beta)^{\frac{1}{2}}(t)^{\frac{1}{2}}\right), t>0 \\
(\rho+\beta)^{\frac{1}{2}} e^{\beta t}, t<0
\end{array}\right.
\end{aligned}
$$

where $\operatorname{Re} \rho \geq 0$ and $\operatorname{Re} \beta>0$. Eq. (5.66) can be found in Campbell \& Foster (1961). These formulas can be obtained by contour integration where the pole and branch cut both can have contribution to the integration.

Substituting these formulas into Eq. (5.65) we get

$$
\begin{aligned}
& C_{V x}(\tau) \approx \frac{\sigma_{V}^{2}}{\tau_{D}-\tau_{R}}\left(\tau_{D} \exp \left(-\frac{|\tau|}{\tau_{D}}\right)-\tau_{R} \exp \left(-\frac{|\tau|}{\tau_{R}}\right)\right) \\
& -x \frac{\sigma_{V}^{2}}{\tau_{D}-\tau_{R}}\left(\tau_{D}\left(1-\frac{\tau_{m}}{\tau_{D}}\right)^{\frac{1}{2}} e^{-\frac{|\tau|}{\tau_{D}}} \operatorname{Erf}\left(\left(1-\frac{\tau_{m}}{\tau_{D}}\right)^{\frac{1}{2}}\left|\frac{\tau}{\tau_{m}}\right|^{\frac{1}{2}}\right)\right. \\
& +\tau_{D}\left(1+\frac{\tau_{m}}{\tau_{D}}\right)^{\frac{1}{2}} e^{\frac{|\tau|}{\tau_{D}}} \operatorname{Erfc}\left(\left(1+\frac{\tau_{m}}{\tau_{D}}\right)^{\frac{1}{2}}\left|\frac{\tau}{\tau_{m}}\right|^{\frac{1}{2}}\right) \\
& -\tau_{R}\left(1-\frac{\tau_{m}}{\tau_{R}}\right)^{\frac{1}{2}} e^{-\frac{|\tau|}{\tau_{R}}} \operatorname{Erf}\left(\left(1-\frac{\tau_{m}}{\tau_{R}}\right)^{\frac{1}{2}}\left|\frac{\tau}{\tau_{m}}\right|^{\frac{1}{2}}\right) \\
& -\tau_{R}\left(1+\frac{\tau_{m}}{\tau_{R}}\right)^{\frac{1}{2}} e^{\frac{|\tau|}{\tau_{R}}} \operatorname{Erfc}\left(\left(1+\frac{\tau_{m}}{\tau_{R}}\right)^{\frac{1}{2}}\left|\frac{\tau}{\tau_{m}}\right|^{\frac{1}{2}}\right) \\
& \left.+\tau_{D}\left(1+\frac{\tau_{m}}{\tau_{D}}\right)^{\frac{1}{2}} e^{-\frac{|\tau|}{\tau_{D}}}-\tau_{R}\left(1+\frac{\tau_{m}}{\tau_{R}}\right)^{\frac{1}{2}} e^{-\frac{|\tau|}{\tau_{R}}}\right), \\
& C_{\dot{V} x}(\tau)=-C_{V x}^{\prime \prime}(\tau) \\
& \approx \frac{\sigma_{V}^{2}}{\tau_{D}-\tau_{R}}\left(\frac{1}{\tau_{R}} \exp \left(-\frac{|\tau|}{\tau_{R}}\right)-\frac{1}{\tau_{D}} \exp \left(-\frac{|\tau|}{\tau_{D}}\right)\right) \\
& +x \frac{\sigma_{V}^{2}}{\tau_{D}-\tau_{R}}\left(\frac{1}{\tau_{D}}\left(1-\frac{\tau_{m}}{\tau_{D}}\right)^{\frac{1}{2}} e^{-\frac{|\tau|}{\tau_{D}}} \operatorname{Erf}\left(\left(1-\frac{\tau_{m}}{\tau_{D}}\right)^{\frac{1}{2}}\left|\frac{\tau}{\tau_{m}}\right|^{\frac{1}{2}}\right)\right. \\
& +\frac{1}{\tau_{D}}\left(1+\frac{\tau_{m}}{\tau_{D}}\right)^{\frac{1}{2}} e^{\frac{|\tau|}{\tau_{D}}} \operatorname{Erfc}\left(\left(1+\frac{\tau_{m}}{\tau_{D}}\right)^{\frac{1}{2}}\left|\frac{\tau}{\tau_{m}}\right|^{\frac{1}{2}}\right) \\
& -\frac{1}{\tau_{R}}\left(1-\frac{\tau_{m}}{\tau_{R}}\right)^{\frac{1}{2}} e^{-\frac{|\tau|}{\tau_{R}}} \operatorname{Erf}\left(\left(1-\frac{\tau_{m}}{\tau_{R}}\right)^{\frac{1}{2}}\left|\frac{\tau}{\tau_{m}}\right|^{\frac{1}{2}}\right) \\
& -\frac{1}{\tau_{R}}\left(1+\frac{\tau_{m}}{\tau_{R}}\right)^{\frac{1}{2}} e^{\frac{|\tau|}{\tau_{R}}} \operatorname{Erfc}\left(\left(1+\frac{\tau_{m}}{\tau_{R}}\right)^{\frac{1}{2}}\left|\frac{\tau}{\tau_{m}}\right|^{\frac{1}{2}}\right) \\
& \left.+\frac{1}{\tau_{D}}\left(1+\frac{\tau_{m}}{\tau_{D}}\right)^{\frac{1}{2}} e^{-\frac{|\tau|}{\tau_{D}}}-\frac{1}{\tau_{R}}\left(1+\frac{\tau_{m}}{\tau_{R}}\right)^{\frac{1}{2}} e^{-\frac{|\tau|}{\tau_{R}}}\right) .
\end{aligned}
$$


Expanding $C_{\dot{V} x}(\tau)$ for small $\tau$ we find the time giving the maxim value is $\tau_{c}=$ $x^{2} \tau_{m}$. Note that we have used small $\mathrm{x}$ approximation, which gives a cutoff frequency $\omega_{c}=\frac{1}{x^{2} \tau_{m}}$. The corresponding time scale is just $\tau_{c}$, which means that we can not probe the property of neurons occurred smaller than $\tau_{c}$ under the linear approximation. So we will take $\sigma_{\dot{V} x}^{2}=C_{\dot{V} x}\left(\tau_{c}\right)$, while for $\sigma_{V x}^{2}$ we still take $\sigma_{V x}^{2}=C_{V x}(0)$,

$$
\begin{aligned}
\sigma_{V x}^{2} & =C_{V x}(0) \\
& \approx \sigma_{V}^{2}\left(1-\frac{2 x}{\tau_{D}-\tau_{R}}\left(\tau_{D}\left(1+\frac{\tau_{m}}{\tau_{D}}\right)^{\frac{1}{2}}-\tau_{R}\left(1+\frac{\tau_{m}}{\tau_{R}}\right)^{\frac{1}{2}}\right)\right) \\
\sigma_{\dot{V} x}^{2} & =C_{\dot{V} x}\left(\tau_{c}\right) \\
& \left.\approx \frac{\sigma_{V}^{2}}{\tau_{D} \tau_{R}}\left(1-\frac{2 x}{\tau_{D}-\tau_{R}}\left(\tau_{R}\left(1+\frac{\tau_{m}}{\tau_{D}}\right)^{\frac{1}{2}}-\tau_{D}\left(1+\frac{\tau_{m}}{\tau_{R}}\right)^{\frac{1}{2}}\right)\right)+3 x^{2} \frac{\tau_{D}+\tau_{R}}{\tau_{D} \tau_{R}} \tau_{m}\right) .
\end{aligned}
$$

In the simple ball-and-stick model, the linear response $\nu_{1 x}$ is given by

$$
\begin{aligned}
\nu_{1 x}(\omega) & =\frac{1}{2 \pi \sigma_{V x}} e^{-\frac{\psi_{0}^{2}}{2 \sigma_{V x}^{2}}\left(\frac{\sigma_{\dot{V} x} \psi_{0}}{\sigma_{V x}^{2}}+\sqrt{\frac{\pi}{2}} i \omega\right) \frac{1}{\sqrt{1+i \tau_{m} \omega}\left(\sqrt{1+i \tau_{m} \omega}+K\right)} e^{-x \sqrt{1+i \tau_{m} \omega}}} \\
& =\nu_{0 x} \frac{\psi_{0}}{\sigma_{V x}^{2}} \frac{1+i \tau_{e f f} \omega}{\left(\sqrt{1+i \tau_{m} \omega}+K\right) \sqrt{1+i \tau_{m} \omega}} e^{-x \sqrt{1+i \tau_{m} \omega}}
\end{aligned}
$$

where

$$
\tau_{e f f}=\sqrt{\frac{\pi}{2}} \frac{\sigma_{V x}^{2}}{\psi_{0} \sigma_{\dot{V} x}}
$$

is an effective time constant. For low frequency the phase shift will develop a minimum at $\omega=1 / \sqrt{\tau_{e f f} \tau_{m}^{\prime}}$ with $\tau_{m}^{\prime}=\frac{K+2}{2(K+1)} \tau_{m}$ and the minimum is approximately given by $\frac{1}{2(K+1)} \frac{\tau_{m}^{\prime}-\tau_{e f f}}{\sqrt{\tau_{m}^{\prime} \tau_{e f f}}}$. For large $\omega$,there are the following limit behavior,

$$
\begin{aligned}
\left|\nu_{1 x}\right| & =\nu_{0 x} \frac{\psi_{0}}{\sigma_{V x}^{2}} \frac{\tau_{\text {eff }}}{\tau_{m}} e^{-x \sqrt{\tau_{m} \omega / 2}}, \\
\phi & =-x \sqrt{\tau_{m} \omega / 2} .
\end{aligned}
$$

\section{Results in coupled point Gaussian neurons}

The dynamics of two coupled Gaussian point neurons is defined by

$$
\begin{aligned}
& \tau_{1} \dot{V}_{1}=-V_{1}+c\left(V_{2}-V_{1}\right), \\
& \tau_{2} \dot{V}_{2}=-V_{2}+c\left(V_{1}-V_{2}\right)+\mu,
\end{aligned}
$$


where $\tau_{1}, V_{1}(t)$ and $\tau_{2}, V_{2}(t)$ are the membrane time constant and membrane potential of neuron 1 and neuron 2 (or compartment 1 and compartment 2) respectively. $\mu(t)$ is the injected current to neuron 2. $c$ is the coupling strength between the two neurons. This two coupled neurons provide a simplified system for the two-compartment homogeneous cable. We will derive the Green's function corresponding to a current injected in neuron 2, which tells the response at neuron 1 to a $\delta$ pulse input. Spikes is assumed to be triggered at neuron 1 . The correlation function of neuron 2 is assumed to have the form given in Eq. (5.38). We will calculate the linear response at neuron 1.

Eq. (5.75) can be solved by performing the Laplace transform,

$$
\begin{aligned}
V_{1}(s) & =\frac{c}{1+c+\tau_{1} s} V_{2}(s) \\
& =\frac{c}{\left(1+c+\tau_{1} s\right)\left(1+c+\tau_{2} s\right)-c^{2}} \mu,
\end{aligned}
$$

According to Eq. (5.76), the correlation function of $V_{1}$ is given by

$$
\begin{aligned}
C_{V_{1}}(\tau) & =\left\langle V_{1}(t) V_{1}(t+\tau)\right\rangle \\
& =\frac{1}{2 \pi} \int_{-\infty}^{+\infty}\left|V_{1}(\omega)\right|^{2} e^{i \omega t} \\
& =\frac{1}{2 \pi} \int_{-\infty}^{+\infty} \frac{c^{2}}{(1+c)^{2}+\tau_{1}^{2} \omega^{2}} P_{V_{2}}(\omega) e^{i \omega t}
\end{aligned}
$$

where $P_{V_{2}}$ is the power spectrum of neuron 1, given by Eq. (5.41).

Performing the integral,

$$
\begin{aligned}
C_{V_{1}}(\tau)= & \frac{c^{2} \sigma_{V}^{2}}{\tau_{D}-\tau_{R}}\left(\frac{\tau_{1} \tau_{D}^{2}}{(1+c)\left(\tau_{1}^{2}-(1+c)^{2} \tau_{D}^{2}\right)} e^{-(1+c)|\tau| / \tau_{1}}+\right. \\
& \left.\frac{\tau_{D}^{3}}{(1+c)^{2}-\tau_{1}^{2}} e^{-|\tau| / \tau_{D}}-\left(\tau_{D} \leftrightarrow \tau_{R}\right)\right) \\
\sigma_{V_{1}}^{2}= & C_{V_{1}}(0)=\sigma_{V}^{2} \cdot \frac{c^{2}}{(1+c)^{2}} \cdot \frac{\tau_{D} \tau_{R}+\tau_{H}\left(\tau_{D}+\tau_{R}\right)}{\left(\tau_{D}+\tau_{H}\right)\left(\tau_{R}+\tau_{H}\right)},
\end{aligned}
$$

where $\tau_{H}=\tau_{1} /(1+c)$ and

$$
\sigma_{\dot{V}_{1}}^{2}=-C_{V_{2}}^{\prime \prime}(0)=\sigma_{V}^{2} \cdot \frac{c^{2}}{(1+c)^{2}} \cdot \frac{1}{\left(\tau_{D}+\tau_{H}\right)\left(\tau_{R}+\tau_{H}\right)} .
$$

The stationary firing rate $\nu_{0}$ of neuron 1 is

$$
\nu_{0}=\frac{\sigma_{\dot{V}_{1}}}{2 \pi \sigma_{V_{1}}} \exp \left(-\frac{\psi_{0}^{2}}{2 \sigma_{V_{1}}^{2}}\right)
$$


The effective time constant as defined in Eq. (5.12) is given by

$$
\begin{aligned}
\tau_{e f f} & =\sqrt{\frac{\pi}{2}} \frac{\sigma_{V_{1}}^{2}}{\sigma_{\dot{V}_{1}} \psi_{0}} \\
& =\sqrt{\frac{\pi}{2}} \frac{\sigma_{V}}{\psi_{0}} \frac{c}{1+c} \frac{\tau_{D} \tau_{R}+\tau_{H}\left(\tau_{D}+\tau_{R}\right)}{\sqrt{\left(\tau_{D}+\tau_{H}\right)\left(\tau_{R}+\tau_{H}\right)}} .
\end{aligned}
$$

When a small current $\varepsilon e^{i \omega t}$ is injected at soma, from Eq. (5.76) the filtered signal at position $\mathrm{x}$ is given by

$$
f_{1}(t)=\varepsilon \frac{c}{\left(1+c+i \omega \tau_{1}\right)\left(1+c+i \omega \tau_{2}\right)-c^{2}} e^{i \omega t},
$$

and therefore the linear response reads

$$
\begin{aligned}
\nu_{1}(\omega) & =c \nu_{0} \frac{\psi_{0}}{\sigma_{V_{1}}^{2}} \frac{1+i \omega \tau_{e f f}}{\left(1+c+i \omega \tau_{1}\right)\left(1+c+i \omega \tau_{2}\right)-c^{2}} \\
& =\nu_{0} \frac{\psi_{0}}{\sigma_{V}^{2}} \frac{(1+c)^{2}}{c} \frac{\left(\tau_{D}+\tau_{H}\right)\left(\tau_{R}+\tau_{H}\right)}{\tau_{D} \tau_{R}+\tau_{H}\left(\tau_{D}+\tau_{R}\right)} \frac{1+i \omega \tau_{e f f}}{\left(1+c+i \omega \tau_{1}\right)\left(1+c+i \omega \tau_{2}\right)-c^{2}}
\end{aligned}
$$

Note that when $c \rightarrow 0$, the neuron 1 will not be influenced by the injected current at neuron 2, and will take the resting potential. The stationary firing rate will also decay to zero exponentially, as seen from Eq. (5.81). Therefore at zero coupling the linear response will also be zero. When $c \rightarrow \infty$, the two neurons are synchronized, $V_{1}=V_{2}=\mu$, and the response is independent with $c$. 


\section{Chapter 6}

\section{Summary and Perspective}

In this thesis, the properties of signal encoding in cortical neurons were investigated theoretically through analytical solvable neuron models. Cortical neurons can encode noisy time-varying signals through population encoding. The dynamical response to fast changing signals was observed to have cutoff frequencies much higher than inverse membrane time constant and the average firing rate, which is unexpected from previous theoretical studies. We addressed this problem by constructing analytically tractable spiking neuron models, which include the spike initiation process explicitly. This new kind of models has piecewise-linear dynamics with each piece having clear physical meaning and providing analytical tractability. We showed that the artifactual behavior that results from the truncation of the AP waveform can be mathematically isolated, resulting to a biophysically meaningful response function. Since cortical neurons are spatially extended and APs are generated in the proximal axonal segment, we also investigated the AP encoding properties in spatially extended neurons assuming the membrane potential as a Gaussian random field.

In Chapter 2, a simple spiking neuron model with two linear pieces in its dynamics was constructed to investigate the relation between the spike onset rapidness of individual neurons and the cutoff frequencies of the dynamical response in a population of neurons by assuming the synaptic noise to be a Gaussian white noise. We found that when the external signal is encoded in the variance of synaptic noise, the cutoff frequency is directly scaled with the onset rapidness of individual neurons. But for a signal encoded in the mean input current, the cutoff frequency is confined by the membrane time constant. Since in cortical neurons the spike onset was observed to be very fast, we thus reached a quantitative understanding for the high cutoff frequency for the noise coded signal. For mean coded signals other factors apparently need to be taking into account.

In chapter 3 we more specially studied one mechanism that leads to a large AP onset rapidness and considered the effect of a finite correlation time in the synaptic noise. Cooperative opening of sodium channels has been proposed to explain the fast onset observed in neurophysiological experiments. A directive 
consequence of this cooperativity is a step change in the open probability of sodium channels, which can result in a step change in the spike generating current. We incorporated this step change into our models and studied its role in the linear response to a time-varying signal. We found that a step change in the spike generating current can enhance the cutoff frequency and corresponds to an effective increase of the onset rapidness. In the large step change limit, the model was proved to reduce to the LIF model at the linear order. By taking into account the temporal correlations in the synaptic noise, we found that the cutoff frequency can be significantly enhanced towards high frequency especially when the onset rapidness is large, and the confinement by the membrane time constant for a current coded signal is broken. When a step change was included, significant enhancement on the cutoff frequency in the colored noise case was observed even for a small step size, although the effect is negligible for white noise. It was previously found that in the LIF model the response function for a mean coded signal does not attenuate at high cutoff frequency when the correlation time in the synaptic noise is included. Our results thus showed that for current coded signals the high cut-off frequency phenomenon can be understood if the AP onset rapidness is large (including the case for a step change in the spike generating current) and the correlation time in the synaptic noise is finite.

Thus our conclusions from this chapter are that if the AP onset rapidness is very large, then the high cutoff frequency phenomenon can be understood by its own, since real synaptic correlation is finite ranging from several milliseconds to hundreds of milliseconds. If the onset rapidness is not so large, then the cooperativity induced step change in the spike generating current may explain the high cutoff frequency well. Although experiments suggest that the spike onset is fast, what is measured in experiments is the slope in the phase plot at some $\dot{V}$, say $10 \mathrm{mV} / \mathrm{ms}$. It corresponds to a slope at the exponential spike generating curve at that point, but does not give directly the effective AP onset rapidness. In our work we define an effective time constant to describe the spike initiation process. We approximated the spike initiation process using an effective linear current with its slope measuring the ratio between the membrane time constant and the effective spike initiation time constant and defined as the AP onset rapidness.

In chapter 4 we constructed a general piecewise linear model which includes the previously constructed models as limiting cases and are still analytically solvable. This model describes the subthreshold dynamics and spike initiation by two separate parts and therefore can be used to study the influence of different parts of the spike initiation process on the dynamical response. We find that the subthreshold part mainly influences the stationary response and has little influence on the dynamical response. We further construct a 3-piece model which has the same rheobase current and spike initiation threshold as the EIF model, while the spike onset rapidness can be obtained by fitting the $f-I$ curve. The resulted 3 -piece model reproduces the $f-I$ curve very well and in addition provides good 
agreement for the linear response. The fitted onset rapidness for cortical neurons is 100, while for hippocortical interneurons, the resulted onset rapidness is 10 . The large onset rapidness for cortical neurons could easily give cutoff frequencies at hundreds $\mathrm{Hz}$ for both fast and slow synaptic correlations. It is thus very plausible to propose that the high onset rapidness underlies the surprising recent experimental observation of high cut-off frequencies in cortical neurons.

In Chapter 5, we studied signal encoding in spatially extended neurons and the functional consequence of the AIS initiation of APs. The axon was modeled as a homogeneous cable with two compartments describing the AIS and the other part of axon. The soma was taken as a point at the end of the cable with a dynamics taking into account the leak current. By combing the linear cable equation and the Gaussian random field assumption we calculated the correlation functions along the axon and the spike-triggered average at soma when spikes are triggered at the AIS. The spike-triggered variance accounted for only a small fraction of the variability of membrane potential observed in experiments. We calculated the linear response analytically when an oscillating signal is injected into the soma and found that a small time constant at the AIS can enhance the high frequency response significantly and increase the cutoff frequencies. Thus an additional specialization of the AIS membrane may further augment high frequency encoding in cortical neurons.

In one-variable spiking neuron models, an absorbing boundary is usually required and the membrane potential is reset there to some subthreshold value to restart the temporal evolution. An absorbing boundary at a finite value of membrane potential has been shown to produce severely artifactual behavior in the dynamical response, e.g., a transmission function without attenuation at high signal frequency. In the piecewise linear models that we have constructed in this thesis, the linear response can be solved analytically and the artifacts induced by the absorbing boundary can be separated out mathematically. The resulting biophysically relevant part depends on the absorbing boundary only through a frequency dependent phase lag, which reflects the soliton behavior of APs. The resulting response function decays for high frequencies as a combination of power law and exponential decay. One should note that in the nonlinear IF neuron models the absorbing boundary can be sent to infinity, which makes the transmission function decay in general as power law. This decaying behavior, however, is determined by the ratio between the spike generating current and its derivatives at the limit $v \rightarrow \infty$, i.e., by the dynamics at the position of the (infinite) boundary. Whether an infinite threshold introduces some artifacts in the dynamical response calls for further mathematical investigation.

The dynamical response of a nonlinear system is controlled by its intrinsic time constants. In neuron system, the membrane time constant is a slow time constant compared with the fast AP onset process which plays an important role in shaping the dynamical response. We defined an effective fast time constant characterizing the AP onset process by introducing a linear spike generating current. The AP 
onset rapidness is defined as the ratio between the slow and the fast time constant. We provided a way to extract the AP onset rapidness for a type I neuron by fitting a piecewise linear model to an EIF model. In this way we show clearly that the cortical neurons has a very large AP onset rapidness which allows for high cutoff frequencies in the linear response.

In summary, the works in this thesis provide a novel theoretical foundation and machinery to study signal encoding in the neuronal population through analytically tractable spiking neuron models which include the AP initiation process explicitly. Obviously there are some future direction and extension of the present work. First, the analytical study of the high frequency behavior in the colored noise case. This should provide a definite relation that governs the interaction of the external and intrinsic time constants: the membrane time constant $\tau_{m}$, the effective time constant for the spike initiation process and the correlation time of the synaptic noise. The functional effect of AIS in signal encoding also could profit from further investigation using nonlinear multi-compartment models. It is hoped that the exact and analytical results presented in this thesis will provide rational guidance and help to quantitatively control such investigations. 


\section{Bibliography}

Abramowitz, M. \& Stegun, I. A. (1970). Handbook of Mathematical functions. (New York: Dover).

Attwell, D. \& Laughlin, S. B. (2001). An energy budget for signaling in the grey matter of the brain. J Cereb Blood Flow Metab, 21, 1133-45.

Badel, L., Lefort, S., Brette, R., Petersen, C. C. H., Gerstner, W., \& Richardson, M. J. E. (2008). Dynamic I-V curves are reliable predictors of naturalistic pyramidal-neuron voltage traces. J Neurophysiol, 99, 656-66.

Baranauskas, G., Mukovskiy, A., Wolf, F., \& Volgushev, M. (2010). The determinants of the onset dynamics of action potentials in a computational model. Neuroscience, 167, 1070-90.

Boucsein, C., Tetzlaff, T., Meier, R., Aertsen, A., \& Naundorf, B. (2009). Dynamical response properties of neocortical neuron ensembles: multiplicative versus additive noise. J Neurosci, 29, 1006-10.

Bray, D. \& Duke, T. (2004). Conformational spread: the propagation of allosteric states in large multiprotein complexes. Annu Rev Biophys Biomol Struct, 33, $53-73$.

Brunel, N., Chance, F. S., Fourcaud, N., \& Abbott, L. F. (2001). Effects of synaptic noise and filtering on the frequency response of spiking neurons. Phys Rev Lett, 86, 2186-2189.

Bulsara, A. R., Lowen, S. B., \& Rees, C. D. (1994). Cooperative behavior in the periodically modulated wiener process: Noise-induced complexity in a model neutron. Phys. Rev. E, 49, 4989-5000.

Burak, Y., Lewallen, S., \& Sompolinsky, H. (2009). Stimulus-dependent correlations in threshold-crossing spiking neurons. Neural Comput, 21, 2269-308.

Burns, B. D. \& Webb, A. C. (1976). The spontaneous activity of neurones in the cat's cerebral cortex. Proc R Soc Lond B Biol Sci, 194, 211-23. 
Campbell, G. A. \& Foster, R. M. (1961). Fourier integrals for practical applications. (Princeton, N.J: D. Van Nostrand).

Chow, C. C. \& White, J. A. (1996). Spontaneous action potentials due to channel fluctuations. Biophys J, 71, 3013-21.

Clark, B. D., Goldberg, E. M., \& Rudy, B. (2009). Electrogenic tuning of the axon initial segment. Neuroscientist, 15, 651-68.

Destexhe, A. \& Paré, D. (1999). Impact of network activity on the integrative properties of neocortical pyramidal neurons in vivo. J Neurophysiol, 81, 153147.

DeWeese, M. R., Wehr, M., \& Zador, A. M. (2003). Binary spiking in auditory cortex. J Neurosci, 23, 7940-9.

Ermentrout, B. (1996). Type I membranes, phase resetting curves, and synchrony. Neural Comput, 8, 979-1001.

Fleidervish, I. A., Lasser-Ross, N., Gutnick, M. J., \& Ross, W. N. (2010). $\mathrm{Na}^{+}$ imaging reveals little difference in action potential-evoked $\mathrm{Na}^{+}$influx between axon and soma. Nat Neurosci, 13, 852-60.

Fourcaud, N. \& Brunel, N. (2002). Dynamics of the firing probability of noisy integrate-and-fire neurons. Neural Comput, 14, 2057-110.

Fourcaud-Trocme, N. \& Brunel, N. (2005). Dynamics of the instantaneous firing rate in response to changes in input statistics. J Comput Neurosci, 18, 311-21.

Fourcaud-Trocme, N., Hansel, D., van Vreeswijk, C., \& Brunel, N. (2003). How spike generation mechanisms determine the neuronal response to fluctuating inputs. J Neurosci, 23, 11628-40.

Fusi, S. \& Mattia, M. (1999). Collective behavior of networks with linear (VLSI) integrate-and-fire neurons. Neural Comput, 11, 633-52.

Geisler, C., Brunel, N., \& Wang, X.-J. (2005). Contributions of intrinsic membrane dynamics to fast network oscillations with irregular neuronal discharges. J Neurophysiol, 94, 4344-61.

Gerstein, G. L. \& Mandelbrot, B. (1964). Random walk models for the spike activity of a single neuron. Biophys J, 4, 41-68.

Gerstner, W. (2000). Population dynamics of spiking neurons: fast transients, asynchronous states, and locking. Neural Comput, 12, 43-89.

Grubb, M. S. \& Burrone, J. (2010). Activity-dependent relocation of the axon initial segment fine-tunes neuronal excitability. Nature, 465, 1070-4. 
Hedstrom, K. L. \& Rasband, M. N. (2006). Intrinsic and extrinsic determinants of ion channel localization in neurons. J Neurochem, 98, 1345-1352.

Higgs, M. H. \& Spain, W. J. (2009). Conditional bursting enhances resonant firing in neocortical layer 2-3 pyramidal neurons. J Neurosci, 29, 1285-99.

Hodgkin, A. L. \& Huxley, A. F. (1952). A quantitative description of membrane current and its application to conduction and excitation in nerve. J Physiol, $117,500-544$.

Hodgkin, A. L. \& Rushton, W. A. H. (1946). The electrical constants of a crustacean nerve fibre. Proc R Soc Med, 134, 444-79.

Honeycutt, R. L. (1992a). Stochastic runge-kutta algorithms. i. white noise. Phys. Rev. A, 45, 600-603.

Honeycutt, R. L. (1992b). Stochastic runge-kutta algorithms. ii. colored noise. Phys. Rev. A, 45, 604-610.

Howard, A., Tamas, G., \& Soltesz, I. (2005). Lighting the chandelier: new vistas for axo-axonic cells. Trends Neurosci, 28, 310-6.

Huang, M. (2009). Spatial-temporal dynamics of patten formation in the cerebral cortex. Ph.D. thesis, University of Göttingen, Germany.

Inda, M. C., DeFelipe, J., \& Munoz, A. (2006). Voltage-gated ion channels in the axon initial segment of human cortical pyramidal cells and their relationship with chandelier cells. Proc Natl Acad Sci USA, 103, 2920-2925.

Jung, P. (1994). Threshold devices: Fractal noise and neural talk. Phys. Rev. E, $50,2513-2522$.

Knight, B. W. (1972a). Dynamics of encoding in a population of neurons. J Gen Physiol, 59, 734-766.

Knight, B. W. (1972b). The relationship between the firing rate of a single neuron and the level of activity in a population of neurons. experimental evidence for resonant enhancement in the population response. J Gen Physiol, 59, 767-778.

Kole, M. H., Ilschner, S. U., Kampa, B. M., Williams, S. R., Ruben, P. C., \& Stuart, G. J. (2008). Action potential generation requires a high sodium channel density in the axon initial segment. Nat Neurosci, 11, 178-186.

Kole, M. H. P. \& Stuart, G. J. (2008). Is action potential threshold lowest in the axon? Nat Neurosci, 11, 1253-5. 
Köndgen, H., Geisler, C., Fusi, S., Wang, X. J., Lüscher, H. R., \& Giugliano, M. (2008). The dynamical response properties of neocortical neurons to temporally modulated noisy inputs in vitro. Cereb Cortex, 18, 2086-97.

Kuba, H., Ishii, T. M., \& Ohmori, H. (2006). Axonal site of spike initiation enhances auditory coincidence detection. Nature, 444, 1069-72.

Kuba, H., Oichi, Y., \& Ohmori, H. (2010). Presynaptic activity regulates $\mathrm{Na}^{+}$ channel distribution at the axon initial segment. Nature, 465, 1075-8.

Lapicque, L. (1907). Recherches quantitatives sur l'excitation électrique des nerfs traitée comme une polarisation. J. Physiol. (Paris), 9, 620-635.

Laughlin, S. B. \& Sejnowski, T. J. (2003). Communication in neuronal networks. Science, 301, 1870-4.

Lennie, P. (2003). The cost of cortical computation. Curr Biol, 13, 493-7.

Lindner, B. \& Schimansky-Geier, L. (2001). Transmission of noise coded versus additive signals through a neuronal ensemble. Phys. Rev. Lett., 86, 2934-2937.

Liu, Y. \& Dilger, J. P. (1993). Application of the one- and two-dimensional Ising models to studies of cooperativity between ion channels. Biophys J, 64, 26-35.

Longtin, A. (1993). Stochastic reasonance in neuron models. J Stat Phys, 70, $309-27$.

Margrie, T. W., Brecht, M., \& Sakmann, B. (2002). In vivo, low-resistance, wholecell recordings from neurons in the anaesthetized and awake mammalian brain. Pflugers Arch, 444, 491-8.

Marx, S. O., Ondrias, K., \& Marks, A. R. (1998). Coupled gating between individual skeletal muscle $\mathrm{Ca}^{2+}$ release channels (ryanodine receptors). Science, $281,818-821$.

McCormick, D. A., Shu, Y., \& Yu, Y. (2007). Neurophysiology: Hodgkin and huxley model-still standing? Nature, 445, 1-2.

Meeks, J. P. \& Mennerick, S. (2007). Action potential initiation and propagation in ca3 pyramidal axons. J Neurophysiol, 97, 3460-3472.

Milshtein, G. N. \& Tret'yakov, M. V. (1994). Numerical solution of differential equations with colored noise. J. Stat. Phys., 77, 691-715.

Molina, M., Barrera, F., Fernandez, A., Poveda, J., Renart, M., Encinar, J., Riquelme, G., \& Gonzalez-Ros, J. M. (2006). Clustering and coupled gating modulate the activity in KcsA, a potassium channel model. J. Biol. Chem., $281,18837-18848$. 
Naundorf, B. (2005). Dynamics of population coding in the cortex. Ph.D. thesis, University of Göttingen, Germany.

Naundorf, B., Geisel, T., \& Wolf, F. (2005). Action potential onset dynamics and the response speed of neuronal populations. J. Comput. Neurosci., 18, 297-309.

Naundorf, B., Wolf, F., \& Volgushev, M. (2006). Unique features of action potential initiation in cortical neurons. Nature, 440, 1060-3.

Naundorf, B., Wolf, F., \& Volgushev, M. (2007). Neurophysiology: Hodgkin and huxley model - still standing? (reply). Nature, 445, E2-E3.

Palmer, L. M. \& Stuart, G. J. (2006). Site of action potential initiation in layer 5 pyramidal neurons. J Neurosci, 26, 1854-1863.

Plesser, H. E. \& Geisel, T. (1999). Markov analysis of stochastic resonance in a periodically driven integrate-and-fire neuron. Phys. Rev. E, 59, 7008-7017.

Populin, L. C. \& Yin, T. C. (1998). Behavioral studies of sound localization in the cat. J Neurosci, 18, 2147-60.

Pressley, J. \& Troyer, T. W. (2009). Complementary responses to mean and variance modulations in the perfect integrate-and-fire model. Biol Cybern, 101, 63-70.

Rall, W. (1959). Branching dendritic trees and motoneuron membrane resistivity. Exp Neurol, 1, 491-527.

Rall, W. (1977). Core conductor theory and cable properties of neurons. In Kandel, E.R., J.M. Brookhardt, and V.M. Mountcastle eds. Handbook of physiology, cellular biology of neurons. (Bethesda, MD: American Physiology Society).

Risken, H. (1984). The Fokker-Planck equation: Methods of solution and applications. (Springer Verlag).

Saito, A., Inui, M., Radermacher, M., Frank, J., \& Fleischer, S. (1988). Ultrastructure of the calcium release channel of sarcoplasmic reticulum. J Cell Biol, $107,211-219$.

Shadlen, M. N. \& Newsome, W. T. (1998). The variable discharge of cortical neurons: implications for connectivity, computation, and information coding. J Neurosci, 18, 3870-96.

Shu, Y., Hasenstaub, A., Duque, A., Yu, Y., \& McCormick, D. A. (2006). Modulation of intracortical synaptic potentials by presynaptic somatic membrane potential. Nature, 441, 761-5. 
Silberberg, G., Bethge, M., Markram, H., Pawelzik, K., \& Tsodyks, M. (2004). Dynamics of population rate codes in ensembles of neocortical neurons. J Neurophysiol, 91, 704-9.

Softky, W. R. \& Koch, C. (1993). The highly irregular firing of cortical cells is inconsistent with temporal integration of random EPSPs. J Neurosci, 13, $334-50$.

Somogyi, P., Freund, T., \& Cowey, A. (1982). The axo-axonic interneuron in the cerebral cortex of the rat, cat and monkey. Neuroscience, 7, 2577-607.

Stanford, T. R., Shankar, S., Massoglia, D. P., Costello, M. G., \& Salinas, E. (2010). Perceptual decision making in less than 30 milliseconds. Nat Neurosci, $13,379-85$.

Stuart, G., Spruston, N., Sakmann, B., \& Häusser, M. (1997). Action potential initiation and backpropagation in neurons of the mammalian cns. Trends Neurosci, 20, 125-131.

Tchumatchenko, T., Malyshev, A., Geisel, T., Volgushev, M., \& Wolf, F. (2010). Correlations and synchrony in threshold neuron models. Phys Rev Lett, 104, 058102 .

Tuckwell, H. C. (1988). Introduction totheoretical neurobiology, Volume I and II. (Cambridge: Cambridge University Press).

Tuckwell, H. C. \& Walsh, J. B. (1983). Random currents through nerve membranes. I. Uniform poisson or white noise current in one-dimensional cables. Biol Cybern, 49, 99-110.

Tuckwell, H. C., Wan, F. Y., \& Wong, Y. S. (1984). The interspike interval of a cable model neuron with white noise input. Biol Cybern, 49, 155-67.

Tuckwell, H. C., Wan, F. Y. M., \& Rospars, J.-P. (2002). A spatial stochastic neuronal model with Ornstein-Uhlenbeck input current. Biol Cybern, 86, 13745 .

Undrovinas, A., Fleidervish, I., \& Makielski, J. (1992). Inward sodium current at resting potentials in single cardiac myocytes induced by the ischemic metabolite lysophosphatidylcholine. Circ. Res., 71, 1231-1241.

VanRullen, R. \& Thorpe, S. J. (2001). Is it a bird? Is it a plane? Ultra-rapid visual categorisation of natural and artifactual objects. Perception, 30, 655-68.

Wan, F. Y. \& Tuckwell, H. C. (1979). The response of a spatially distributed neuron to white noise current injection. Biol Cybern, 33, 39-55. 
Wang, X. J. \& Buzsáki, G. (1996). Gamma oscillation by synaptic inhibition in a hippocampal interneuronal network model. J Neurosci, 16, 6402-13.

Wolf, F. \& Geisel, T. (1998). Spontaneous pinwheel annihilation during visual development. Nature, 395, 73-8.

Yu, Y., Shu, Y., \& McCormick, D. A. (2008). Cortical action potential backpropagation explains spike threshold variability and rapid-onset kinetics. J Neurosci, 28, 7260-7272. 


\section{Acknowledgements}

First I would like to thank my advisor Prof. Fred Wolf for leading me into the fascinating field of theoretical neuroscience. His enthusiasm and deep insight for science have guided me through the thesis research and will continue to be my source of inspiration and courage.

Thanks to Prof. Tobias Moser and Prof. Eberhard Bodenschatz for being my thesis committee members and for their suggestions and discussions on the research project. Thanks to Prof. Kree Reiner for being the second referee of my thesis and Prof. Marc Timme and Dr. Alexander Gail for being in my examination board.

Thanks to Prof. Theo Geisel for heading the vigorous department and making the wonderful dynamical symposium possible. Thanks to Prof. Michael Gutnick and Prof. Ilya Fleidervish for the warmly hospitality which makes the staying in Israel a memorial experience and for the discussion on my work. Thanks to Prof. Benjamin Lindner, Prof. Elisha Moses, Dr. Demian Battaglia and Dr. Tzvetomir Tzvetanov for suggestions and comments on my work.

Thanks to my colleagues Min, Tatjana, Mike, Pinar, Georg, Demian, Andreas, Raoul, Wolfgang, Anna, Michael, Lars, Lishma, Nikolai, and many others for the discussion and warm help in scientific and non-scientific problems.

Thanks to Corrinna Trautsch, Kathraina Jeremias, Tanja Gindela, Regina Wunderlich and Dr. Tobias Niemann for administrative support. Thanks to York-Fabian Beensen and Georg Martius for the kind help related to computers.

Thanks to Min, Tzvetomir, Jin for spending so much wonderful time together. Thanks to Angelina and Christa for the kind help.

Finally, I would like to thank my parents for the support in the past years. Thanks to my wife Xuejie, for giving a new meaning to my life and unlimited supporting. 


\section{Curriculum Vitae}

Forename: Wei $\quad$ Wei

Surname: Wei

Date of Birth: Jun. 08, 1979

Place of Birth : Liaoning, P. R. China

Citizenship: China
Address: Am Fassberg. 17

37077, Göttingen

Phone: $\quad+49(0) 17664818884$

Email: $\quad$ wei@nld.ds.mpg.de

\section{Education}

2007-2011 PhD study in Neurosciences,

Max-Planck-Institute for Dynamics \& Self-organization, Göttingen Advisor: Prof. Fred Wolf

2002-2007 Ph.D in Theoretical Physics,

Peking University

Advisors: Prof. Da-Hai Lu and Prof. Shi-Lin Zhu

1998-2002 B.E in Aeronautic Engineering,

Shenyang Institute of Aeronautic Technology

\section{Teaching Experience}

2008-2009 Teaching assistant for Mathematical Physics II,

Department of Physics, Göttingen University

2004-2005 Teaching assistant for Quantum Mechanics (A and B),

Department of Physics, Peking University 


\section{Publications}

\section{On High Energy Physics}

1. W. Wei, X. Liu, and Shi-Lin Zhu, D wave Heavy Mesons, Phys. Rev. D 75 (2007) 014013.

2. W. Wei, P-Z Huang and Shi-Lin Zhu, Strong decays of $D_{s J}(2317)$ and $D_{s J}(2460)$, Phys. Rev. D 73 (2006) 034004.

3. W. Wei, L. Zhang and Shi-Lin Zhu, The possible $J^{P C} I^{G}=2^{++} 2^{+}$state X(1600), Int.J.Mod.Phys. A21 (2006) 4617.

4. W. Wei, P-Z. Huang, H-X Chen and Shi-Lin Zhu, Spin $\frac{3}{2}$ pentaquarks, JHEP 0507 (2005) 015.

\section{On Neuroscience}

1. W. Wei and F. Wolf, Spike Onset Dynamics and Response Speed in Neuronal Populations, Phys. Rev. Lett. 106 (2011) 088102.

2. W. Wei and F. Wolf, The bandwidth of temporal population coding is set by the dynamics of action potential initiation, in preparation.

3. W. Wei and F. Wolf, An analytical tractable model for dynamic action potential encoding in spatially extended neurons, in preparation.

4. X-J. Chen, W. Wei, F. Wolf and D. Battaglia, Interacting subcircuits in a primary visual cortex column, in preparation 